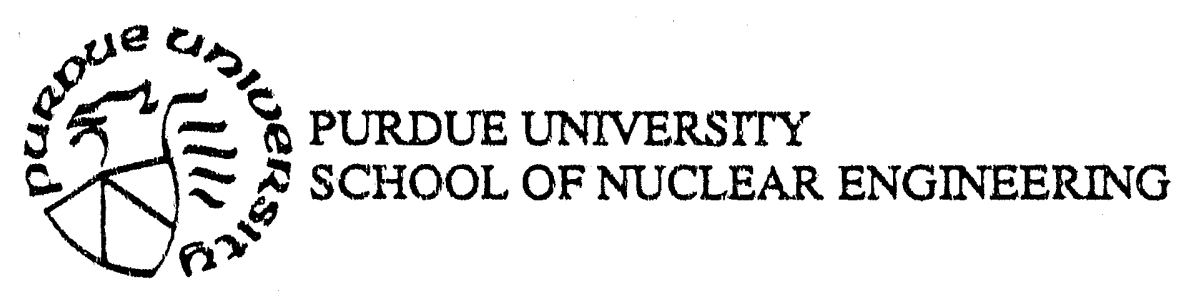

INTERFACIAL, AREA AND TWO-PHASE FLOW STRUCTURE DEVELOPMENT MEASURED BY A DOUBLE-SENSOR PROBE

by

Waihung Leung, Shripad T. Revankar Yoshihiko Ishii*, and Mamoru Ishii Thermalhydraulic and Reactor Safety Laboratory

June 1992

Work suppported by

U.S. DEPARTMENT OF ENERGY Office of Basic Energy Science

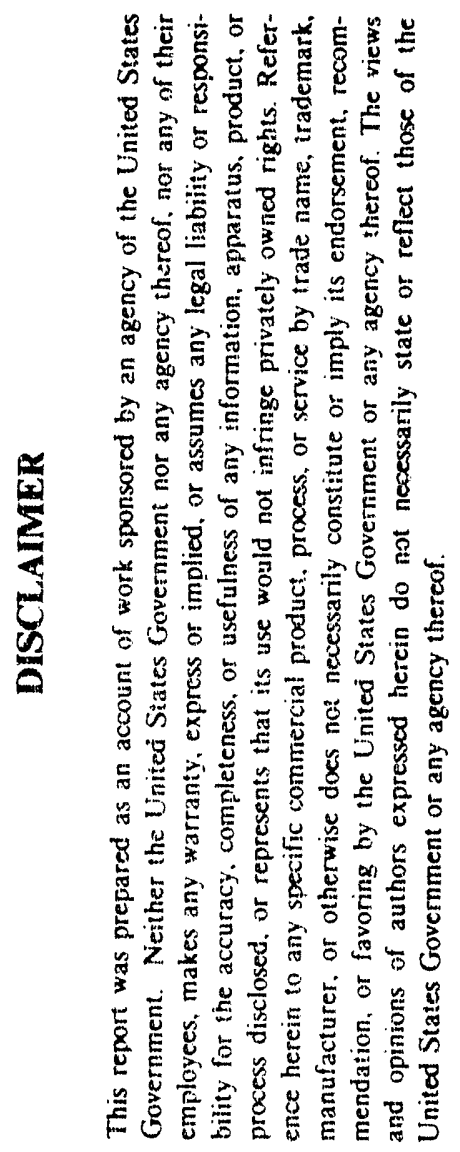




\section{TABLE OF CONTENTS}

PAGE

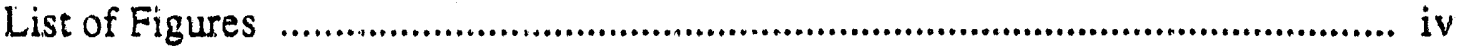

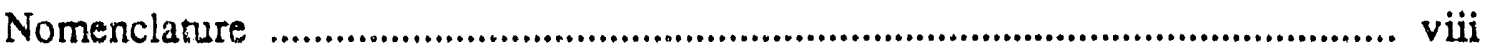

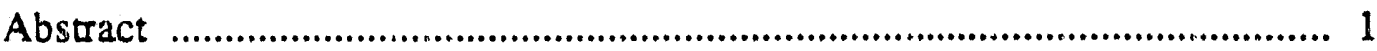

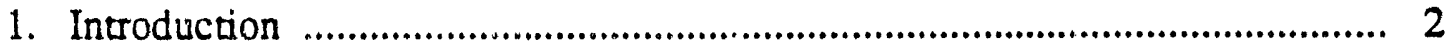

2. The Local Interfacial Area Concentration Measurement ............................... 6

3. The Local Measurement Method .............................................................. 15

4. Local Instrumentation

4.1 Double-Sensor Probe .................................................................. 20

4.2. Probe Design and Biasing ...................................................... 20

4.3 Signal Processing .................................................................. 22

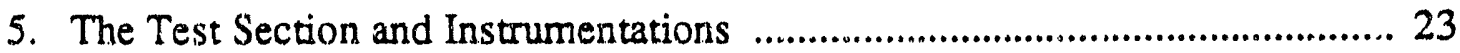

5.1 The Test Section ............................................................................. 18

5.2 Flow Rate Measurements

5.2.1 Superficial Gas Velocity .................................................... 26

5.2 .2 Superficial Liquid Velocity ................................................ 27

6. Experiment Results and Remarks

6.1 The Experiments ....................................................................... 27

6.2 Local Data Presentations .............................................................. 28

6.3 Observations

6.3.1 Constant Liquid Velocity .................................................... 30

6.3.2 Constant Gas Velocity ......................................................... 55

6.3.3 Comparison Plots ............................................................... 68

6.4 The Average Characters

6.4.1 The Drift Flux Model ................................................................. 110

6.4.2 Comparison of Average Values ............................................... 114

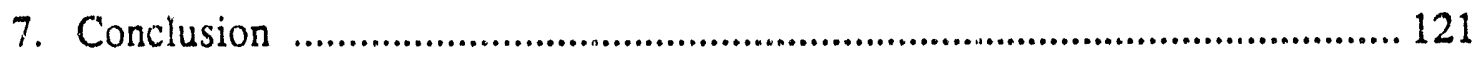

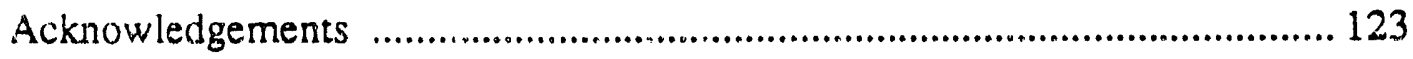

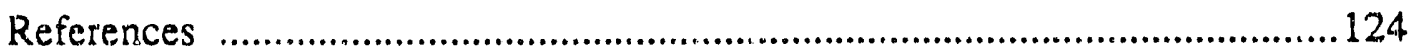




\section{LIST OF FIGURES}

No.

TITTLE

PAGE

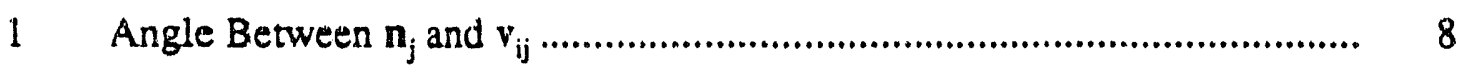

2 The Co-Ordinate and Angles of the Velocity Vector .............................. 10

3 The Co-Ordinate and Angles of the jth Interface Unit Normal ................ 10

$4 \quad$ Double-Sensor Probe and jth Incerface ............................................ $\quad 12$

5 The Probe Signals of a Train of Bubbles pass through the Sensors ............... 16

6 Double Sensor Probe Design Schematic ................................................. 19

$7 \quad$ The Sensor Design Schematic ............................................................ 19

8 The Equivalent Circuit of the Resistivity Probe ...................................... 21

9 The Trace of the Probe Signals ......................................................... 21

10 The Air-Water Loop Schematic ......................................................... 24

11 The Bubble Generator Design Schematic ........................................... 25

12 The Comparison of Average Void Fraction Measurement by Pressure

Drop and by Double-Sensor Probe (a) $L / D=8$, (b) $L / D=60$................... 29

13 The Radial Profile Plots For $j_{1}=1.0 \mathrm{~m} / \mathrm{s}$ and $L / D=8$,

(a) Void Fraction, (b) Interfacial Area Concentration, (c) Bubble Velocity,

(d) Sauter Mean Diameter, and (e) Bubble Frequency

14 The Radial Profile Plots For $j_{1}=1.0 \mathrm{~m} / \mathrm{s}$ and $\mathrm{L} / \mathrm{D}=60$,

(a) Void Fraction, (b) Interfacial Area Concentration, (c) Bubble Velocity,

(d) Sauter Mean Diameter, and (e) Bubble Frequency

15 The Radial Profile Plots For $j_{1}=0.6 \mathrm{~m} / \mathrm{s}$ and $L / D=8$,

(a) Void Fraction, (b) Interfacial Area Concentration, (c) Bubble Velocity,

(d) Sauter Mean Diameter, and (e) Bubble Frequency

16 The Radial Profile Plots For $j_{1}=0.6 \mathrm{~m} / \mathrm{s}$ and $\mathrm{L} / \mathrm{D}=60$,

(a) Void Fraction, (b) Interfacial Area Concentration, (c) Bubble Velocity,

(d) Sauter Mean Diameter, and (e) Bubble Frequency 
17. The Radial Profile Plots For $\mathrm{j}_{1}=0.4 \mathrm{~m} / \mathrm{s}$ and $\mathrm{L} / \mathrm{D}=8$,
(a) Void Fraction, (b) Interfacial Area Concentration, (c) Bubble Velocity,

(d) Sauter Mean Diameter, and (e) Bubble Frequency

18 The Radial Profile Plots For $j_{1}=0.4 \mathrm{~m} / \mathrm{s}$ and $\mathrm{L} / \mathrm{D}=60$,

(a) Void Fraction, (b) Interfacial Area Concentration, (c) Bubble Velocity,

(d) Sauter Mean Diameter, and (e) Bubbie Frequency

19 The Radial Profile Plots For $j_{1}=0.1 \mathrm{~m} / \mathrm{s}$ and $\mathrm{L} / \mathrm{D}=8$,

(a) Void Fraction, (b) Interfacial Area Concentration, (c) Bubble Velocity,

(d) Sauter Mean Diameter, and (e) Bubble Frequency

20 The Radial Profile Plots For $j_{1}=0.1 \mathrm{~m} / \mathrm{s}$ and $\mathrm{L} / \mathrm{D}=60$,

(a) Void Fraction, (b) Interfacial Area Concentration, (c) Bubble Velocity,

(d) Sauter Mean Diameter, and (e) Bubble Frequency

21 The Radial Profile Plots For $\mathrm{j}_{\mathrm{g}}=0.0965 \mathrm{~m} / \mathrm{s}$ and $\mathrm{L} / \mathrm{D}=8$,

(a) Void Fraction, (b) Interfacial Area Concentration, (c) Bubble Velocity,

(d) Sauter Mean Diameter, and (e) Bubble Frequency

22 The Radial Profile Plots For $\mathrm{j}_{\mathrm{g}}=0.0965 \mathrm{~m} / \mathrm{s}$ and $\mathrm{L} / \mathrm{D}=60$,

(a) Void Fraction, (b) Interfacial Area Concentration, (c) Bubble Velocity,

(d) Sauter Mean Diameter, and (e) Bubble Frequency

23 The Radial Profile Plots For $\mathrm{j}_{\mathrm{g}}=0.0384 \mathrm{~m} / \mathrm{s}$ and $\mathrm{L} / \mathrm{D}=8$,

(a) Void Fraction, (b) Interfacial Area Concentration, (c) Bubble Velocity,

(d) Sauter Mean Diameter, and (e) Bubble Frequency

24 The Radial Profile Plots For $\mathrm{j}_{\mathrm{g}}=0.0384 \mathrm{~m} / \mathrm{s}$ and $\mathrm{L} / \mathrm{D}=60$,

(a) Void Fraction, (b) Interfacial Area Concentration, (c) Bubble Velocity,

(d) Sauter Mean Diameter, and (e) Bubble Frequency 65

25 Comparison of Void Fraction Profiles at $L / D=8$ and $L / D=60$ for $j_{1}=1.0 \mathrm{~m} / \mathrm{s}$ and $i_{\mathrm{g}}=$ (a) 0.0965 , (b) 0.0696 , (c) 0.0384 , and (d) $0.0192 \mathrm{~m} / \mathrm{s} . .69$

26 Comparison of Interfacial Area Concentration Profiles at $L / D=8$ and $L / D=60$ for $j_{1}=1.0 \mathrm{~m} / \mathrm{s}$ and $j_{g}=$ (a) 0.0965 , (b) 0.0696 , (c) 0.0384 , and (d) $0.0192 \mathrm{~m} / \mathrm{s}$

27 Comparison of Bubble Velocity Profiles at $L / D=8$ and $L / D=60$ for $\mathrm{j}_{1}=1.0 \mathrm{~m} / \mathrm{s}$ and $\mathrm{j}_{\mathrm{g}}=$ (a) 0.0965 , (b) 0.0696 , (c) 0.0384 , and (d) $0.0192 \mathrm{~m} / \mathrm{s} . . \quad 73$

28 Comparison of Sauter Mean Diameter Profiles at $L / D=8$ and $L / D=60$ for $\mathrm{j}_{1}=1.0 \mathrm{~m} / \mathrm{s}$ and $\mathrm{j}_{\mathrm{g}}=$ (a) 0.0965 , (b) 0.0696 , (c) 0.0384 , and (d) $0.0192 \mathrm{~m} / \mathrm{s} . .75$ 
29 Comparison of Bubble Frequency Profiles at $L / D=8$ and $L / D=60$ for $j_{1}=1.0 \mathrm{~m} / \mathrm{s}$ and $j_{g}=$ (a) 0.0965 , (b) 0.0696 , (c) 0.0384 , and (d) $0.0192 \mathrm{~m} / \mathrm{s} . .77$

30 Comparison of Void Fraction Profiles at $L / D=8$ and $L / D=60$ for $j_{1}=0.6 \mathrm{~m} / \mathrm{s}$ and $j_{g}=$ (a) 0.0965 , (b) 0.0696 , (c) 0.0384 , and (d) $0.0192 \mathrm{~m} / \mathrm{s}$.. 79

31 Comparison of Interfacial Area Concentration Profiles at $L / D=8$ and $L / D=60$ for $j_{\mathrm{t}}=0.6 \mathrm{~m} / \mathrm{s}$ and $j_{\mathrm{g}}=$ (a) 0.0965 , (b) 0.0696, (c) 0.0384 , and (d) $0.0192 \mathrm{~m} / \mathrm{s}$

32 Comparison of Bubble Velocity Profiles at $L / D=8$ and $L / D=60$ for $\mathrm{j}_{\mathrm{l}}=0.6 \mathrm{~m} / \mathrm{s}$ and $\mathrm{j}_{\mathrm{g}}=$ (a) 0.0965 , (b) 0.0696 , (c) 0.0384 , and (d) $0.0192 \mathrm{~m} / \mathrm{s} . .83$

33 Comparison of Sauter Mean Diameter Profiles at $L / D=8$ and $L / D=60$ for $\mathrm{j}_{1}=0.6 \mathrm{~m} / \mathrm{s}$ and $\mathrm{j}_{\mathrm{g}}=$ (a) 0.0965 , (b) 0.0696 , (c) 0.0384 , and (d) $0.0192 \mathrm{~m} / \mathrm{s} . .85$

34 Comparison of Bubble Frequency Profiles at $L / D=8$ and $L / D=60$ for $\mathrm{j}_{1}=0.6 \mathrm{~m} / \mathrm{s}$ and $\mathrm{j}_{\mathrm{g}}=(\mathrm{a}) 0.0965$, (b) 0.0696 , (c) 0.0384 , and (d) $0.0192 \mathrm{~m} / \mathrm{s}$.. 87

35 Comparison of Void Fraction Profiles at $L / D=8$ and $L / D=60$ for $j_{1}=0.4 \mathrm{~m} / \mathrm{s}$ and $\mathrm{j}_{\mathrm{g}}=$ (a) 0.0965 , (b) 0.0696 , (c) 0.0384 , and (d) $0.0192 \mathrm{~m} / \mathrm{s}$.. 89

36 Comparison of Interfacial Area Concentration Profiles at $L / D=8$ and $L / D=60$ for $j_{1}=0.4 \mathrm{~m} / \mathrm{s}$ and $j_{8}=$ (a) 0.0965 , (b) 0.0696 , (c) 0.0384 , and (d) $0.0192 \mathrm{~m} / \mathrm{s}$

37 Comparison of Bubble Velocity Profiles at $L / D=8$ and $L / D=60$ for $j_{1}=0.4 \mathrm{~m} / \mathrm{s}$ and $j_{g}=(a) 0.0965$, (b) 0.0696 , (c) 0.0384 , and (d) $0.0192 \mathrm{~m} / \mathrm{s} . .93$

38 Comparison of Sauter Mean Diameter Profiles at $L / D=8$ and $L / D=60$ for $\mathrm{j}_{1}=0.4 \mathrm{~m} / \mathrm{s}$ and $\mathrm{j}_{\mathrm{g}}=$ (a) 0.0965 , (b) 0.0696 , (c) 0.0384 , and (d) $0.0192 \mathrm{~m} / \mathrm{s} . .95$

39 Comparison of Bubble Frequency Profiles at $L / D=8$ and $L / D=60$ for $\mathrm{j}_{1}=0.4 \mathrm{~m} / \mathrm{s}$ and $\mathrm{j}_{\mathrm{g}}=$ (a) 0.0965 , (b) 0.0696 , (c) 0.0384 , and (d) $0.0192 \mathrm{~m} / \mathrm{s} . .97$

40 Comparison of Void Fraction Profiles at $L / D=8$ and $L / D=60$ for $j_{1}=0.1 \mathrm{~m} / \mathrm{s}$ and $j_{8}=$ (a) 0.0965 , (b) 0.0696 , (c) 0.0384 , and (d) $0.0192 \mathrm{~m} / \mathrm{s} . .99$

41 Comparison of Interfacial Area Concentration Profiles at $L / D=8$ and $L / D=60$ for $j_{l}=0.1 \mathrm{~m} / \mathrm{s}$ and $j_{g}=$ (a) 0.0965 , (b) 0.0696, (c) 0.0384 , and (d) $0.0192 \mathrm{~m} / \mathrm{s}$ 101

42 Comparison of Bubble Velocity Profiles at $L / D=8$ and $L / D=60$ for $j_{1}=0.1 \mathrm{~m} / \mathrm{s}$ and $j_{g}=$ (a) 0.0965 , (b) 0.0696 , (c) 0.0384 , and (d) $0.0192 \mathrm{~m} / \mathrm{s} . .103$ 
43 Comparison of Sauter Mean Diameter Profiles at $L / D=8$ and $L / D=60$ for $j_{l}=0.1 \mathrm{~m} / \mathrm{s}$ and $j_{g}=(a) 0.0965$, (b) 0.0696 , (c) 0.0384 , and (d) $0.0192 \mathrm{~m} / \mathrm{s} \mathrm{..} 105$

44 Comparison of Bubble Frequency Profiles at $L / D=8$ and $L / D=60$ for $j_{1}=0.1 \mathrm{~m} / \mathrm{s}$ and $j_{g}=$ (a) 0.0965 , (b) 0.0696 , (c) 0.0384 , and (d) $0.0192 \mathrm{~m} / \mathrm{s} . .107$

45 The Flow Regime Map and present Data Points .............................................. 109

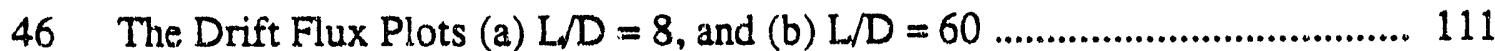

47 Comparison of Measurements from $L / D=8$ and $I / D=60$

(a) Void Fraction, and (b) Interfacial Area Concentration

48 The Average of Interfacial Area Concentration verse Void Fraction at near wall region for (a) $L / D=8$, and (b) $L / D=60$

49 The Average of Interfacial Area Concentration verse Void Fraction at core region for (a) $L / D=8$, and (b) $L / D=60$

50 The Average of Interfacial Area Concentration verse Void Fraction of the Pipe Cross-Section for (a) $L / D=8$, and (b) $L / D=60$

51 The Average of Sauter Mean Diameter verse the Average Void Fraction at (a) $L / D=8$, and (b) $L / D=60$

52 The Plot of $\alpha_{\text {peak }} /<\alpha>$ for (a) $L, D=8$, and (b) $L / D=60$ 
viii

\section{NOMENCLATURE}

$a_{i}$

$a_{i}(x, y, z, t)$

$\overrightarrow{a_{i}}$

A

A。

$\mathrm{C}_{\mathrm{D}}$

C。

$D_{\mathrm{sm}}$

$f(x, y, z, t)$

$f_{j}(x, y, z, t)$

g

$g(\alpha)$

h

$h_{\mathbf{l d}}$

$\mathrm{H}_{k}$

$<j>$

$\mathrm{j}_{\mathrm{g}}, \mathrm{j}_{\mathrm{l}}$

$\mathrm{m}_{*}$

$\mathbf{M}_{\mathrm{ik}}$

n

$\mathbf{n}_{j}$

$\mathbf{n}_{3}$
Interfacial area concentration

Local instantaneous interfacial area concentration

Time averaging of $a_{i}(x, y, z, t)$

Area of the test section

Area of the orifice

Friction coefficient of the orifice

Coefficient of the profile effect

Sauter mean diameter

Function representing an interface

Function representing jth interface

Acceleration due to gravity

Probability density function of $\alpha$

Height of the two-phase fluid column

Heat Transfer coefficient at interface

Enthalpy of $k$ phase

Average volumetric flux

Superficial velocity for gas and liquid

Mean mass transfer per unit area for $k$ phase

Interfacial force for $\mathrm{k}$ phase

Number

Unit normal vector of $j$ th interface

Unit vector in the direction of a probe 


\begin{tabular}{|c|c|}
\hline$N_{t}$ & Number of bubbles or droplets passing a point per unit time \\
\hline $\mathbf{N}_{w}$ & Number of bubbles or droplets used for velocity calculation \\
\hline Pr & Pressure of $k$ phase \\
\hline$P_{\text {cal }}$ & Pressure at the calibration of the flow meter \\
\hline$P_{\text {ind }}$ & Pressure of injection of the gas flow meter \\
\hline$P(\alpha, \beta, \mu, v)$ & Probability density function of $\quad \alpha, \beta, \mu, \nu$ \\
\hline $\mathbf{q}$ & Mean conduction heat flux \\
\hline $\mathbf{q}_{k}^{t}$ & Turbulent heat flux of $\mathrm{k}$ phase \\
\hline$q_{k i}^{\prime \prime}$ & Interfacial heat flux \\
\hline$Q_{8}$ & Volumetric flow rate of gas \\
\hline$Q_{\text {gin }}$ & Volumetric flow rate reading from the flow meter \\
\hline $\mathrm{R}_{\mathrm{B}}$ & Biasing resistance of the probe \\
\hline $\mathrm{R}_{\mathrm{L}}$ & Resistance of the conducting medium \\
\hline t & Time when average is taken \\
\hline$t_{\text {try }}$ & Tirue when jth bubble hits the downstream sensor \\
\hline tuf & Time when jth bubble clears the downstream sensor \\
\hline$t_{\text {umj }}$ & Time when jth bubble hits the upstream sensor \\
\hline$t_{\text {uff }}$ & Time when jth bubble clears the upstream sensor \\
\hline $\mathrm{T}$ & Time \\
\hline $\mathrm{T}_{\mathrm{i}}$ & Temperature of $\mathrm{k}$ phase \\
\hline$T_{k}$ & Bulk temperature of $\mathrm{k}$ phase \\
\hline $\mathbf{v}_{\mathrm{y}}$ & Velocity of jth interface \\
\hline$\left|\mathbf{v}_{i j}\right|$ & Value of jth interface velocity \\
\hline$\overline{v_{i z}}$ & Arithmetic mean of $v_{i j j}$ \\
\hline
\end{tabular}




$v_{b j j}$
$v_{k}$
$v_{s j}$
$v_{s x j}$
$v_{i x}, v_{i y}, v_{b z}$
$V_{g d}$
$V_{o x d}$
$Z$

Greek Symbols

$\begin{array}{ll}\alpha & \text { Void fraction } \\ \alpha_{k} & \text { Volume fraction of } k \text { phase } \\ \alpha_{j} & \text { Angle between } \mathbf{v}_{i j} \text { and } \mathrm{n}_{\mathbf{z}} \\ \alpha_{o} & \text { Angle defined by Eq. (35) } \\ \beta_{j} & \text { Angle between } \mathrm{n}_{\mathrm{y}} \text { and } \mathrm{v}_{\mathrm{ij}} \text { projection on } \mathrm{x}-\mathrm{y} \text { plane } \\ \gamma & \text { Area Ratio in orifice flow rate measurement } \\ \Gamma_{r} & \text { Mass generation of } \mathrm{k} \text { phase } \\ \delta(\eta) & \text { Delta function } \\ \Delta s & \text { Separation of two sensors of a double-sensor probe } \\ \Delta t_{j} & \text { Time lag of jth interface between two sensors } \\ \Delta \tau_{j} & \text { Time lag when upstream sensor is inside jth bubble } \\ \theta_{j} & \text { Angle between } \mathrm{n}_{\mathrm{z}} \text { and } \mathrm{n}_{\mathrm{j}}\end{array}$

Velocity of jth interface in z-direction

Velocity of $k$ phase

Velocity measured by double sensor probe

Velocity measured by double sensor probe in z-direction

$x, y, z$, component of $v_{i y}$

Drift velocity of the gas phase

Output voltage of the probe

Characteristic impedance of the sensor 


\begin{tabular}{|c|c|}
\hline$\mu_{j}$ & Angle between $n_{2}$ and $n_{j}$ \\
\hline$v_{j}$ & Angle between $\mathbf{n}_{\mathrm{y}}$ and $\mathbf{n}_{\mathrm{j}}$ projection on $\mathrm{x}-\mathrm{y}$ plane \\
\hline$\xi_{k}$ & Angle between $n_{s}$ and $n_{j}$ \\
\hline$\rho_{j}$ & Density of $k$ phase \\
\hline$\rho_{m}$ & Mixture density of a two-phase fluid \\
\hline$\sigma_{s}$ & Fluctuating component of $\left|\mathbf{v}_{x x j}\right|$ \\
\hline$\tau$ & Average interface shear for $k$ phase \\
\hline$\tau_{k}^{\mu}$ & Average viscous stress for $k$ phase \\
\hline$\tau_{k}$ & Turbulent shear stress for $k$ phase \\
\hline$\phi_{j}$ & Angle between $n_{j}$ and $v_{i j}$ \\
\hline$\Phi_{k}$ & Energy dissipation for $\mathrm{k}$ phase \\
\hline$\Omega$ & T'ime duration \\
\hline
\end{tabular}

Subscripts

1

Liquid phase

g

Gas phase

i

Value at interface

k

$k$ phase (gas or liquid) 


\title{
Interfacial Area and Two-Phase Flow Structure Development Measured by a Double Sensor Probe
}

\author{
by \\ Waihung Leung, Shripad T. Revankar, Yoshihika Ishii, and MamoruIshii
}

\begin{abstract}
The interfacial area concentration is paramount important for modeling the interfacial transfer terms in the two-fluid model. However, local interfacial data is not widely available due to experimental difficulties. Lacking local phasic knowledge, the two-fluid model cannot be used to its full potential. Especially in the dispersed two-phase flow, the phasic structure changes both in space and time. Due to the complicated phase structures, the distribution mechanism of the dispersed phase is not yet well understood. In the many studies of dispersed two-phase flow, authors paid much attention to two-phase flow turbulent model development in hopes that the phasic distribution mechanism might be resolved. However, the existence of the dispersed interfaces modify the local the turbulent eddies in the continuum phase. The knowledge of the interfacial geometry becomes indispensable for modeling any hydrodynamic effects on the dispersed phase distribution. Hence, the basic length scale for the interfacial geometries must be first studied in order to construct an accurate constitutive relations of the interface transport in mass, momentum and energy.

In this report, we studied the local phasic characters of dispersed flow regime both at the entrance and at the fully developed regions. Since the dispersed phase is distributed randomly in the medium and enclosed in relatively small interfaces, the phasic measurement becomes difficult to obtain. Local probe must be made with a miniaturized sensor in order to reduce the interface distortion. The double-sensor resistivity probe has been widely used in local void fraction and interface velocity measurements because the sensors are small in cumparison with the interfaces. It has been tested and proved to be an accurate local phasic measurement tool. In these experiments, a double-sensor probe was employed to measure the local void fraction and interface velocity in an air-water system. The test section was a $5.08 \mathrm{~cm}$ id by $3.75 \mathrm{~m}$ height Lucite pipe. The advantage of the transparent section is that
\end{abstract}


flow regime can be determined by visualization. Furthermore, local phasic measurements can be verified by photographic studies. We concentrated our study on the bubbly flow regime only. The local measurements were conducted at two axial locations, $L / D=8$ and 60 , in which the first measurement represents the entrance region where the flow develops, and the second measurement represents the fully developed flow region where the radial profile does not change as the flow moves along the axial direction. Four liquid flow rates were chosen in combination with four different gas injection rates. The superficial liquid velocities were $j_{l}=1.0,0.6,0.4$, and $0.1 \mathrm{~m} / \mathrm{s}$ and superficial gas velocities were $j_{s}=0.0965$, $0.0696,0.0384$, and $0.0192 \mathrm{~m} / \mathrm{s}$. These combinations put the two-phase flow well in the bubbly flow regime. In this sequence of phenomenological studies, the local void fraction, interface area concentration, sauter mean diameter, bubble velocity and bubble frequency were measured.

One of the prominent phasic characters of the bubbly flow is the near wall void peaking. The change dispersed phase distribution from entrance to fully developed region can give some clue of what causes the dispersed phase accumulating near the wall. The detail comparison of the radial profiles between the entrance and the fully developed region are presented. The comparison plots include radial profile of void fraction, interfacial area concentration, sauter mean diameter, bubble velocity and bubble frequency. Since there is not enough information for analyzing the local dispersed-phase distribution, the mean value of the profile are taken by averaging over the cross section of the pipe. The average phasic character plots are shown by the drift flux plots and the comparison plots of the average void fraction, interfacial area concentration, and sajuter mean diameter between the two locations ( $L / D=8$ and 60 ). Finally, the peak to average void ratio is plotted against the average void fraction to show the percentage of the dispersed phase accumulating near the wall region.

\section{Introduction}

The two-fluid model is regarded as the most accurate treatment of a two-phase flow system, because it describes the phases separately in terms of the phasic conservation equations in mass, momentum, and energy balance. Furthermore, the interaction between the two phases are modeled by the interfacial transfer terms. These terms couple the two sets of balance equations mathematically. It is expected that the two-fluid model can 
accurately predict mechanical and thermal inequilibrium cases in a two-phase system. Particularly, in transient or entrance flow, when phases are accelerating with respect to each others, the inertia terms of each phase have to be considered sepiarately. Henceforth, in these cases, the interfacial transfer terms must be modeled accurately and the local averaged phasic character rnust also be known in detail in order to build a sensible reodel to predict the flow. The locial instant description of the rwo fluid model is difticult to model, because it contains information of fast fluctuating fiel is and phasic discontinuities. Therefore, the average macroscopic properties of the phasic flow fields are the more desirable and practical way to express a two-prinse system. The choice of applying the average method is always associated to the measurement technique which one uses. In our experiment, a local probe is used. Since the probe records the flow fielc lluctuation in the time domain, the measured local phasic information is useful for the time average form of the local formulation of the two-fluid model.

A three-dimensional two-fluid model has been otcained by using temporal or statistical averaging [2]. The average form of the conservation equations removes the phasic discontinuities and gives the average interfacial transport description. The practical form of temporal or statistical average two-fluid equations were developed by Ishii (1975)

\section{Continuiry Equarion}

$\frac{\partial}{\partial t}\left(\alpha_{k} p_{k}\right)+\nabla \cdot\left(\alpha_{k} p_{k} \mathbf{v}_{k}\right)=\Gamma_{k}$,

\section{Momenoum Equation}

$$
\begin{aligned}
\frac{\partial}{\partial t}\left(\alpha_{k} \rho_{k} v_{k}\right)+\nabla \cdot\left(\alpha_{k} \rho_{k} v_{k} v_{k}\right)= & --\alpha_{k} \nabla p_{k}+\nabla \cdot \alpha_{k}\left(\tau_{k}^{k}+\tau_{k}\right)+\alpha_{k} \rho_{k} g \\
& +v_{k i} \Gamma_{k}+M_{i k}-\nabla \alpha_{k} \cdot \tau_{i j}
\end{aligned}
$$

\section{Enchalpy Eouation}

$$
\begin{aligned}
\frac{\partial}{\partial t}\left(\alpha_{k} p_{k} H_{k}\right)+\nabla \cdot\left(\alpha_{k} \rho_{k} H_{k} v_{k}\right)= & -\nabla \cdot \alpha_{k}\left(q_{k}+q_{k}^{\prime}\right)+\alpha_{k} \frac{D_{k}}{D_{t}} p_{k} \\
& +H_{k} \Gamma_{k}+q_{k i}^{\prime \prime} \alpha_{i}+\Phi_{k}
\end{aligned}
$$


Here $\Gamma_{k}, M_{i k}, \tau_{i}, q_{k}^{\prime \prime}$, and $\Phi_{k}$ are the mass generation, generalized interfacial drag, interfacial shear stress, interfacial heat flux, and dissipation respectively. The subscript $k$ denotes $k$ th phase, i.e. $k=g$ for gas or $k=l$ for liquid, and $i$ stands for the value at the interface. $a_{i}$ is the interfacial area per unit volume. Since the thickness of the interface is assumed to be infinitesimally stiall and it has no sources or sinks, the inflow and outflow of flux must be balanced. Then it will be easy to obtain the average local jump conditions as:

$$
\begin{aligned}
& \sum_{k} \Gamma_{k}=0 \\
& \sum_{k} M_{i k}=0 \\
& \sum_{k}\left(\Gamma_{k} H_{k i}+q_{k i}^{\prime \prime} a_{i}\right)=0
\end{aligned}
$$

Therefore, constitutive equations for $\mathbf{M}_{\mathrm{ll}}, q_{g i}^{\prime \prime} a_{i}$, and $q_{i j}^{\prime \prime} a_{i}$ are necessary for the interfacial transfer terms. The enthalpy interfacial transfer condition indicates that specifying the heat flux at the interface for both phases is equivalent to the constitutive relation for $\Gamma_{k}$, if the mechanical energy transfer terms can be neglected. This aspect greatly simplifies the development of the constitutive relations for interfacial transfer terms.

By introducing the mean mass transfer per unit area, $m_{k}$, th : interfacial mass transfer term can be defined as:

$\Gamma_{k}=a_{i} m_{k}$

the interfacial energy transfer term in Eq. (3) can be rewritten as:

$\Gamma_{k} \mathrm{H}_{k i}+q_{k i}^{\prime \prime} a_{i}=a_{i}\left(m_{k} \mathrm{H}_{k i}+q_{k i}^{\prime \prime}\right)$

The heat flux at the interface should be modeled as the driving potential for the energy transfer. Thus,

$q_{k i}^{\prime \prime}=h_{k i}\left(\mathrm{~T}_{i}-\mathrm{T}_{k}\right)$

where $T_{i}$ and $T_{k}$ are the interfacial and bulk temperatures based on the mean enthalpy. $A$ similar treatment of the interfacial momentum transfer term is also possible. In the view of 
Eq.(5), Eq.(6), and Eq.(7), the importance of the role of interfacial area concentration in the interfacial transfer terms becomes apparent. Thus, in general, the interfacial transfer terms are given in terms of the interfacial area concentration $a_{i}$ and driving force as:

$$
\text { (Interfacial Transfer Term) }=a_{i} \times(\text { Driving Force) }
$$

It is essential to make a conceptual distinction between the effects of these $a_{i}$ and the driving force term. The interfacial area concentration is defined as the total surface area per unit volume, in which it has the dimension as per unit length. It characterizes the interfacial geometry effects of the transfer mechanism. The driving force term characterizes the transfer mechanism between the phases. In terms of modeling this interfacial driving force, one must keep in mind that it must be expressed in an average form. In the other words, the individual interface geometry and interfacial forces are not important, but the mean values of these quantities are used for modeling the system's interfacial geometry and forces balance. Especially in the dispersed two-phase flow, the interfaces are scattered and they are fluctuating both in space and time. The average quantities are more practical way to present the macroscopic interfacial geometry of a two-phase mixture.

In dispersed twn-phase flow, the characteristic length scale for modeling the interfacial momentum transfer ter $m$ is the diameter of the individual bubble, droplet, or particle. One of the important length scales sauter mean diameter $D_{\mathrm{sm}}$ which is defined by [4]:

$D_{s m}=\frac{6 B_{d}}{A_{i}} \quad$,

where $B_{d}$ and $A_{i}$ are the volume and the surface area of a typical fluid particle respectively. The number density $N_{d}$ of the fluid particle is given by:

$N_{d}=\frac{\alpha_{d}}{B_{d}}$

$\checkmark$ here $\alpha_{d}$ is the dispersed phase volum : fraction. We can also define the interfacial area concentration $a_{i}$ by:

$a_{i}=\mathrm{N}_{d} \mathrm{~A}_{i}$ 
In terms of the dispersed phase volume fraction and the interfacial area ccncentration, the sauter mean diameter can be defined as:

$\mathrm{D}_{s m}=\frac{6 \alpha_{d}}{a_{i}}$

The sauter mean diameter relates to other length scales in dispersed flow and are discussed in reference [9].

The temporal or statistical average of the two-fluid model conservation equations are presented in this section. For engineering applications, the detailed local character of the void fraction and interfacial area concentration must first be obtained. Before we can measure these local parameters, the local interfacial area concentration must formulated in terms of measurable variables. Furthermore, the associated measurement techniques must be defined in order to make a meaningful local interpretation. In the following section, we will discuss the theoretical argument of the time average measurement of the local interfacial area concentration. Here, only the outline of the derivation is presented, for more details see references [2] and [5].

\section{Local Interfacial Area Concentration Measurement}

If a small control volume of a two-phase fluid mixture is drawn, the interfacial area concentration is defined as the average surface area per unit volume. However, in a dispersed flow this simple definition is not useful for practical applications because the interfaces are sauted into many closed boundaries of individual fluid particles, in which it is difficult to measure. The local instant interfacial area concentration in terms of measurable quantity must be obtained first. Now, considering only the bubbly flow, the interface of a bubble is represented by a surface function $f(x, y, z, t)=0$. The local instant interfacial area can be defined as [2]:

$a_{i}\left(x_{o}, y_{o}, z_{o}, t\right)=\left|\nabla f\left(x_{o}, y_{o}, z_{o}, t\right)\right| \delta\left(f\left(x_{o}, y_{o}, z_{o}, t\right)\right)$

where $(x, y, z)$ is an arbitrary position, and $\delta$ is the delta function defined as:

$\delta(\eta)=\left\{\begin{array}{ll}1 & \text { for } \eta=0 \\ 0 & \text { for } \eta=1\end{array}\right\}$ 
Eq.(13) shows that the instant local interfacial area concentration can be obtained if the instant local surface gradient is known. However, this gradient is impossible to measure by any practical mean. Furthermore, in a dispersed two-phase flow, the interfaces are not stationary. Mathematically, the delta function gives a pulse when an interface function goes to zero which means an interface exists at this location and time. In bubbly flow, at a fixed location, one observed a chain of pulses with values fluctuating discontinuously with time. It gives no useful information of the macroscopic interfacial character. Therefore, we must first find the measurable representation and then a temporal average local interfacial area concentratiou can be used. The time average interfacial area concentration is given by [2]:

$\vec{a}_{i}\left(x_{0}, y_{0}, z_{0}\right)=\frac{1}{\Omega} \sum_{j}\left\{\frac{\left|\nabla f_{j}\right|}{\left|\partial f_{j} / \partial t\right|}\right\} \quad$ at $\left(x_{0}, y_{o}, z_{o}\right)$

The advantage of using this time average interfacial area concentration formulation is that the local measurement technique can be implemented. One can obtain the relation [5]:

$\frac{\left|\nabla f_{j}\right|}{\left|\partial f_{j} / \partial t\right|}=\frac{1}{\left|\mathbf{v}_{i j} \cos \phi_{j}\right|}$

where $v_{i j}$ is the velocity of $j$ th interface and $\phi_{j}$ is the angle between the velocity of $j$ th interface and the surface unit normal as shown in Fig. 1. Eq.(16) shows the relation between the interface gradient and the interfacial velocity which is now a measurable quantity. Substituting Eq.(16) into Eq.(15), the time average of interfacial area concentration becomes:

$\bar{a}_{i}^{t}\left(x_{0}, y_{0}, z_{0}\right)=\frac{1}{\Omega} \sum_{j} \frac{1}{\left|v_{i j}\right| \cos \phi_{j}}=\frac{\left(\begin{array}{c}\sum_{j} \\ j\end{array}\right)}{\Omega}\left\{\frac{\sum_{j} \frac{1}{\left|v_{i j}\right| \cos \phi_{j}}}{\left(\begin{array}{c}\sum_{j} \\ j\end{array}\right)}\right\}$

Let $t_{j}$ be the time location where the sensor intercepted with the jth interface, $T$ be an arbitrary time when the sampling started, and $\Omega$ be the duration of the sampling, then sequential interface-interception events are marked by $T<\ldots . t_{f-1}<t_{j}<t_{j+1} \ldots . .<+\Omega$. Let $\tau$ be the average interval of the between events and it can be defined as:

$\lim _{n \rightarrow \infty} \frac{1}{2 n+1} \sum_{j=-n}^{n}\left|t_{j+1}-t_{j}\right|=\tau$ 


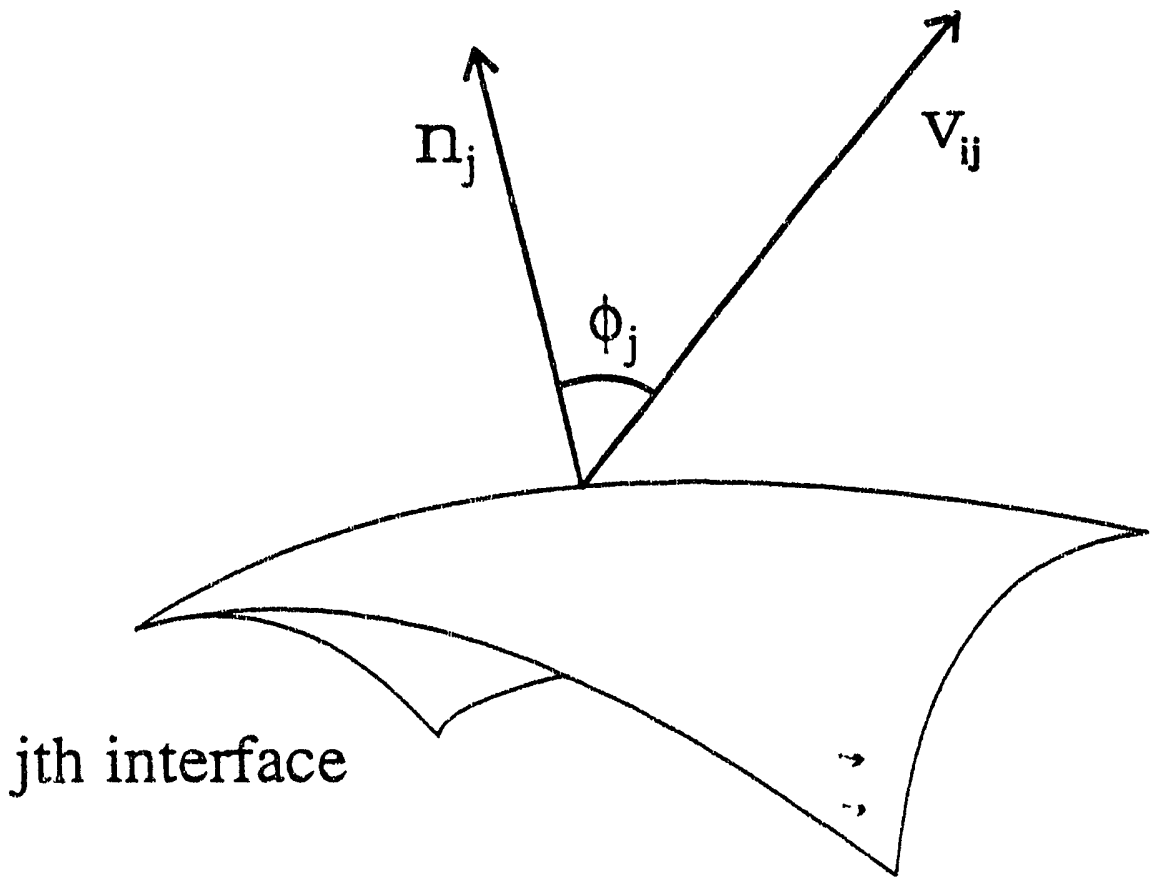

Fig. 1 Angle Between $\mathbf{v}_{\mathrm{ij}}$ and $\mathbf{n}_{\mathrm{j}}$ 
and for large $\Omega$, one can obtain the following relation:

$\left(\sum_{j}\right)=\frac{\Omega}{\tau}$

Substituting Eq.(19) into Eq.(17) yields the time average interfacial area concentration as:

$\vec{a}_{i}\left(x_{0}, y_{0}, z_{0}\right)=\frac{1}{\tau} \frac{1}{v_{i} \mid \cos \phi}$

The over bar denotes the arithmetic mean of the reciprosal of the interfacial velocity which is defined as:

$\frac{1}{\left|v_{i}\right| \cos \phi}=\frac{\sum\left(\frac{1}{\left|v_{i j}\right| \cos \phi_{j}}\right)}{\left(\sum_{j}\right)}$

Assuming $\mathrm{v}_{\mathrm{ij}}$ and $\cos \phi_{j}$ have no statistical correlation between them, the mean of the velocity and the mean of the angle can be evaluated separately:

$\overline{\frac{1}{\left|v_{i}\right| \cos \phi}}=\left(\frac{1}{\left|v_{i}\right|}\right)\left(\frac{1}{\frac{1}{\cos \phi}}\right)$

where

$\frac{\overline{1}}{\left|\mathbf{v}_{i}\right|}=\frac{\sum_{j}\left(\frac{1}{\left|\mathbf{v}_{i j}\right|}\right)}{\left(\sum_{j}\right)}$

and

$\frac{1}{\cos \phi}=\frac{\sum_{j}\left(\frac{1}{\cos \phi}\right)}{\left(\sum_{j}\right)}$

Let $\mathbf{v}_{\mathrm{ij}}$ be the velocity of and $n_{j}$ be the unit normal of jth interface (see Fig . 2 and 3 ). In a given co-ordinate system ${ }_{y}$ the velocity vector makes an angle $\alpha_{j}$ with the $z$-axis and its 


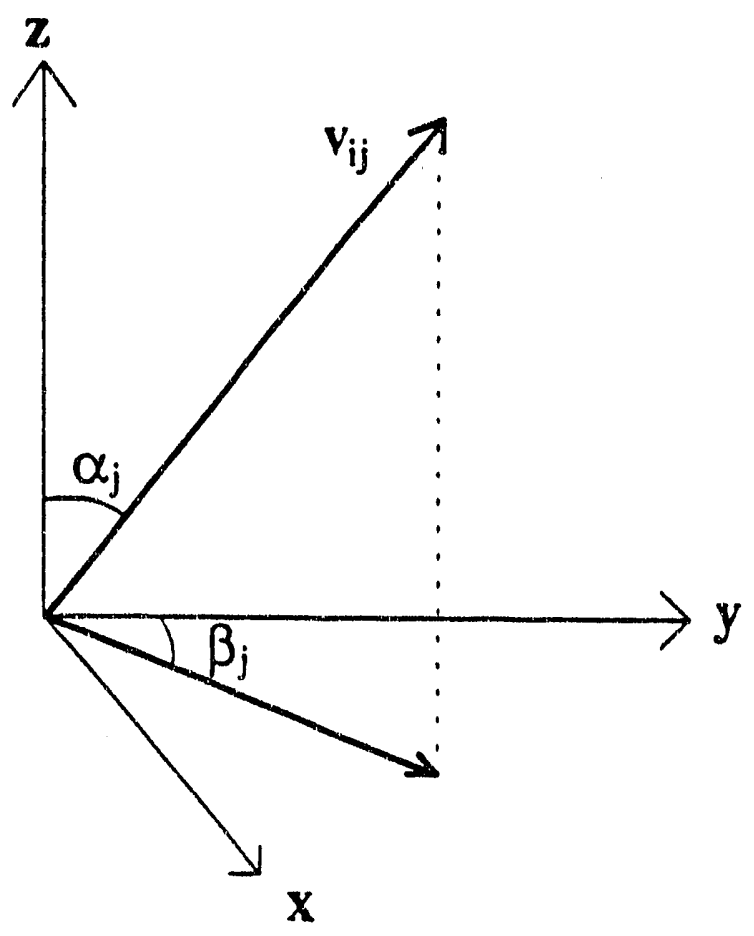

Fig. 2. The co-ordinate and angles of velocity vector

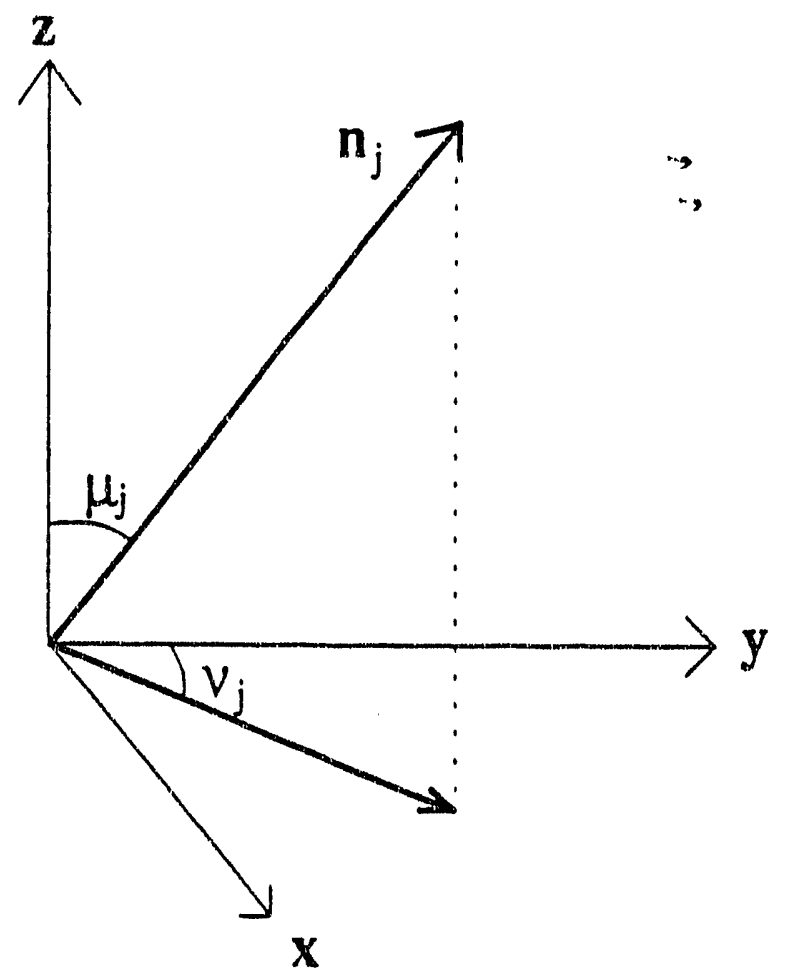

Fig. 3 The co-ordinate and angles of jth interface unit normal 
projection on the $x-y$ plane makes an angle $\beta_{j}$ with the $y$-axis. In the same system, the unit normal makes an angle $\mu_{j}$ with the $z$-axis and its projection on $x-y$ plane makes an angle $v_{j}$ with the $y$-axis. Then angle between $\mathbf{v}_{\mathrm{ij}}$ and $\mathbf{n}_{\mathrm{j}}$ can be expressed as:

$$
\begin{aligned}
\left|\mathbf{v}_{i j}\right| \cos \phi_{j} & =\mathbf{v}_{i j} \cdot \mathbf{n}_{j} \\
& =\left|\mathbf{v}_{i j}\right|\left(\cos \alpha_{j} \cos \mu_{j}+\sin \alpha_{j} \sin \mu_{j} \cos \left(\beta_{j}-\mu_{j}\right)\right)
\end{aligned}
$$

Substituting Eq.(24) and Eq.(25) into Eq.(22) and if the number of the sample is large, the summation can be approximated by an integral. Then, the harmonic mean of the normal interfacial velocicy is:

$$
\frac{1}{\left|\mathbf{v}_{i}\right| \cos \phi}=\frac{T}{\left|v_{i}\right|} \iiint \int \frac{\mathrm{P}(\alpha, \beta, \mu, v) d \alpha d \beta d \mu d v}{(\cos \alpha \cos \mu+\sin \alpha \sin \mu \cos (\beta-v))}
$$

where $P(\alpha, \beta, \mu, v)$ is the probability density function of $\alpha, \beta, \mu$, and $v$. However, this formulation requires a complete information of all three components of the velocity vector. A three double-sensor probe is needed to measure the velocities of each independent direction. Practically, this is difficult to implement due to the relatively large probe size compared to the bubble size and the limitations of the data acquisition system. Nevertheless, it may not be necessary to have all three components if the statistical behavior of the bubble velocity is known. In order to obtain a simple form of the density function, the bubbles are assumed to be perfect spheres, and the $x$ and $y$ component of $v_{i j}$ is random. Under these assumptions, $\beta$ and $v$ take on any value between 0 and $2 \pi$ with equal probability, and it is also reasonable to assume that $\beta$ and $v$ are statistically independent of each other. Furthermore, the two-phase mixture is flow mainly in the z-direction. In other words, the $z$-component of $\mathbf{v}_{\mathrm{ij}}$ is large in comparison with the $\mathrm{x}$ - and $\mathrm{y}$-direction velocity components, therefore, it means that $\alpha$ shall be small. Then the probability density function can be reduced to:

$P(\alpha, \beta, \mu, v)=\frac{1}{\pi} g(\alpha) \sin \mu \cos \mu d \alpha d \mu d(\beta-v)$

where

$$
g(\alpha)=\left\{\begin{array}{ccc}
1 / \alpha_{0} & \text { for } & 0 \leq \alpha \leq \alpha_{o} \\
0 & \text { for } & \alpha_{0} \leq \alpha \leq \pi / 2
\end{array}\right\}
$$




\section{Double Sensor Probe}
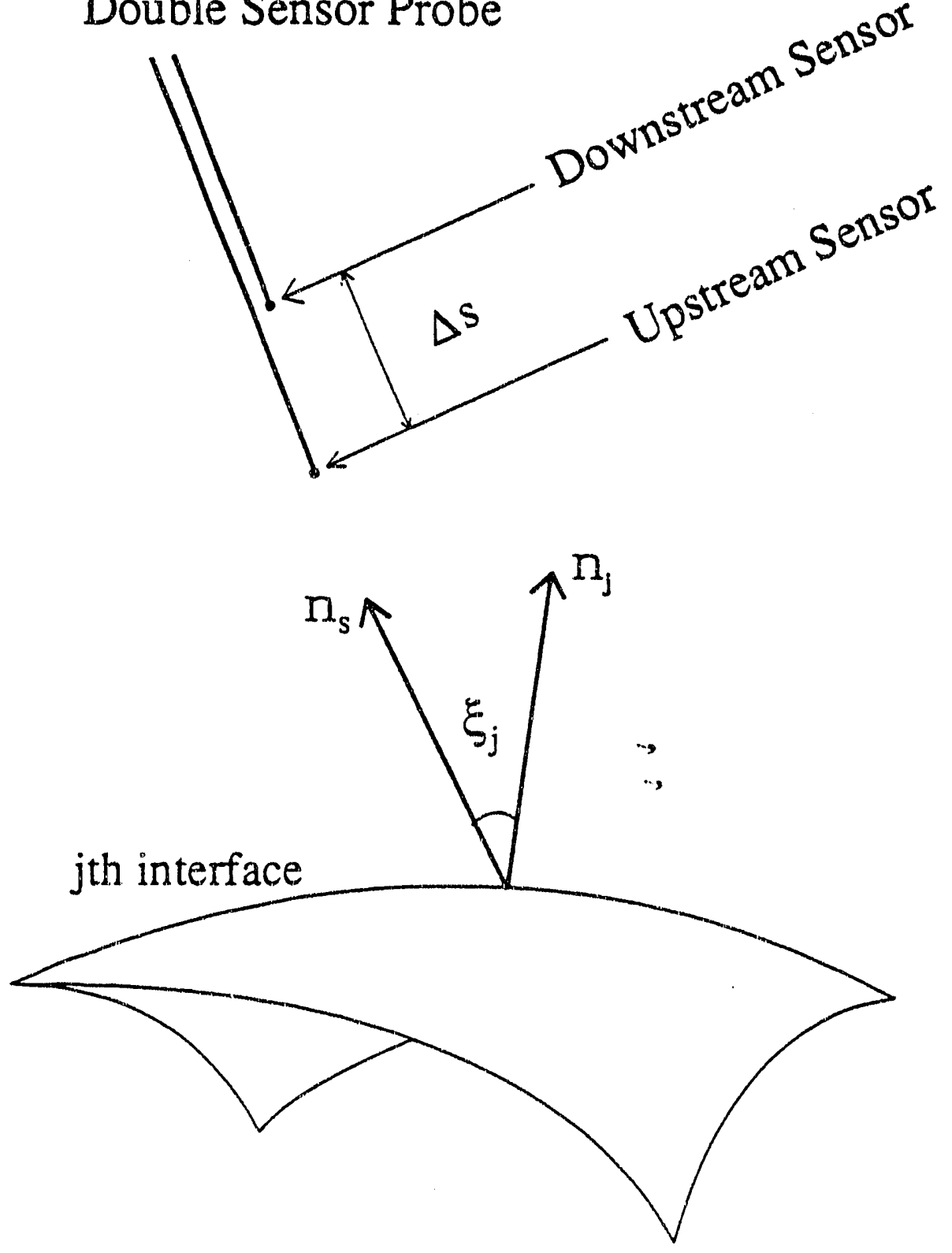

Fig. 4 Double Sensor Probe and jth interface 
where $\alpha_{0}$ is maximum angle that $v_{1 j}$ makes with the $z$-axis. The above statement means that $\alpha$ is randomly distributed between 0 and $\alpha_{0}$. Before we discuss how to determine $\alpha_{0}$, let's first talk about the actual velocity measurement by a single double sensor probe.

The orientation of $v_{i j}$ is described by $P(\alpha, \beta, \mu, v)$. The probability density function is determined by the velocity fluctuation in the main flow direction. Let $\mathbf{n}_{\mathbf{s}}$ be the unit vector where the upstream and downstream sensor are aligned (see Fig. 4), and it makes an angle $\xi_{j}$ with the unit normal of $\mathbf{n}_{j}$ and the jth interface. The two sensors are separated by $\Delta s$ and it is small. Then $\mathbf{v}_{\mathbf{3 j}}$ is the measured velocity associated with the jth interface, which is definei' as:

$\mathrm{v}_{s j}=\frac{\Delta \mathrm{s}}{\Delta t_{j}}$

where $\Delta t_{j}$ is the time lag of jth interface intercepting the up and down stream sensors. If $\mathbf{n}_{\mathbf{s}}$ is aligned with the $z$-axis (Eg. $\mathbf{n}_{\mathbf{s}}=\mathbf{n}_{\mathbf{z}}$ ), the measured interfacial velocity is $\mathbf{v}_{\mathbf{s z j}}$. The relation between the measured velocity and the normal interface velocity of jth interface is:

$\left|\mathbf{v}_{s j}\right| \cos \xi_{j}=\left|\mathbf{v}_{i j}\right| \cos \phi_{j}$

Since the probe is in the z-direction, one can easily see that from Fig.4 this relation becomes:

$\cos \xi_{j}=\cos \phi_{j}$

Assuming no statistical correlation between $v_{i j}$ and $\mu_{j}$, then the measured $v_{s z j}$ and the interfacial velocity $\mathbf{v}_{\mathrm{ij}}$ are related by:

$\frac{\sum_{j}\left(\frac{1}{\left|v_{s a j}\right|}\right)}{\left(\begin{array}{c}\Sigma \\ j\end{array}\right)}=\left\{\frac{\sum_{j}\left(\frac{1}{\mid v_{i j}}\right)}{\left(\begin{array}{c}\Sigma \\ j\end{array}\right)}\right\} \iiint \int \frac{P(\alpha, \beta, \mu, v) \cos \mu d \alpha d \beta d \mu d \nu}{(\cos \alpha \cos \mu+\sin \alpha \sin \mu \cos (\beta-\mu))}$

In the view of Eq.(32), Eq.(25) and Eq.(15), the time average interfacial area concentration is given in terms of measured interfacial velocities. Then the probability density function can be expressed as: 
$\vec{a}_{i}\left(x_{0}, y_{0}, z_{0}\right)=\frac{1}{\tau}\left\{\frac{\sum_{j}\left(\frac{1}{\left|v_{z j}\right|}\right)}{\left(\sum_{j}\right)}\right\} \frac{\iiint \int \frac{P(\alpha, \beta, \mu, v) d \alpha d \beta d \mu d v}{(\cos \alpha \cos \mu+\sin \alpha \sin \mu \cos (\beta-v))}}{\iiint \int \frac{P(\alpha, \beta, \mu, v) \cos \mu d \alpha d \beta d \mu d v}{(\cos \alpha \cos \mu+\sin \alpha \sin \mu \cos (\beta-v))}}$

where $0 \leq \alpha, \mu \leq \pi / 2$, and $0 \leq \beta, \quad v \leq 2 \pi$.

Substituting the probability density function into Eq.(33), then carrying out the integration, one can obtain the following result:

$\vec{a}_{i}\left(x_{o}, y_{o}, z_{o}\right)=\frac{4 \mathrm{~N}_{t} \sum_{j}\left(\frac{1}{\nabla_{o \neq 1}}\right) /\left(\sum_{j}\right)}{1-\cot \frac{\alpha_{o}}{2} \ln \left(\cos \frac{\alpha_{o}}{2}\right)-\tan \frac{\alpha_{o}}{2} \ln \left(\sin \frac{\alpha_{o}}{2}\right)}$

The resulting equation states that the time average locai interfacial area concentration can be computzd if values of $v_{\mathrm{kzz}}, \mathrm{N}_{\mathrm{t}}$ and $\alpha_{0}$ are given. The harmonic mean of $\mathrm{v}_{\mathrm{szz}}$ and $\mathrm{N}_{\mathrm{t}}$, number of bubbles per second, are obtained directly from the experiment. $\alpha_{0}$ can be estimated from the statistical parameters of the interfacial velocity. The basic assumptions are that the bubble is spherical and the probe intercepts the upper hemisphere of the bubble randomly, and this random process of the liquid phase turbulent leads to the result that the fluctuation of the $v_{i x}, v_{i y}$, and $v_{k z}$ are homogeneous, and $\alpha_{o}$ is given by [5]:

$$
\frac{\sin 2 \alpha_{0}}{2 \alpha_{o}}=\frac{1-\left(\sigma_{z}^{2} /\left|\overline{\mathbf{v}_{i z}}\right|^{2}\right)}{1+3\left(\sigma_{z}^{2} /\left|\overline{\mathrm{v}_{i z}}\right|^{2}\right)}
$$

where $\sigma_{z}$ is the standard deviation of $\mathbf{v}_{\mathrm{i}}$, which is defined as:

$$
\sigma_{2}^{2}=\frac{1}{\left(\sum_{j}\right)} \sum_{j}\left(v_{i z j}-\overline{v_{i z}}\right)^{2}
$$

Thus knowing the average velocity in the $z$-direction and the average fluctuation, $\alpha_{0}$ can be determined. Henceforth the time averaged local interfacial area concentration can be calculated by the relations given by Eq. (34) and Eq.(35). The double-sensor probe measures the $z$-direction interface velocity $v_{b z}$ and $\sigma_{z}$ is obtained by Eq.(36). 
The derivation of the time average local interfacial area concentration in terms of measurable quantities is presented. The question is whether the time average value is truly the local phasic character of the two-phase system. This can be answered by the ergodic theorem, which shows that if a system is stationary, the time average is equivalent to the spatial average. Therefore the measurement in the time domain can give the correct description of the spatial phasic character. The detailed argument of the ergodic hypothesis can be found in references [2] and [5].

\section{The Local Measurement Method}

The resistivity probe is widely used for local void fraction and bubble velocity measurement. It was first introduced by Neal and Bankoff [10] in measuring bubble size and velocity. The double-sensor resistivity probe was also used by Park et al.[11], Rigby et al.[12] for determination of the bubble parameters in three-phase fluidized beds; by Hoffer and Resnick [13] for finding the steady and unsteady state measurement in a single bubble system; by Serizawa et al. [23], Herringe and Davis [19] for studying the local phasic profiles development of air-water two-phase flow. Veteau [16], Burgess and Calderbank [14], Sekoguchi et al.[17], Kataoka and Serizawa [5] and Buchholz et al.[18] measured bubbly flow characteristics and the local specific interfacial area in gas-liquid systems. Ishii and Revankar [8] used the double-sensor and multi-sensor probe to measure the local void fraction and interfacial area concentration, verifying the average values by the pressure drop measurement and the photographic technique. In most of the cases, the double sensor probe is aligned with the mean flow direction, therefore, only the axial component of the velocity is measured. The theoretical principle of the local interfacial area concentration measurement was given by Kataoka et al.[5] and was outlined in the previous section.

The basic principle of the resistivity probe measurement is quite simple. More detail of how it works will be given in next section. Basically, when the sensor is resident in a different medium, it gives a different voltage output (see Fig. 5). The high output voltage indicates the sensor is inside a gas medium while low output voltage indicates the sensor is inside a liquid medium. The time trace of the sensor signal is also shown in Fig. 5. First, we look at the upstream sensor signal alone. If the jth bubble hits the upstream sensor, the 


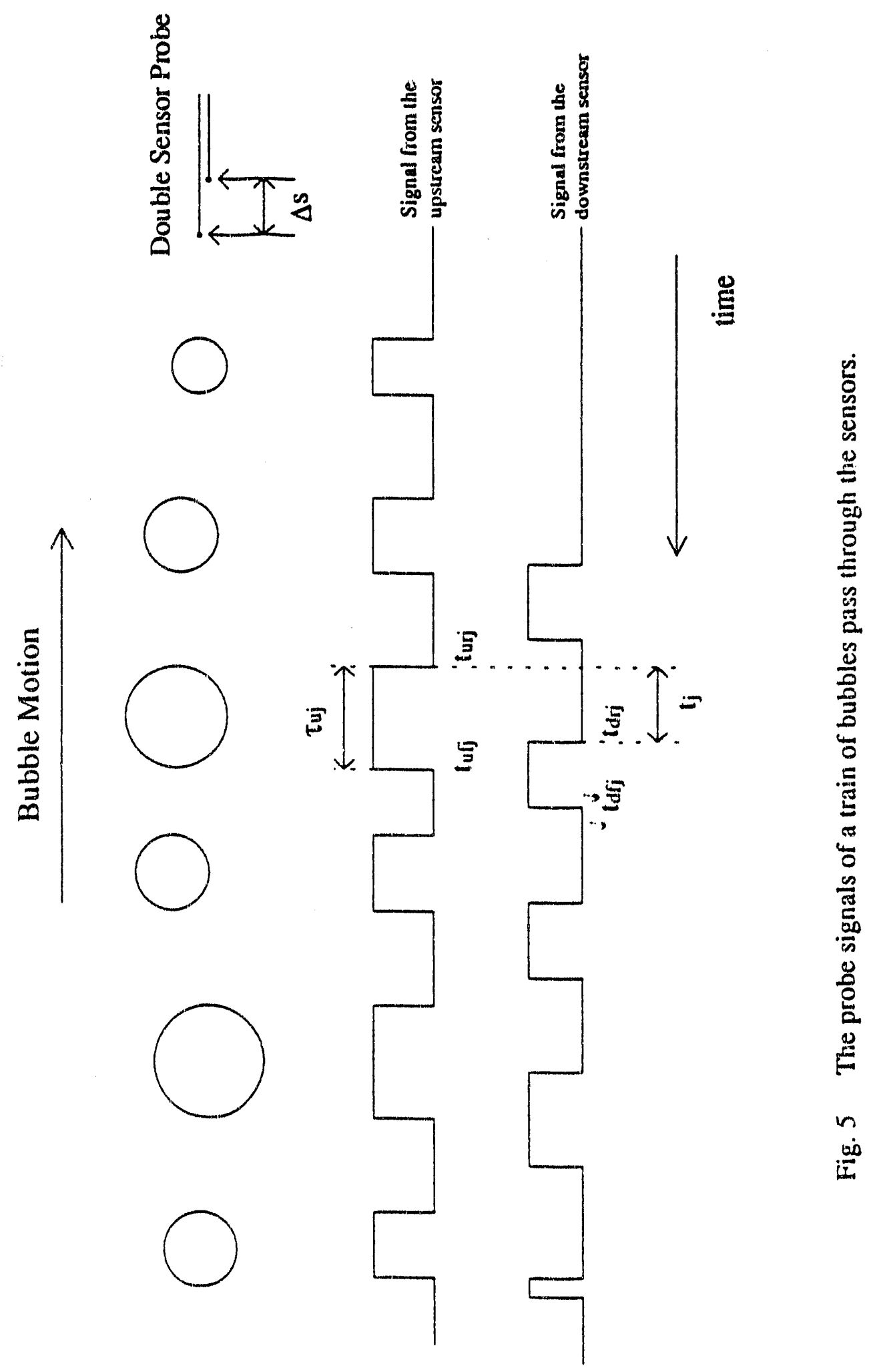


tirne rnarks $t_{\text {ury }}$ as the instant when the voltage jumps from low to high and $t_{\text {un }}$ as the instant when the voltage jumps from high to low. Then $\Delta \tau_{j}$ is defined to be the time of the sensor spending inside the juh bubble:

$\Delta \tau_{j}=\left|t_{\text {wrj }}-t_{\text {urj }}\right|$

If $\Omega$ is the total sampling time and $\mathrm{N}_{2}$ is the total number of bubbles detected by the upstream sensor, the void fraction can be defined as:

$\alpha\left(x_{0}, y_{0}, z_{0}\right)=\frac{1}{\Omega} \sum_{j=0}^{N_{j}} \Delta \tau_{j}$

where $\left(x_{0}, y_{0}, z_{0}\right)$ is the upstream sensor location. Eq. (38) gives the value of the tirne average local void fraction. Only the upstream sensor signal is used for evaluating the local void fraction. The downstream sensor usually gives a lower value of void fraction because the upstream sensor hinders the bubble reaching the downstream sensor. Thus, the void fraction measurement from the downstream sensor is not arcurate at all.

The previous section shows that the time average local interfacial area concentration can be computed from the measurement of the axial interfacial velocity. The double sensor probe is equipped to handle the velocity measurement. The downstream sensor is located at $\left(x_{0}, y_{0} z_{0}+\Delta s\right)$. If $\Delta t_{j}$ is the time difference of the jth interface hitting the upstream and downstream sensors, then the velocity of the interface can be measured as:

$v_{i a j}=\frac{\Delta s}{\Delta t_{j}}$

Let $N_{w}$ be the total number of bubbles hitting both the upstream and downstream sensor, then the harmonic mean of the axial velocity is given as:

$\overline{\frac{1}{\left|v_{i z}\right|}}=\frac{1}{N_{t v}} \sum_{i=0}^{N_{t w}} \frac{1}{v_{i z j}}$

We also need the mean axial velocity which is given by:

$$
\overline{v_{i s}}=\frac{1}{N_{r v}} \sum_{j=0}^{N_{r v}} V_{i z j}
$$


The standard deviation is computed by Eq. (35). Then $\alpha_{0}$ can be obtained by iterating on Eq.(36) until the left hand side is equal to the right hand side. The time average local interfacial area concentration is given by:

$\vec{a}_{i}\left(x_{0}, y_{0}, z_{0}\right)=\frac{4 N_{t} \sum_{j}\left(\frac{1}{\left|\gamma_{s i}\right|}\right) /\left(\sum_{j}\right)}{1-\cot \frac{a_{0}}{2} \ln \left(\cos \frac{\alpha_{0}}{2}\right)-\tan \frac{a_{0}}{2} \ln \left(\sin \frac{\alpha_{0}}{2}\right)}$

In real measurement, there is no guarantee that the bubble intercepting the upstream sensor must hit the downstream sensor or vice verse. The reason for this is that the sensors are separated in a finite distance and a bubble is free to go in any direction. The nonassociated signals cause error in velocity measurement. A criterion must be set to ensure the sigmals from the downstream sensor represents the same interface that hit the upstream sensor in earlier time.

1) Minimun waiting time

Since the interface cannot be intercepting both sensors at the same time, a time interval must be waited by the downstream sensor. The maximum velocity that a bubble can travel is the shortest waiting time that the downstream sensor detects the same interface.

2) Maximum waiting time

When the slowest bubble hits the upstream sensor, it takes the longest waiting time for the downstream sensor to detects the same interface.

3) Compatible resident time

The resident time of a bubble in the upstream sensor and downstream sensor should be comparable (30\%) to ensure that the sensors detect the very same bubble. Hence the widths of the rectangular pulse from both sensors must satisfy the condition:

$$
\frac{\left|\left(t_{w f i}-t_{w r j}\right)-\left(t_{d j j}-t_{d r j}\right)\right|}{\left(t_{u f j}-t_{u r j}\right)} \geq 30 \%
$$

The sauter mean diameter, defined in section 3, takes on the spatial average form. Now we use the definition provided by Eq. (12) but the local time average value is used instead: 


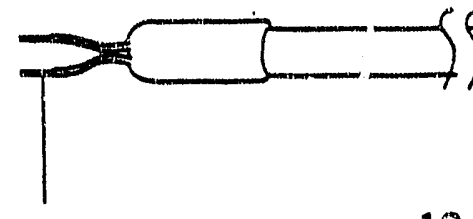

\section{Electric leads}

12.5mm o.d.

(0.12 $\mathrm{mm}$ brass wires) Brass Tube

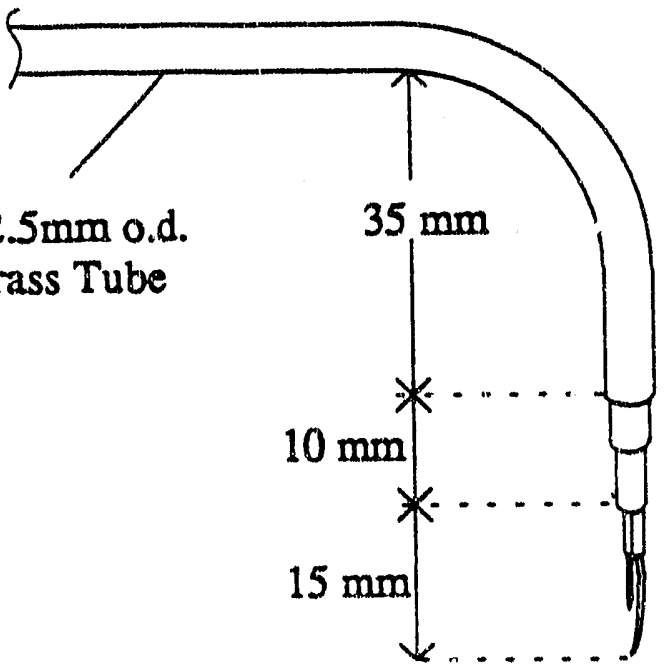

Fig. 6 Double Sensor Probe Design Schematic

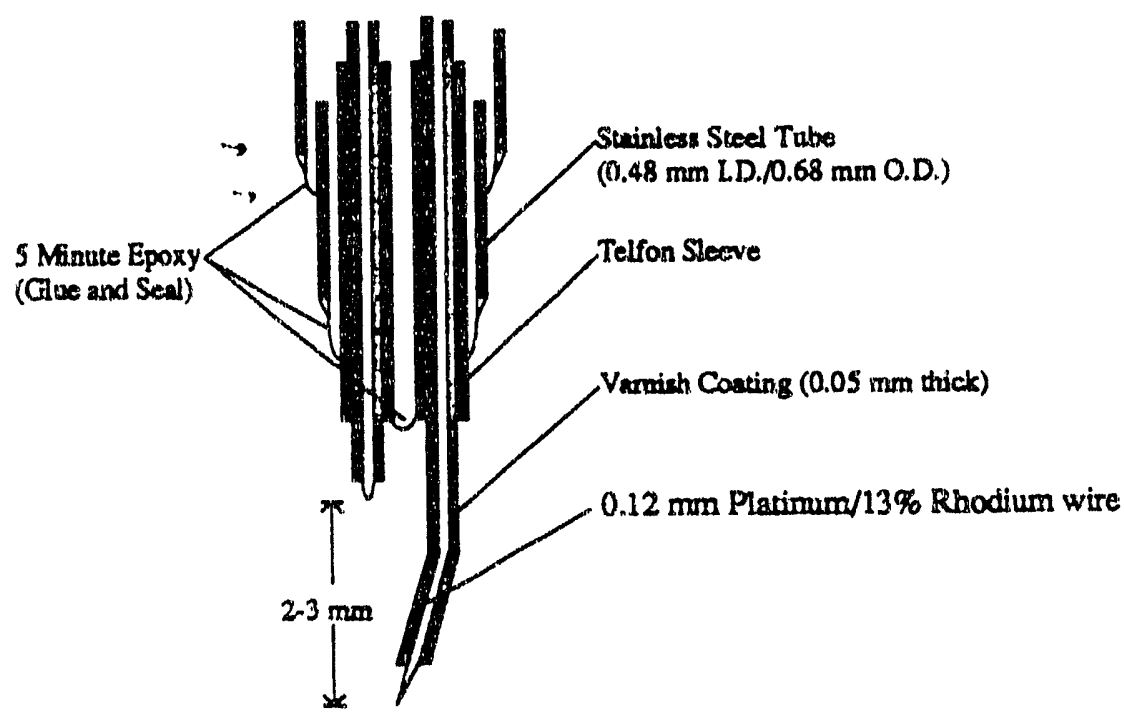

Fig. 7 The Sensors Design Schematic 
$D_{s m}\left(x_{o}, y_{o}, z_{o}\right)=\frac{6 \alpha\left(x_{o}, y_{o}, z_{o}\right)}{\bar{a}_{i}^{t}\left(x_{o}, y_{o}, z_{o}\right)}$

where the void fraction and interfacial area are obtained from the local temporal average measurement. In this case, it is the local mean diameter of the bubble at location $\left(x_{0}, y_{0}, z_{0}\right)$.

\section{Local Instrumentation}

\subsection{Double Sensor Probe}

The principle of resistivity probe is very simple. The sensor makes an electrical contact to the surrounding fluid. If the surrounding fluid is a conducting medium, it connects the sensor to the ground. Thus, the probe output voltage is proportional to the ratio of the medium resistivity and the biasing resistor (see Fig.8). Since different fluids have different characteristic resistivity, the output voltage changes according to the resident fluid between the sensor and the ground. In this way the different phases can be detected according to the output voltage if the fluid resistivities are known. In an air-water system, water is a good conductor compared to air. This system is much simpler than the multiphase systern because there are only two distinct voltage levels. The Equivalent electric circuit is shown in Fig.8. Resistivity probes can be used for the bubbly flow local phasic measurement.

\subsection{Probe Design and Biasing}

The sensor basically provides the electrical contact between the external conducting medium and the biasing circuit. Thus, it must be made by good conducting materials such as brass, gold or platinum. On the other hand, the sensor wire must be thin but stiff in order to pinch through the bubble interface without creating much distortion. Furthermore, the water environment is very corrosive. The sensor tip must be a metal alloy which is electrochemically stable. In the past, the sensor was made by a stainless steel needle. Due to the electrochemical effect, the sensor tip could be completely dissolved in the water within two hours. We find that a platinum wire with $13 \%$ rhodium is a good choice. A thin wire provides enough strength and is corrosion proof in a water environment. Most of the probes that we used in the experiments could last more than a month and the failure was usually caused by the break down of the insulating varnish. The sensor design is shown in Fig.7. 


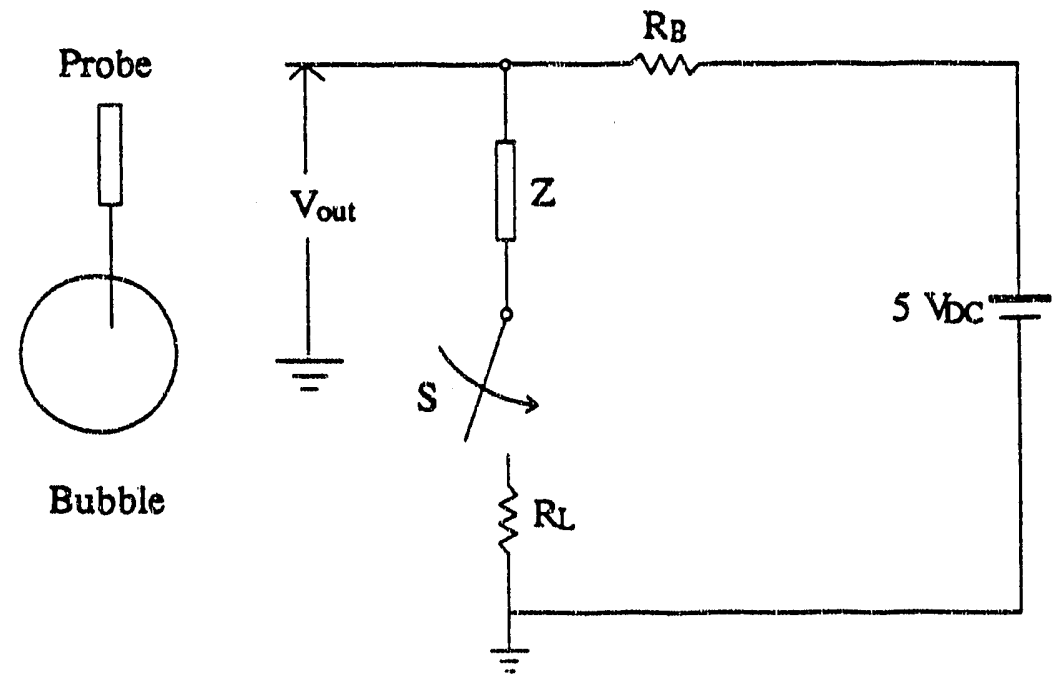

Fig.8 The Equivalent Circuit of the Resistivity Probe.

( $\mathrm{S}$ is opened if sensor states in gas phase, and

$S$ is closed if sensor states inside Liquid phase)
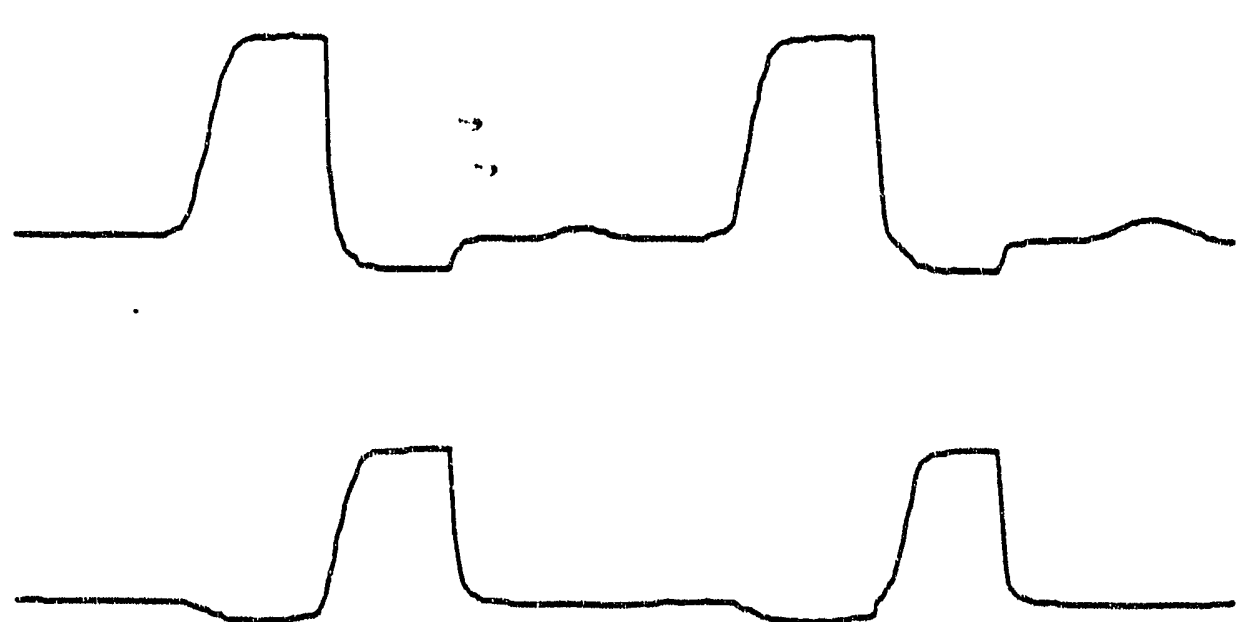

Fig 9. The Trace of the double-probe signals ( On top is the signal from the up-stream sensors and the first pulse is roughly $3.3 \mathrm{~ms}$, and at the bottom is the signal from downstream sensor)) 
It is made by the platinum alloy wire with diameter $0.12 \mathrm{~mm}$ and insulated by the vamish to the very tip. Then the insulation is stripped away by a razor blade and the exposed length is roughly 2 to 3 times the diameter. The bare tip makes the electrical contact to the water.

The design of the double sensor probe is shown in Fig.6. The sensor is inserted into a Teflon sleeve to ensure that it is well insulated from the surrounding metal parts and the sensors must be totally insulated from each other to avoid cross interference. The two sensors are hold together and keep in position by a small stainless tube. Then the whole unit is inserted into other tubes with larger diameters until it can fit into a brass tube with 3.175 $\mathrm{cm} O D(1 / 8 ")$. The larger brass tube gives a stronger structural support to the sensors in a flow. The metal tubes and the sensors are glued together by epoxy which is also the water seal of the probe. The miniaturized sensors reduce the obstruction of the flow and they can detect the bubble interfaces with minimum distortion. The electric connection is made by soldering the other end of the sensor wire to a copper wire lead. The detailed dimensions of the probe is given in Fig.6.

The biasing circuit that is used in this experiment is the simplest one (see Fig.8), A 5 $V_{D C}$ source supplies the biasing voltage. An adjustable resistor $R_{B}$ is connected in series with the sensor $\mathrm{Z}$; where $\mathrm{Z}$ is the characteristic impedance of the sensor. The bias resistor is adjusted to give an optimum output signal voltage. Between the sensor and the ground is the fluid media which is modeled by a simple resistor $R_{L}$. The $V_{\text {oul }}$ is the voltage reading between the sensor and the ground. The switch $S$ represent the state of surrounding medium. If the sensor is resident in the liquid phase, the circuit is closed and the voltage output is low. This is the same as having switch $\mathrm{S}$ closed in the equivalent circuit. When a gas bubble hits the sensor, the circuit continuity is broken. Then the output registers a high voltage which is the same as having $S$ open in the equivalent circuit. The voltage output difference discriminates the phases passage of the local point in time domain. Thus the history of the local phasic character is recorded. Let $\Delta s$ be the separation of the two sensors and $t_{j}$ be the time lag of the jth interface hitting the downstream sensors after it passed the upstream sensor, the interfacial velocity is then obtained by Eq.(26).

\subsection{Signal Processing}

The wetting and dry-out of the sensor due to the surface tension effect case the signal delay of the interface detection. Furthermore, if the electrical voltage changes stepwise, the circuit does not respond instantaneously. A time delay is characterized by a fixed time 
constant which is related to the probe impedance. We can lump both effect together and assign a characteristic impedance ( $Z$ in Fig.8.) If the time constant is large, the rise time or the falling time will be a significant part of the signal itself. The traces of the probe signals are shown in Fig.9. We can see that the rise time of the signal is much longer than the falling time. The transition from liquid phase to the gas phase shows the voltage signal delay much longer, in which it means the dry-out time is much longer. Any signal processing scheme must account for the portion of the rise time to ensure the void fraction measurement is correct. In our experiments, the signals are digitized and stored in the computer by a MetraByte DAS-20 data acquisition system. A threshold level is set to be the mid-level of the voltage difference in order to signify an interface hit. However, the location where the interface hit has traced back to a point where the voltage rises to $10 \%$ above the noise level. Then the signals are rectified to a train of rectangular pulses containing only 1 's and 0 's and they are stored and processed by a computer program. " 1 " represents the gas phase and " 0 " represents the liquid phase. The processing subroutines are implemented by using Eq.(9), Eq.(32), Eq.(33), Eq.(34), and Eq.(35) and the theoretical arguments are given in section 3. The output of the program gives the results of time average local void fraction, interfacial area concentration, sauter mean diameter, interface velocity, and bubble frequency.

\section{The Test Section and Instrumentations}

\subsection{The Test Section}

A schematic diagram of the air-water loop is shown in Fig.10. The test section is made of a Lucite extruding tube. The tube is $5.08 \mathrm{~cm}$ id and $3.75 \mathrm{~m}$ height. The advantage of using the transparent material is that the flow visualization is possible and photographic technique can be used to verify the local measurements [8]. The loop is capable of experiments for both upward flow and also downward flow. In this report, we only consider the upward co-current flow cases. Water is injected into the mixing house where the bubble generator is located. A pump is used to provide the necessary forced convection flow.

The bubble generator is made of a sintered stainless steel tube with a porous wall and the porous size is 40 microns (see Fig.11). The size of the bubble generator is $2.54 \mathrm{~cm}$ id and $1.5 \mathrm{~cm}$ in height. Air is injected through the lower chamber from a pressurized storage tank. The chamber acts as a buffer to avoid any sudden pressure fluctuation. Bubbles are grown on the porous wall and sheared off by the water flow near the generator. After mixing 

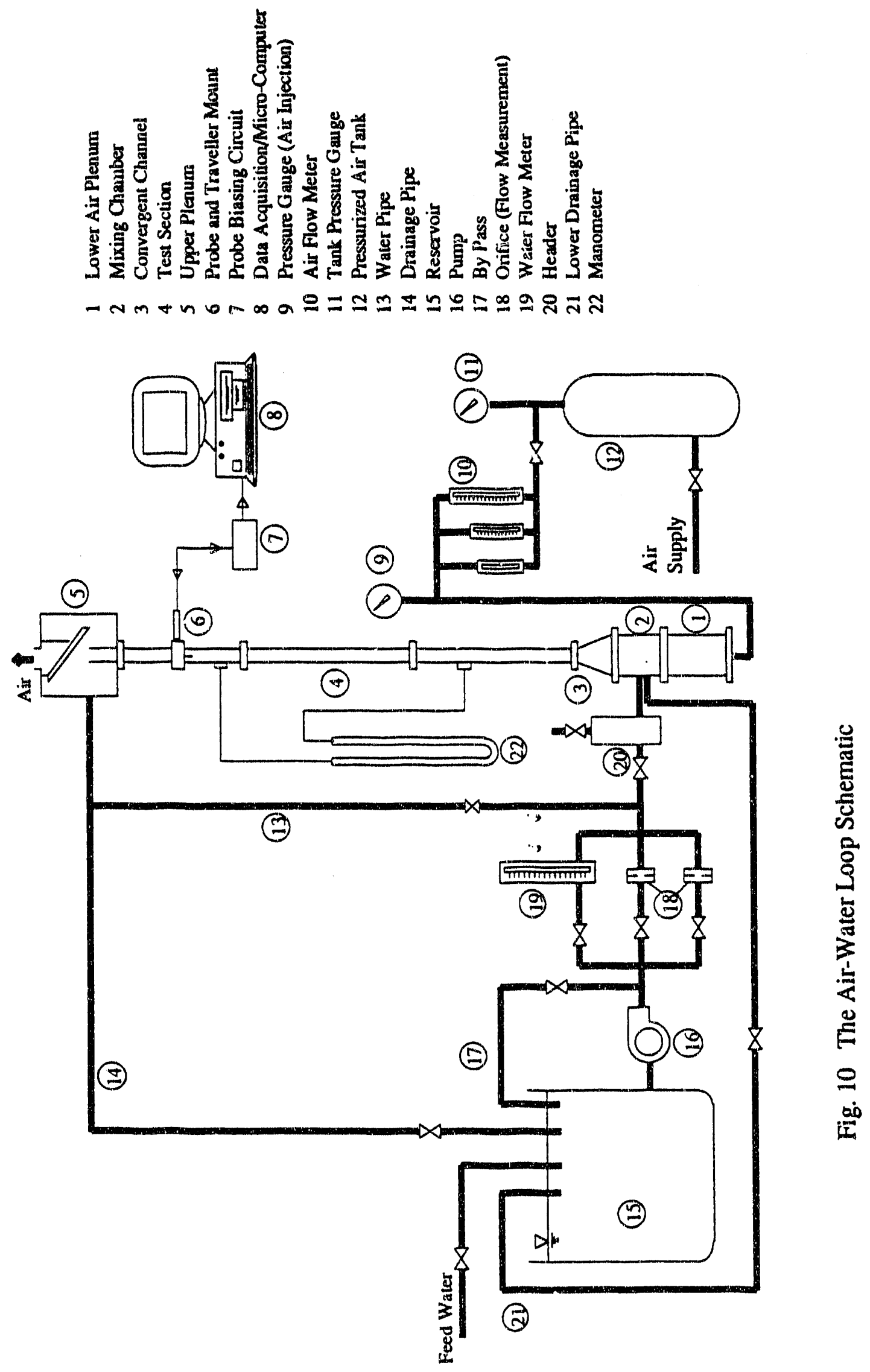


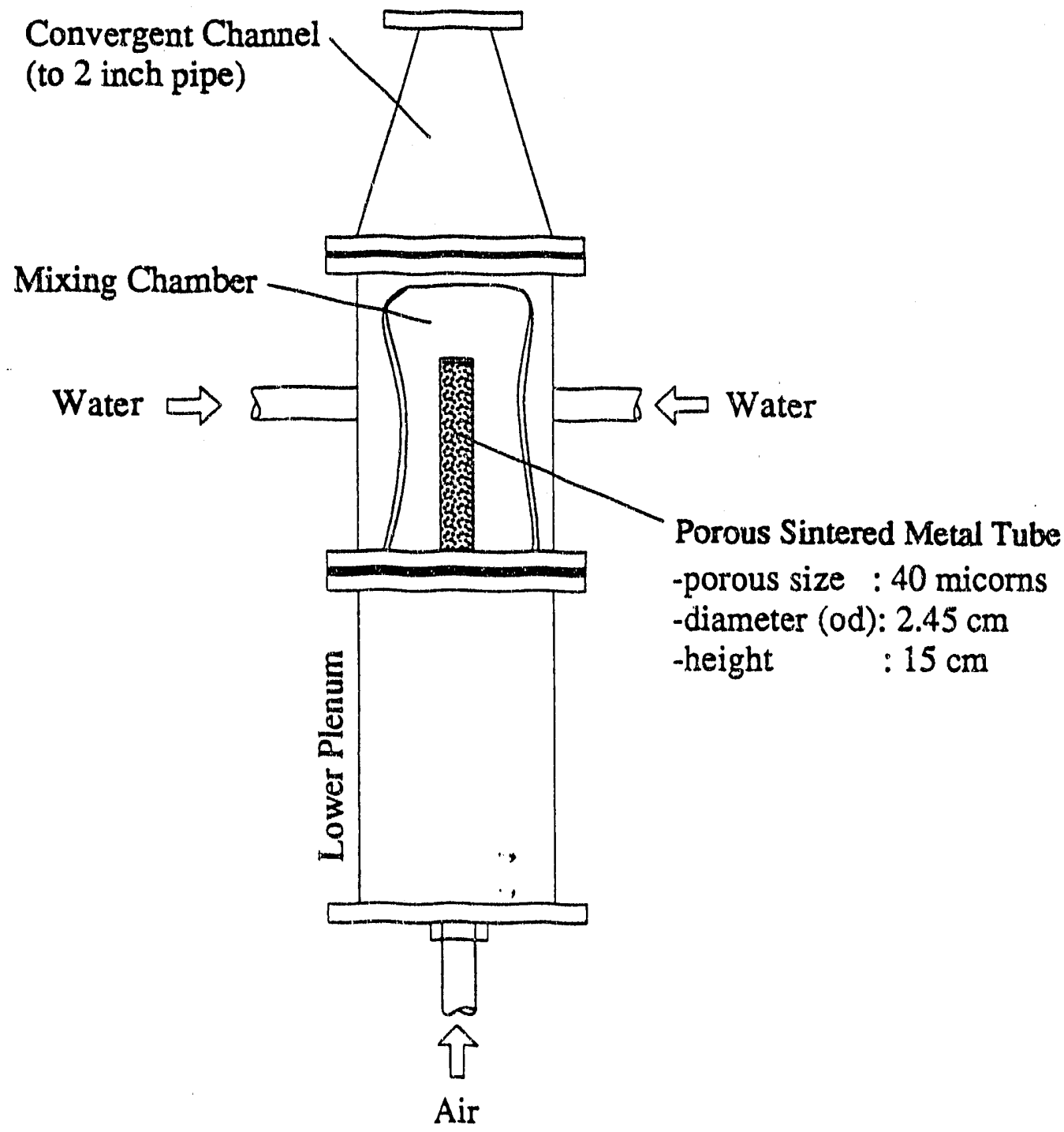

Fig. 11 The Bubble Generator Design Schematic 
together in the mixing house, the air-water two-phase mixture flows upward through a short convergent section into the test section. This type of bubble generator does not give a single bubble size but a size distribution. From the experimental results, it shows that the average bubble diameter does not depend on the liquid flow rate except at the very low superficial liquid flow $\left(j_{l}=10 \mathrm{~cm} / \mathrm{s}\right)$. The average bubble diameter measured is roughly $3.8 \mathrm{~mm}$. and the average diameter increases slightly as void fraction increases. In the $j_{l}=10 \mathrm{~cm} / \mathrm{s}$ case, the average bubble diameter changes quite drastically to 5 to $6 \mathrm{~mm}$. The reason for the change is not yet known since the exact bubble generation mechanism is not completely understood. It may well be due to the bubbles coalescing at the entrance region. However in this report, the knowledge of exact bubble character is not needed.

\subsection{Flow Rate Measurements}

\subsubsection{Superficial Gas Velocity}

The average air flow rate is measured by a flow meter (see Fig.10). The volumetric flow rate can be read directly from the flow meter and the superficial gas velocity is calculated by:

$j_{8}=\frac{Q_{8}}{A}$

where $Q_{g}$ is the volumetric flow rate read from the flow meter and $A$ is the cross-section area of the pipe. Since the gas flow meter is calibrated at a certain pressure, which may be different from the experimental pressure condition, the actual flow rate must be corrected from the meter's value. The gas is injected into the system from the pressurized the tank, the injection pressure is roughly $7.8 \mathrm{psig}$ in all our experiments. The correction relation is given by:

$Q_{g}=Q_{g m} \sqrt{\frac{P_{i n j}+P_{c a l}}{P_{c a l}}}$

where $Q_{\text {on }}$ is the gas flow rate read from the meter, $P_{\text {cal }}$ is the pressure where the flow meter is calibrated, and $P_{\text {inj }}$ is the injection pressure where the flow meter is used. Since gas is a compressible fluid, the pressure change will cause the gas volume change roughly according 
to the prefect gas law in a low pressure system. And the pressure head of the $3.75 \mathrm{~m}$ height two-phase mixture column is significant from the bottom to the top of the test section. The local volumetric flow rate is then calculated by:

$\mathrm{Q}_{B}(h)=\mathrm{Q}_{B} \frac{\mathrm{P}_{c a l}+\mathrm{P}_{i n j}}{\mathrm{P}_{c a l}+\rho_{m} g h}$

where $h$ is the distance from the top plenum to the probe local, and $\rho_{m}$ is the average two-phase mixture density. In most of the plots the superficial gas flow rate is given in local value that is calculated from Eq.(46).

\subsubsection{Superficial liquid flow rate}

The liquid volumetric flow rate is measured by the pressure drop across an orifice (see Fig.10). The pressure drop $\Delta P_{\operatorname{man}}$ is measured by a manometer using a fluid with specific gravity equal to 2.95 . The equation of conversion is:

$j_{l}=\frac{C_{D} A_{o}}{\rho A} \sqrt{\frac{2 \rho \Delta P_{\operatorname{man}}}{1-\gamma^{4}}}$

where $A_{0}, C_{D}, \gamma$, and $\rho$ are the orifice area, frictional coefficient, area ratio and density of the fluid (in our case is water) respectively. The orifice size that we used is $A_{0}=4.256 \times 10^{-4}$ $\mathrm{m}^{2} . \mathrm{C}_{\mathrm{D}}$ is calibrated by experiment. $\gamma$ is the area ratio between the orifice and the pipe size.

\section{Experiment Results and Remarks}

\subsection{The Experiments}

The local measurement is done by a double sensor probe that is described in section 5.3.2 and 5.3.3. The probe is inserted into two axial location at $L / D=8$ and $L / D=60$. The first location represents the developing zone and the second location represents the developed zone. Local parameters such as void fraction, interfacial area concentration, sauter mean diameter, bubble frequency, and bubble velocity are recorded by the local probe measurement. The double probe is mounted on a micrometer traveller which gives the reading of the radial location. Roughly, 12 to 16 radial locations are scanned on each set of measurements. The radial distance is $1 / 10$ of an inch at $r / R<0.7$. When the probe is moved 
closer to the wall the radial distance between scans is roughly $1 / 40$ to $1 / 20$ of an inch. This is because we know that the local void profile changes more rapidly at the near wall range. The probe signal is digitized and processed by a computer program. The signal processing subroutines are implemented by the algorithm given by section 4 . Four different liquid flow rates are measured in combination with four different gas injection rates and they are listed as follows:

1) The superficial liquid flow rates

$\mathrm{j}_{\mathrm{i}}=1.0,0.6,0.4$, and $0.1 \mathrm{~m} / \mathrm{s}$

2) The superficial gas flow rates

$j_{g}=0.0965,0.0696,0.0384$, and $0.0192 \mathrm{~m} / \mathrm{s}$.

One must notice that the superficial gas injection is the flow rate read at the injection point while in most of the plots the superficial gas flow rates are given at the specific axial location. At the same time, the pressure drop across an axial distance is measured before each experiment. Using the homogeneous model, the average void fraction can be computed from the pressure drop data. The agreement is very good. Then area average void fraction is computed from the local void fraction that is measured by the probe. Thus the local void fraction measurement is checked with the global measurements in order to ensure that the probe measurement is correct (See Fig 12.a and 12.b).

\subsection{Local Data Presentation}

There are a total of 16 sets of data from each axial location. First the local data is grouped by their superficial liquid velocity and presented in Fig 13 to Fig 20 and four different gas flow rates are given as the independent parameters. The radial profile of void fraction, interfacial area concentration, sauter mean diameter, bubble frequency and the bubble velocity are plotted in different graphs (from a to e respectively). The open symbols are assigned to the data points taken from the entrance region $(L / D=8)$ while the filled symbols are assigned to the data points taken from the developed region $(\alpha / D=60)$. We keep the same symbol for the same liquid flow rate all along. This set of plots shows the effect of the gas flow on the radial phasic profile with a constant liquid flow rate.

Secondly, the same sets of data are grouped by their superficial gas velocity as presented in Fig 21 to 24 . Only two superficial gas flow rates are picked $j_{s}=0.0965$ and 0.0192 $\mathrm{m} / \mathrm{s}$ ) and all four superficial liquid flow rates are given as the independent parameters. The 

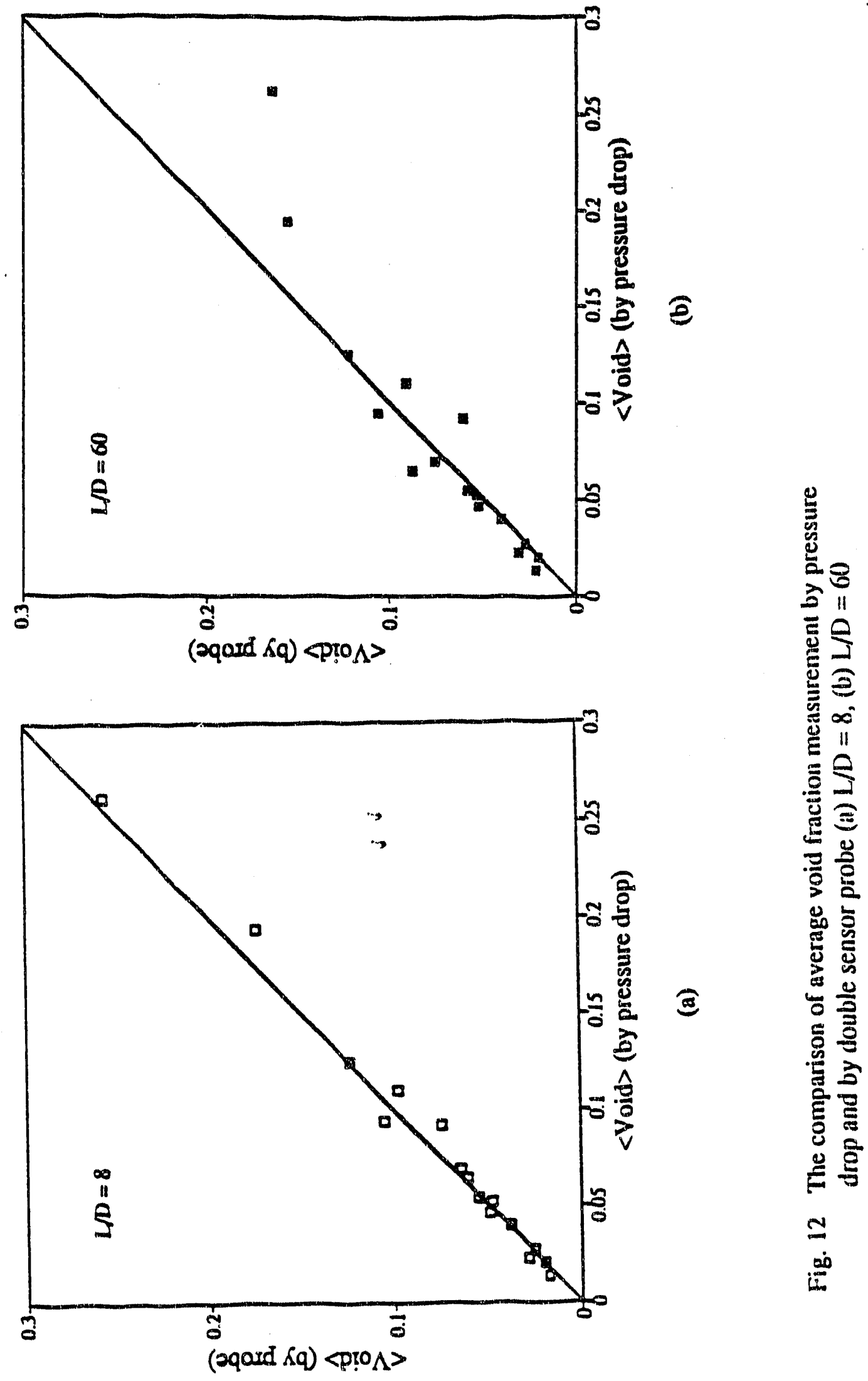
same five local parameters are plotted and the same symbol assignments as in the first set of plots are made. In this set of plots, the liquid flow effect on the radial phasic profile is shown while the same amount of the gas are injected into the liquid.

The last set of the local parameter plots is the comparison of the radial profile between the $L / D=8$ and the $L / D=60$ (See Fig.25 to.44). The radial profile of the same five local parameters are plotted. This comparison shows how the bubbles redistribute themselves and how their sizes change in the developed region.

\subsection{Observations}

\subsubsection{Constant Liquid Velocity}

The local profiles behave very much alike for the $\mathrm{j}_{l}=1.0,0.6$ and $0.4 \mathrm{~m} / \mathrm{s}$ both at the entrance and the developed regions. At the entrance $(L / D=8)$, all the radial void fraction plots show the typical bubbly flow profile--the void fraction peaks at the near wall region. The void peaks at roughly $r / R=0.85$ to 0.90 . Furthermore, a small hump of void peak is also found at the center core. It behaves as if there are two layers of liquid flow and has the boundary at roughly $r / R=0.775$. However, the center hump disappears at the developed region and gives a very flat center core void distribution which agrees with other authors' observations [21 \& 26]. The local interfacial area concentration behaves much the same as the void fraction profile and it also peaks where the void is peaking. This means there is no significant coalescing at the near wall region. This argument is also supported by the plots of bubble frequency. The number of bubhles detected near the wall goes up with the void fraction. It implies that the increase of void fraction is due to the increase of the presence of bubbles. However, the sauter mean diameter profile also goes up from roughly $3.2 \mathrm{~mm}$ at the center core to $4.8 \mathrm{~mm}$ at the near wall region. The diameter ratio is less than two. Then the increase of the sauter mean diameter may be due to the orientation of the ellipsoidal bubble changing from oblate to prolate. Actually, the bubble major axis aligning with the gradient of the velocity field was observed by other authors. The velocity gradient should be steeper at the near wall region. Lastly, the bubble velocity measurement is very close to the theoretical value [3] at the center core. However, it is lower than the prediction at the near wall region and it has a jump at roughly $\mathrm{r} / \mathrm{R}=0.775$. In the $j_{l}=1.0 \mathrm{~cm} / \mathrm{s}$ case, the velocity drops almost $15 \mathrm{~cm} / \mathrm{s}$ from the center line value. There is no obvious explanation 


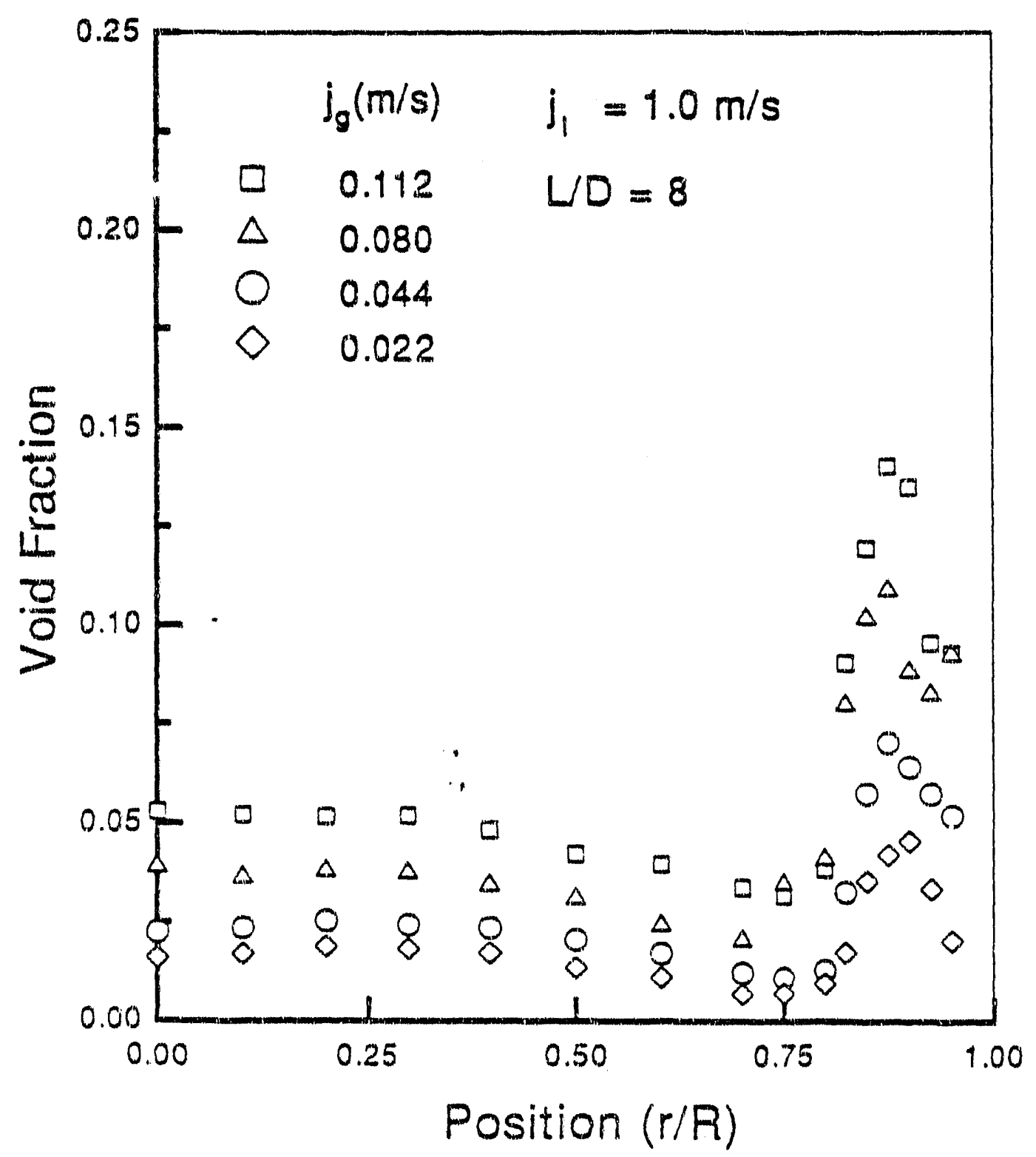

Fig. 13.a. The radial profile of void fraction for air-water bubbly flow at $j_{1}=1.0 \mathrm{~m} / \mathrm{s}$ and $L / D=8$. 

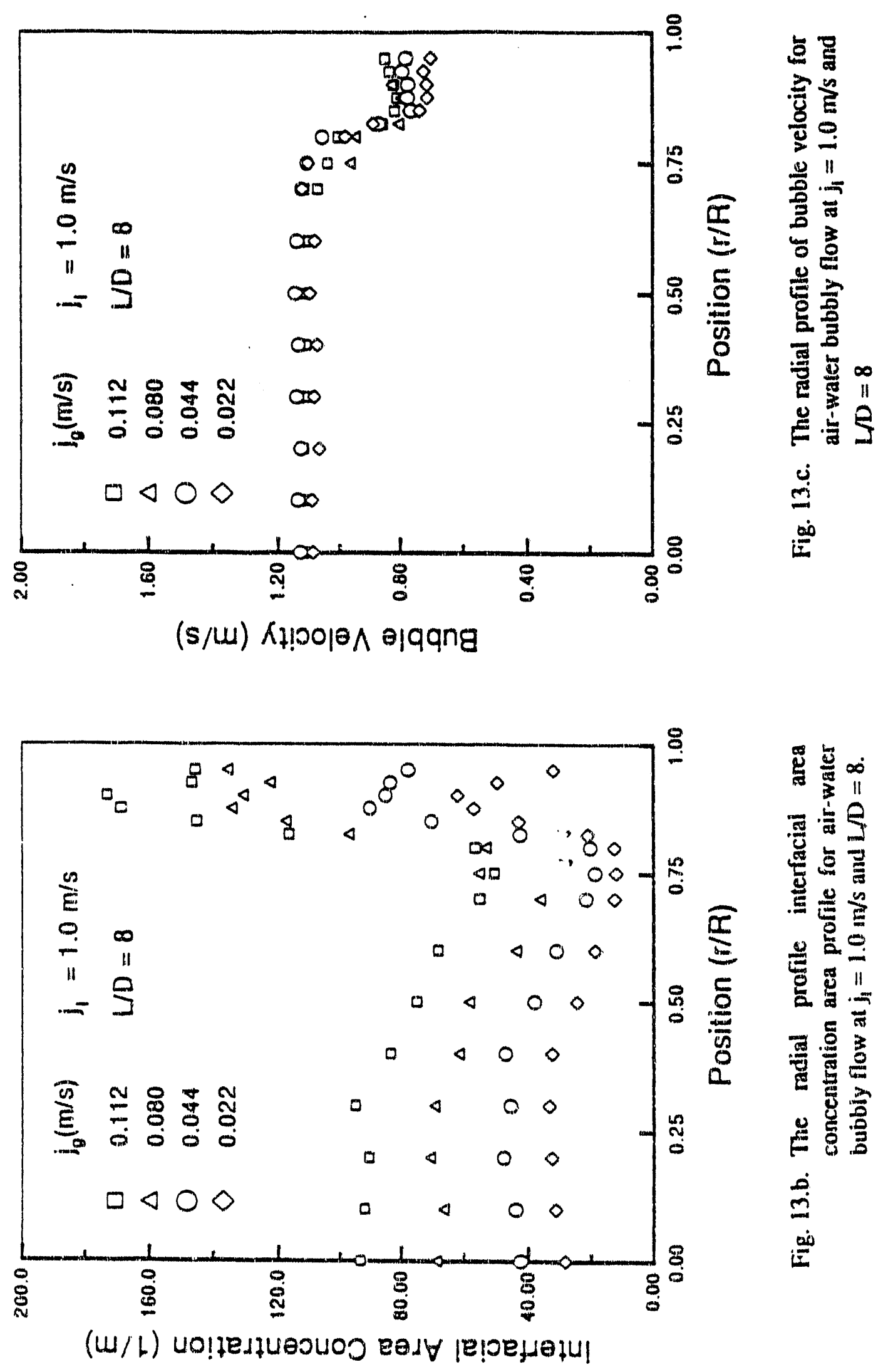

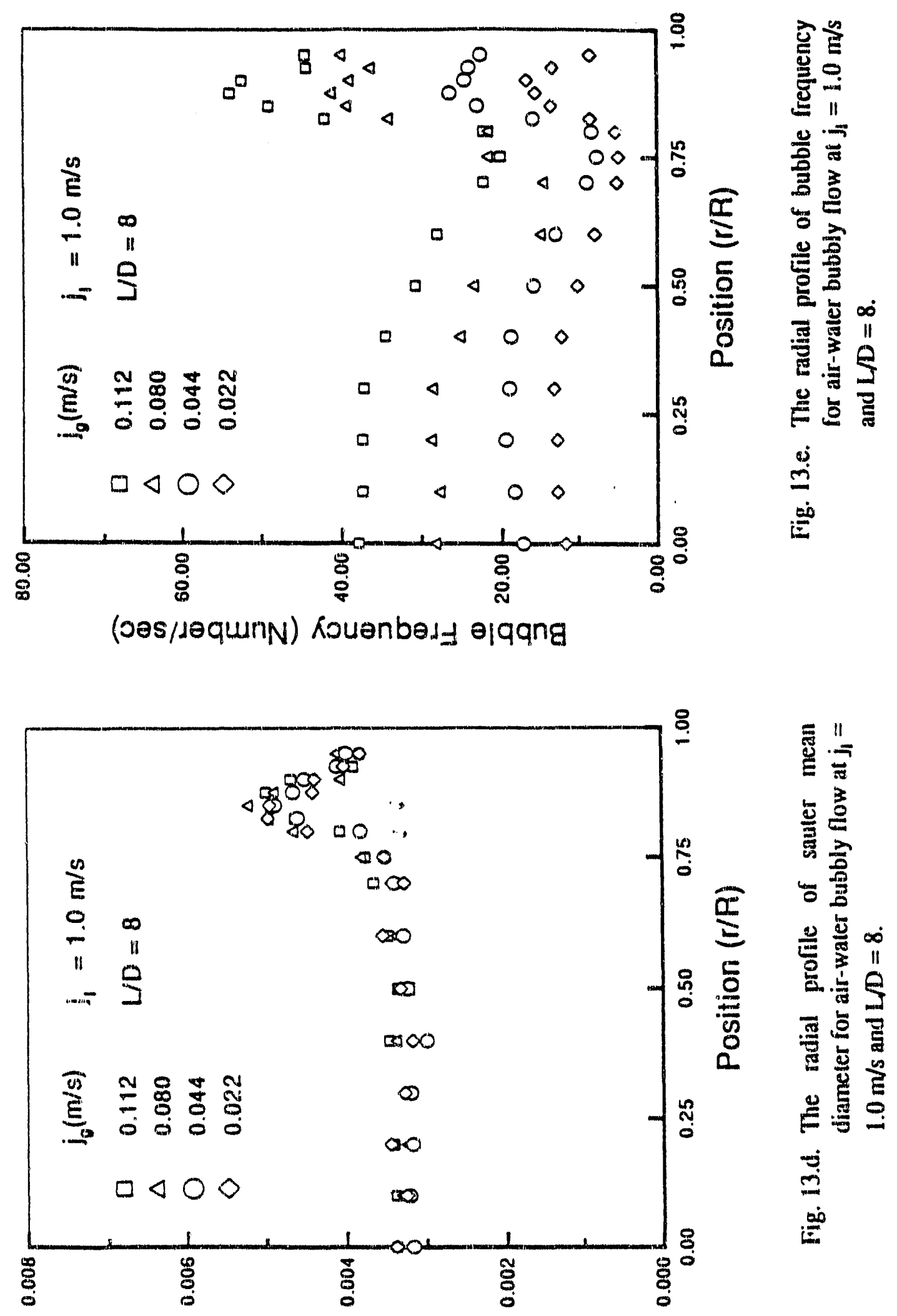

(w) jejaure!g ueew deines 


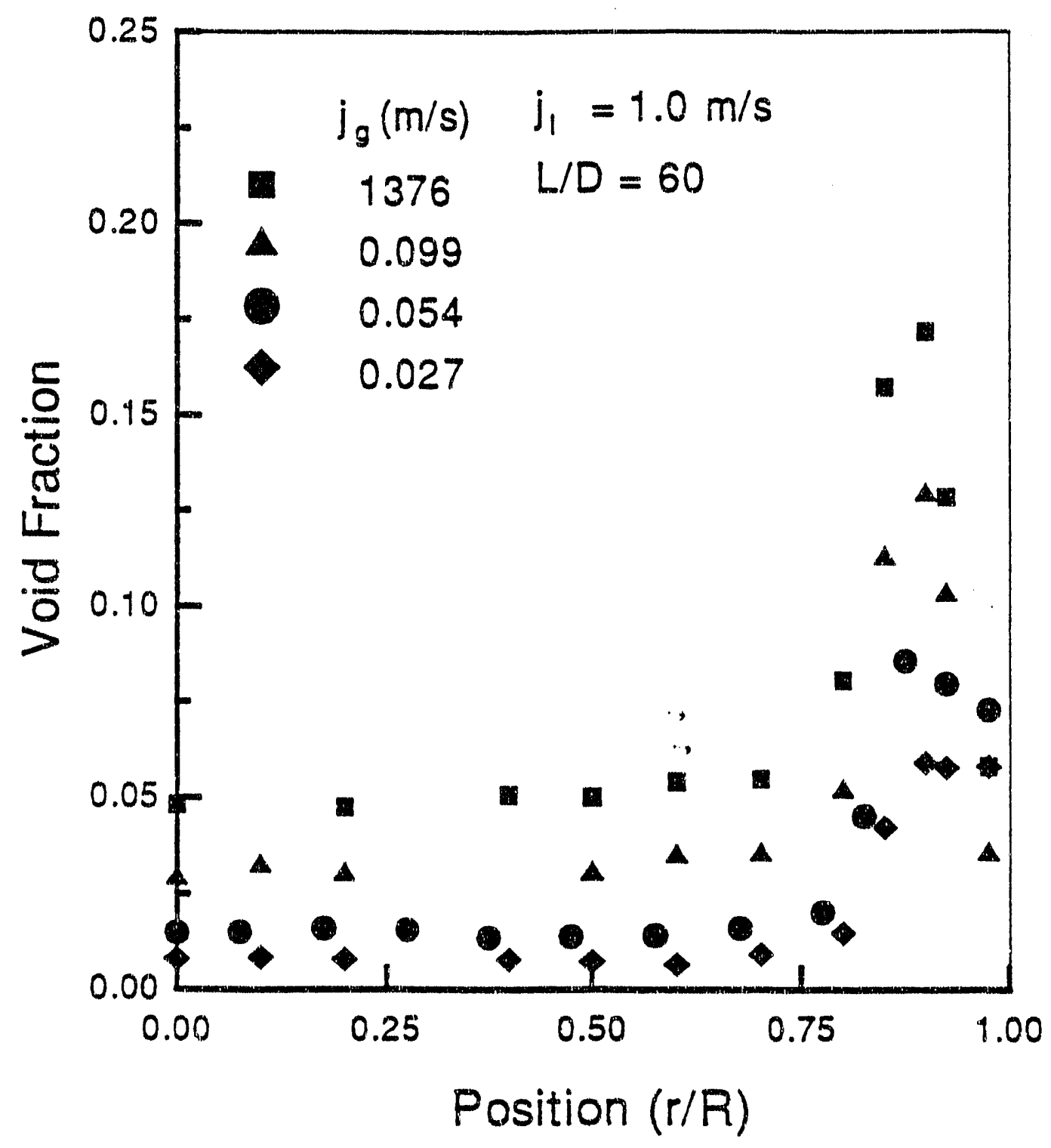

Fig. 14.a. The radial profile of void fraction for air-water bubbly flow at $j_{1}=1.0 \mathrm{~m} / \mathrm{s}$ and $L / D=60$. 

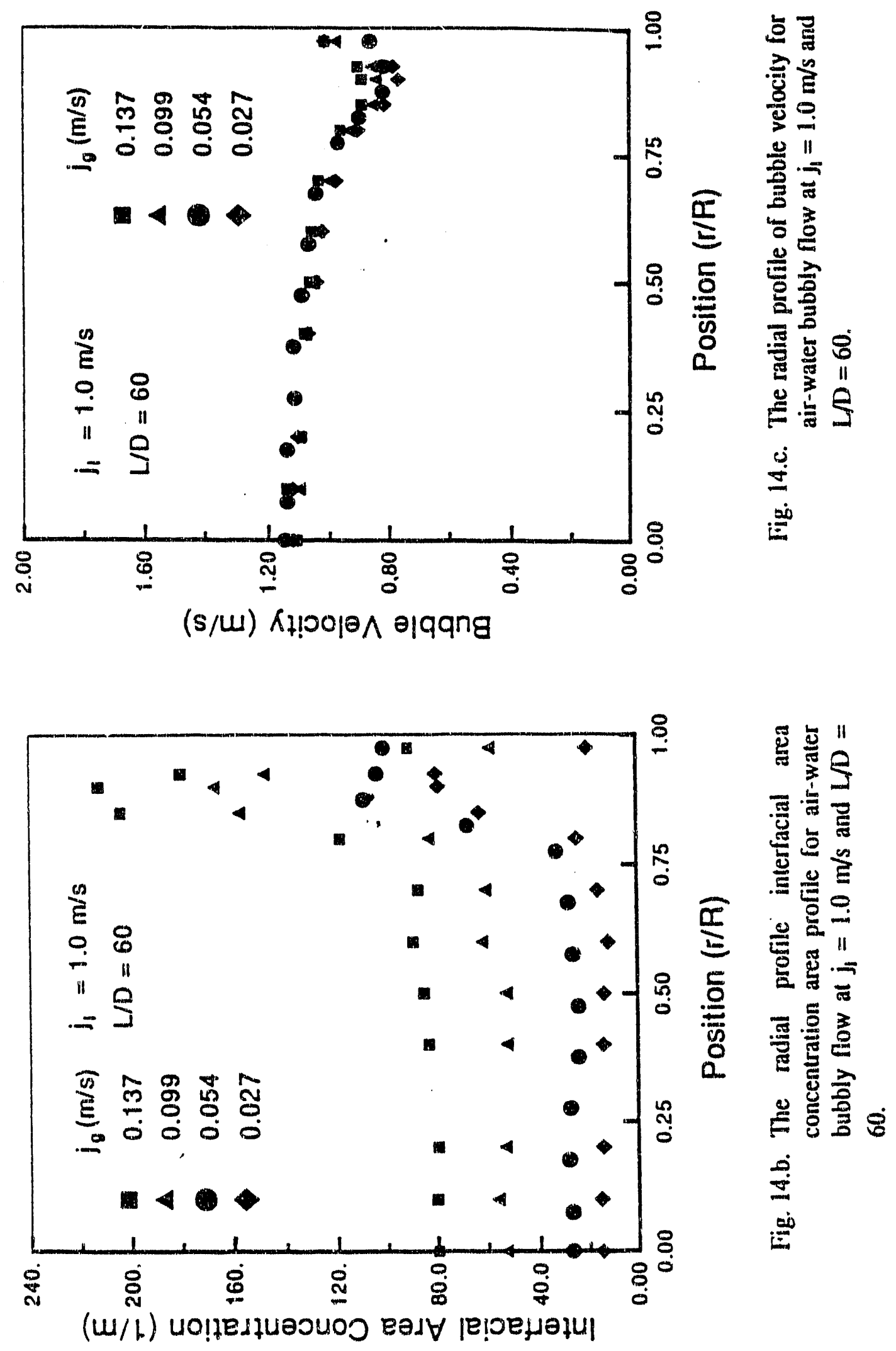

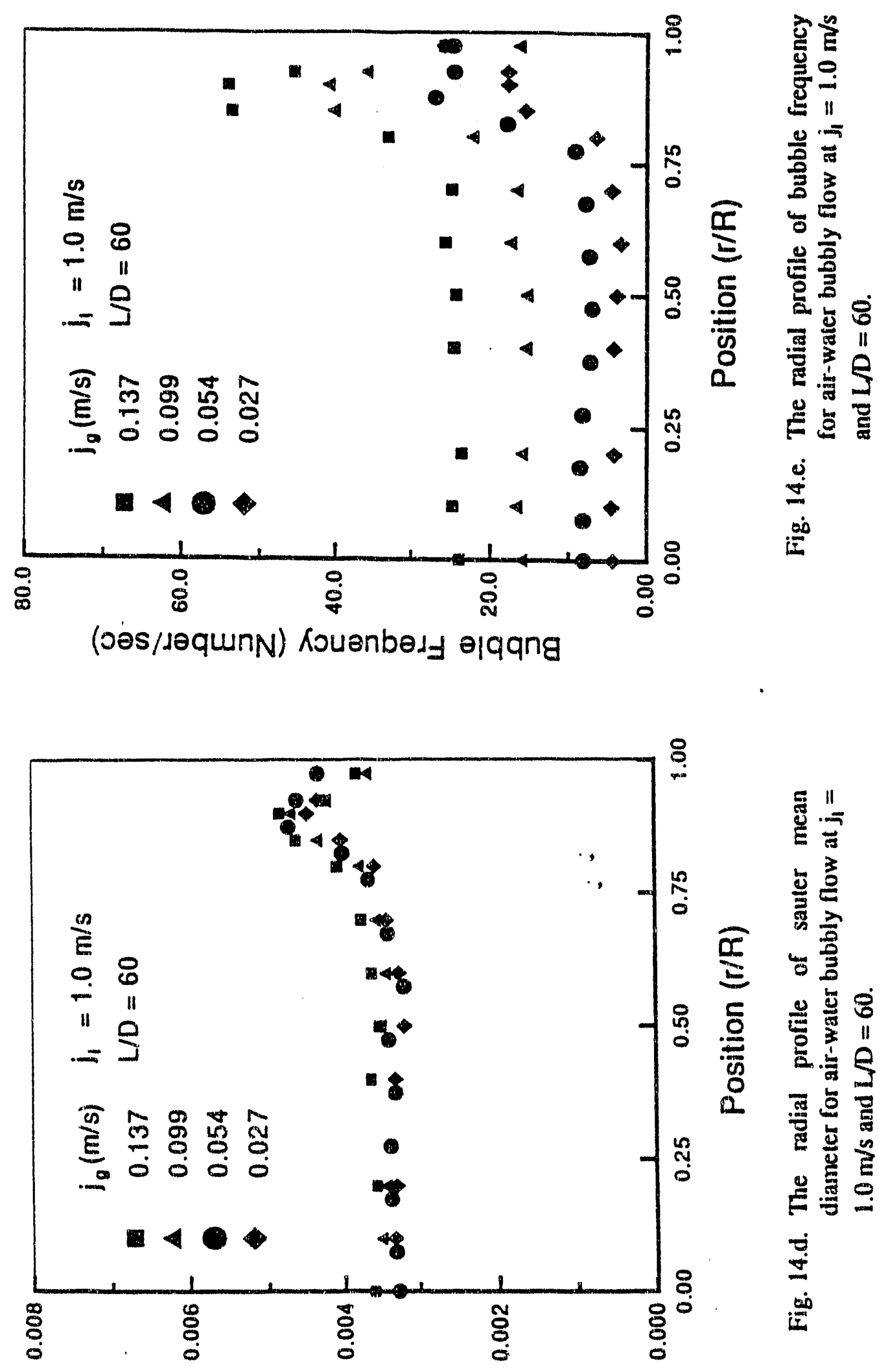

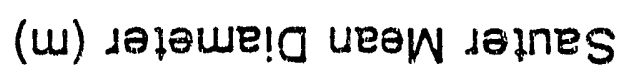




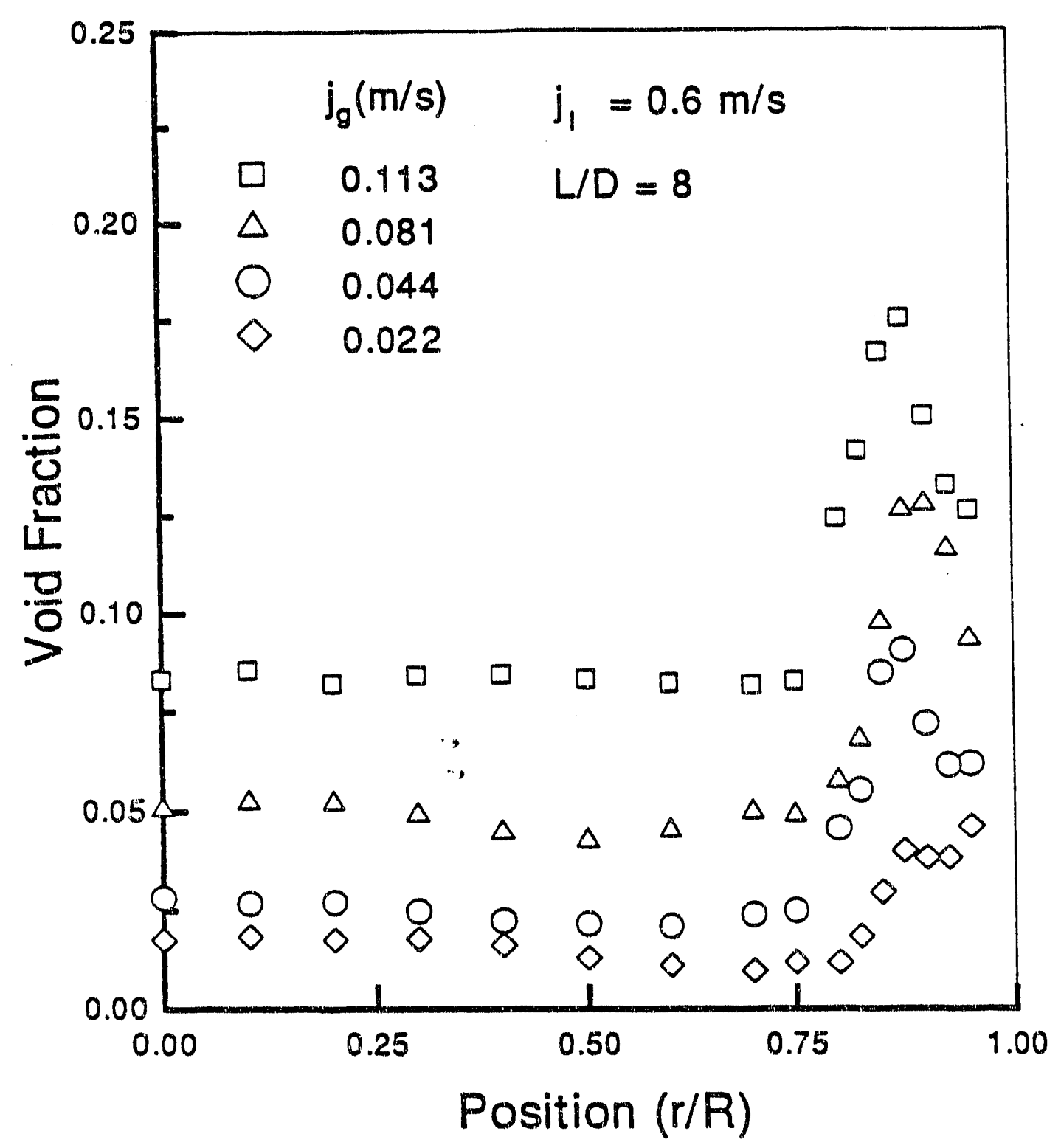

Fig. 15.2 The radial profile of void fraction for air-water bubbly flow at $j_{1}=0.6 \mathrm{~m} / \mathrm{s}$ and $L / D=8$. 

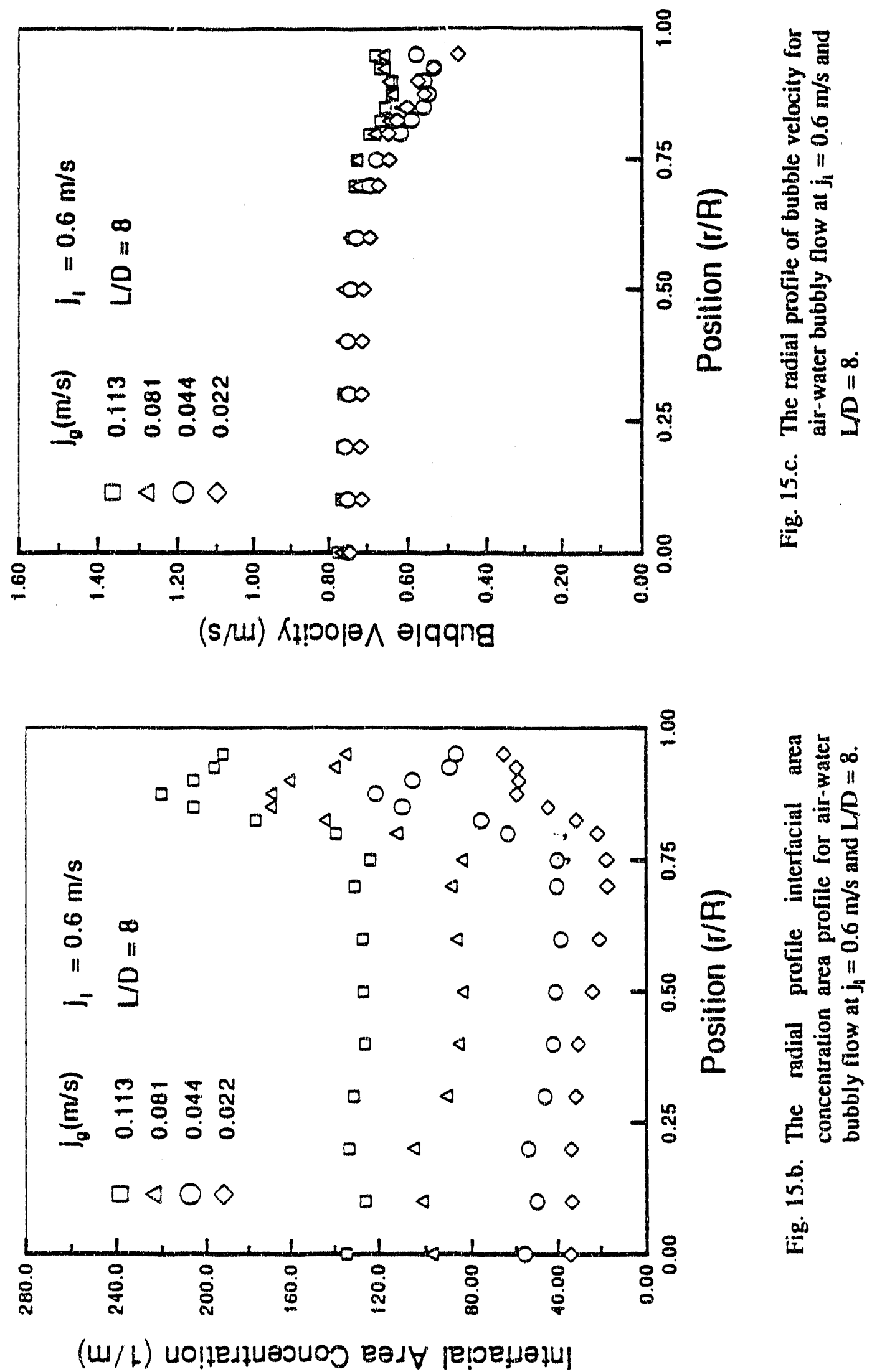

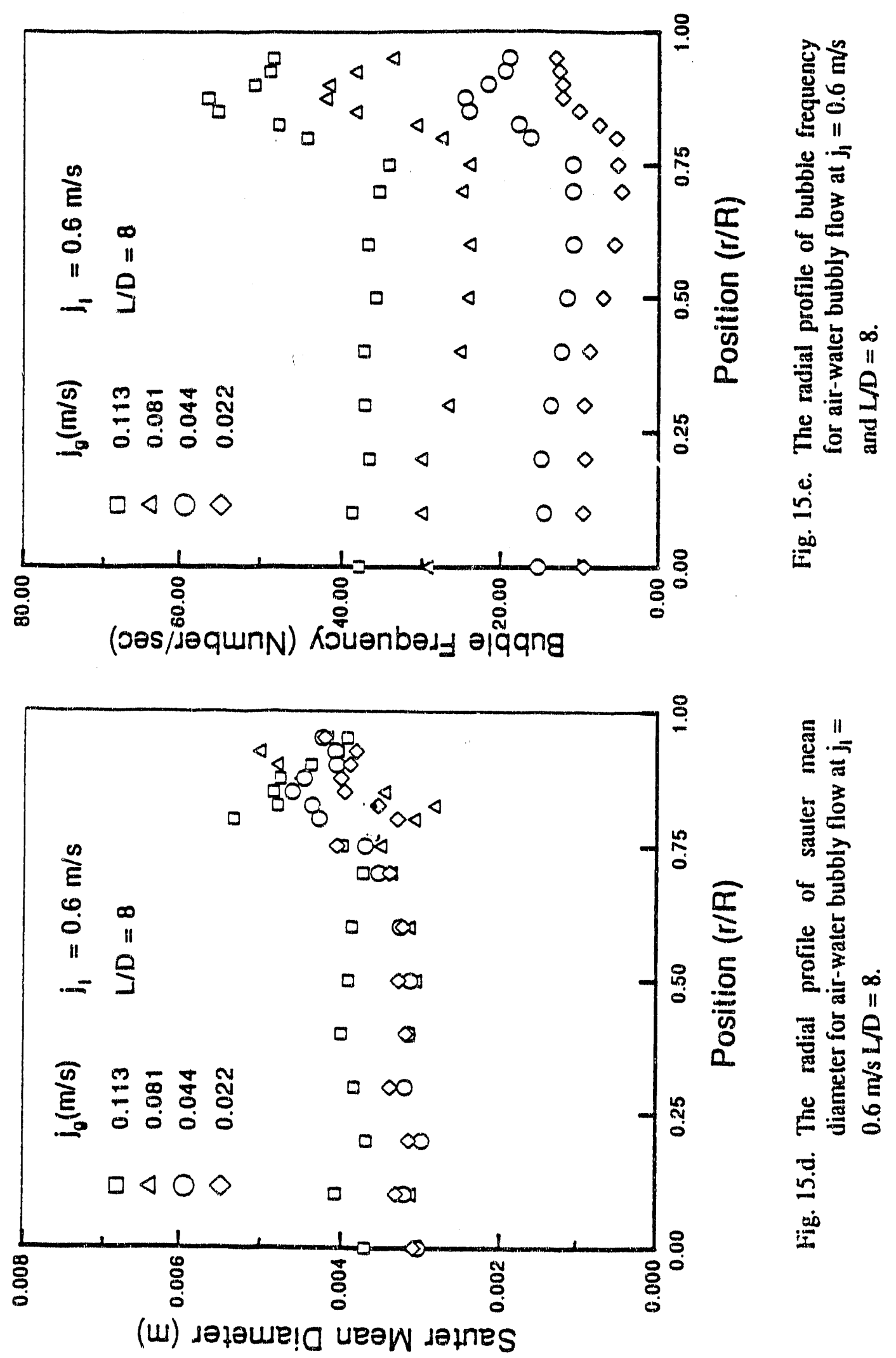


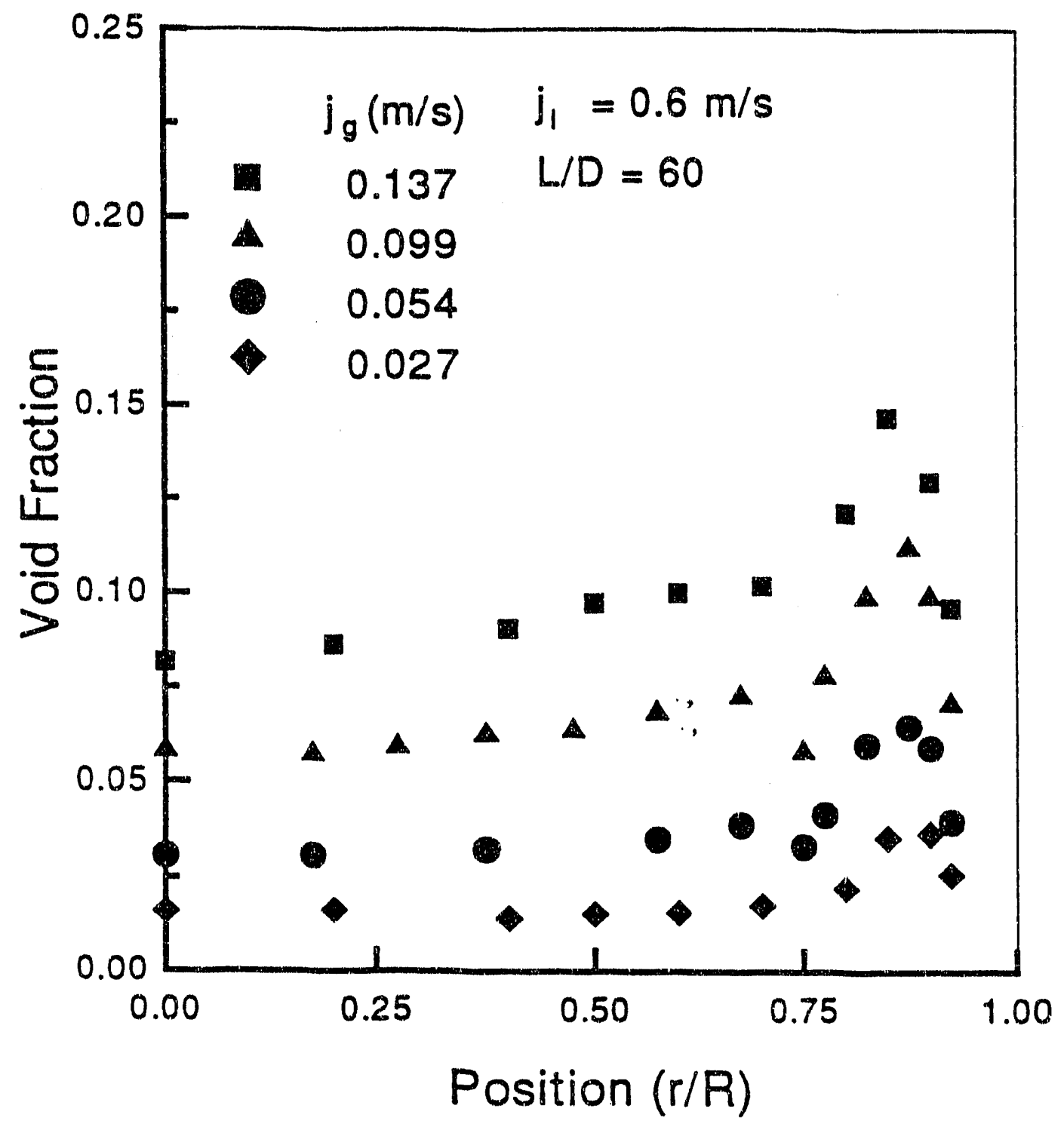

Fig. 16.a. The radial profile of void fraction for air-water bubbly flow at $j_{1}=0.6 \mathrm{~m} / \mathrm{s}$ and $L / D=60$. 

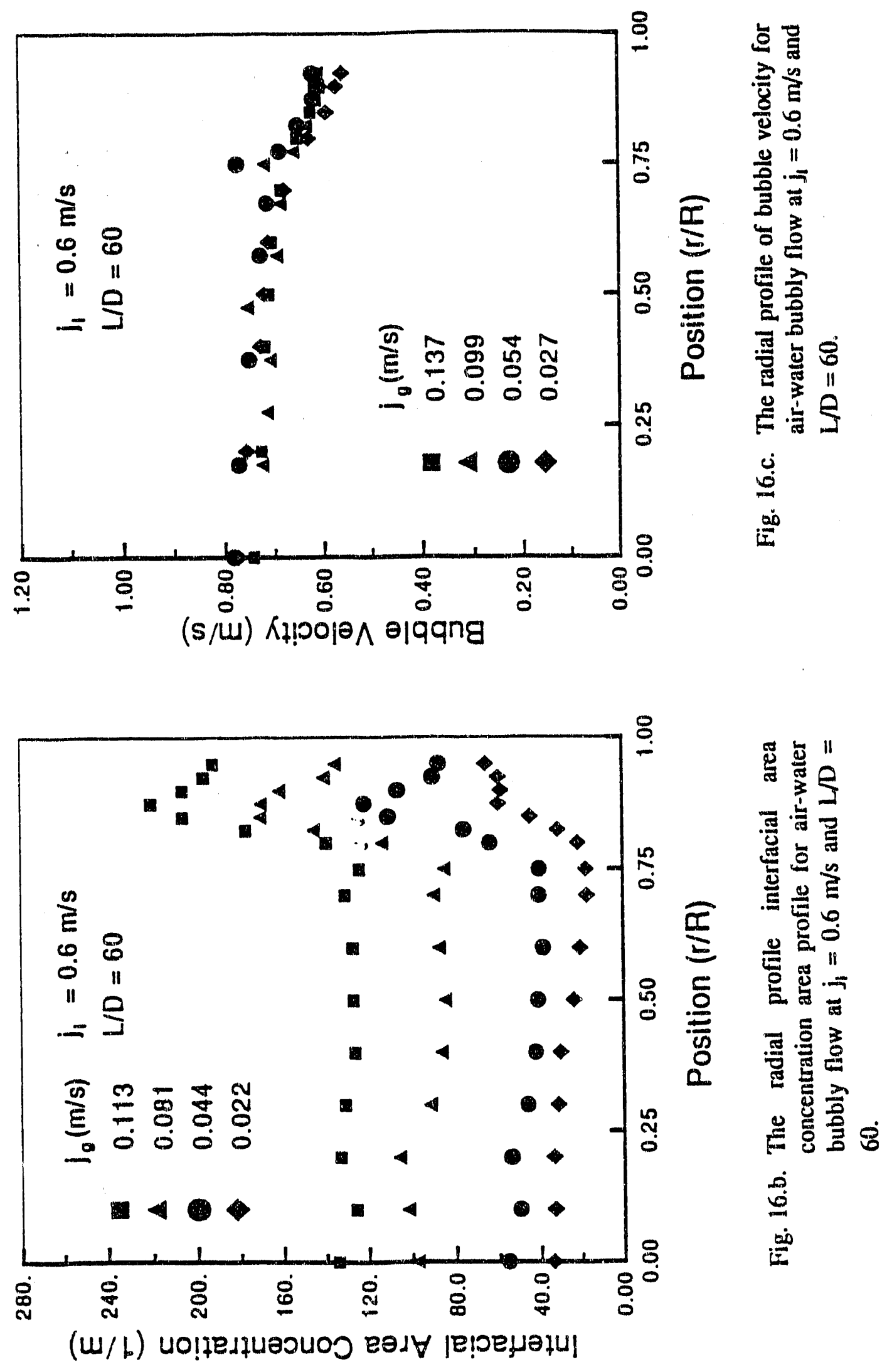

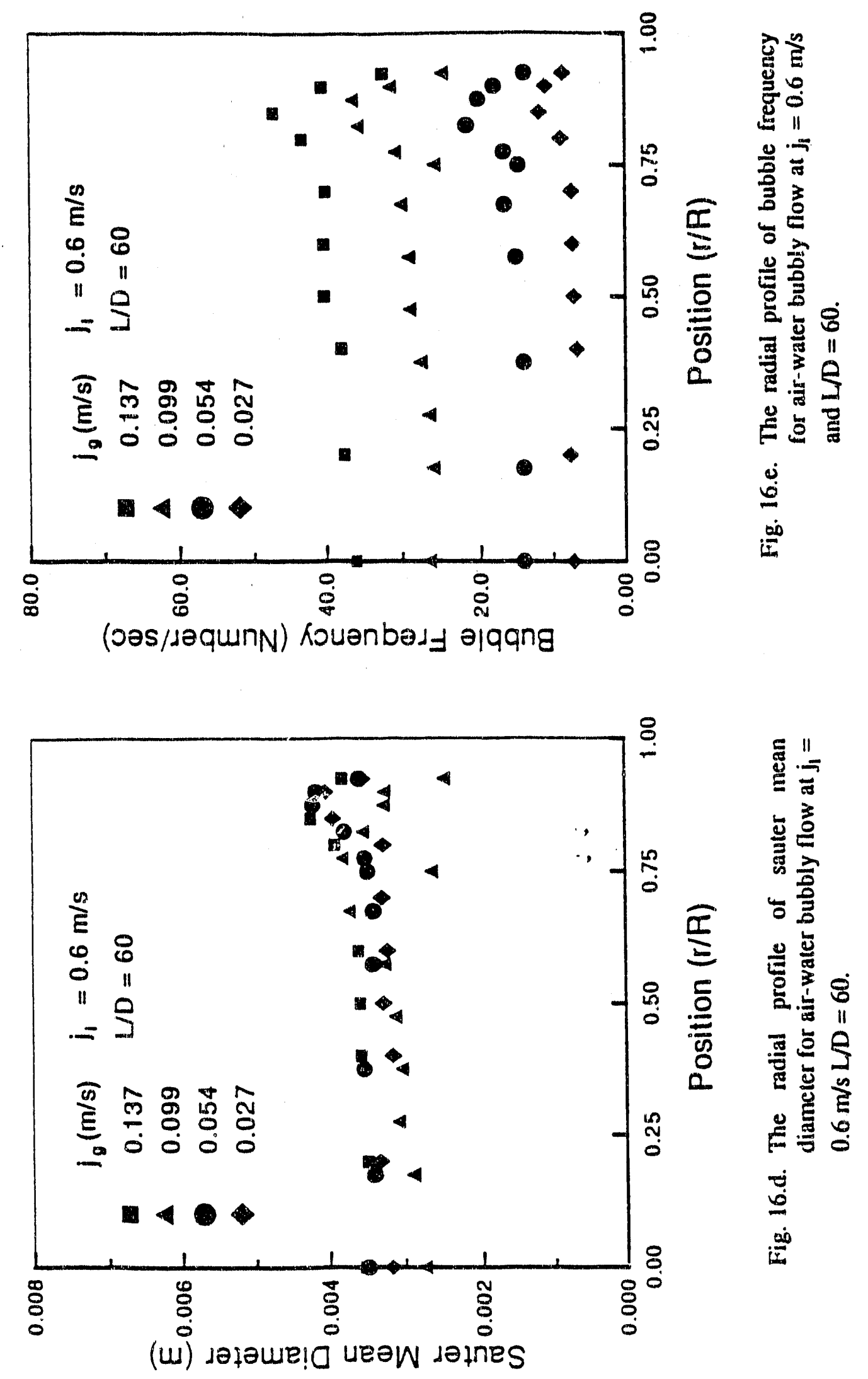


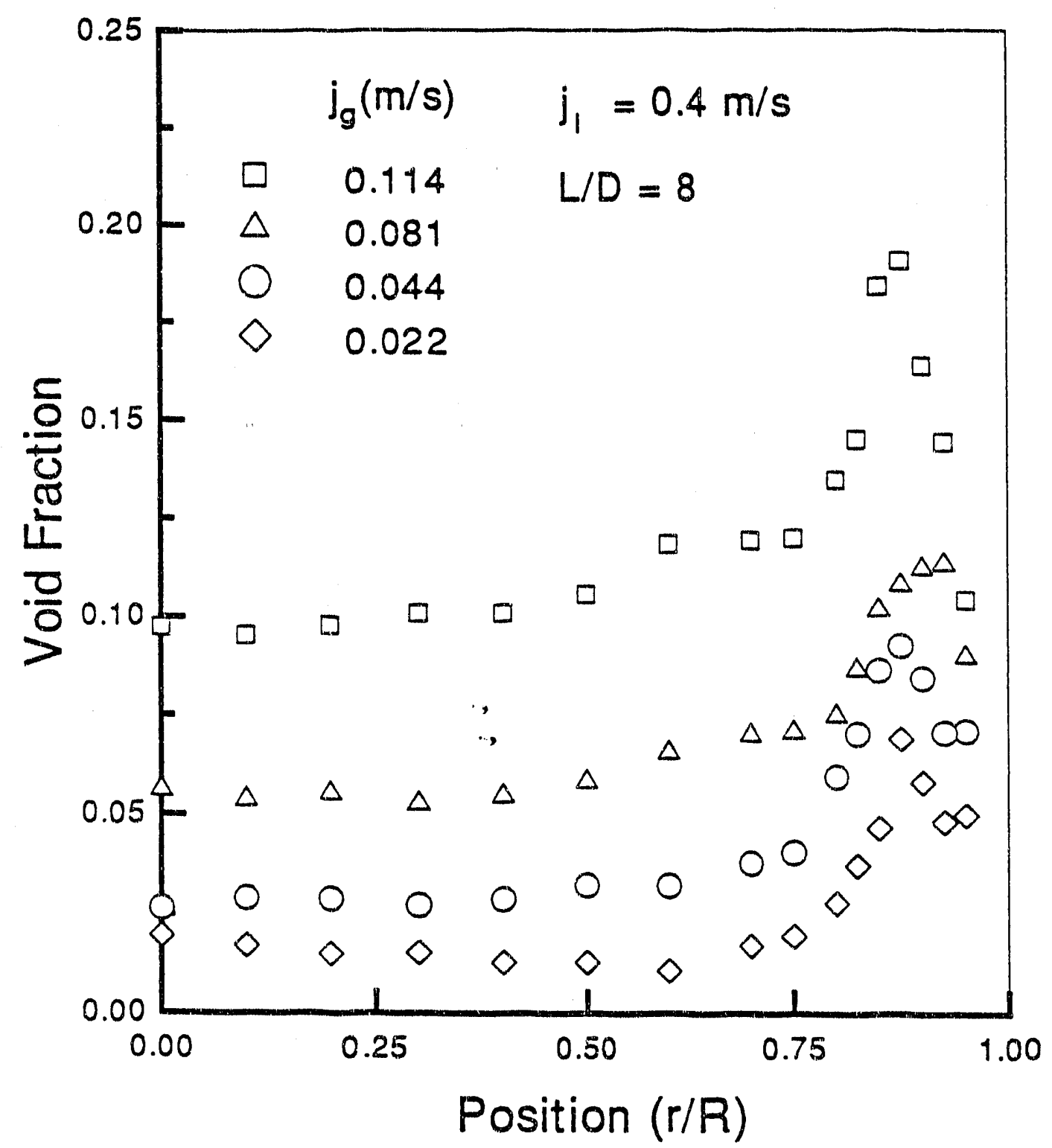

Fig. 17.a. The radial profile of void fraction for air-water bubbly flow at $j_{1}=0.4 \mathrm{~m} / \mathrm{s}$ and $L / D=8$. 

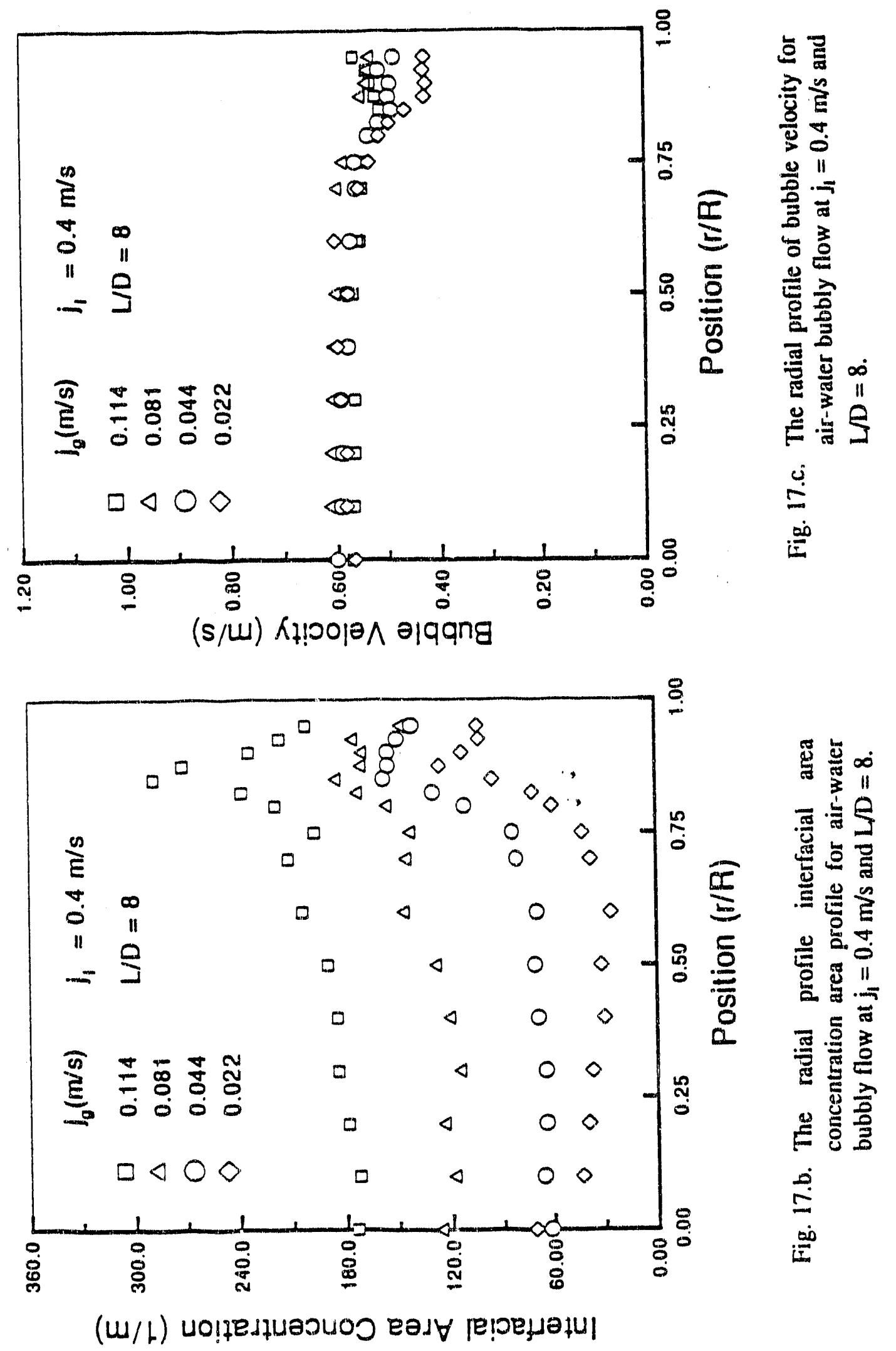

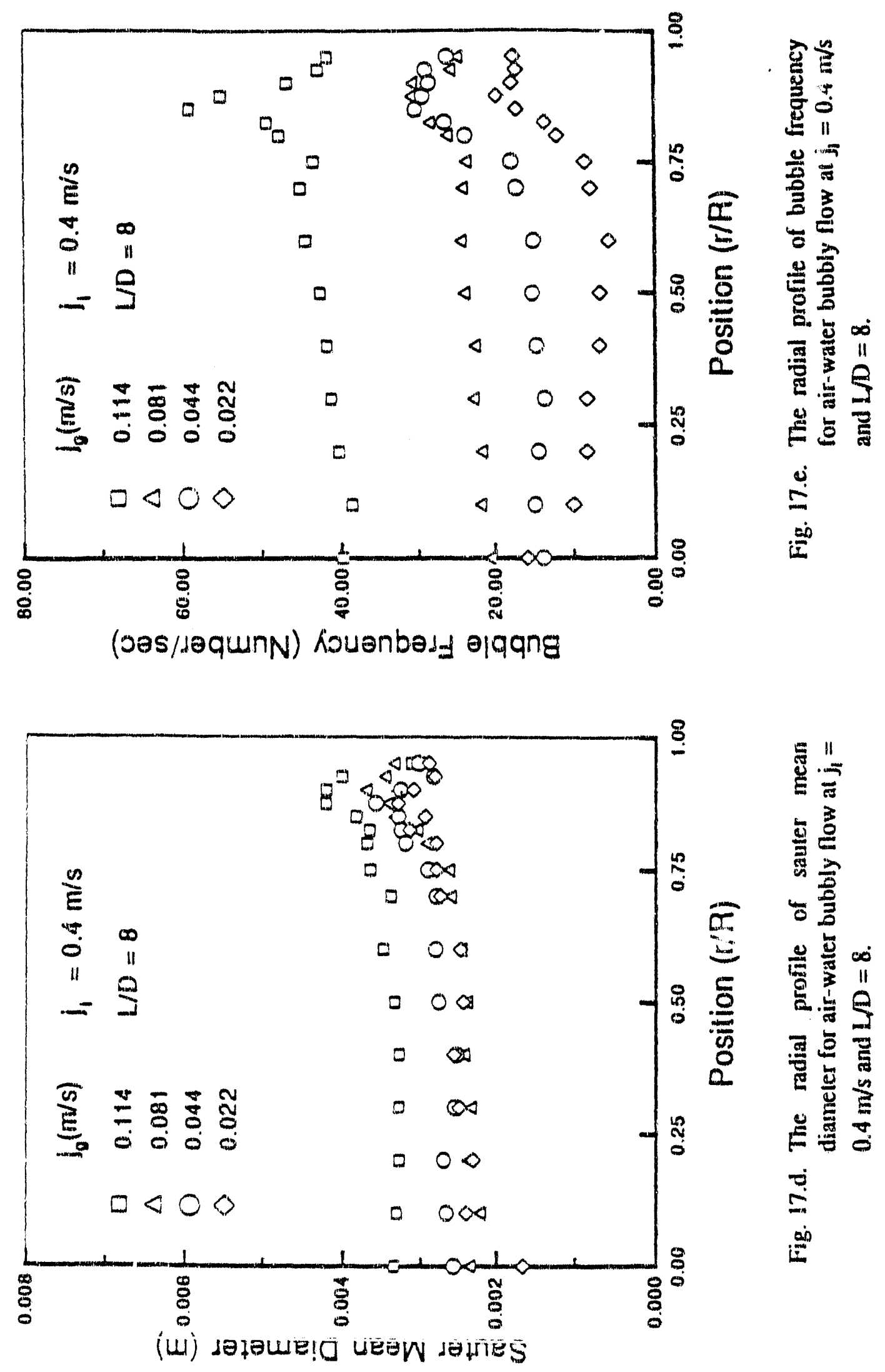


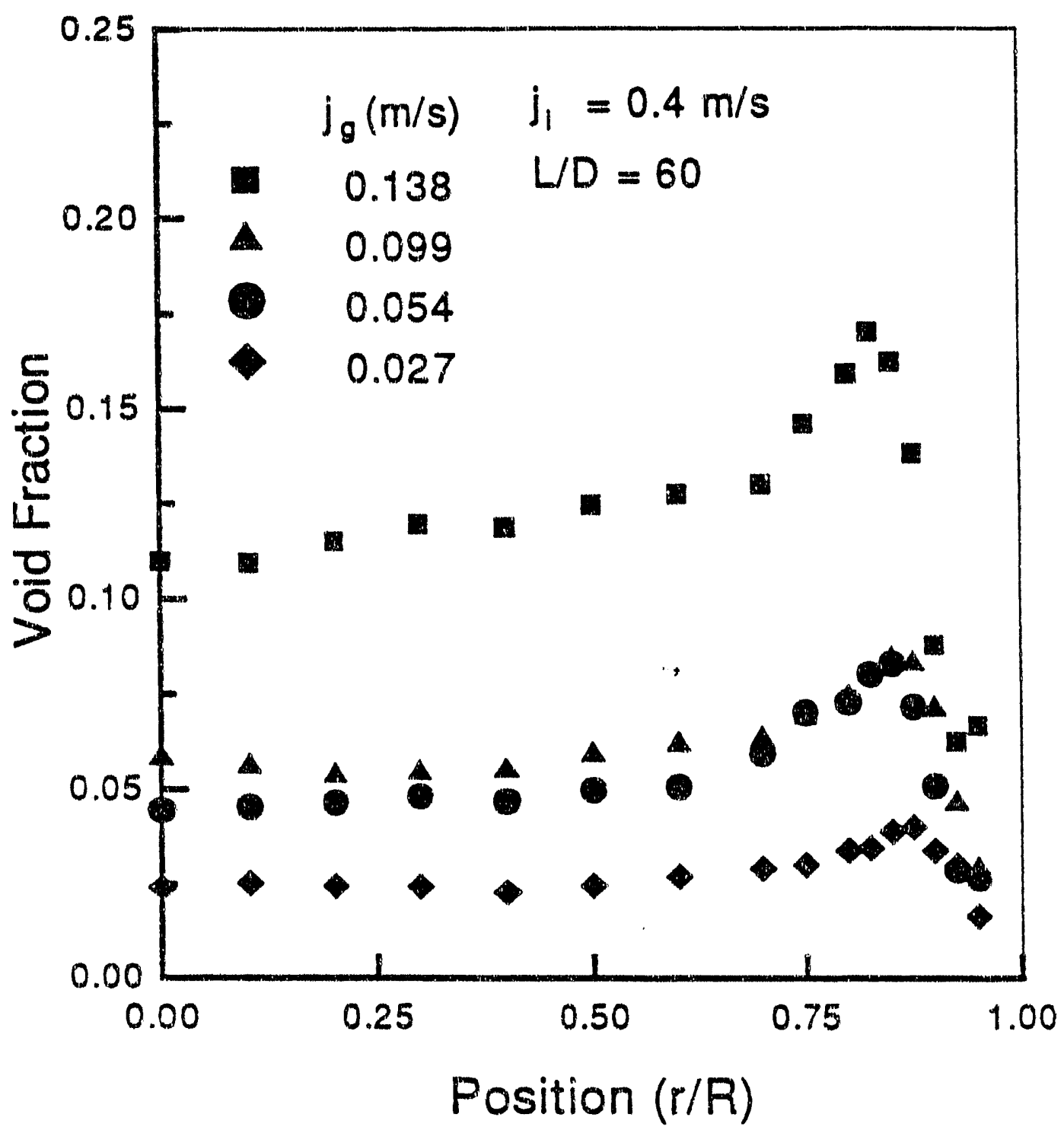

Fig. 18.a. The radial profile of void fraction for air-water bubbly flow ai $j_{1}=0.4 \mathrm{~m} / \mathrm{s}$ and $L D=60$. 

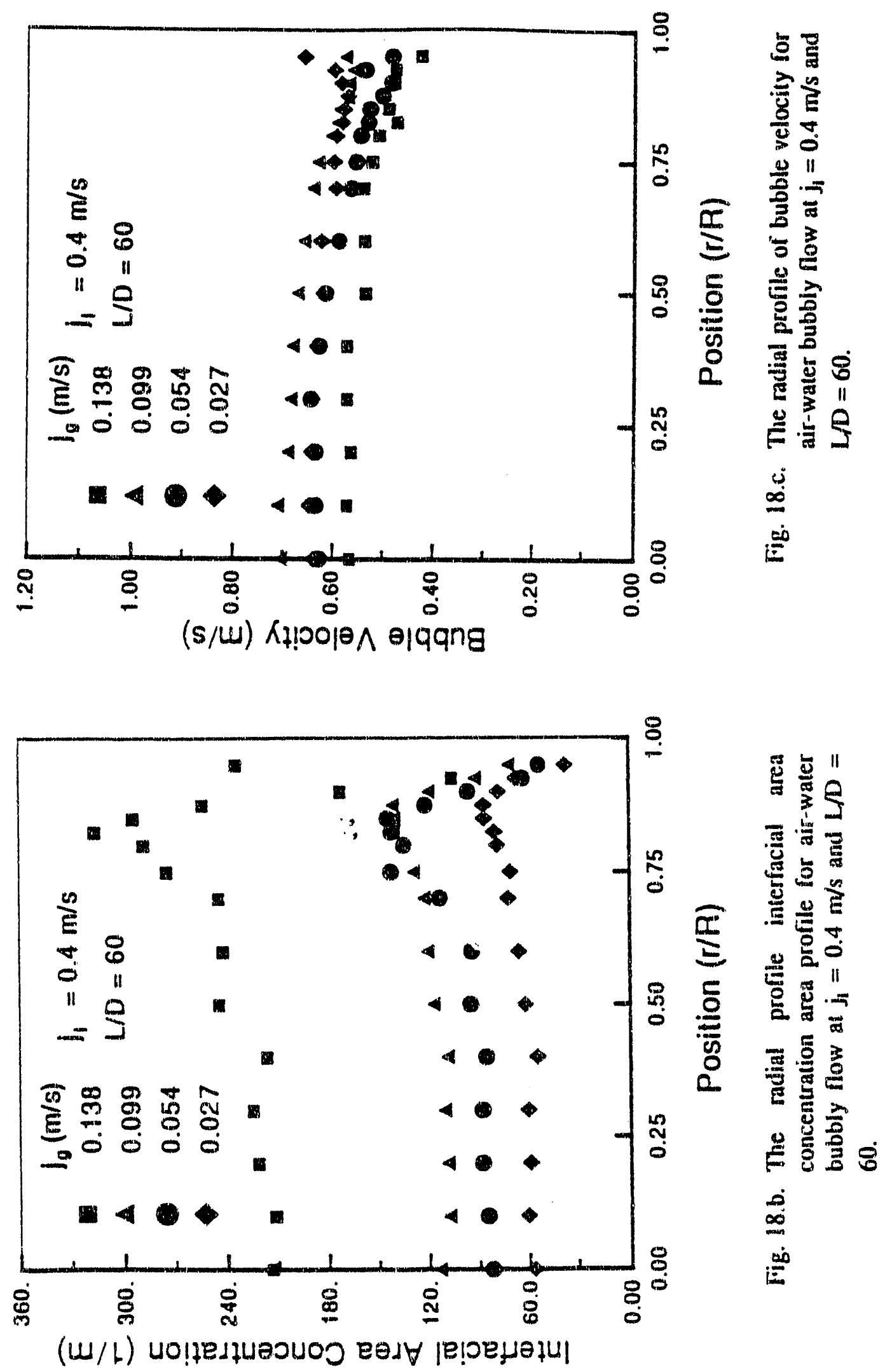

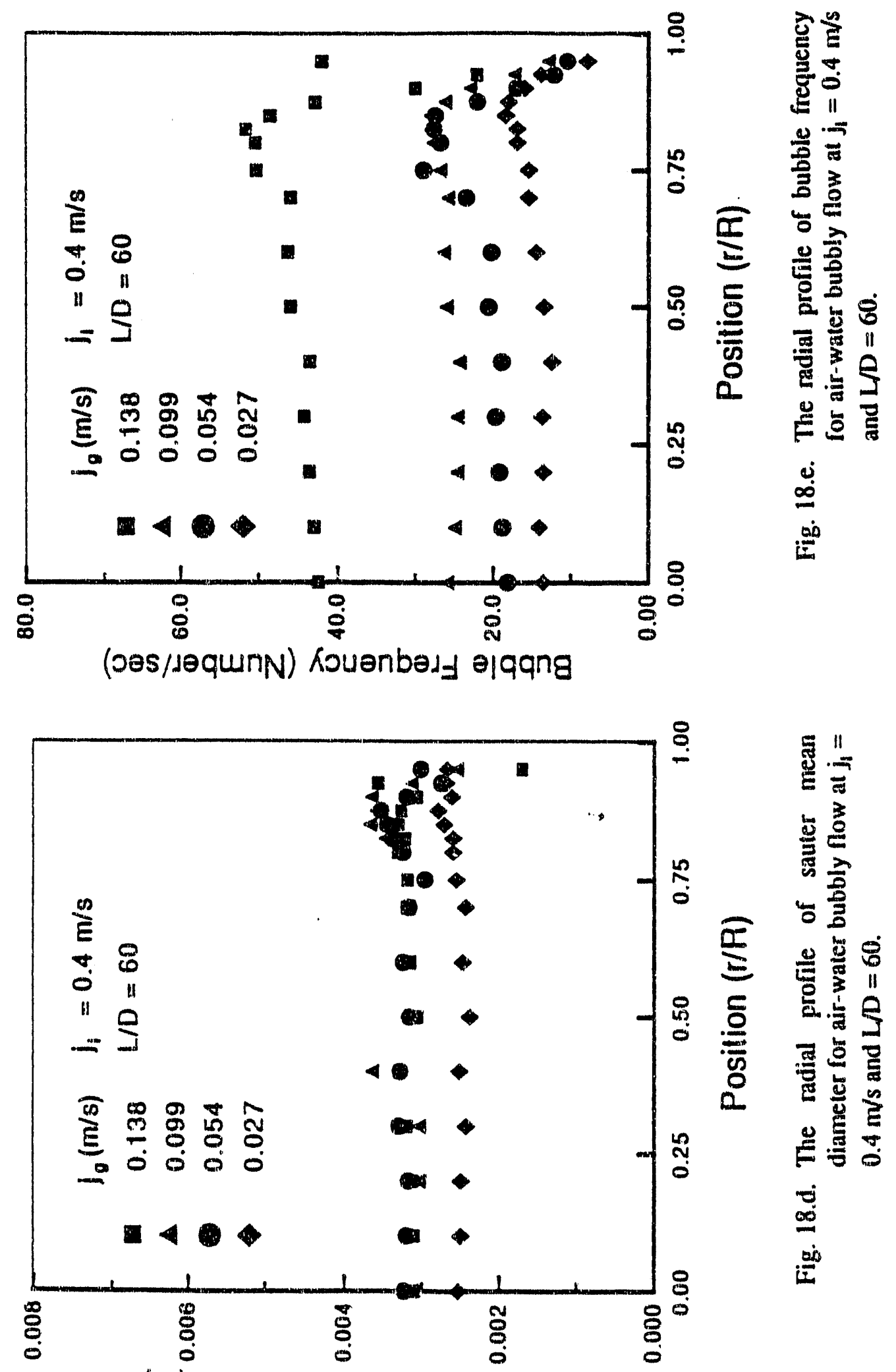

(u) dәjәurio ueaw jafnes 


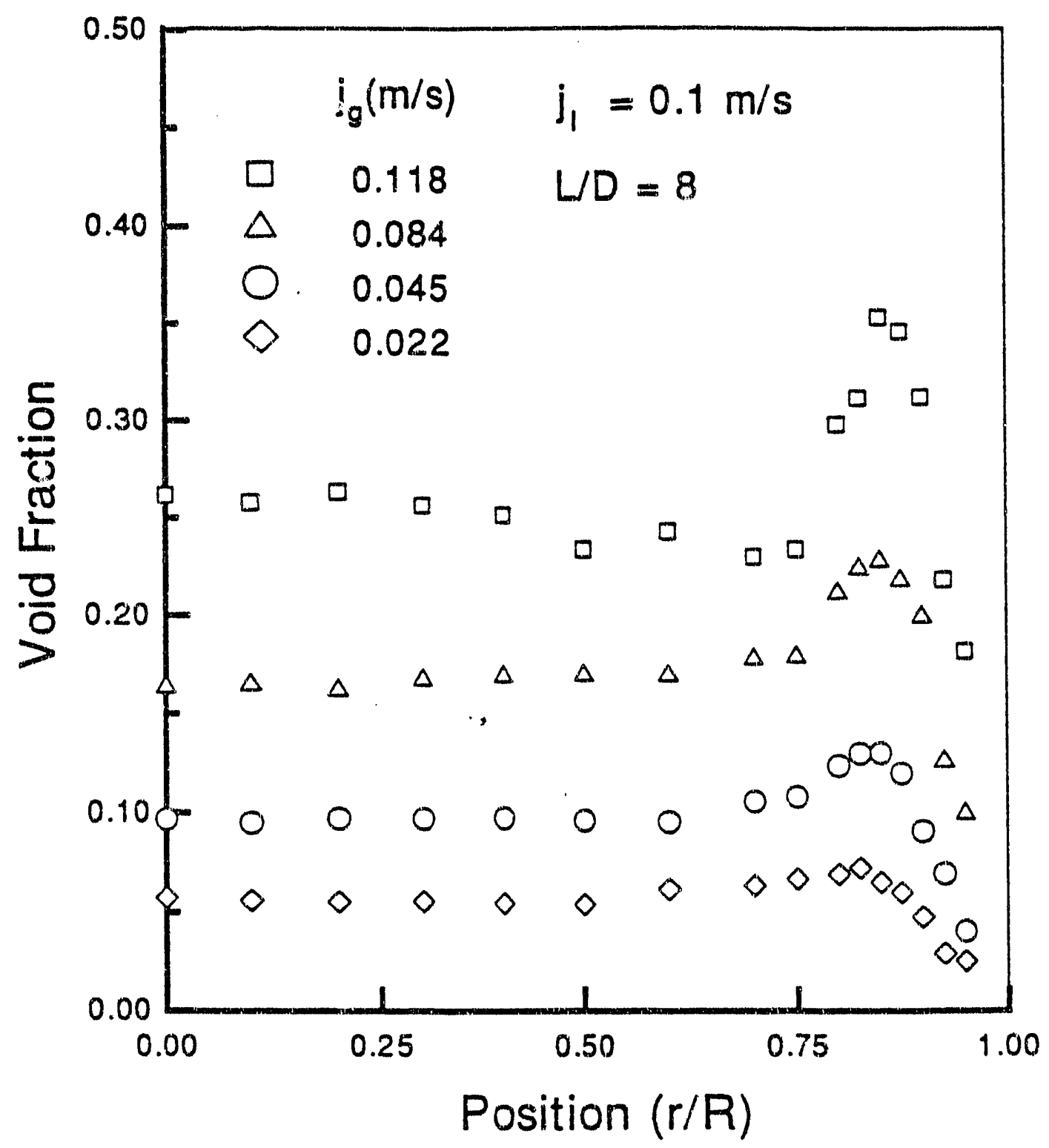

Fig. 19.a. The radial profile of void fraction for air-water bubbly flow at $j_{1}=0.1 \mathrm{~m} / \mathrm{s}$ and $L / D=8$. 

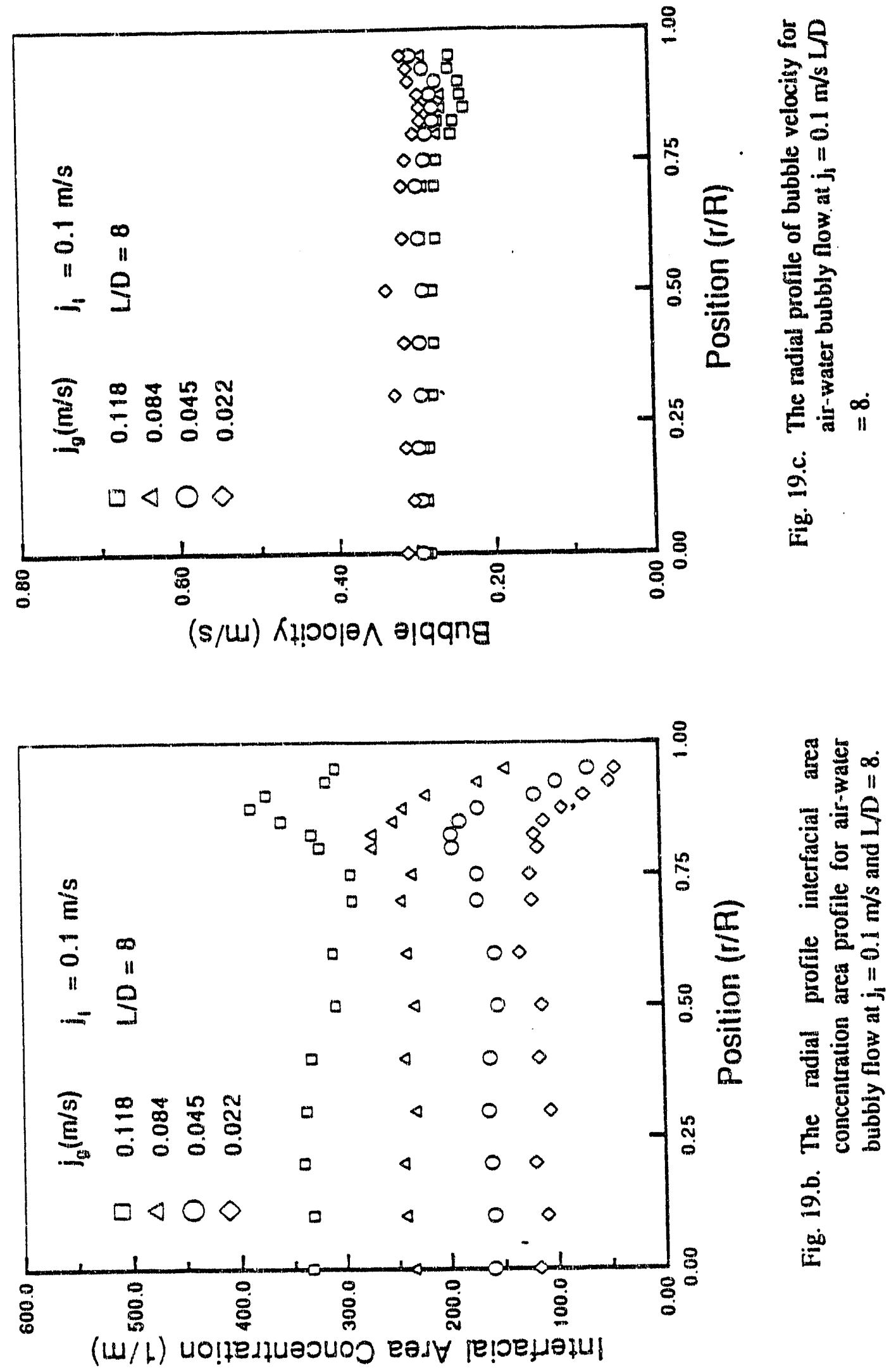

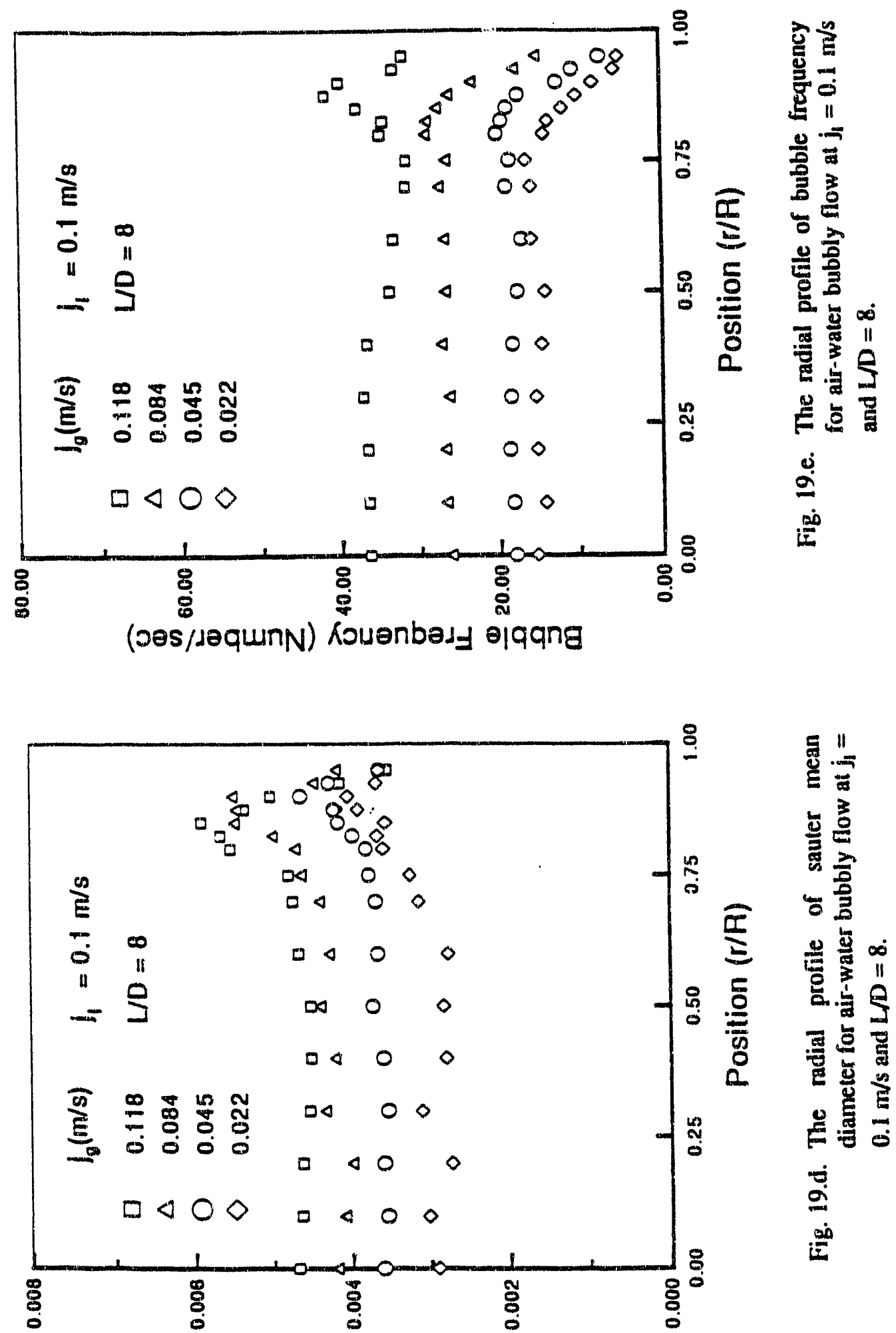

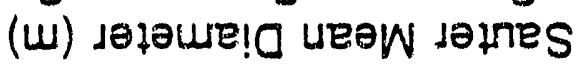




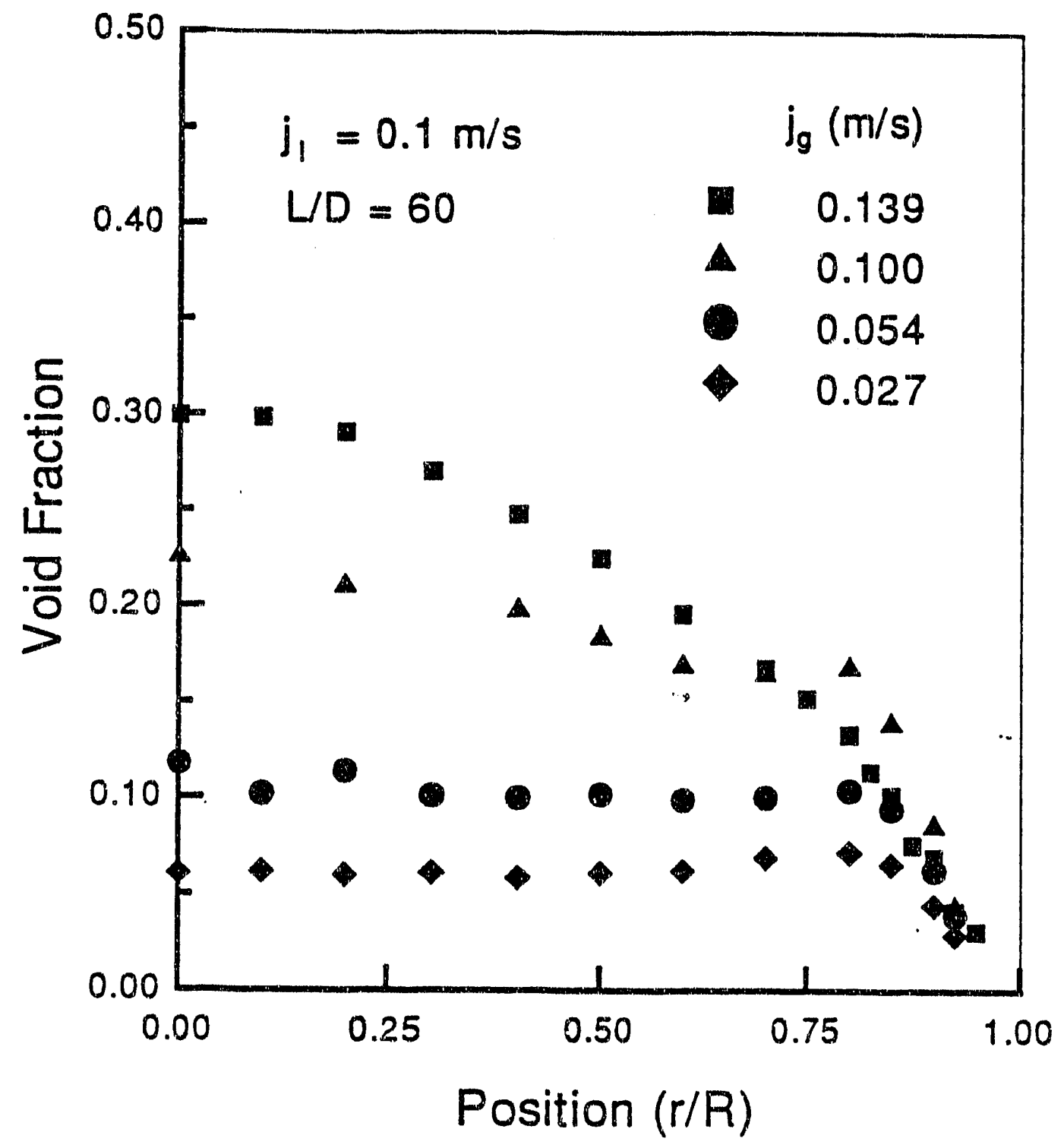

Fig. 20.2. The radial profile of void fraction for air-water bubbly flow at $j_{1}=0.1 \mathrm{~m} / \mathrm{s}$ and $L / D=60$. 

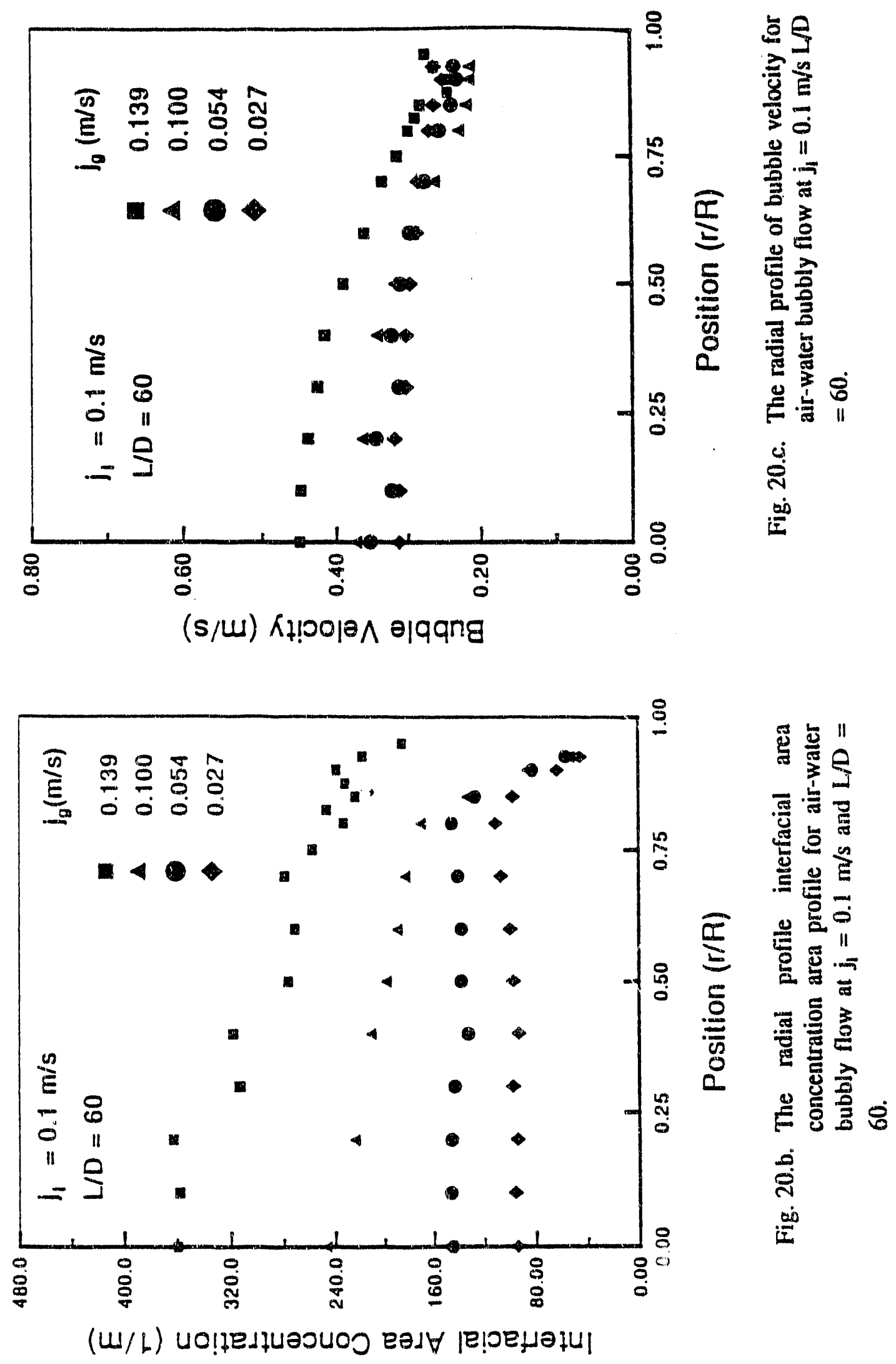

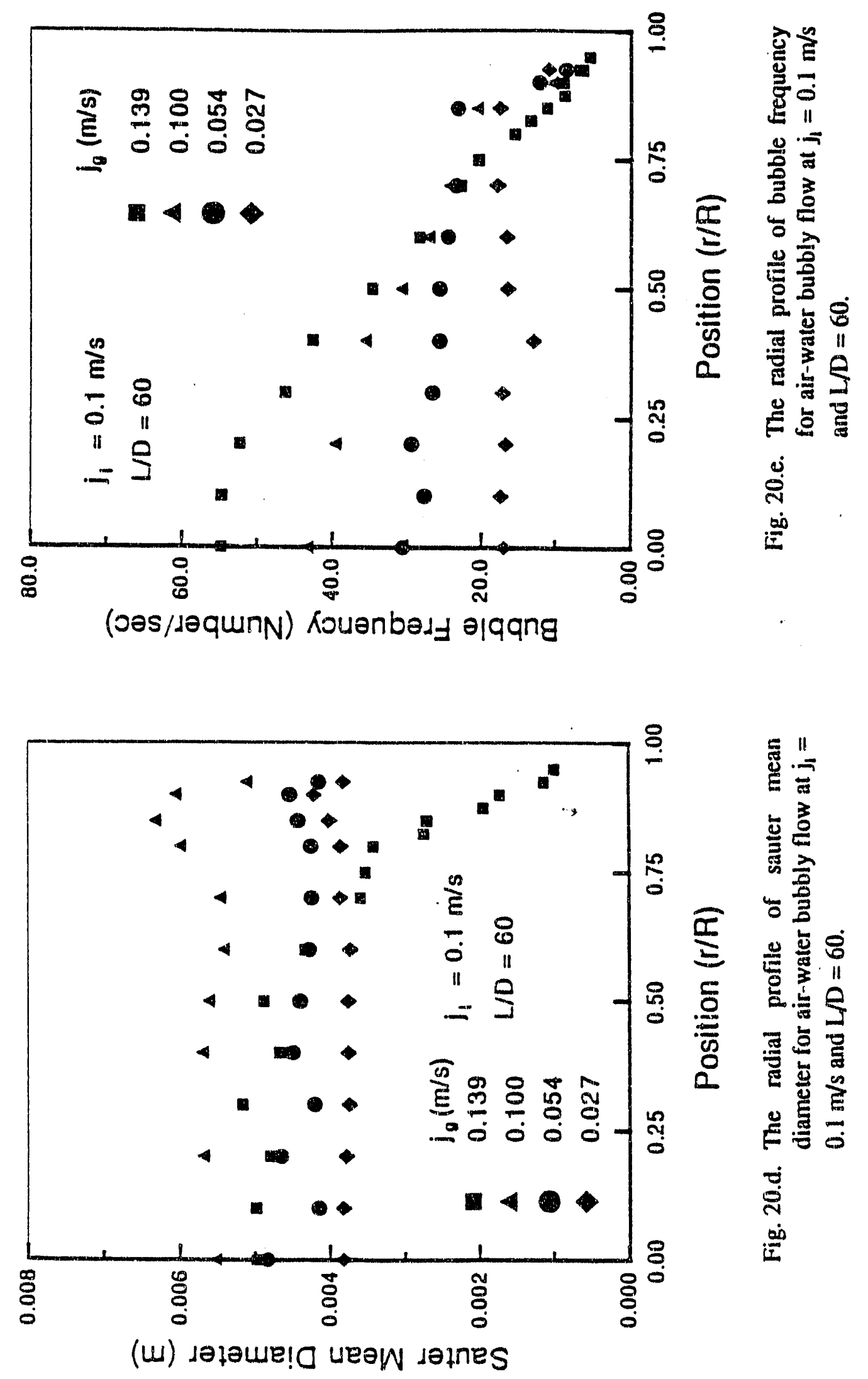
of the discontinuity. The jump gets smaller and smaller as the superficial liquid velocity is reduced. The phasic character at $L / D=60$ is almost the same as the entrance region except it shows a flatter profile at the center core.

The $j_{l}=0.10 \mathrm{~m} / \mathrm{s}$ case behaves quite differently from the othiers. At $L / D=8$, the radial void distribution also shows a typical bubbly flow profile, near wall peaking. In the $j_{g}=$ $0.118 \mathrm{~m} / \mathrm{s}$ case, the peak of void fraction is exceeding the limit of $30 \%$ [9] for the slug flow transition. The near wall peak diminishes as $j_{g}$ decreases at the entrance region. When the two-phase fluid reaches the axial distance $L / D=60$, the near wall peak reduces to a negligible value compared to the center core void. The void fraction distributes quite evenly at the center core and decreases as it approaching the pipe wall for the $j_{g}=0.054$ and $0.027 \mathrm{~m} / \mathrm{s}$ cases. On the other hand, for the $j_{g}=0.118$ and $0.084 \mathrm{~m} / \mathrm{s}$ cases, the void peaks at the center core which is the character observed at the slug flow and at the transient. As a matter of fact, the bubbles agglomerate together to form a bubbly slug at the developed region but there is not a single slug bubble observed in these experiments. The bubble frequency plots confirm that the void peaks at core region due to higher bubble concentration at the center-line. If the flow goes further downstream, it probably will change to slug flow. However, the test section is too short for the slug flow to be developed. The Interfacial area concentration goes much the same way as the void fraction both at $L / D=8$ and $L / D=60$. The sauter mean diameter measurements are quite scattered but in average they are larger than the case of higher liquid velocity cases and range from roughly $3 \mathrm{~mm}$ to $6 \mathrm{~mm}$. At $\mathrm{L} / \mathrm{D}$ $=60$, the average sauter mean does grow bigger in all regions. This may indicate that the bubbles coalesce as the flow is developing.

\subsubsection{Constant Gas Velocity}

In the second sequence of plots (see Fig.21 to 24), data is grouped by their superficial gas velocity. In each group of the plots, the gas injected into the system is the same but the carrying liquid has different flow rates. The higher the liquid velocity, the lower the average void will be, which is shown in the void fraction plots. At the entrance, the relation is correct in both the core region and the near wall region. In the developed region, the relation is generally true. However, if we examine the plot a litule more carefully, we find that in the $j_{l}=1.0 \mathrm{~m} / \mathrm{s}$ cases the void peaks higher than in the $j_{l}=0.6 \mathrm{~m} / \mathrm{s}$ cases in both gas velocities 


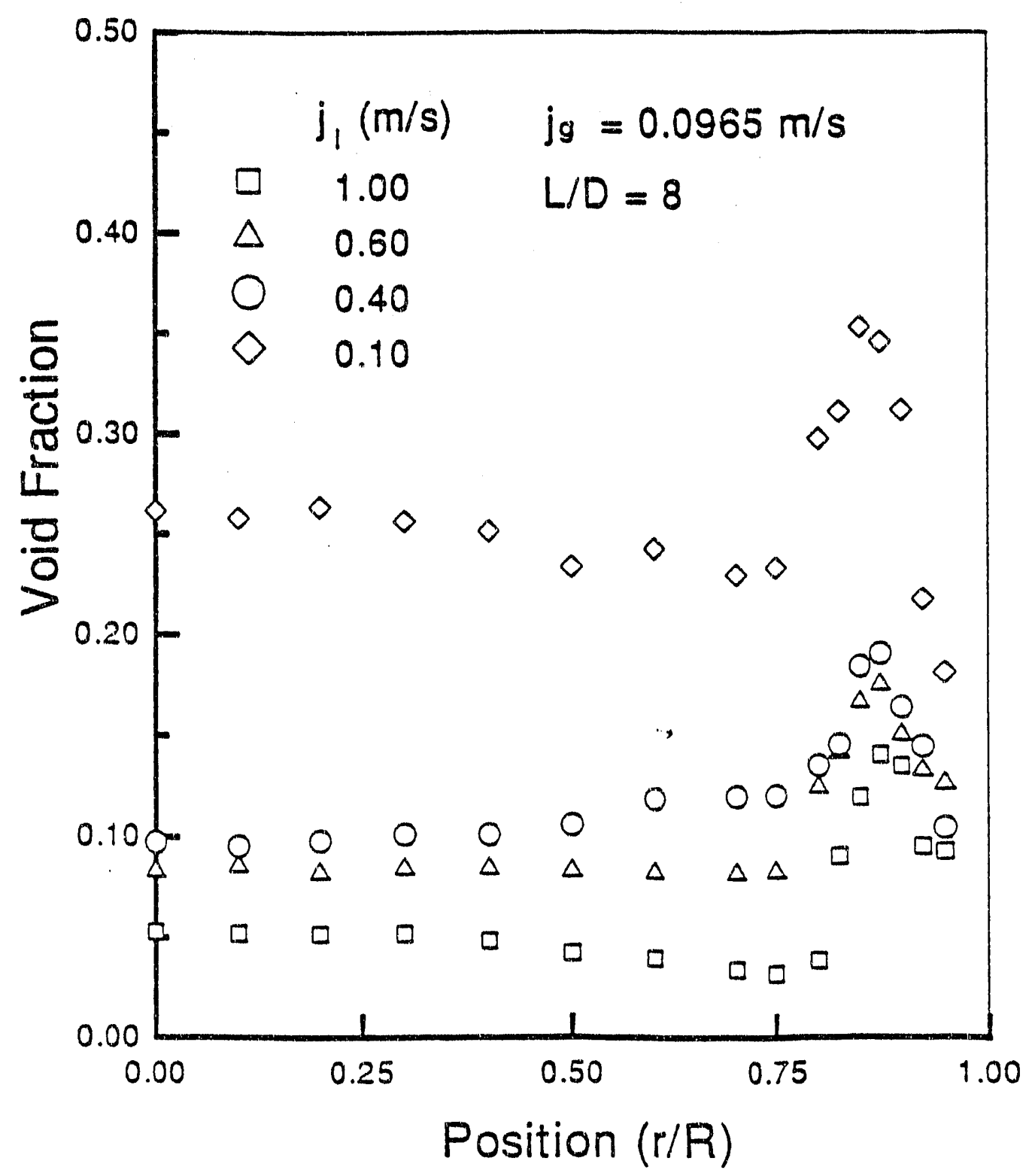

Fig. 21.a. The radial profile of void fraction for air-water bubbly flow at $\mathrm{j}_{\mathrm{g}}=0.0965 \mathrm{~m} / \mathrm{s}$ and $I / D=8$. 

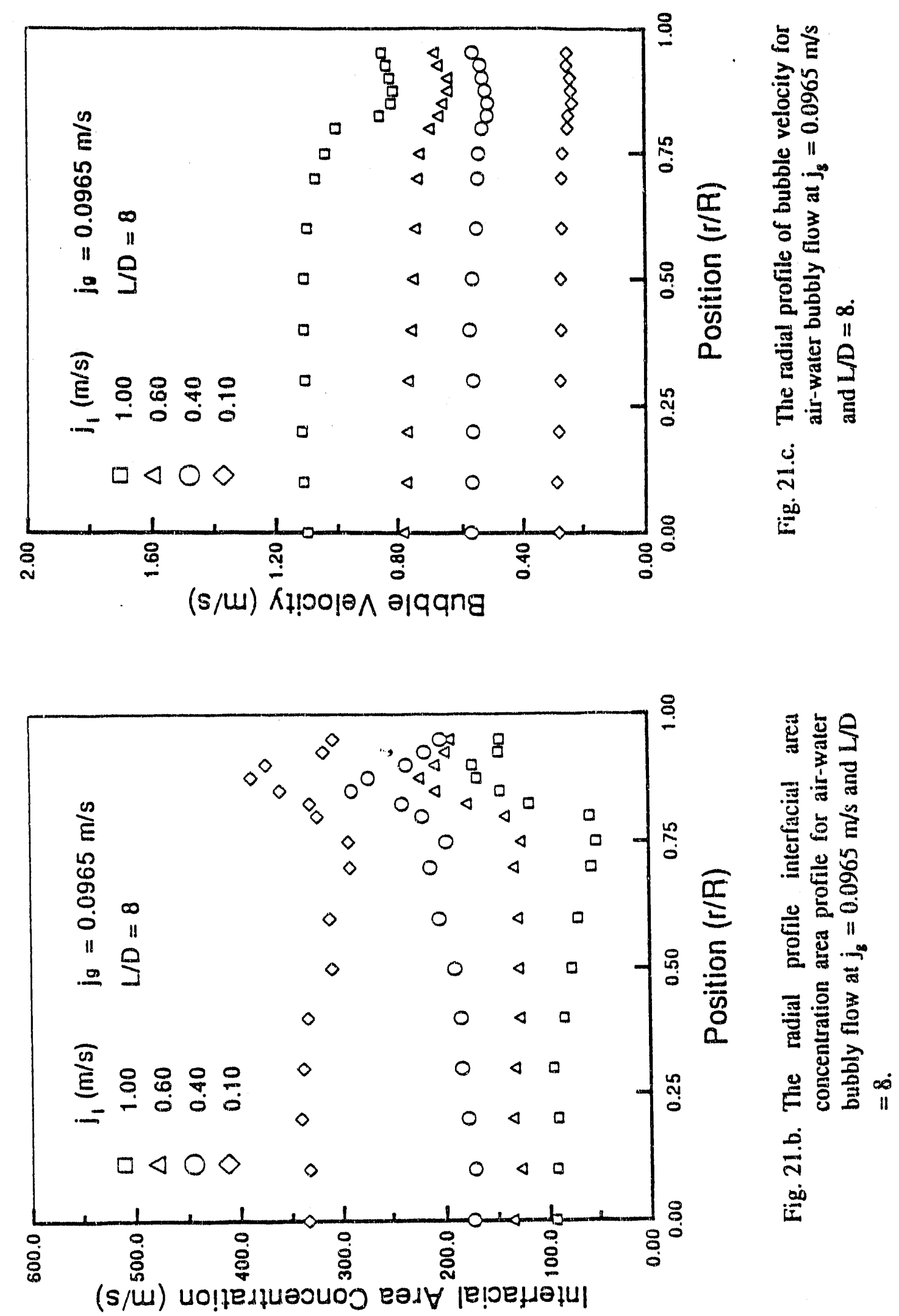

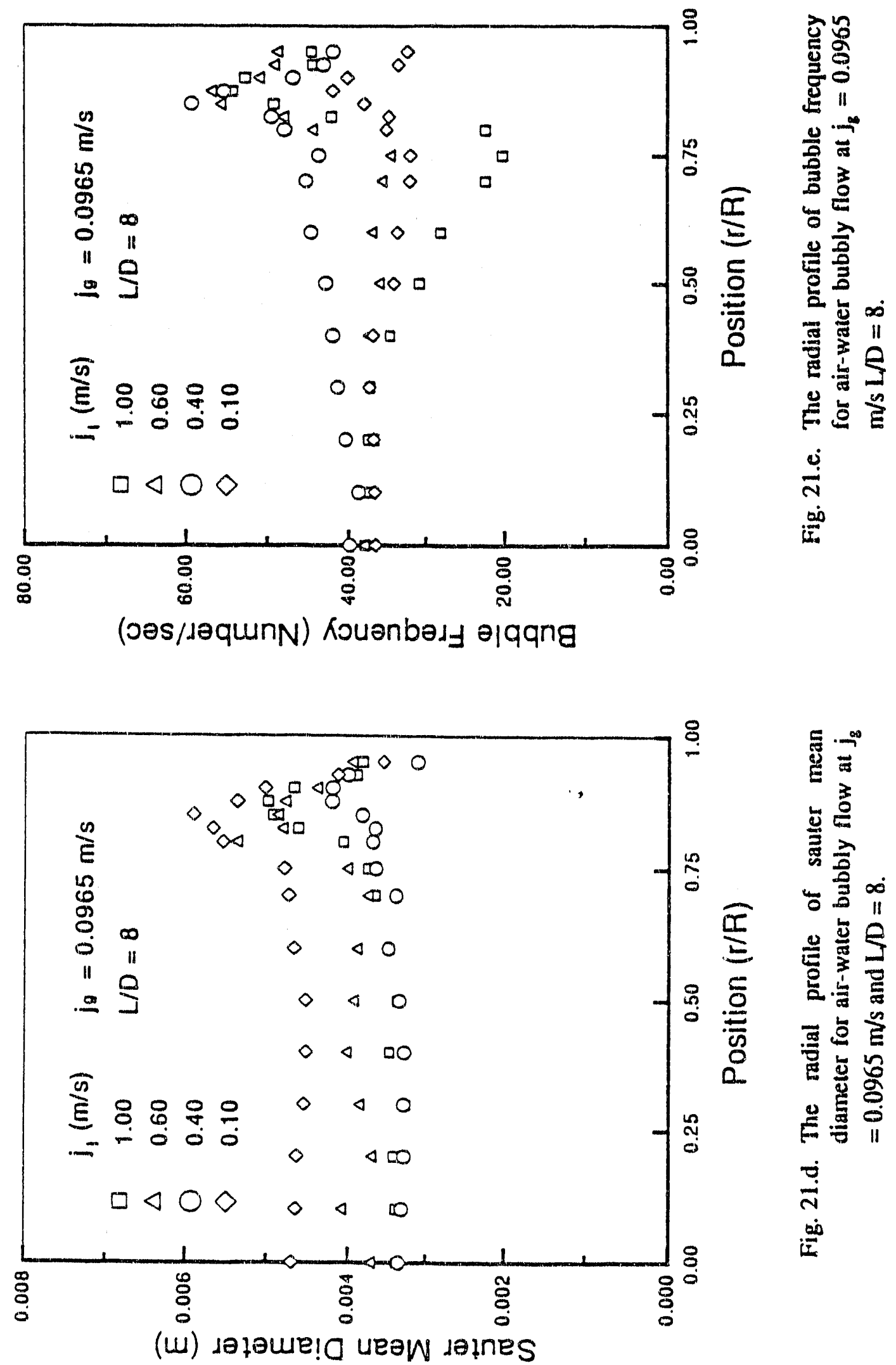


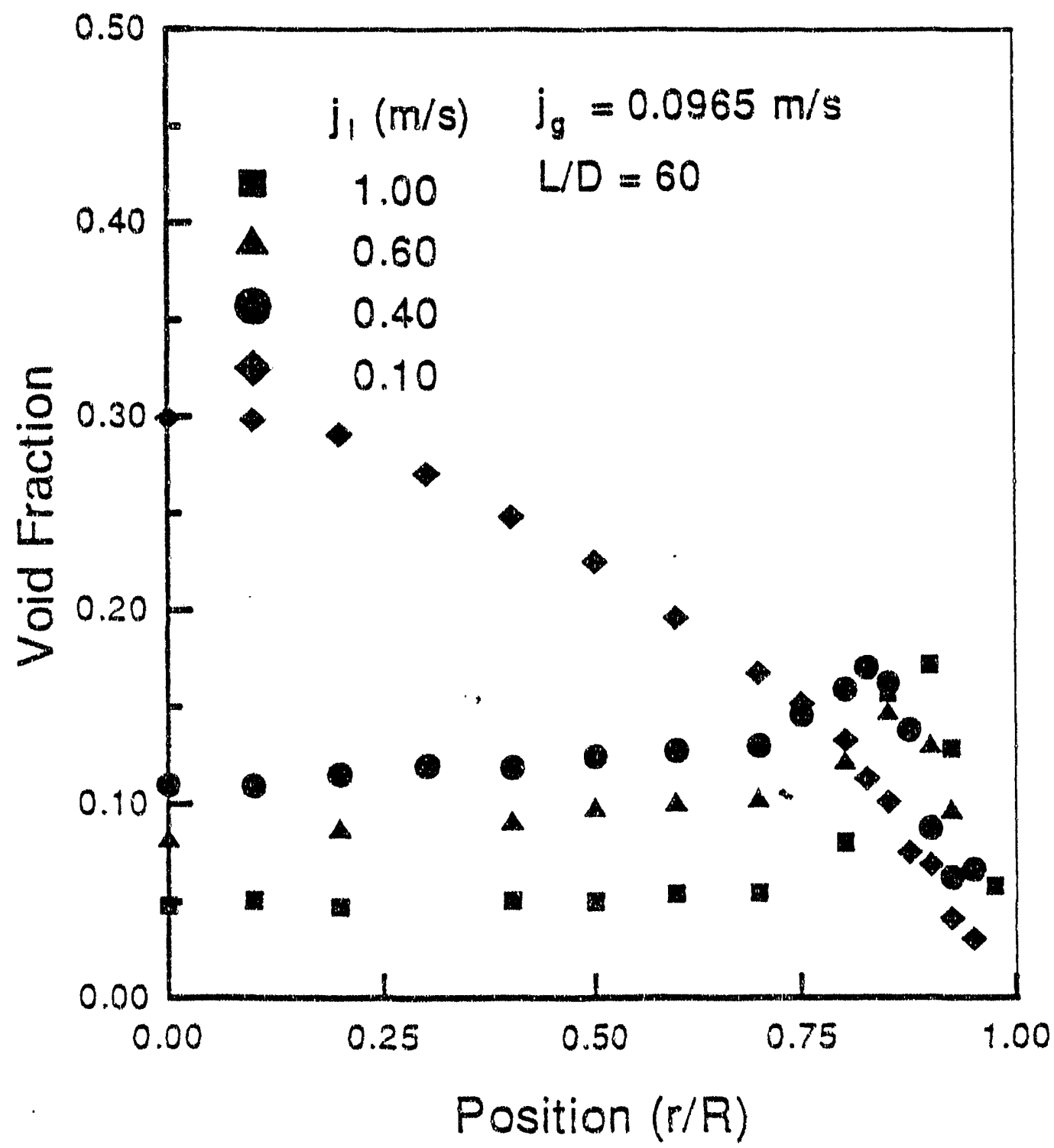

Fig. 22.a. The radial profile of void fraction for air-water bubbly flow at $j_{8}=0.0965 \mathrm{~m} / \mathrm{s}$ and $L D=60$. 

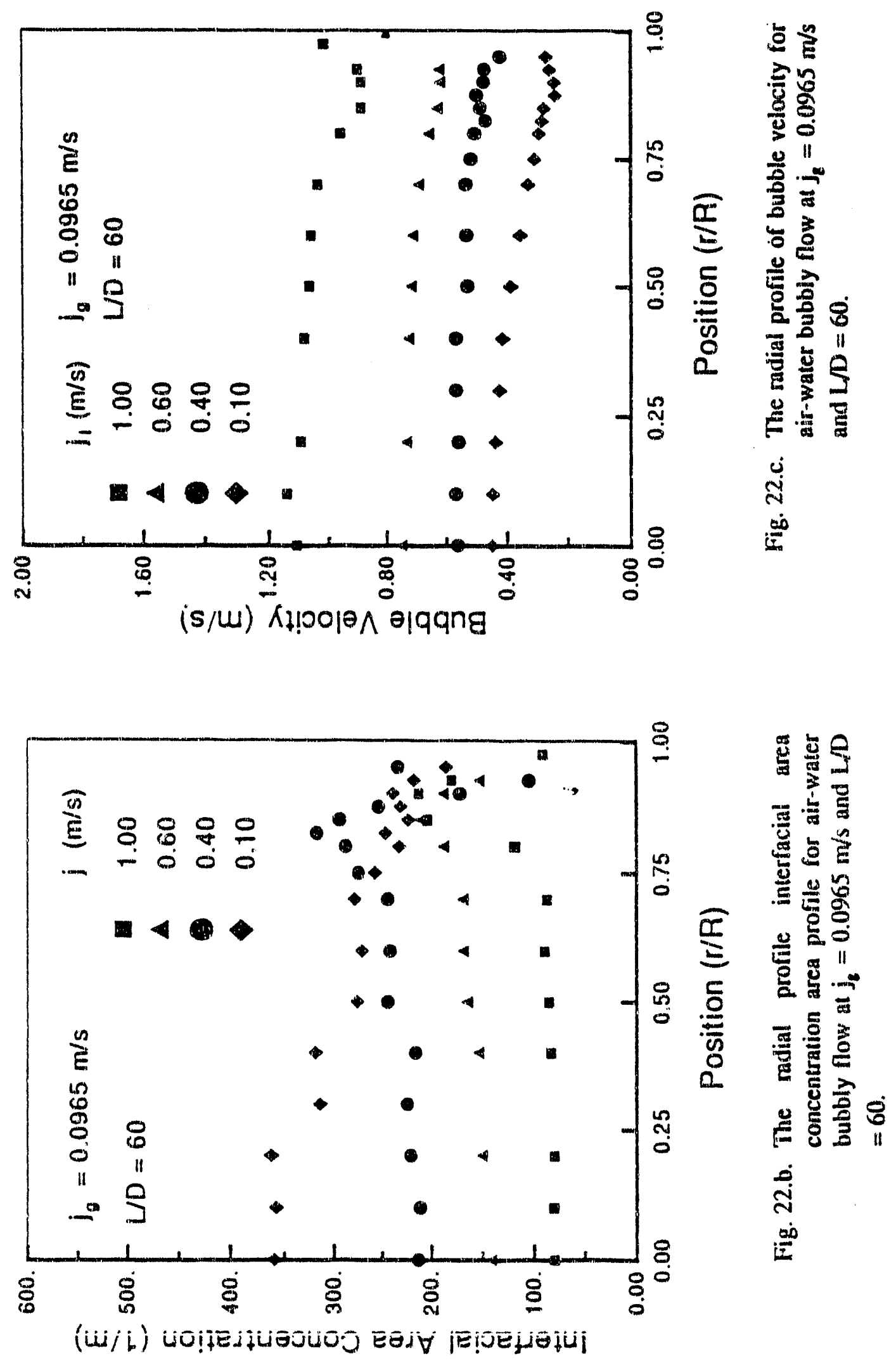

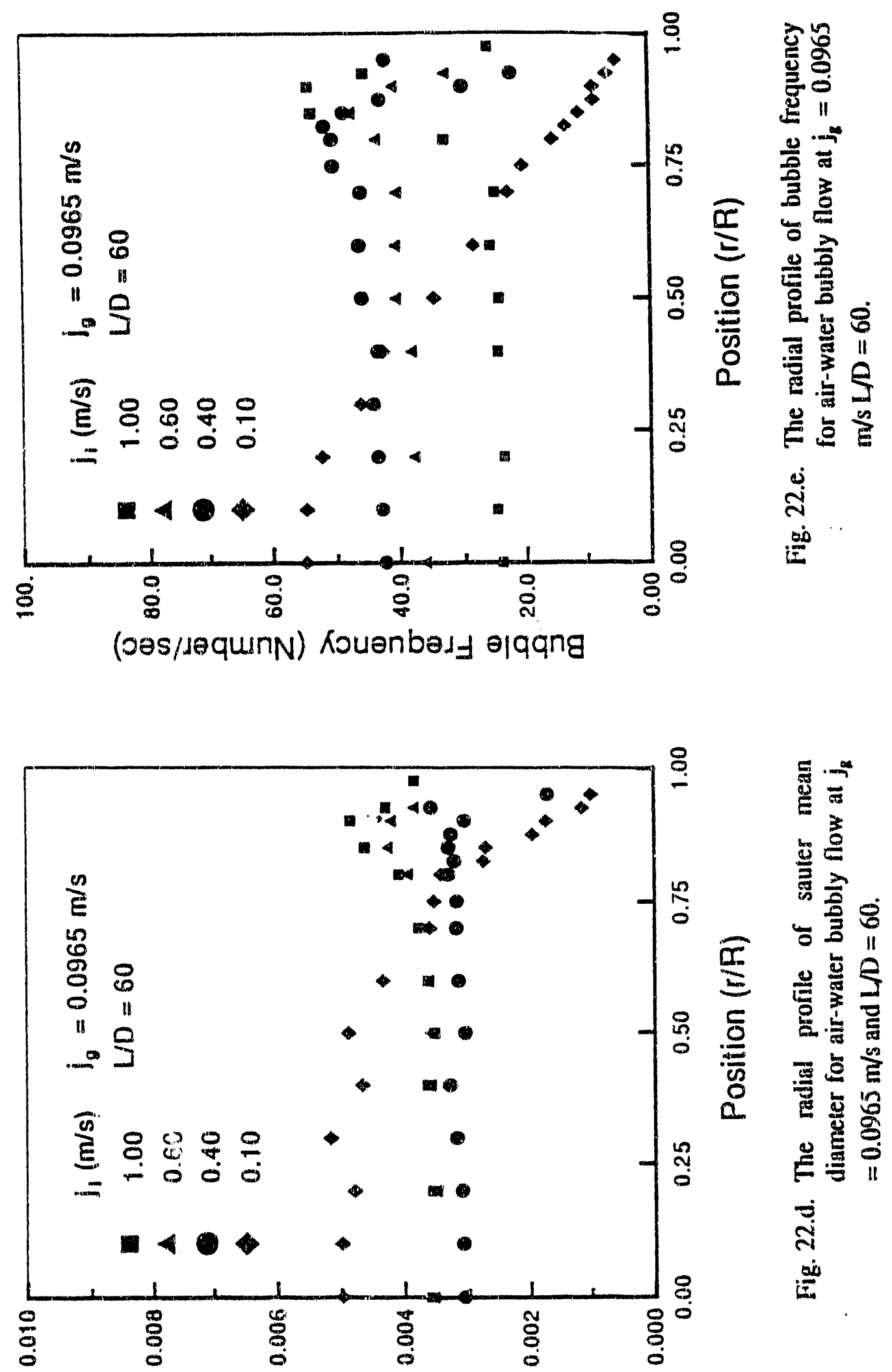

(uj) dafaue! ue⿰W jafries 


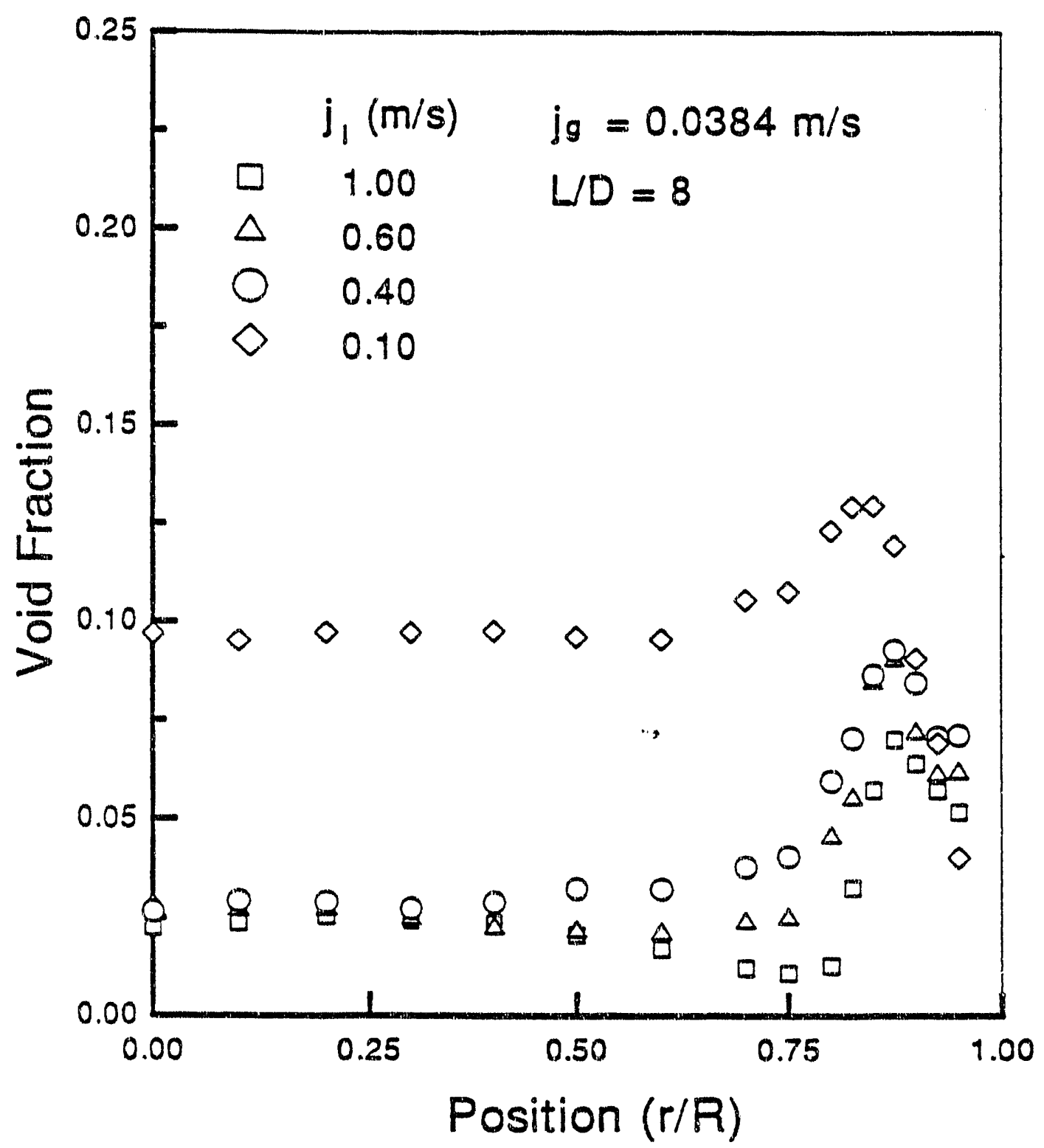

Fig. 23.a. The radial profile of void fraction for air-water bubbly flow at $j_{3}=0.0384 \mathrm{~m} / \mathrm{s}$ and $L / D=8$. 

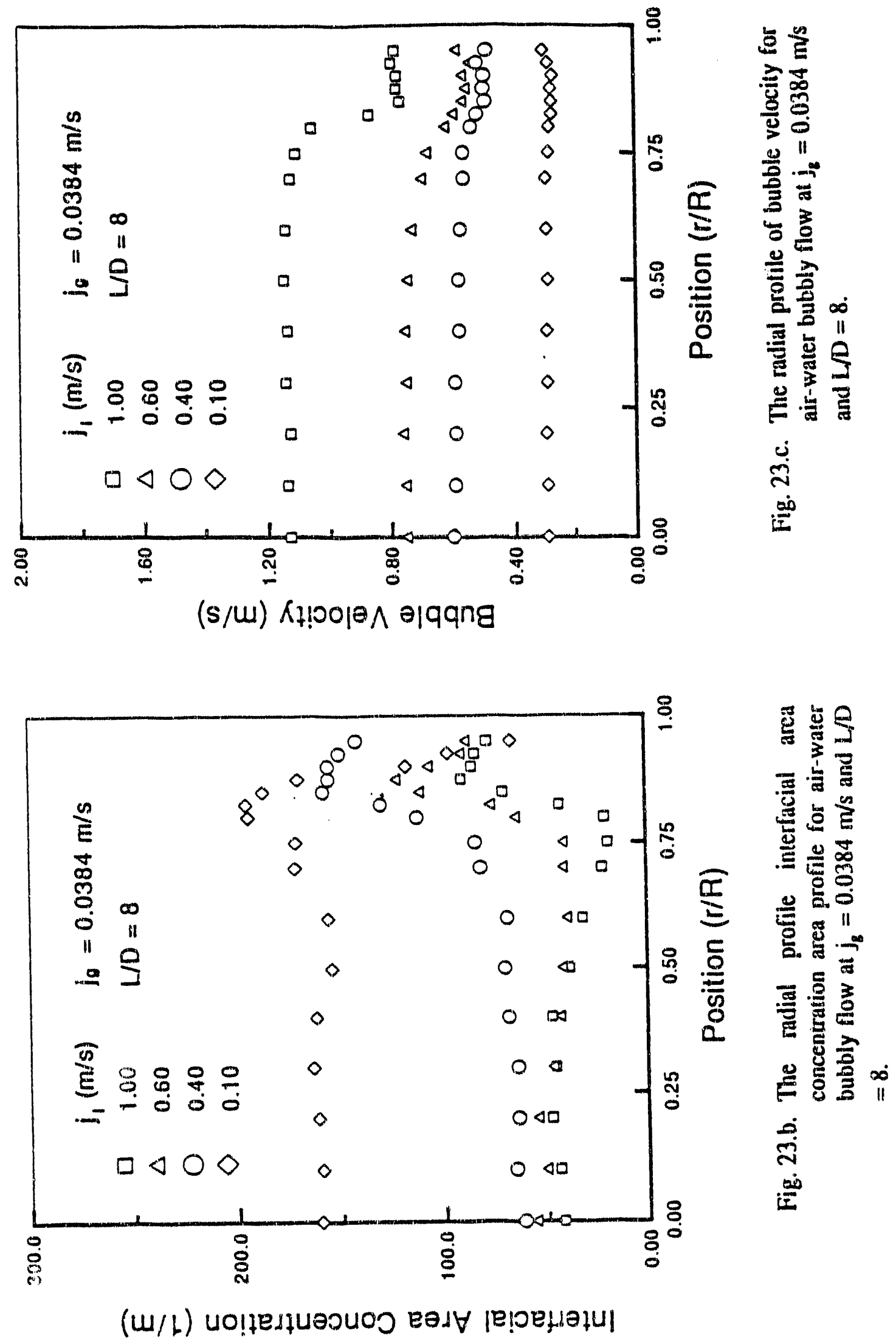

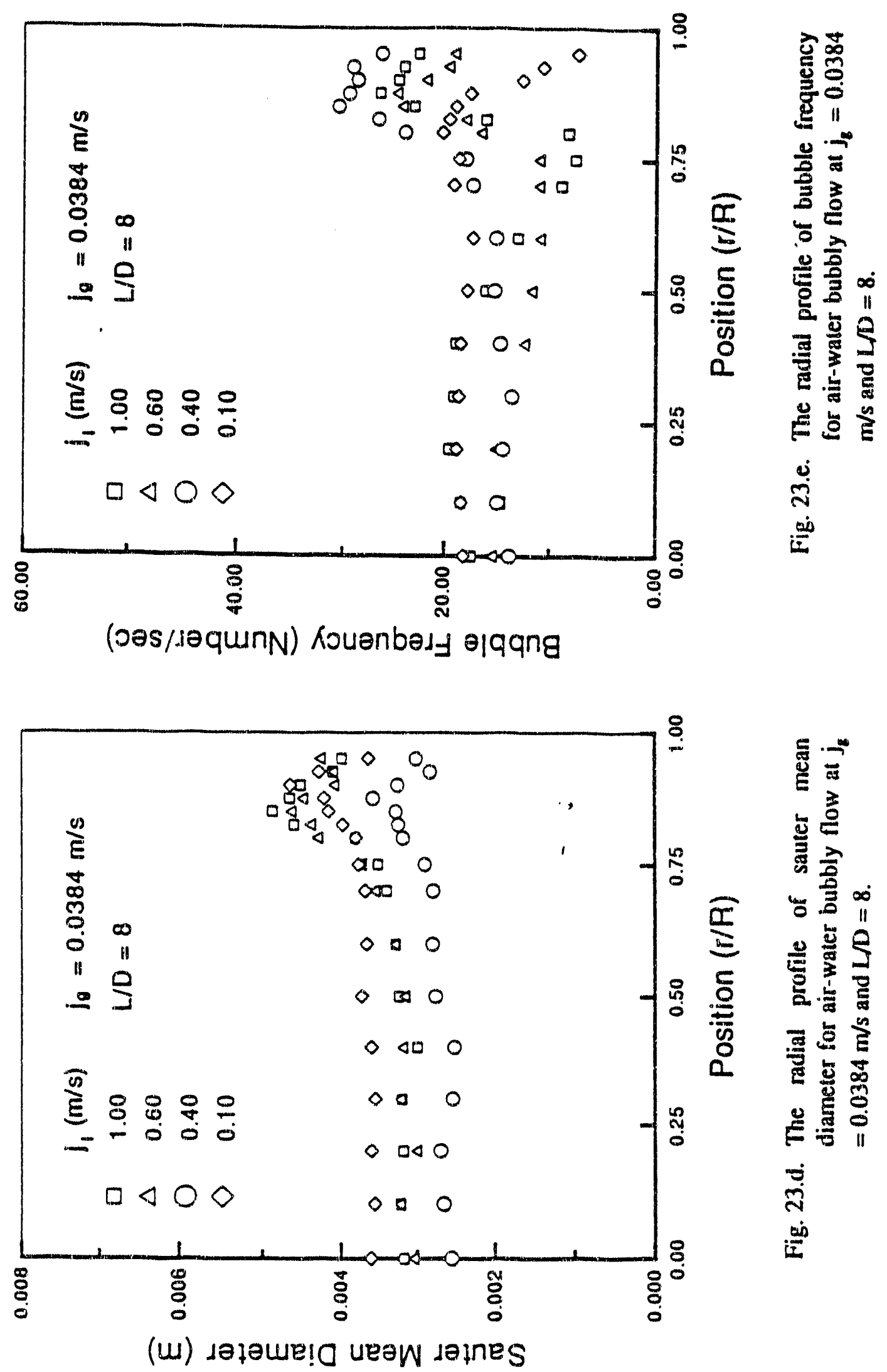


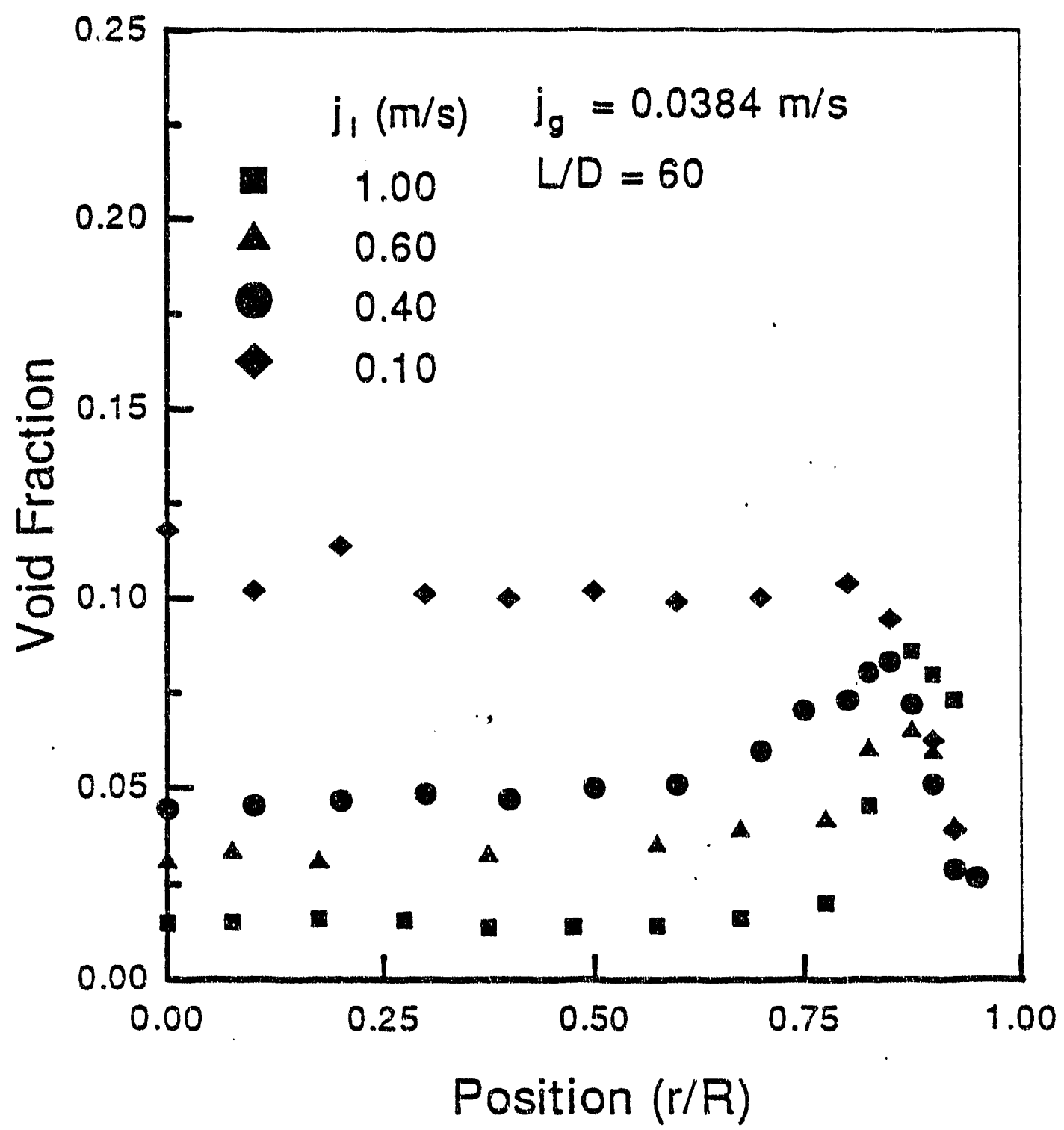

Fig. 24.2. The radial profile of void fraction for air-water bubbly flow at $j_{8}=0.0384 \mathrm{~m} / \mathrm{s}$ and $L / D=60$. 

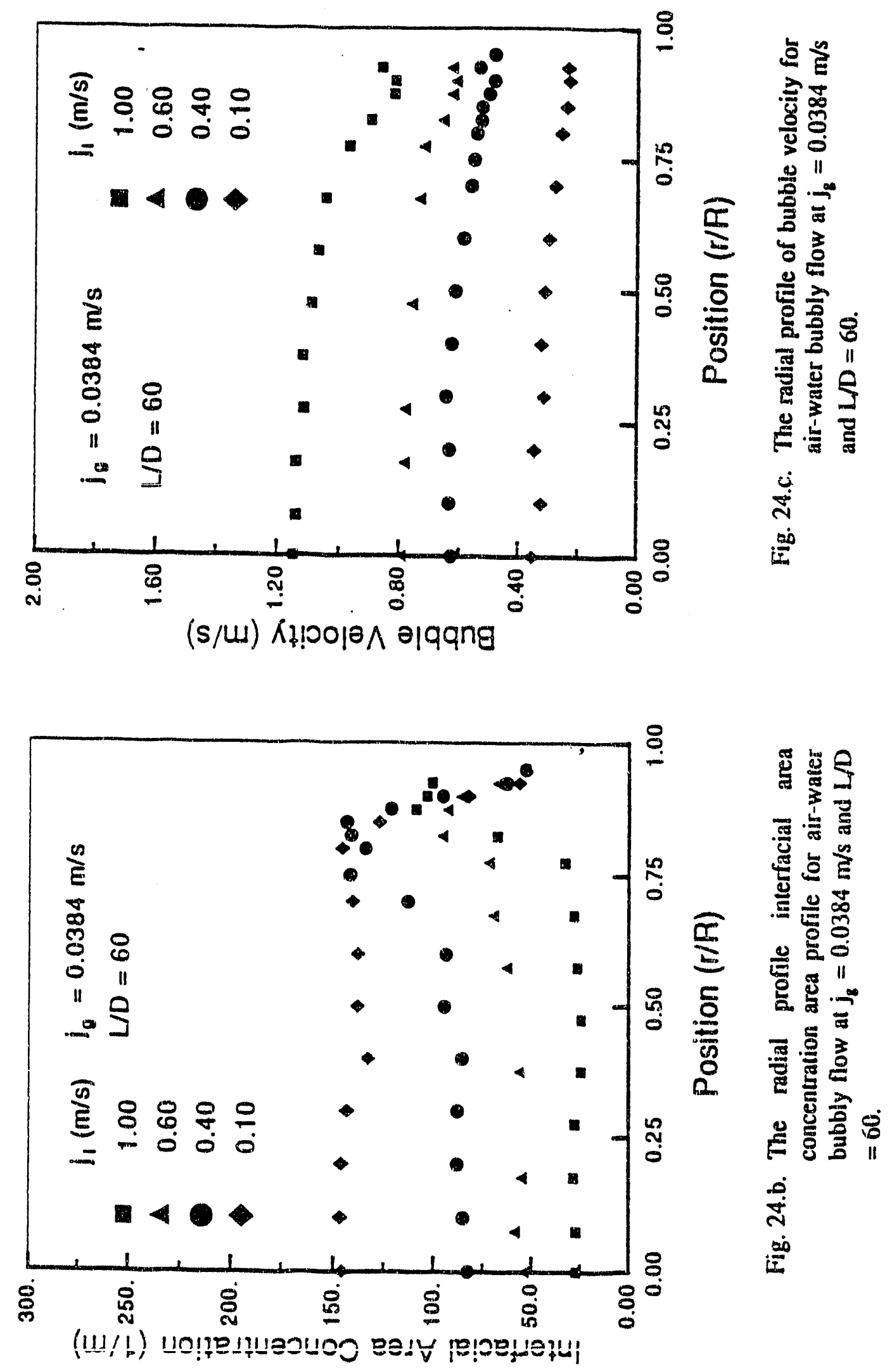

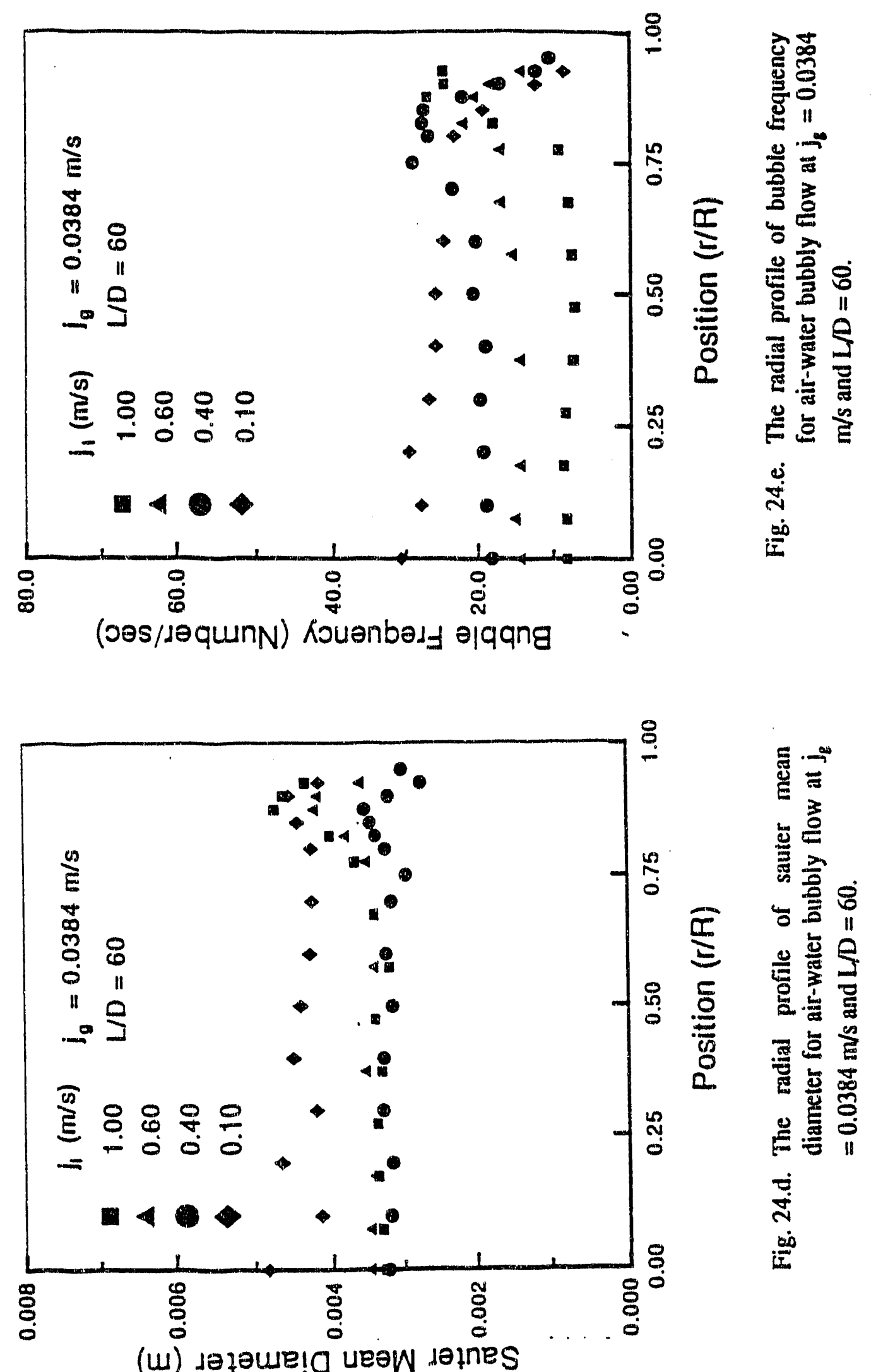
while their average void fractions are lower than in the $0.6 \mathrm{~m} / \mathrm{s}$ cases. This shows that the liquid velocity can push the higher void peaking at the fully developed region. A more detailed analysis will be given in the average analysis section.

The sauter mean diameter plots show no observable trend of dependence on the liquid flow rate. However, in the $j_{l}=0.1 \mathrm{~m} / \mathrm{s}$ cases, the measured sauter mean diameters are consistently larger than the others, both at the entrance and fully developed region. One more remark, in the $j_{l}=0.1 \mathrm{~m} / \mathrm{s}$ and the $j_{g}=0.118 \mathrm{~m} / \mathrm{s}$ case, the sauter mean diameter decreases monotonically as it approaches the pipe wall which is different from the rest of the cases.

The radial velocity profile more clearly shows that there is a velocity discontinuity at roughly $r / R=0.775$ in all cases. It becomes steeper and more obvious in higher liquid velocity flow. Furthermore, one can see that the measured bubble velocity goes up a little as the probe moves closer to the wall in all cases and in both the entrance and fully developed region. Once we thought that the increment was due to measurement error, however, this may not be the case. Because the near wall velocity distribution does not show much difference from the center core distribution and it is very repeatable measurement. Furthermore, the interfacial area concentration measurement relies on the velocity measurement which shows a consistent behavior at the near wall region. Therefore the measurement error argument doesn't stand. It is suspected that the bubble actually moves faster when it get closer to the wall. Because a thin liquid film acts like a lubricant so that bubbles can slide faster along the pipe wall. More evidence is needed to prove this case.

\subsubsection{The Comparison Plots}

The comparison plots are grouped by the liquid velocity. The detailed radial profile comparisons are self-explanatory. However, a general discussion is given in a few cases. First the radial void profile, void peak is getting smaller in the fully developed region than at the entrance region in most of the cases. However, in the $j_{l}=1.0 \mathrm{~m} / \mathrm{s}$ case, the void peak is getring bigger in the fully developed region. It seems in higher liquid velocity cases the bubble is pushed to the wall region as the flow is developing. 

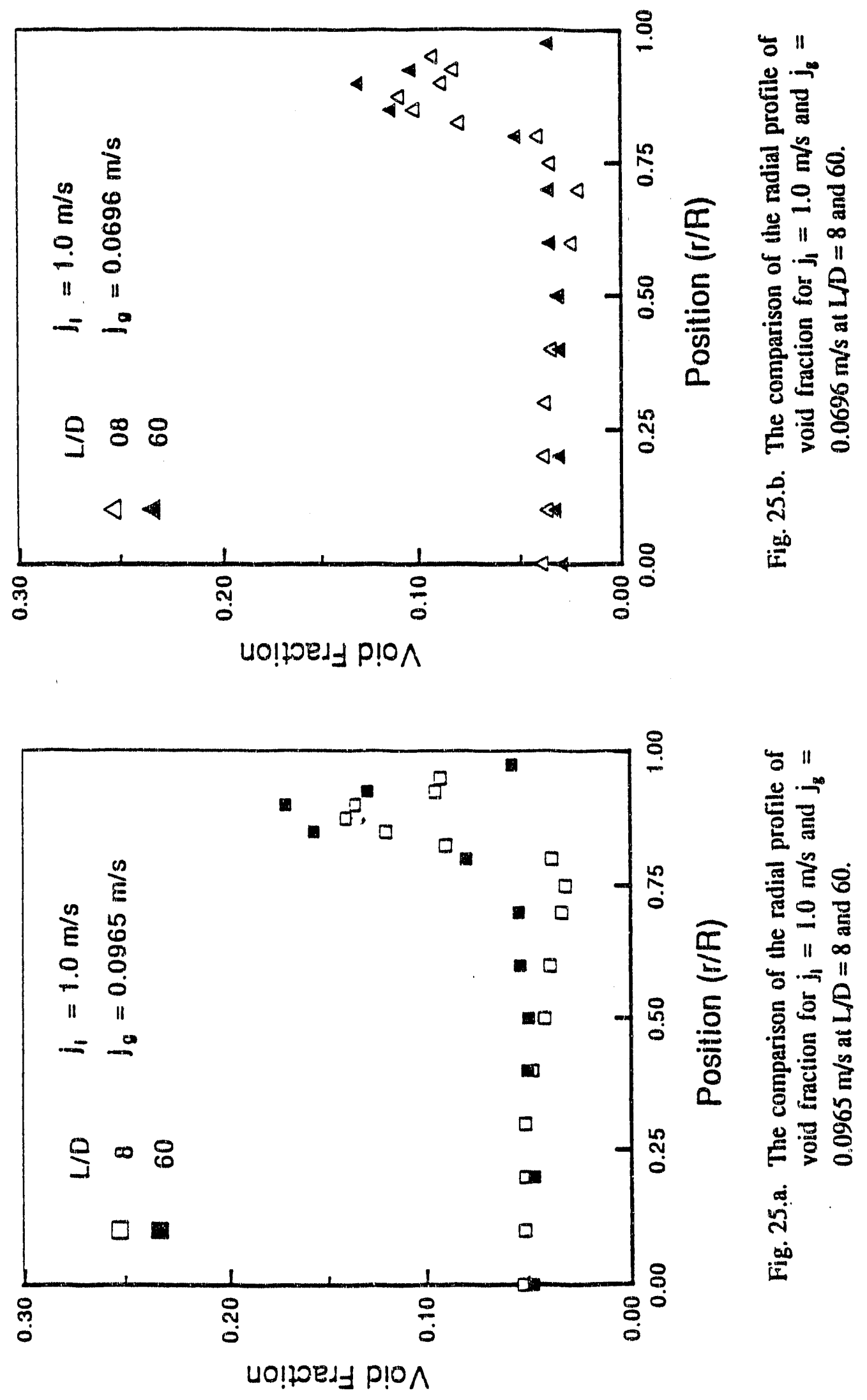

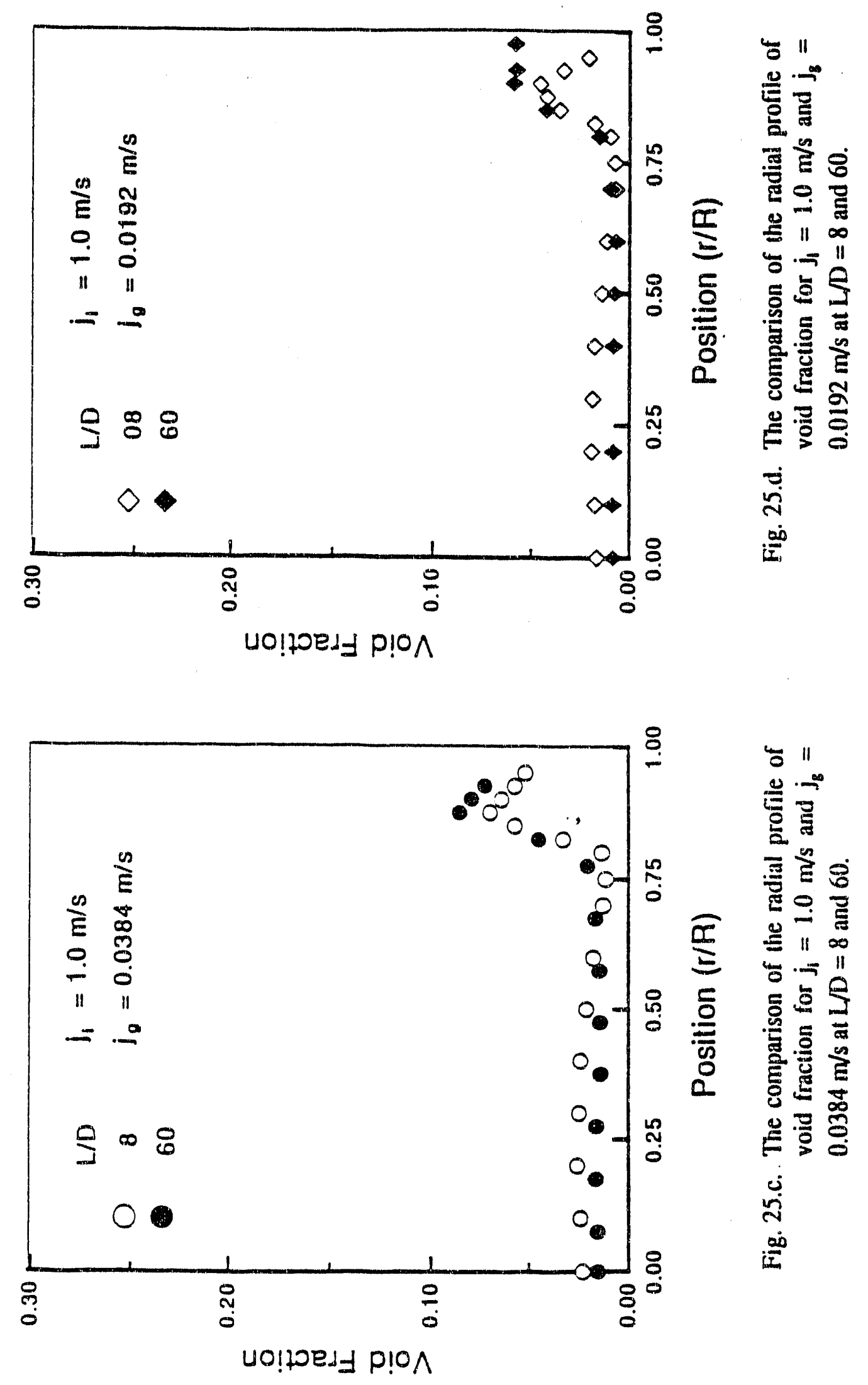

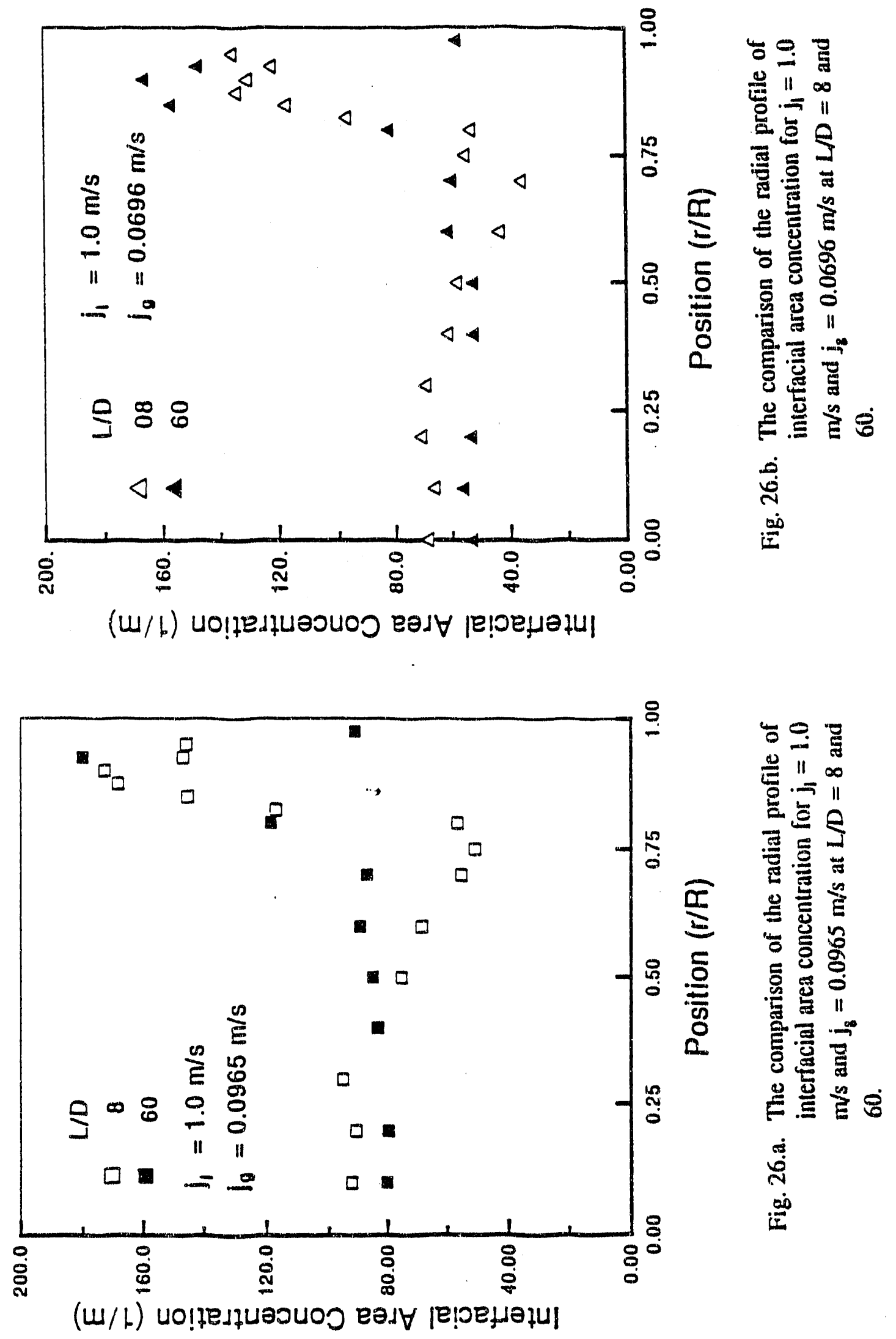

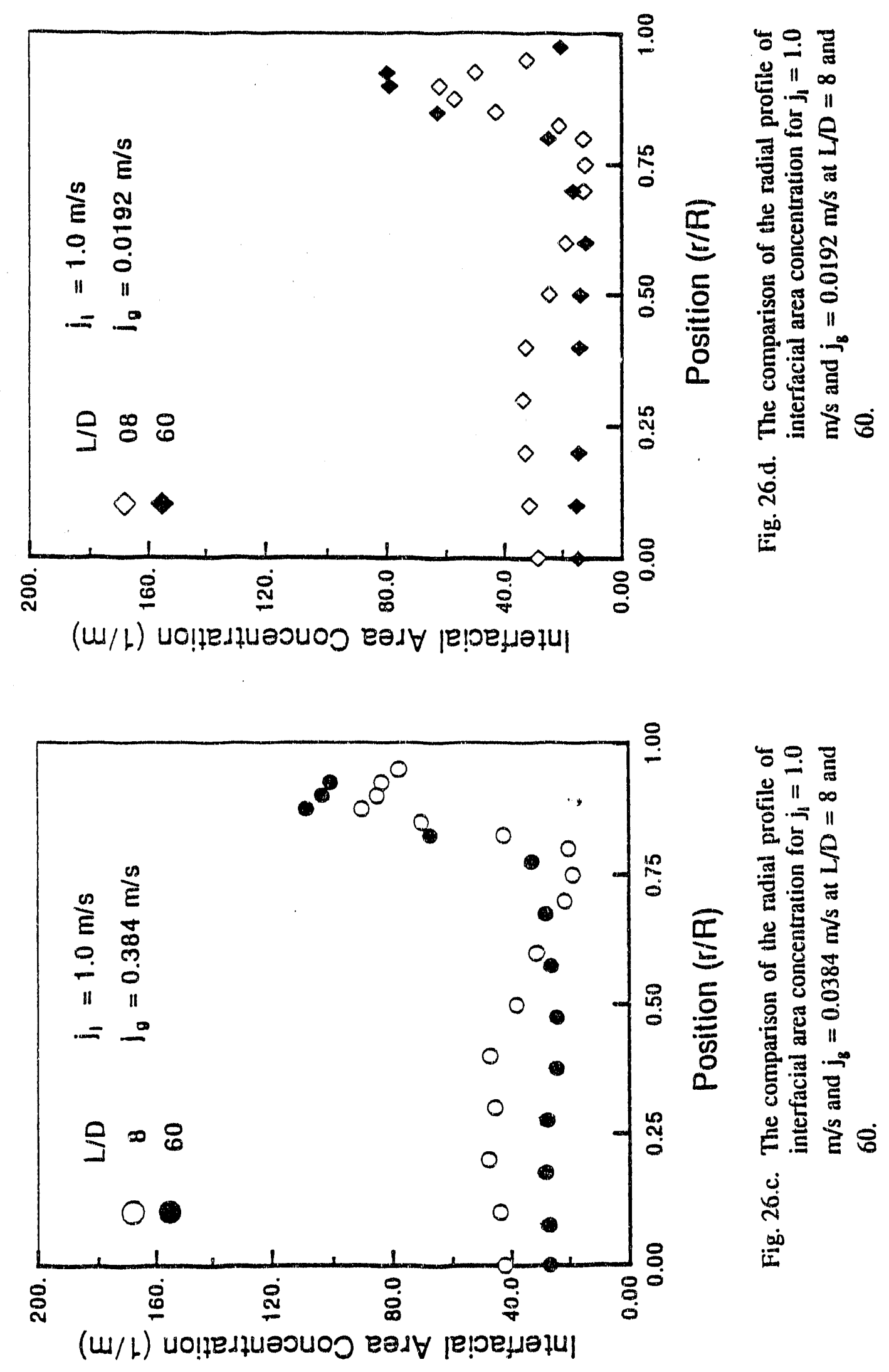

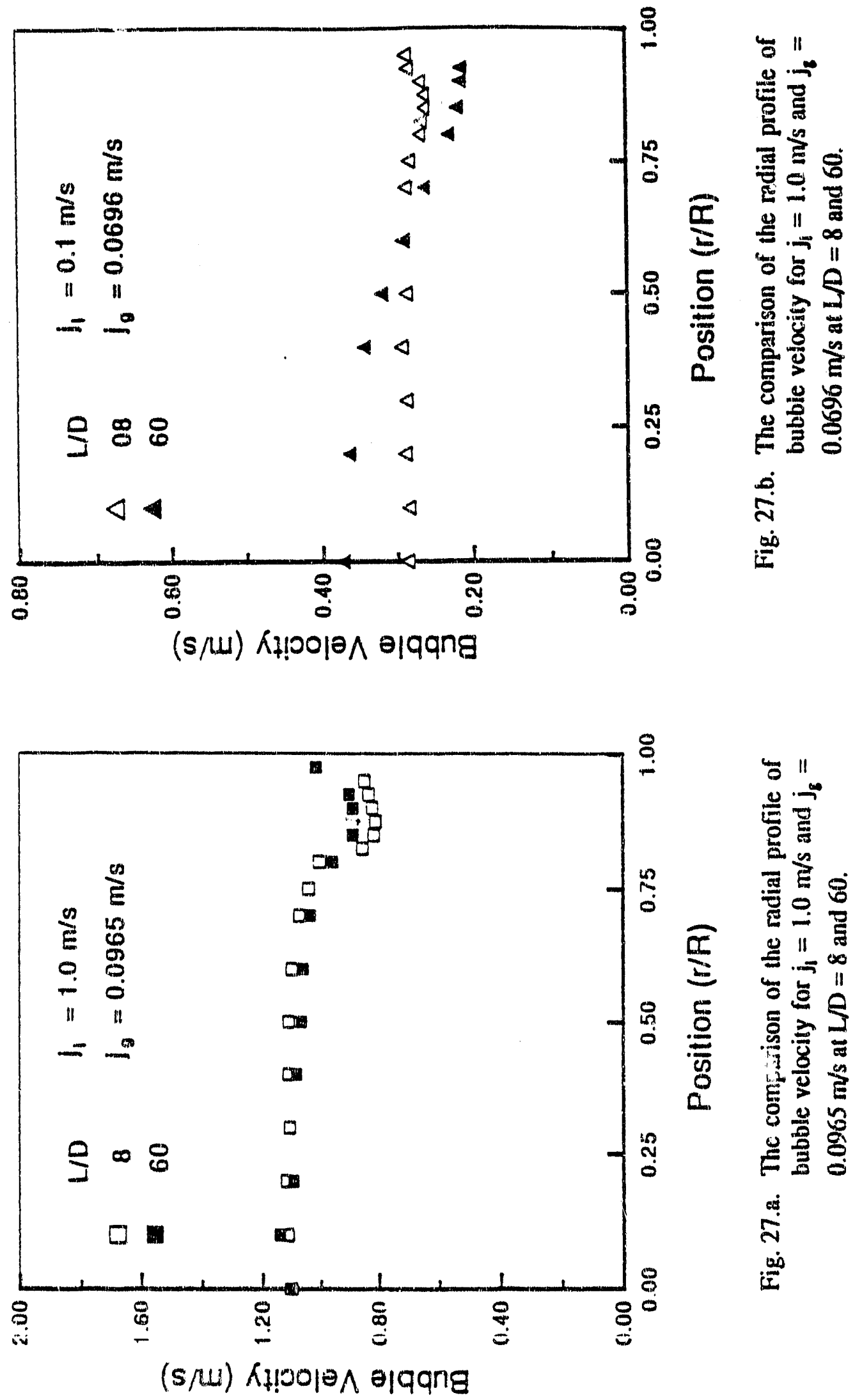

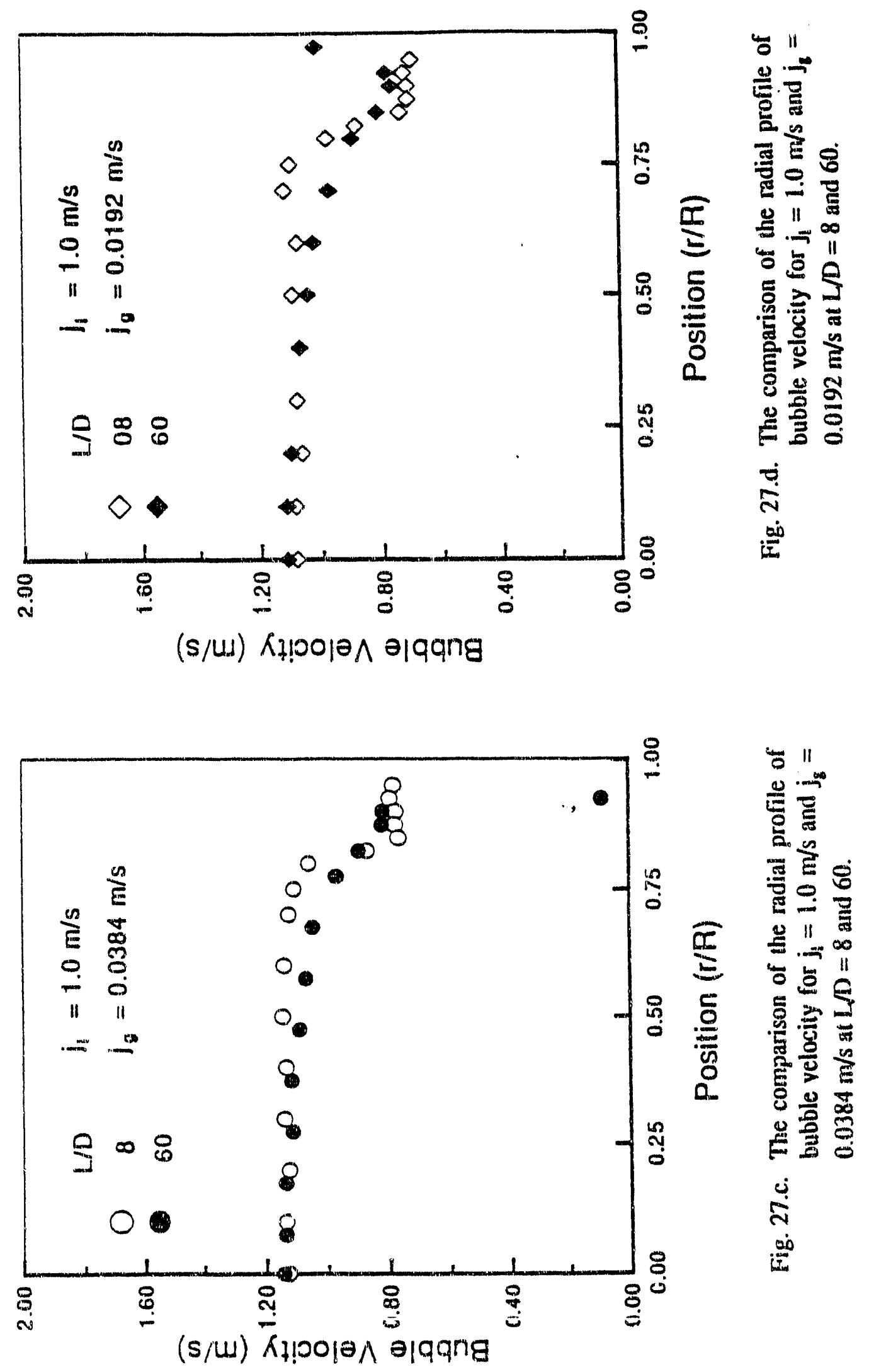


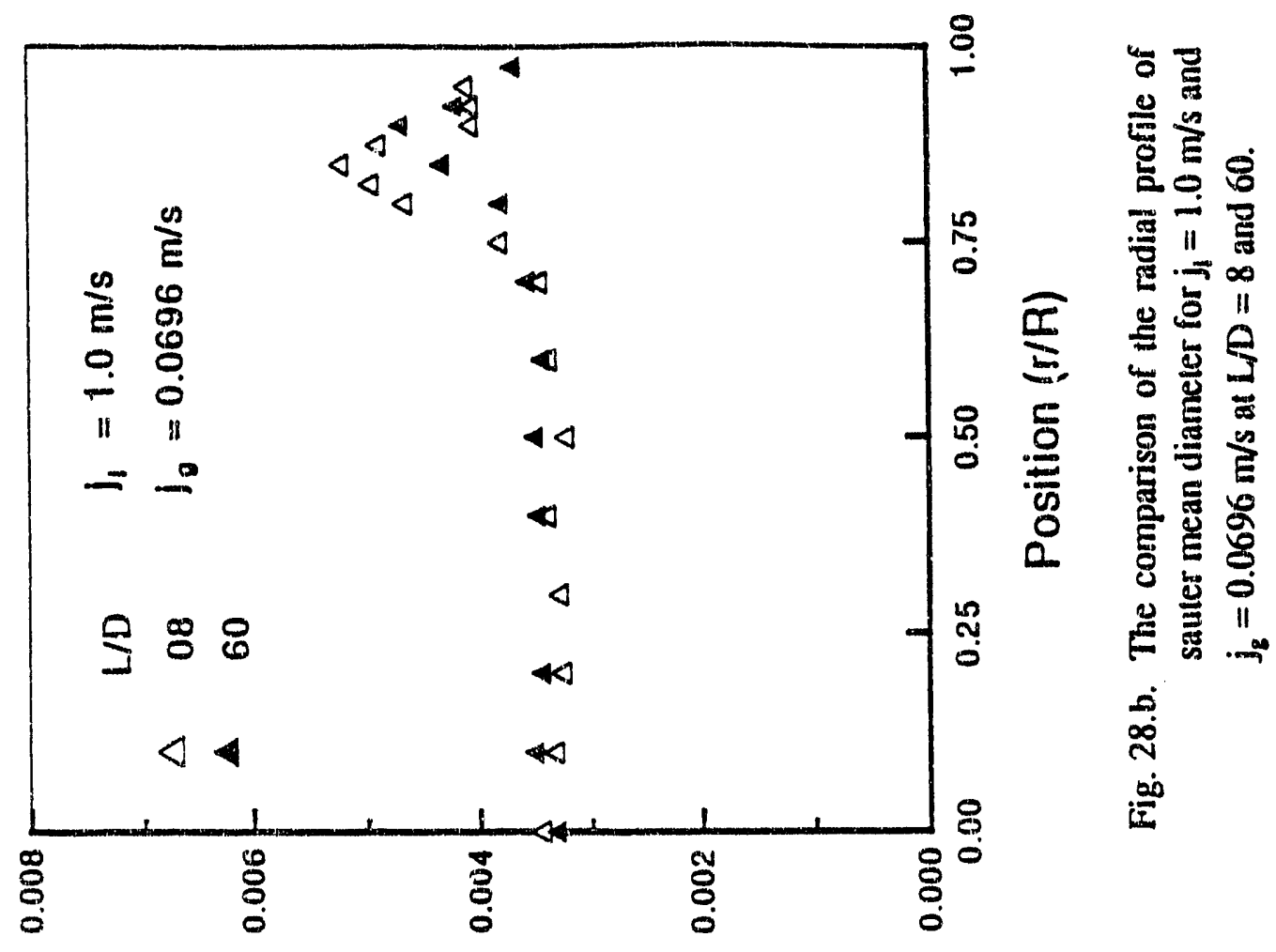

(u) JəłəUE! U UE⿰W Jaines

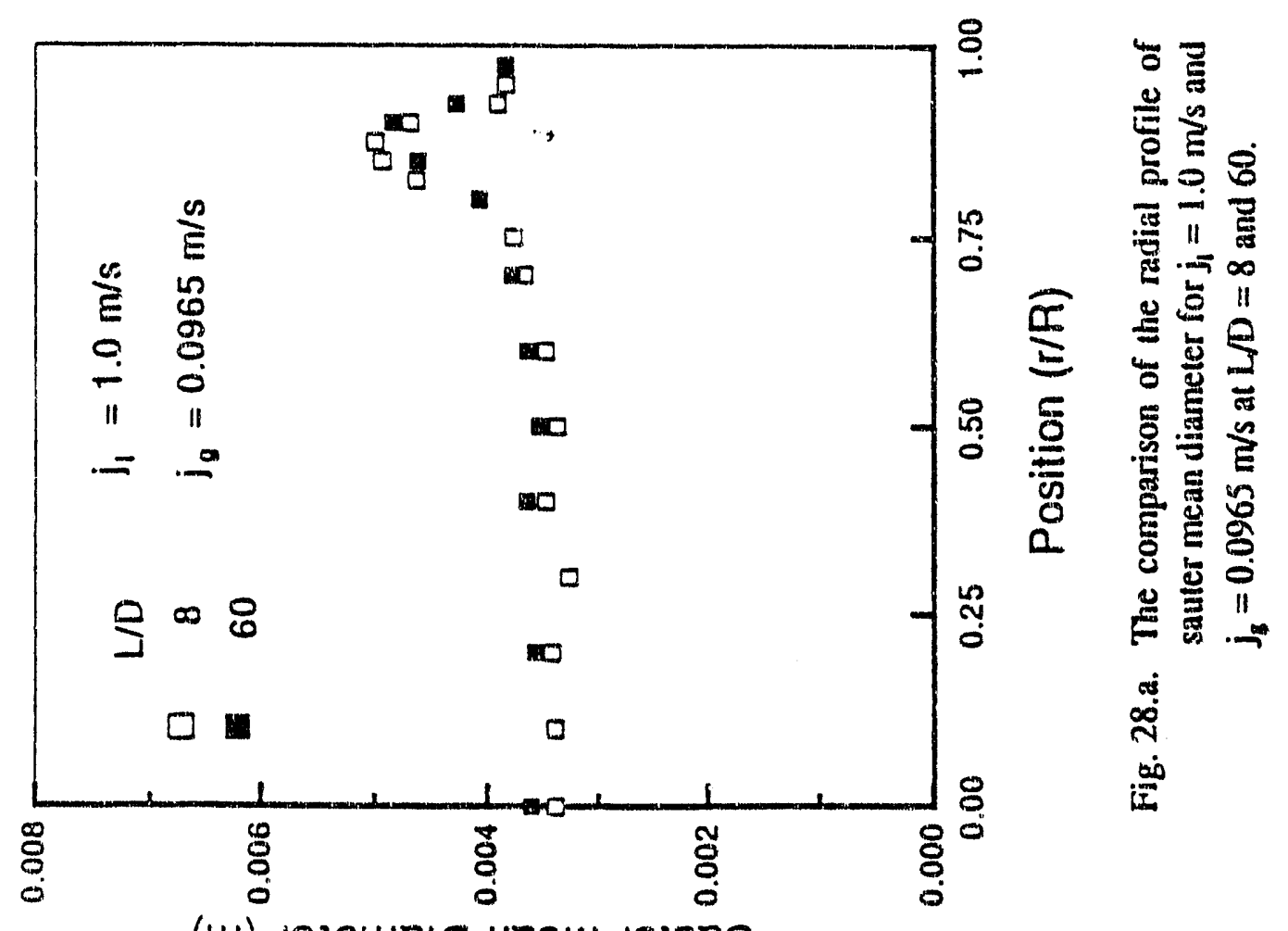

(w) dəןaur! u ueaw jatnes 


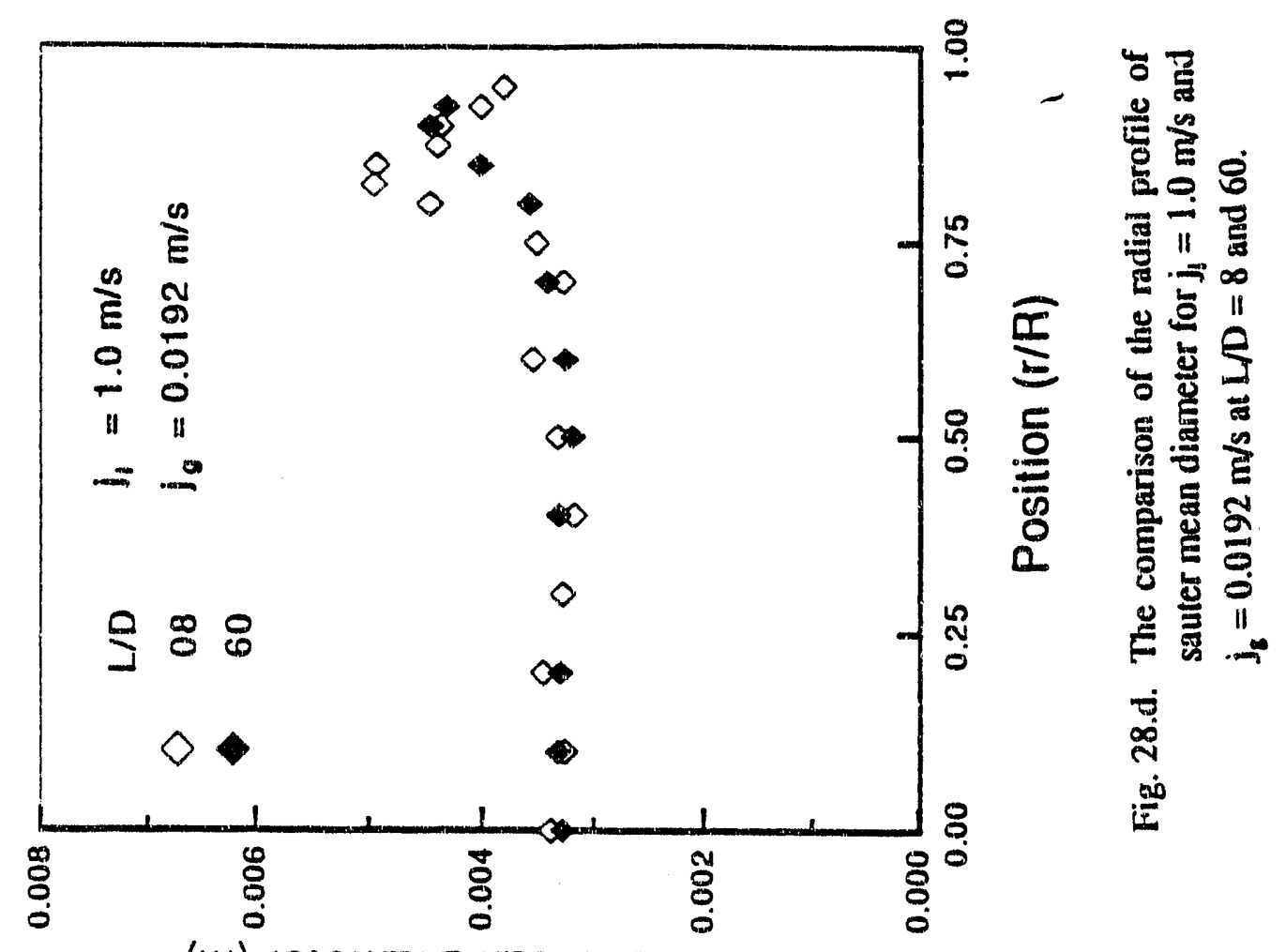

(u) dajaue! u ueaw jeines

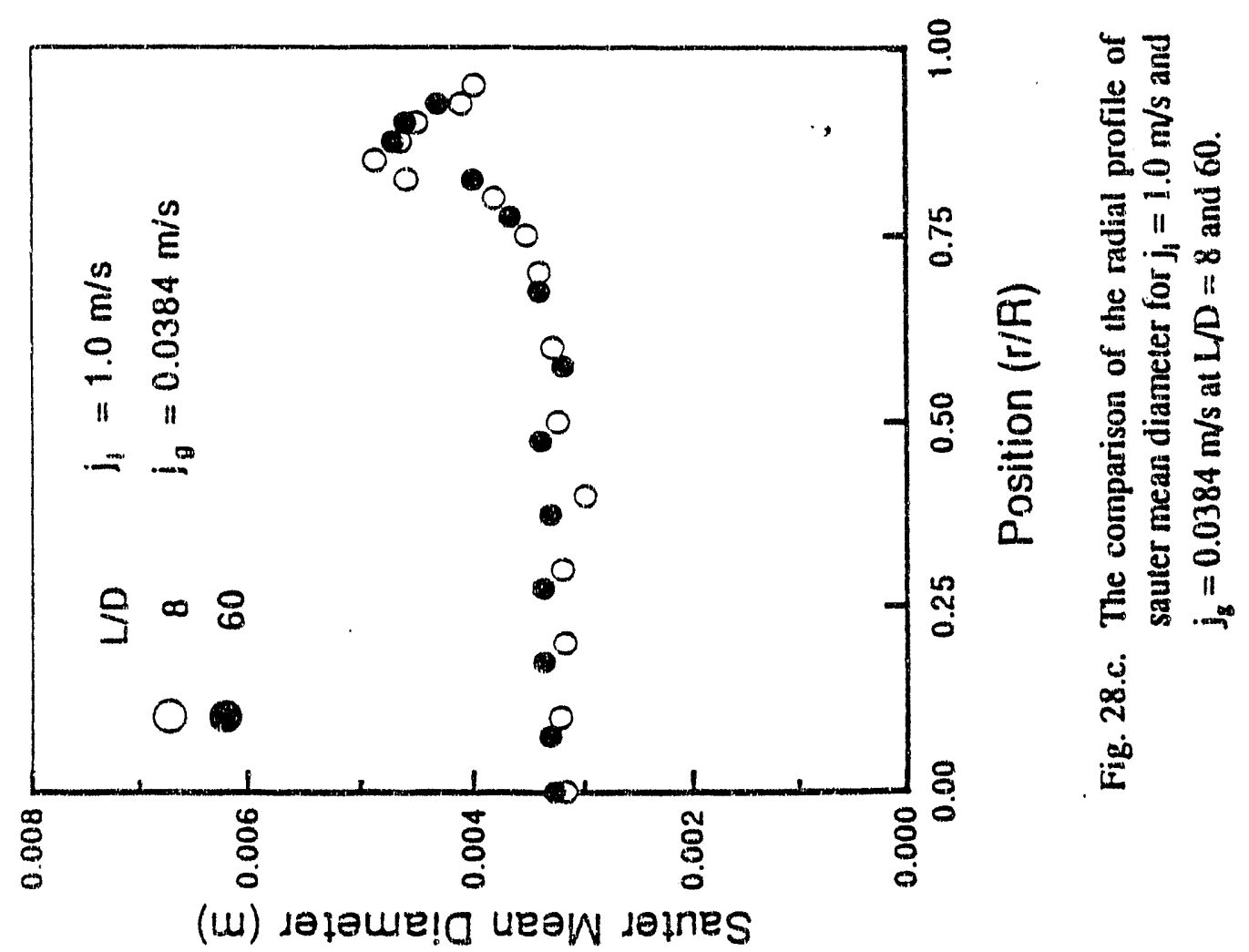



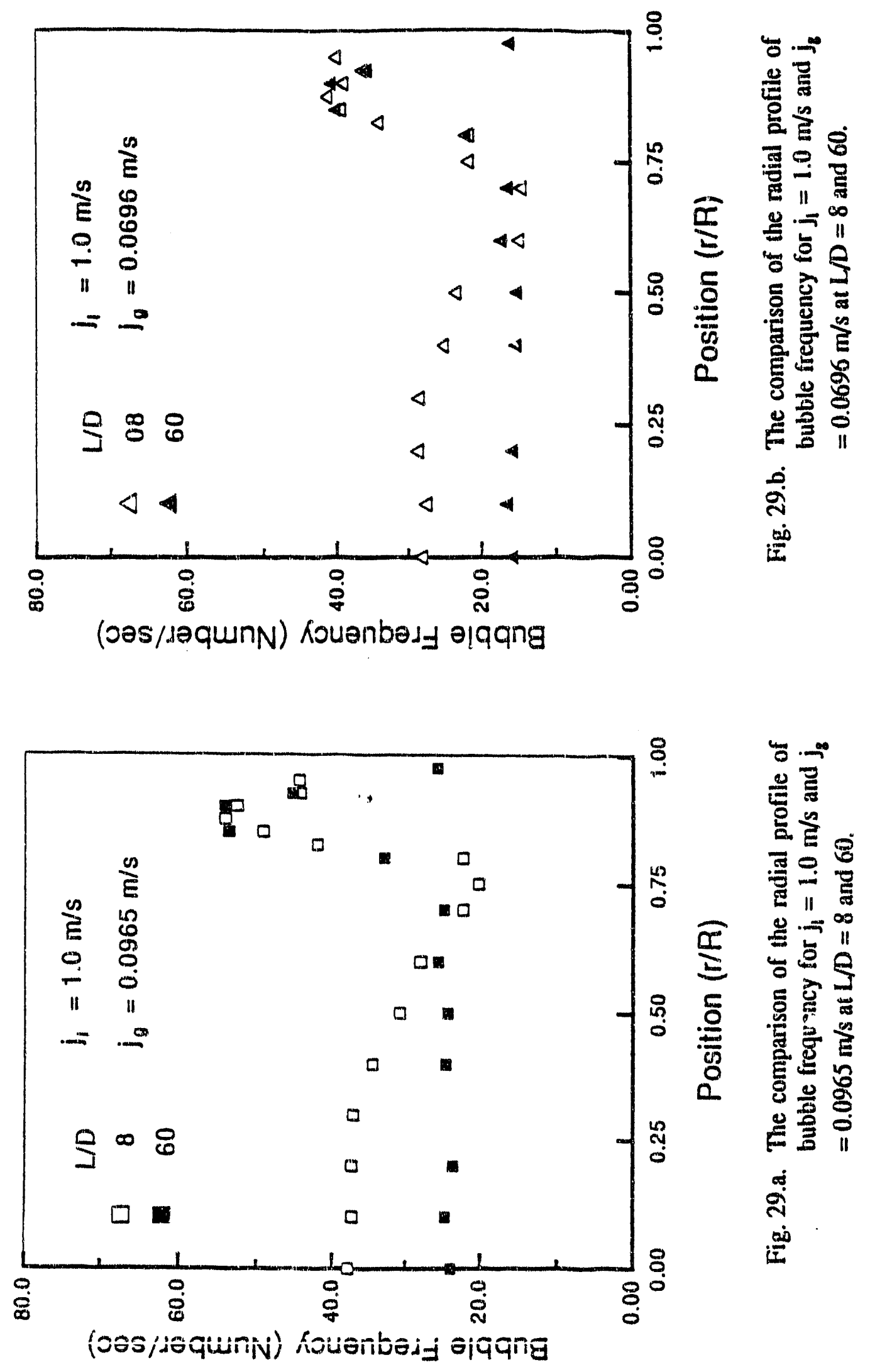

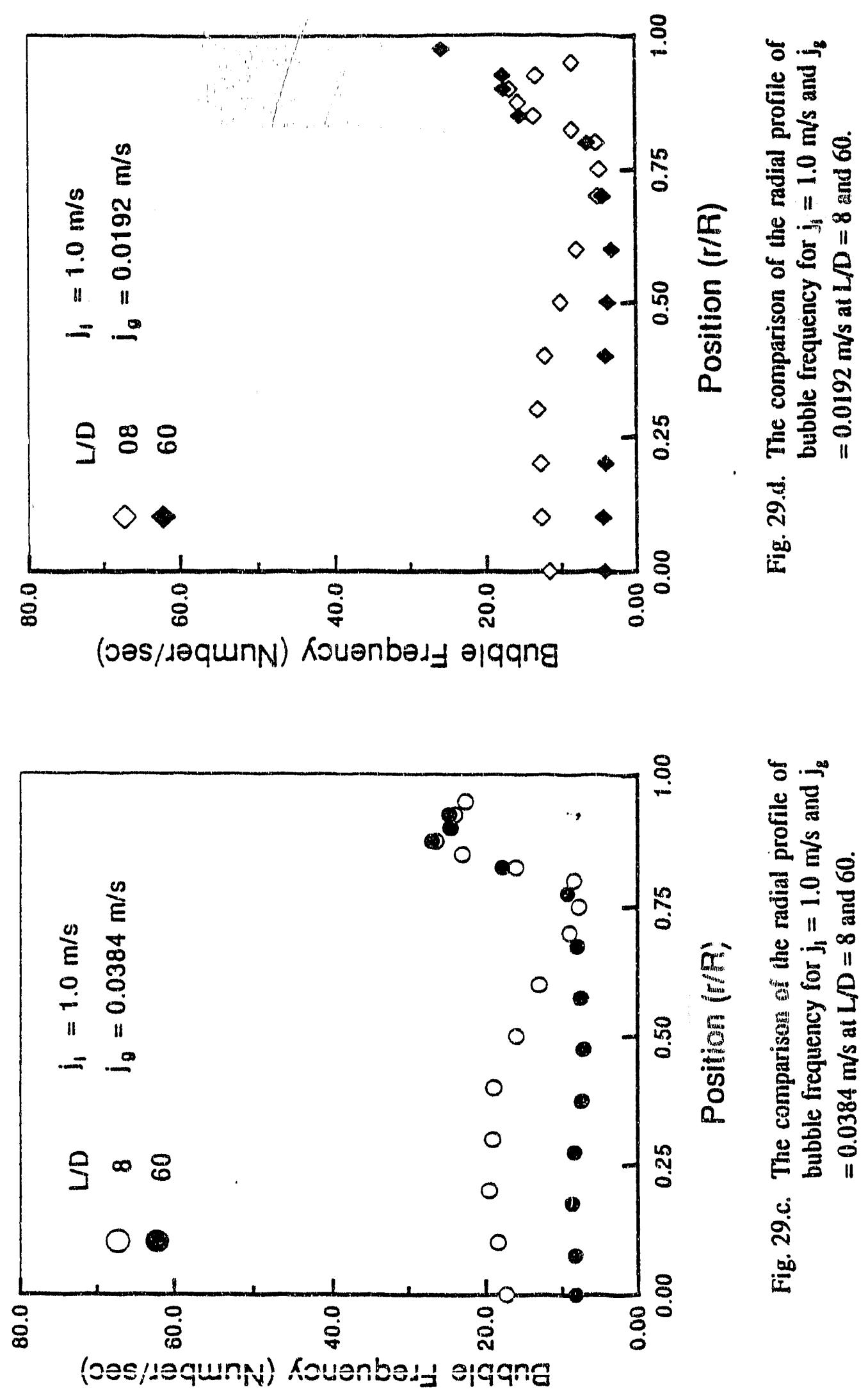

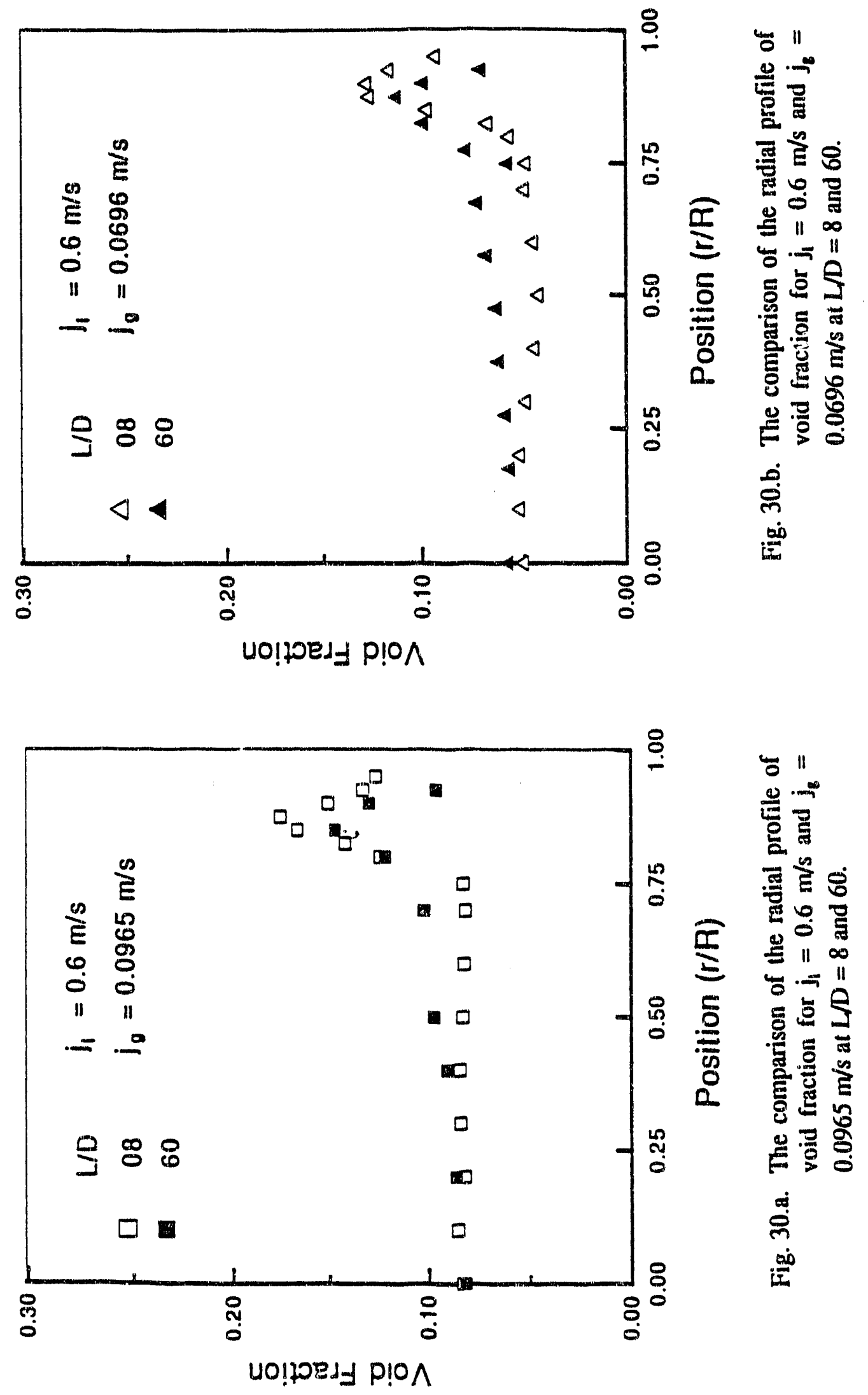

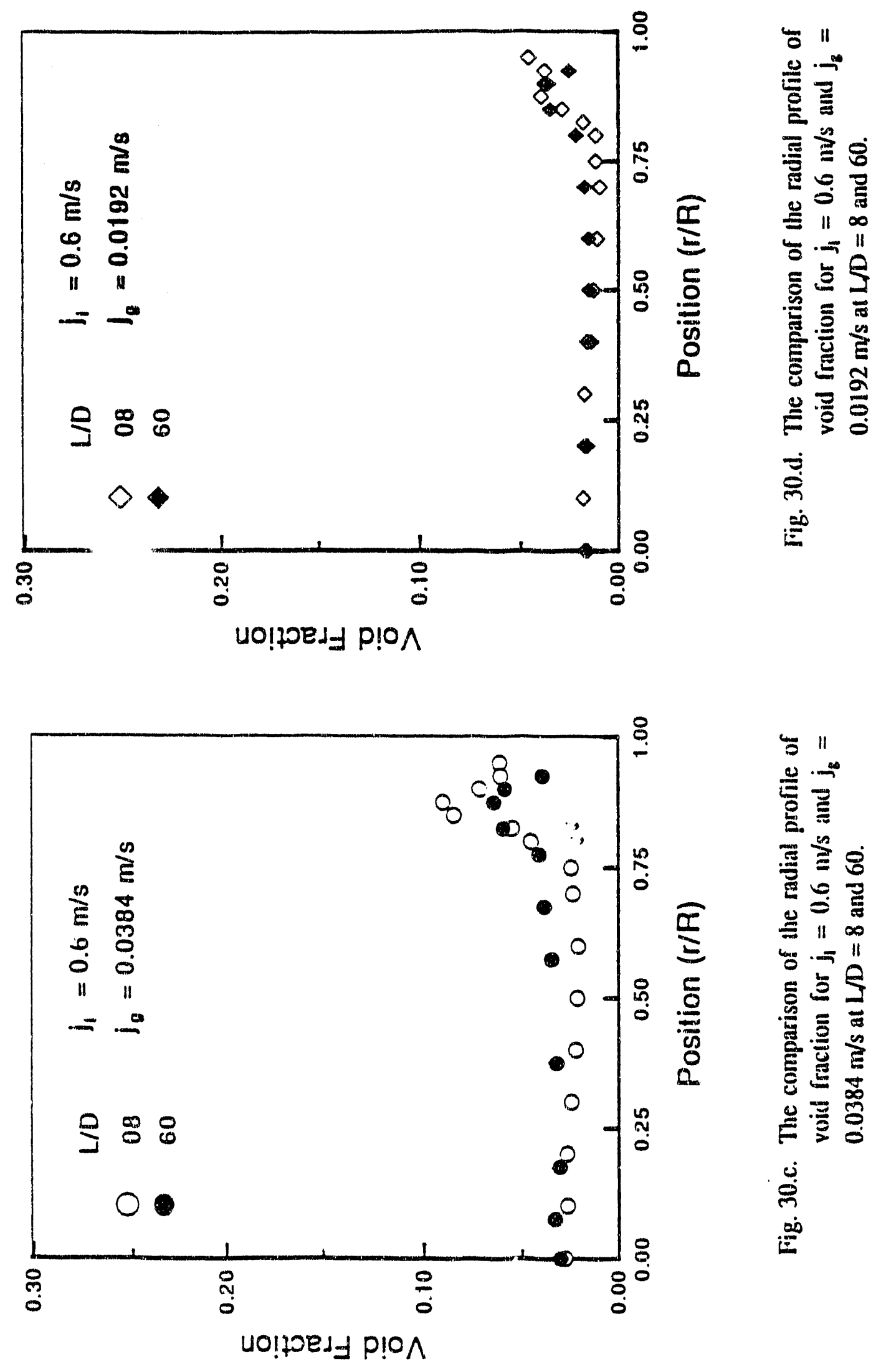

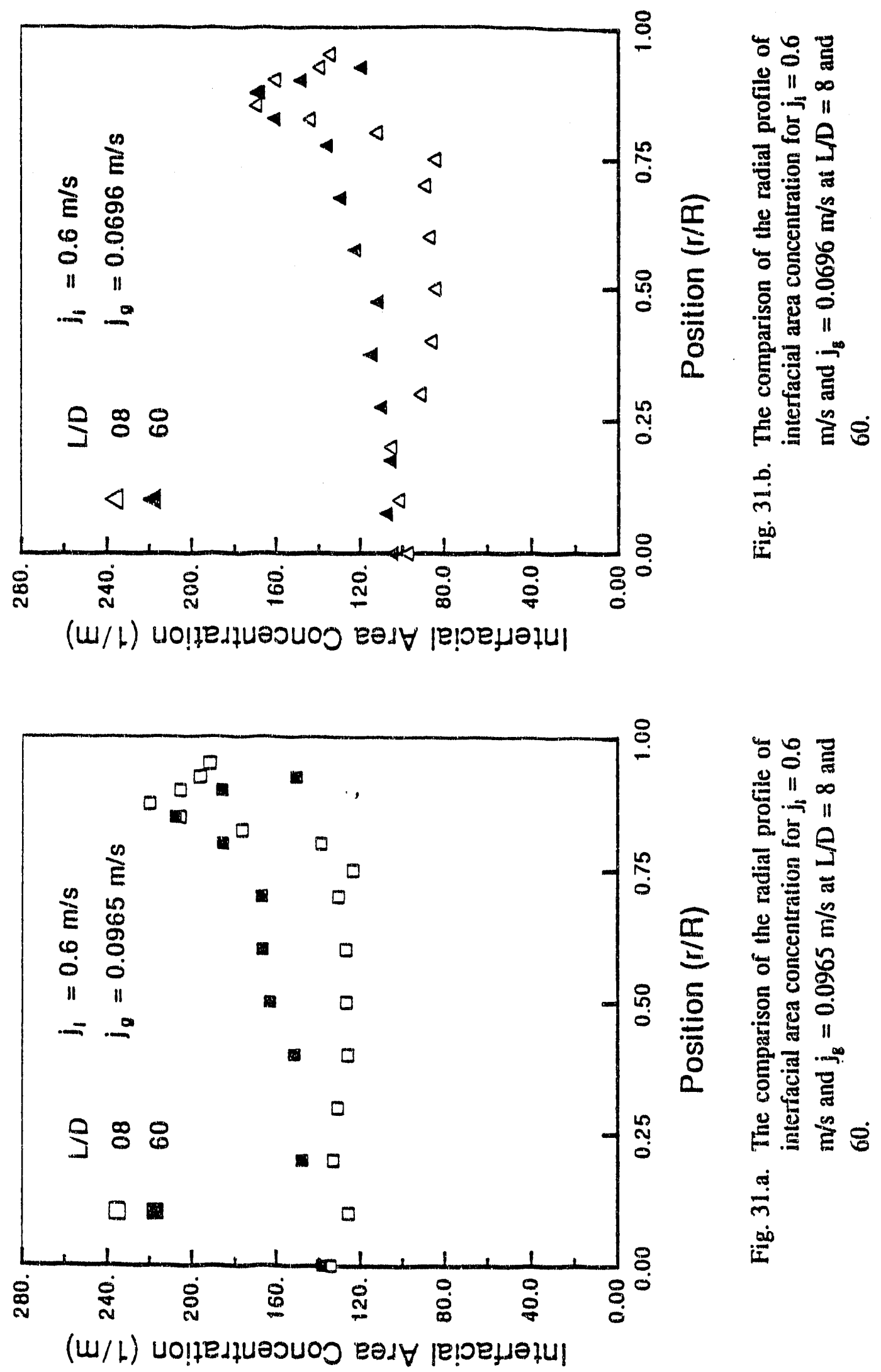

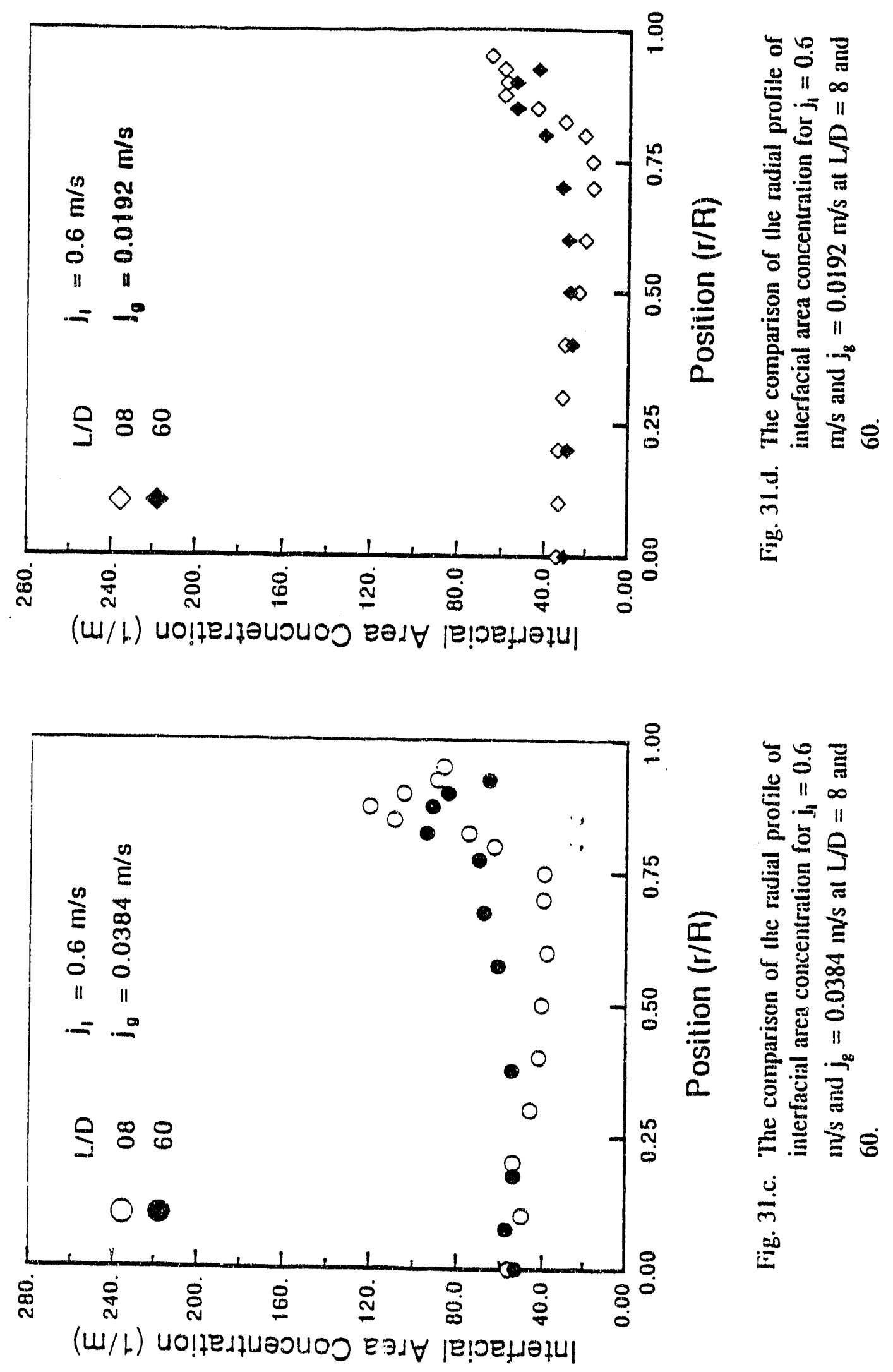

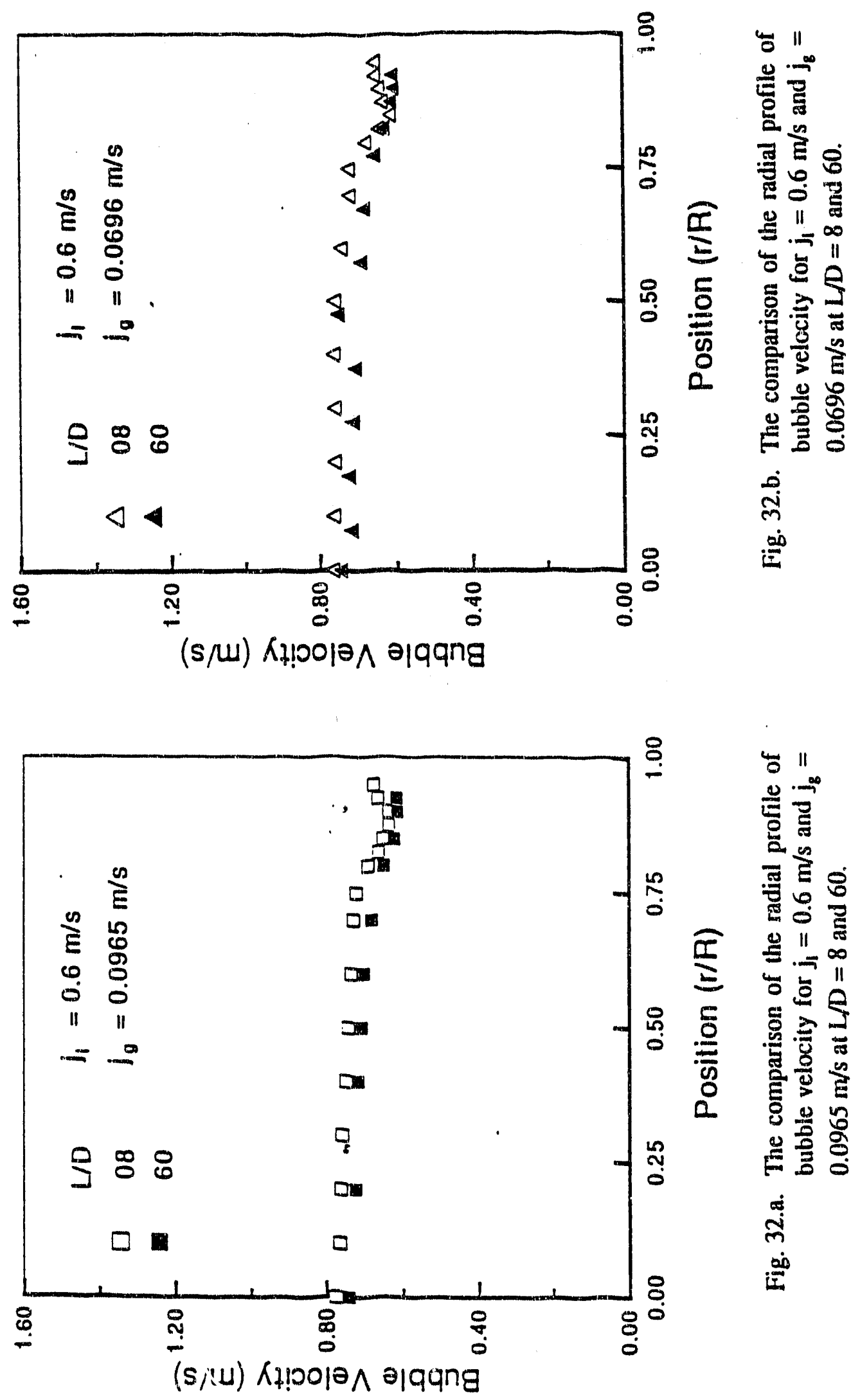

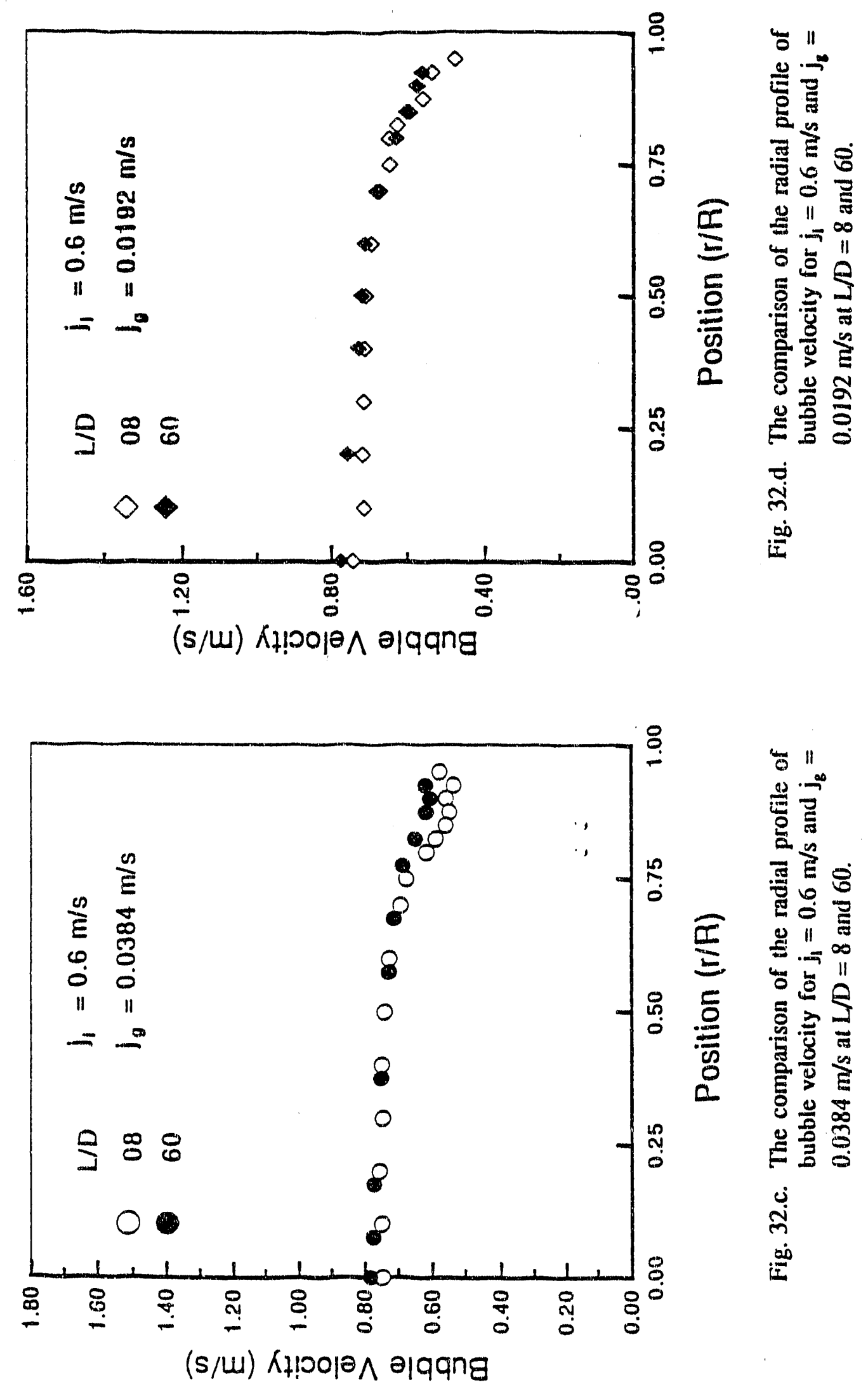

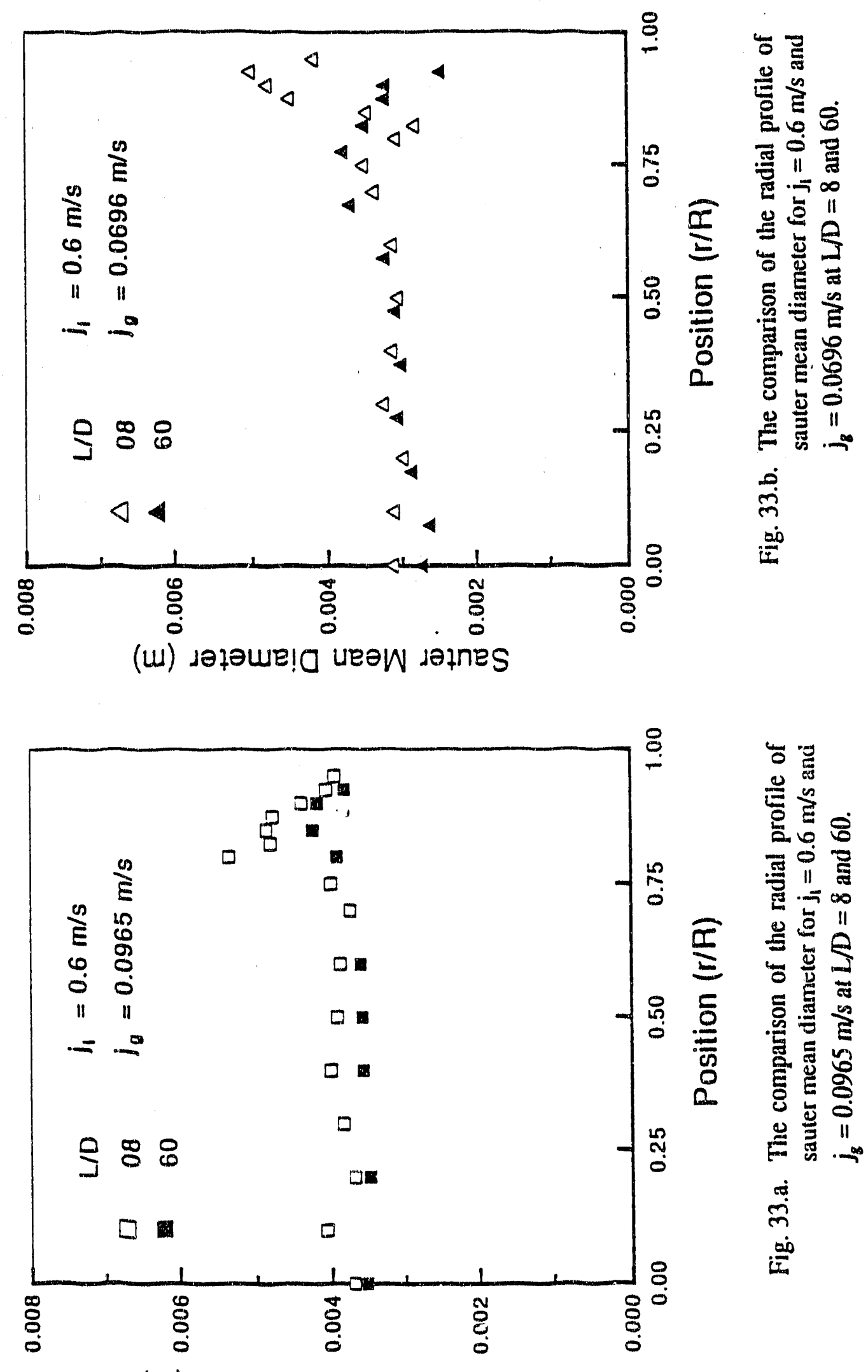

(w) jojause! u ueəw jałnes 

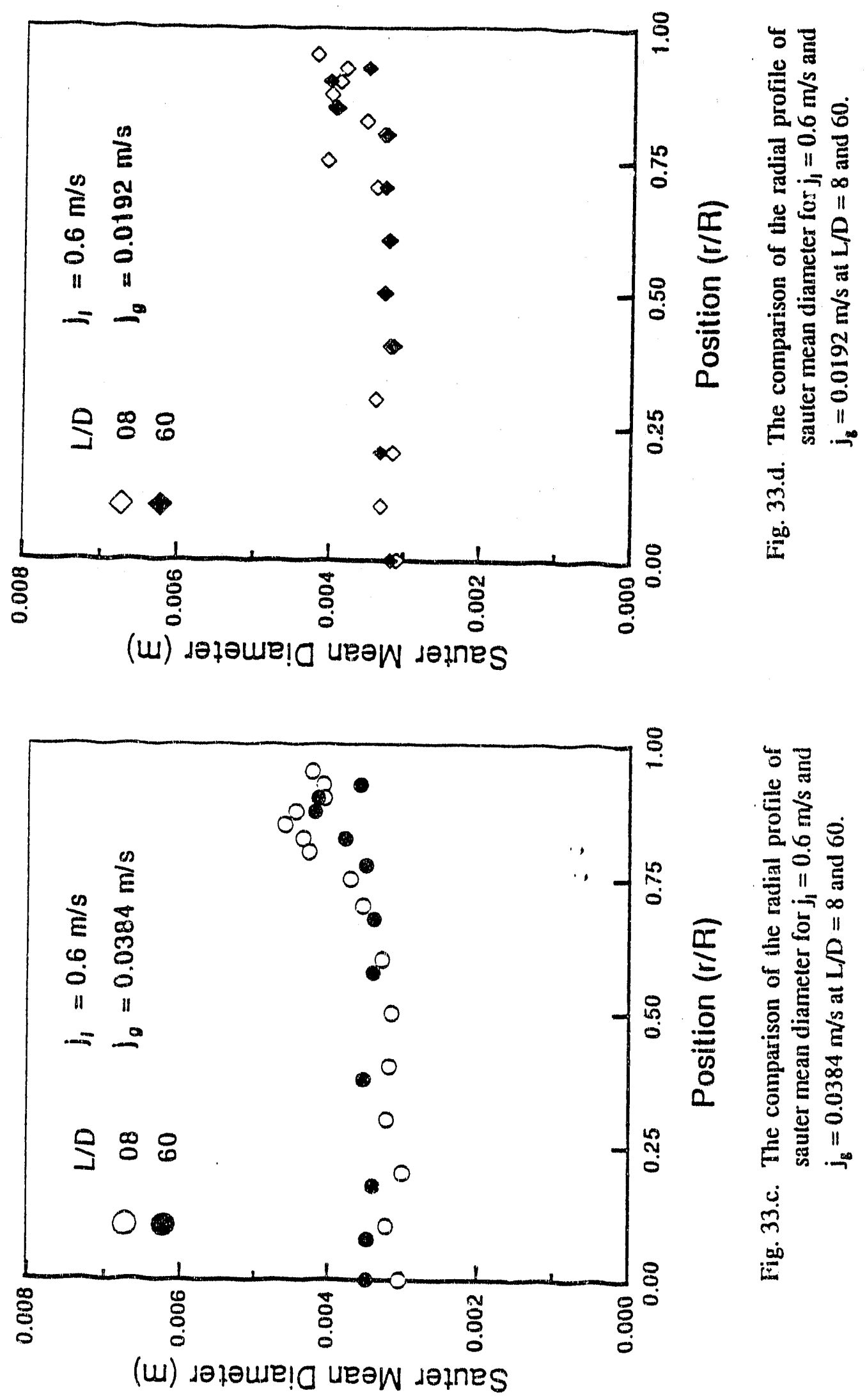

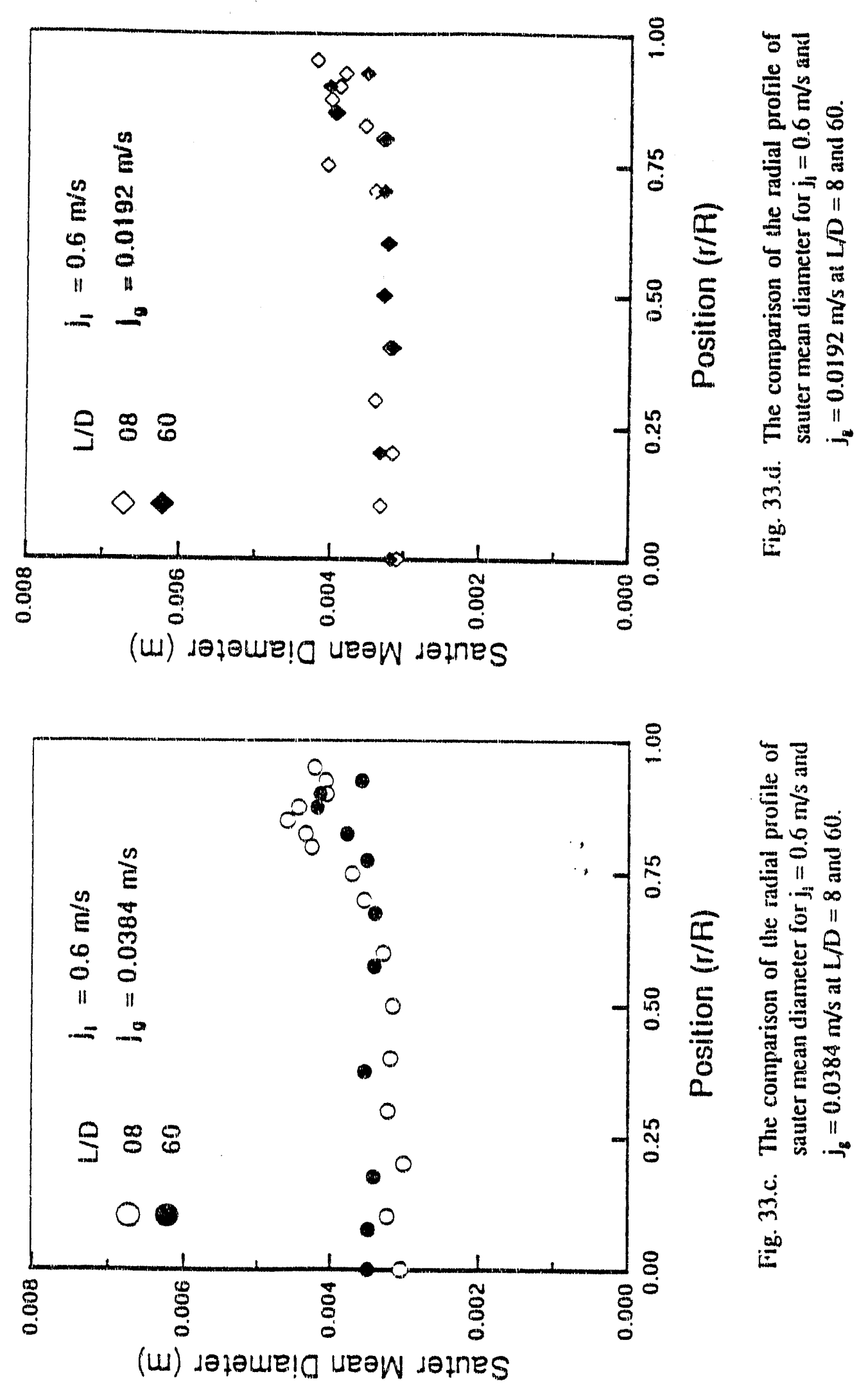

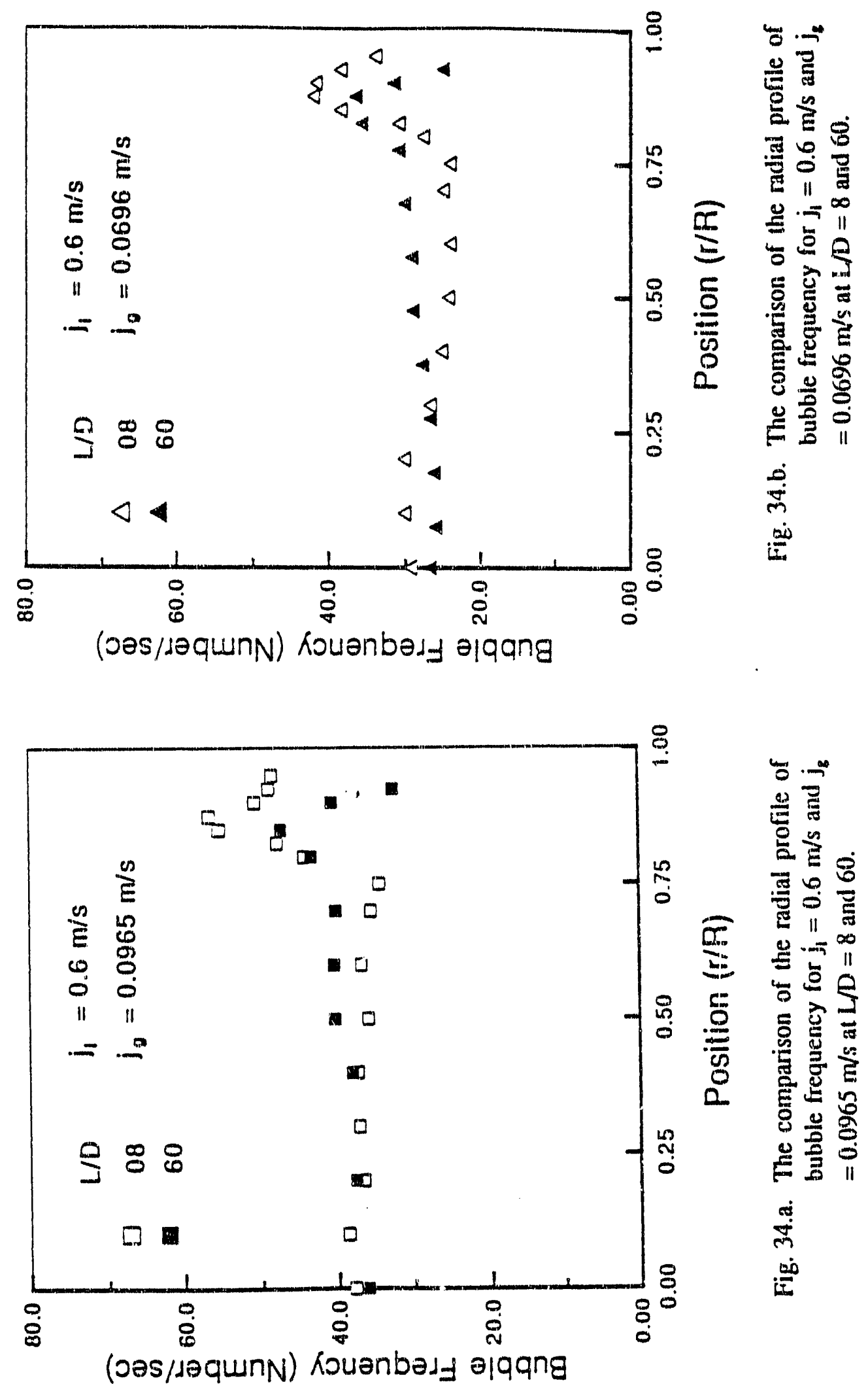

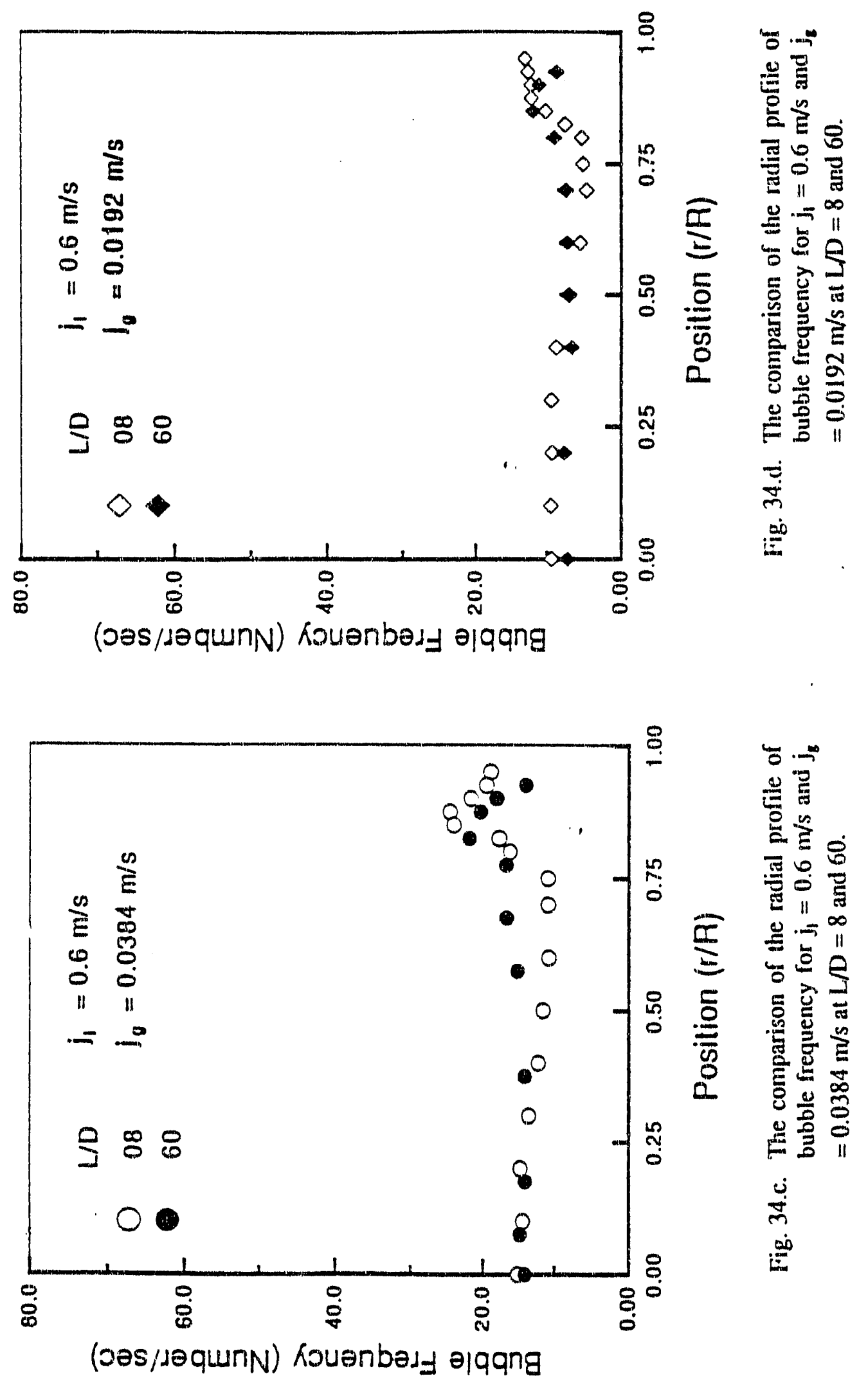

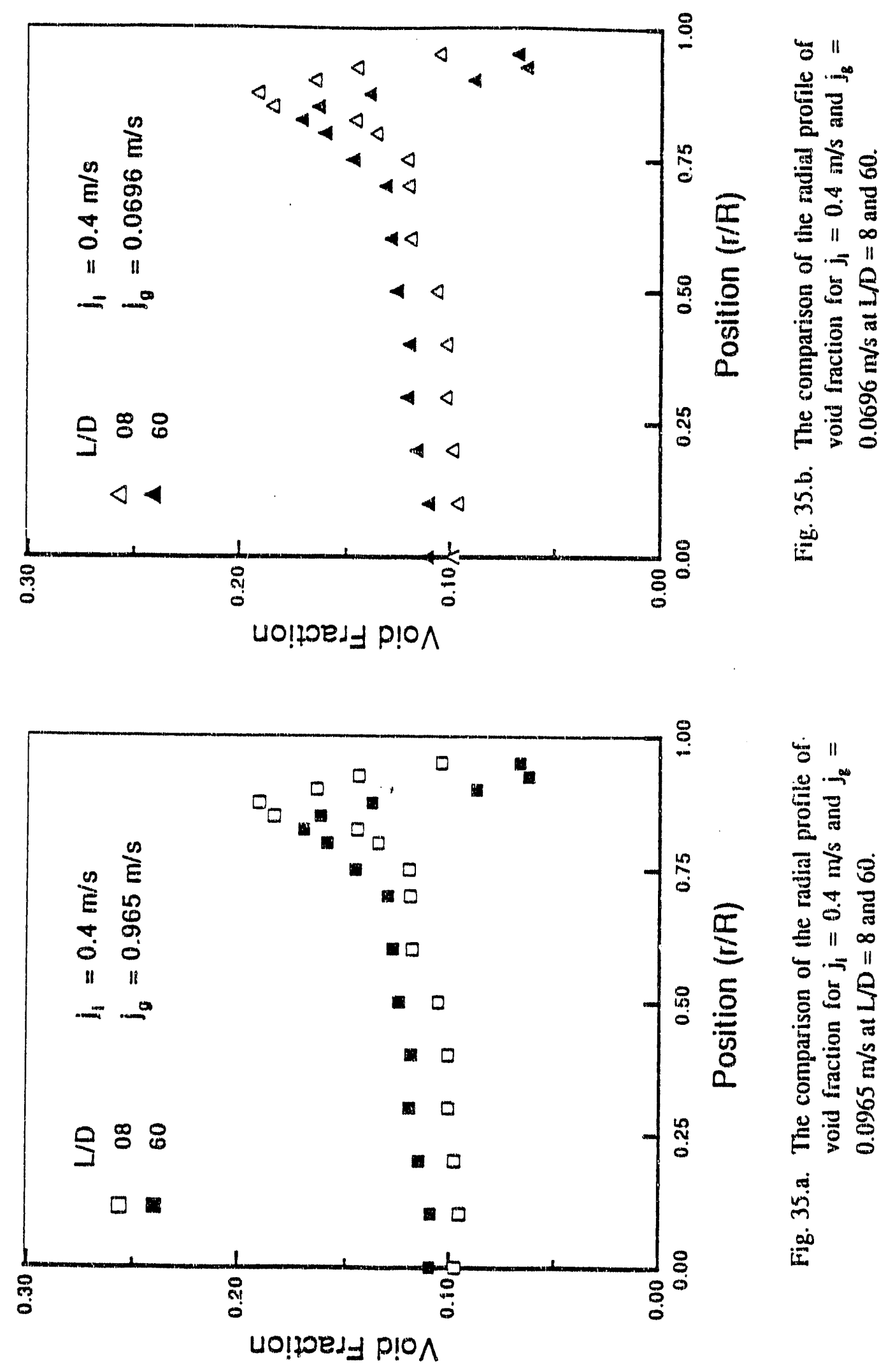

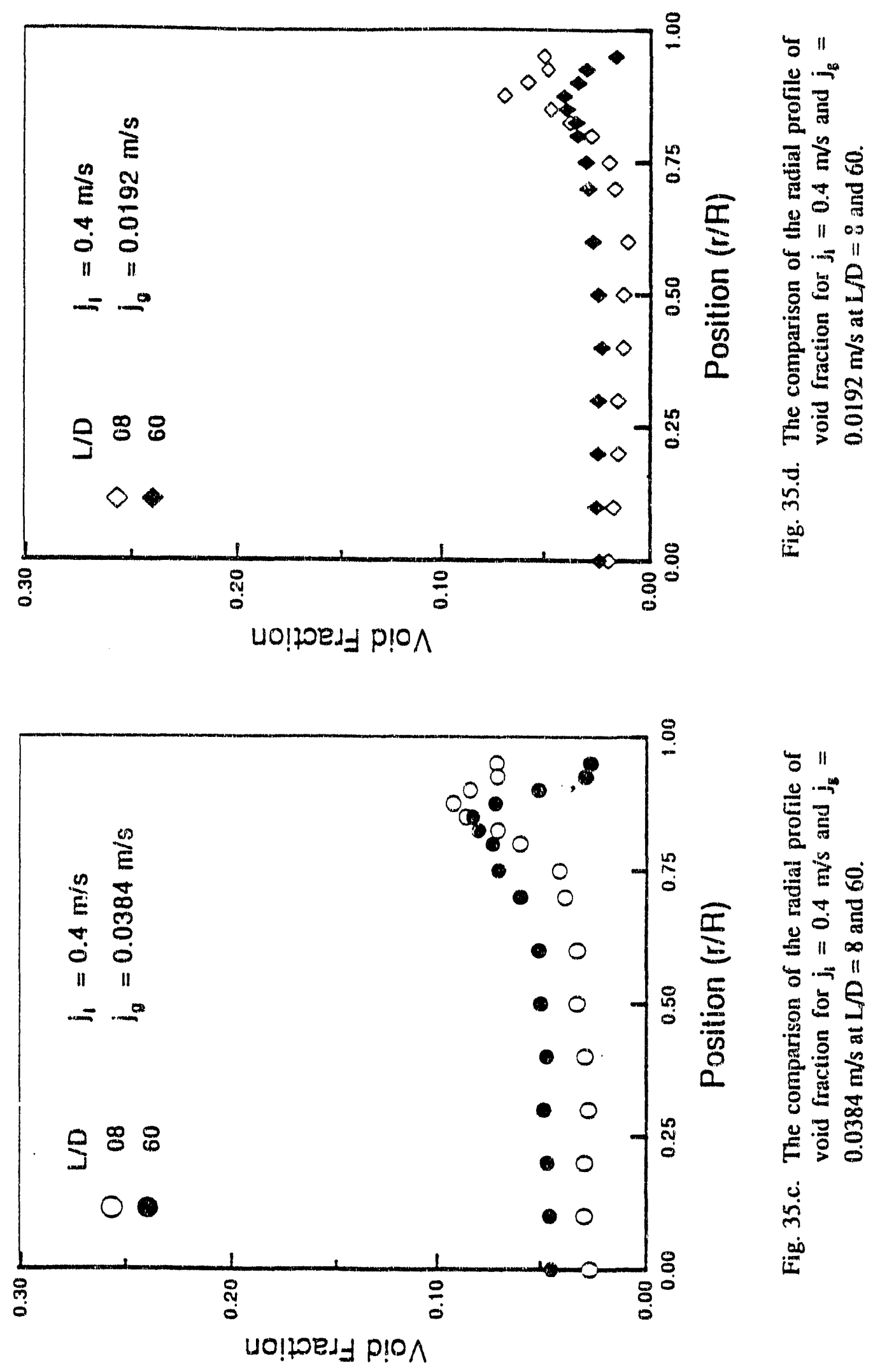

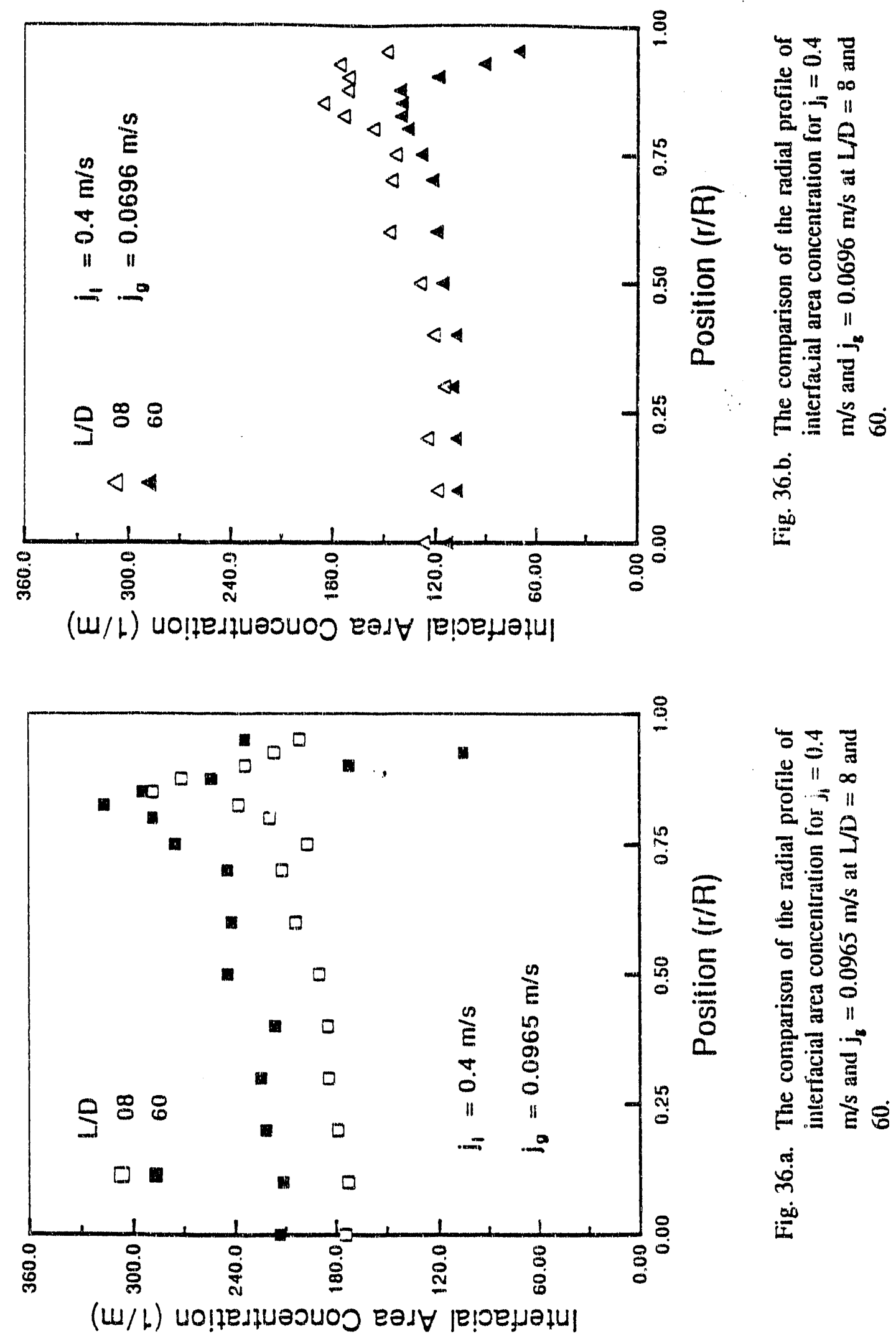

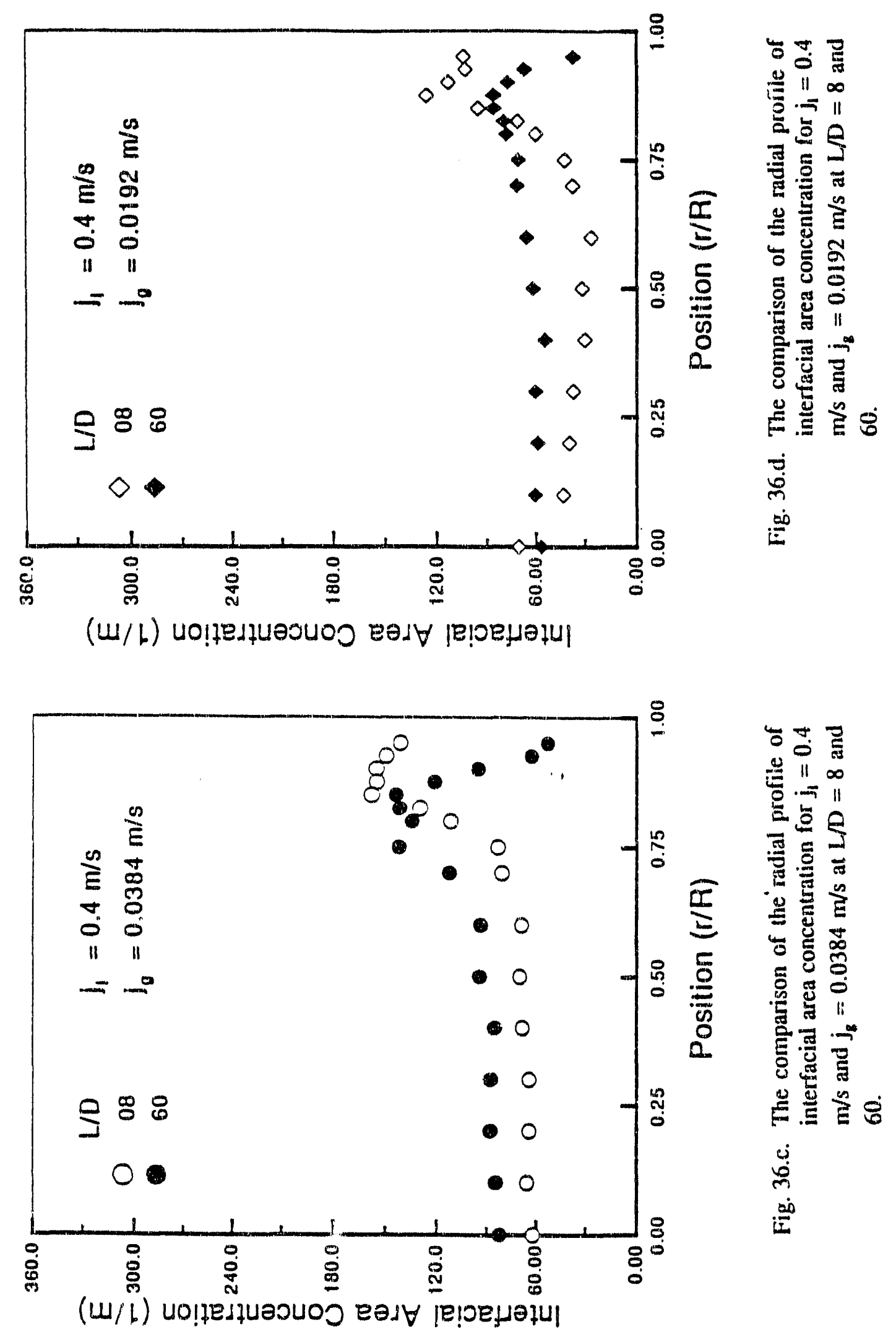

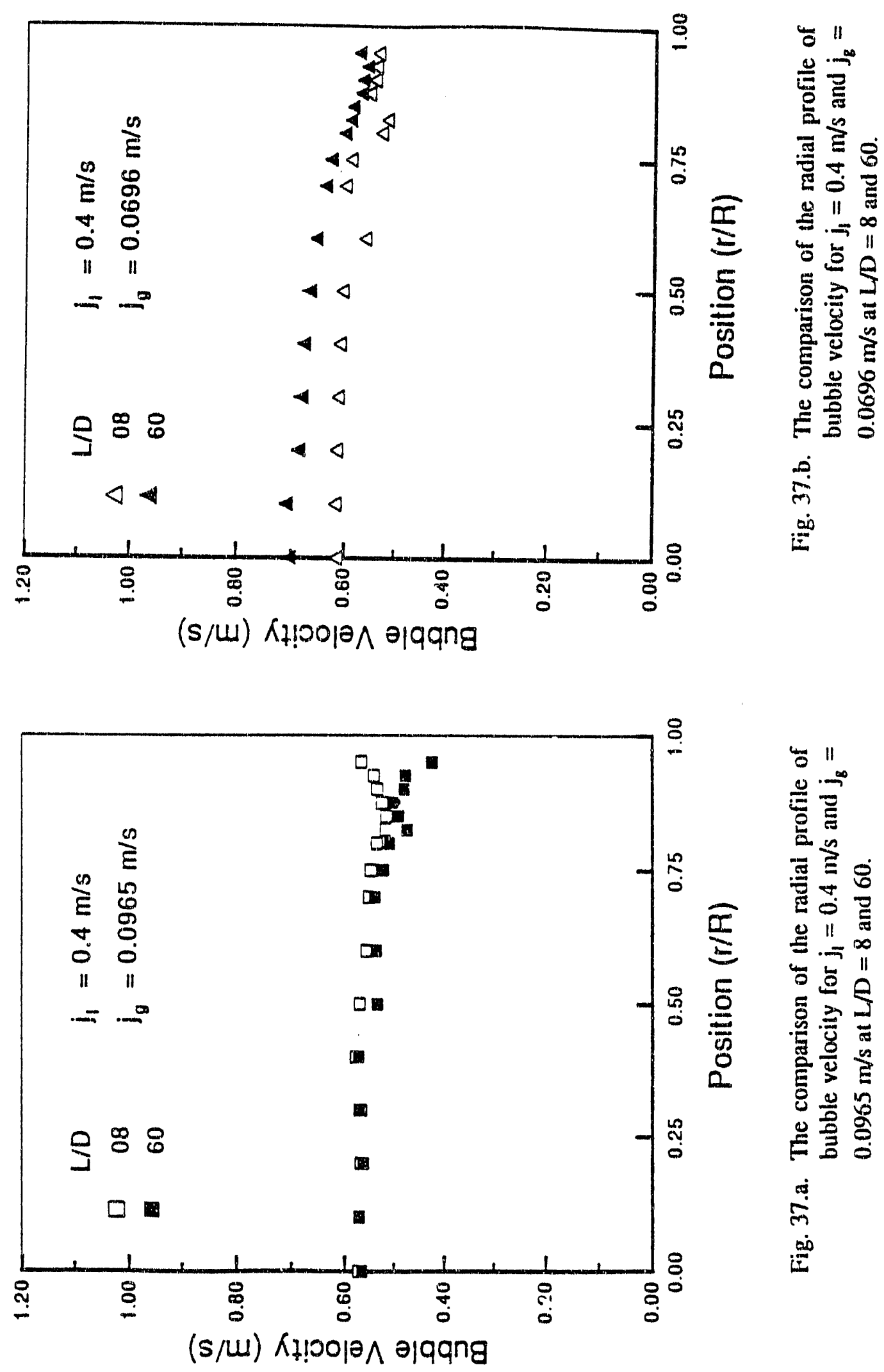

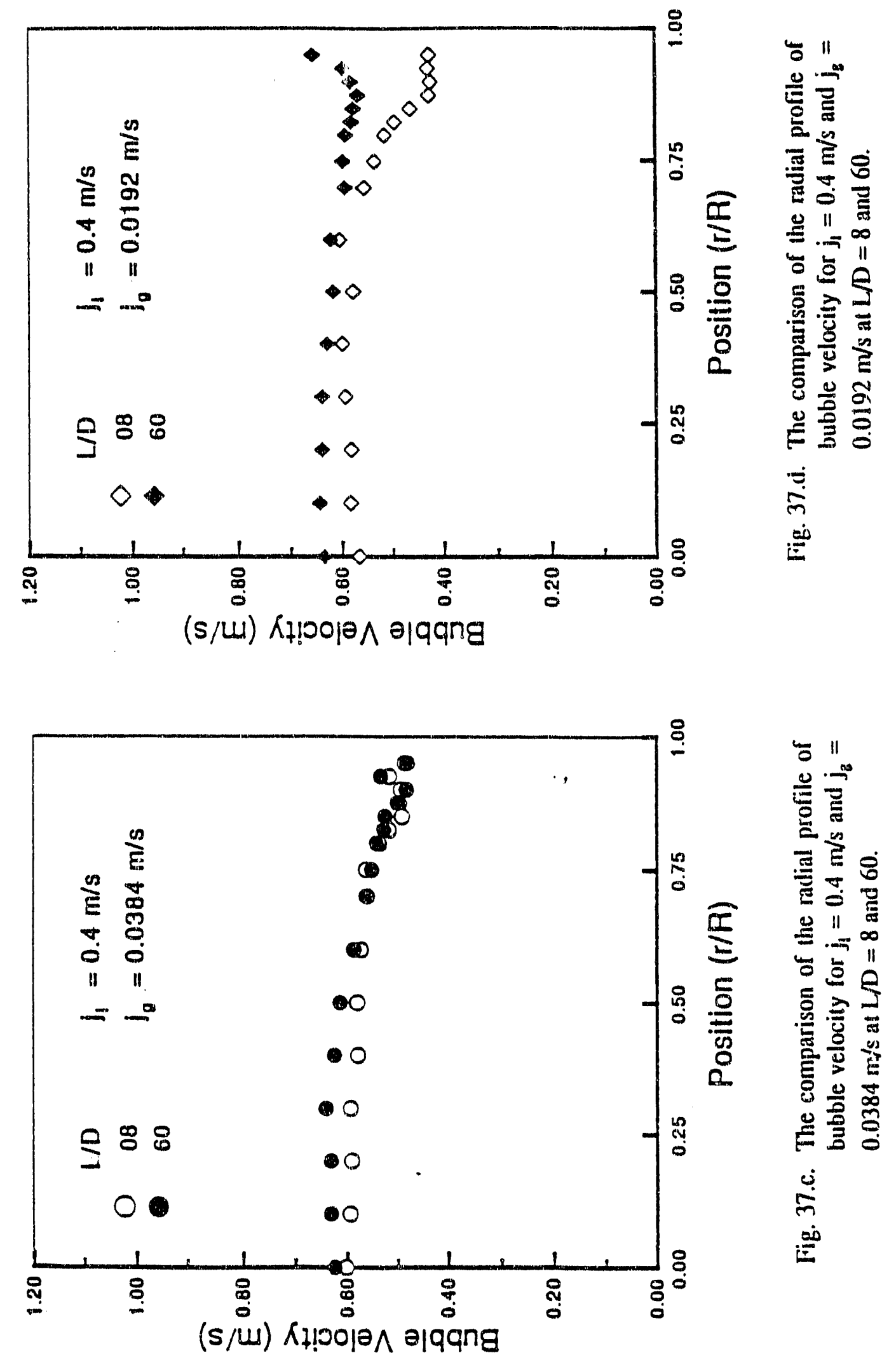

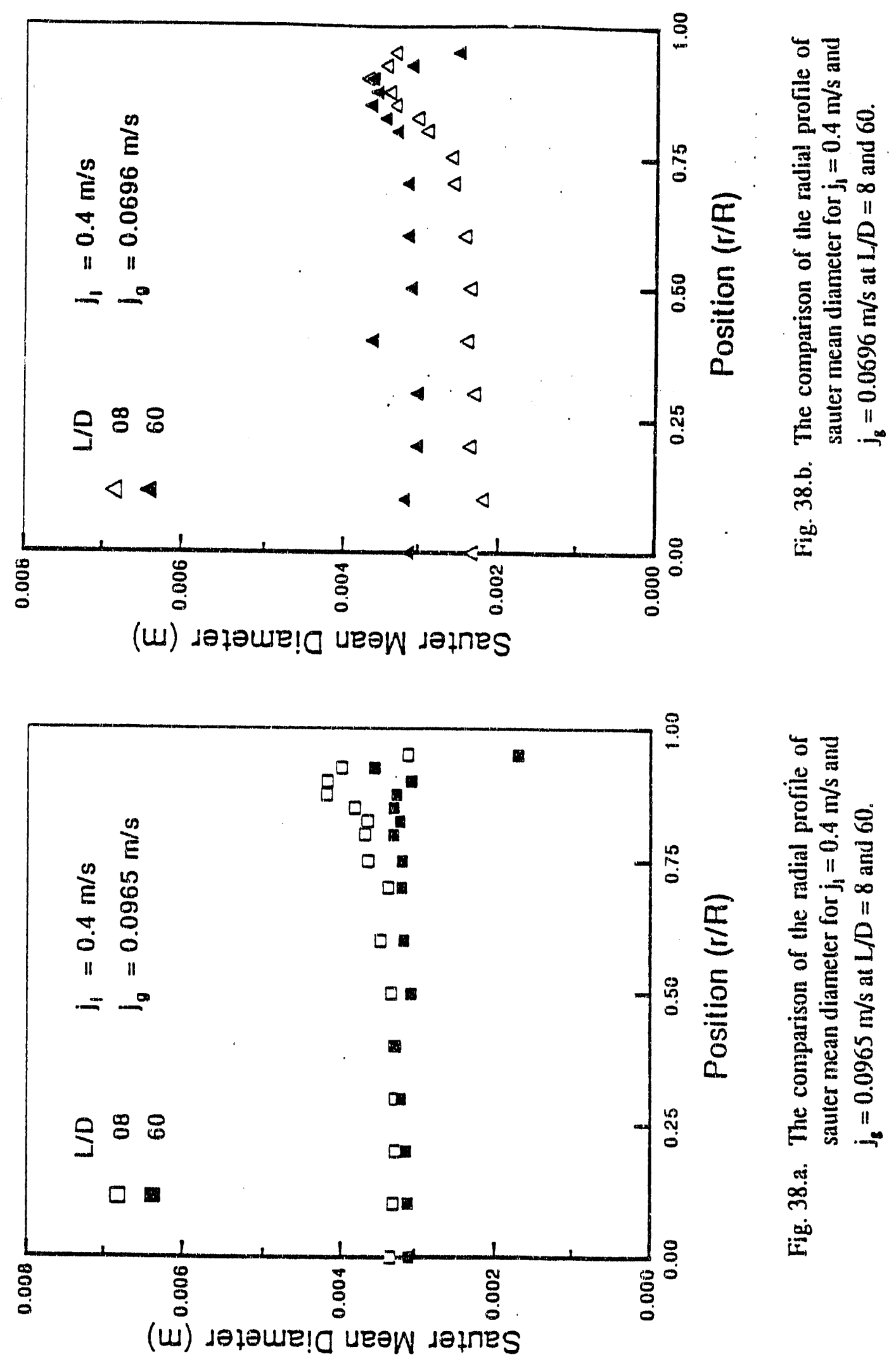

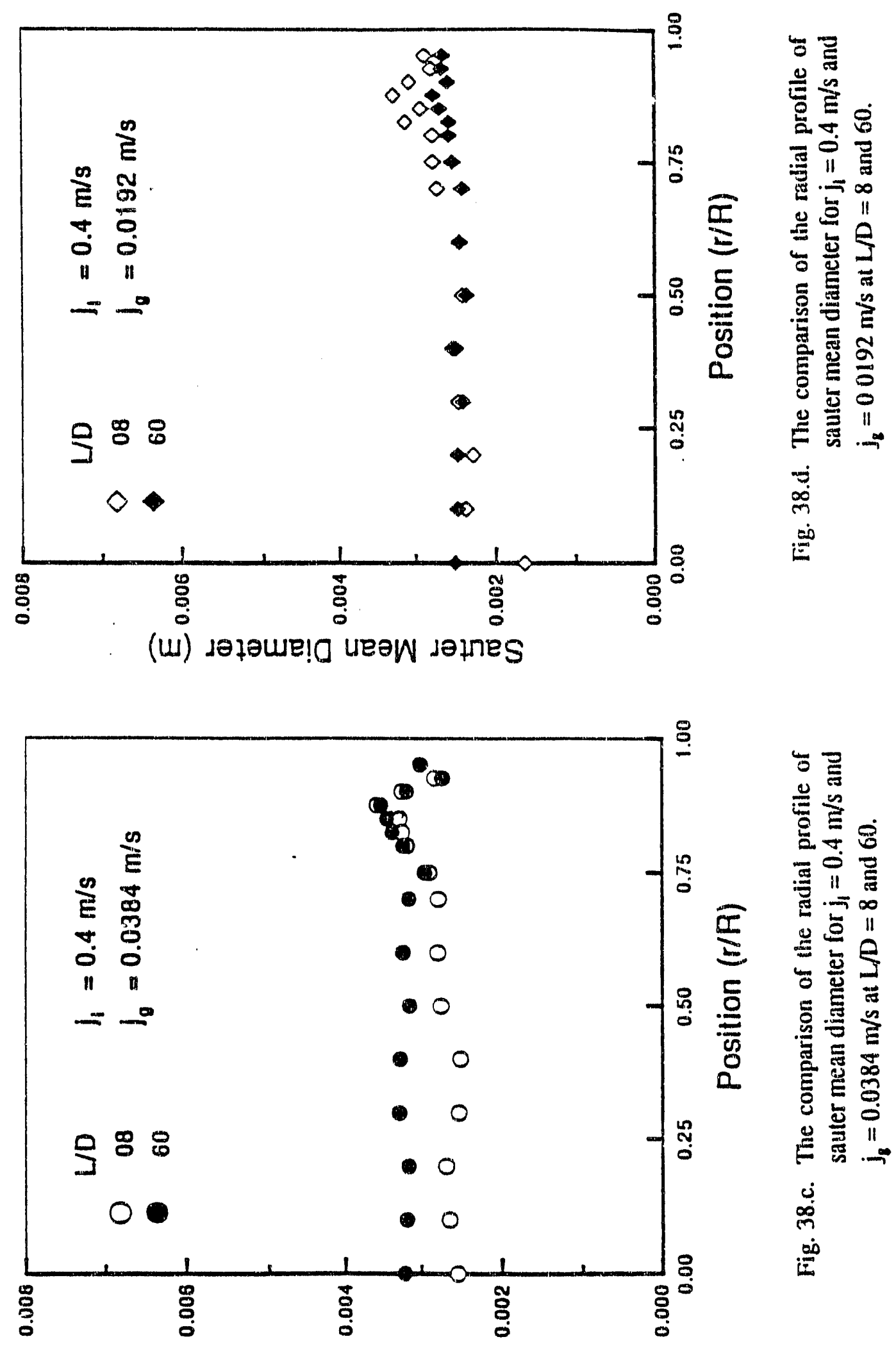

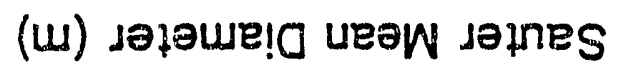



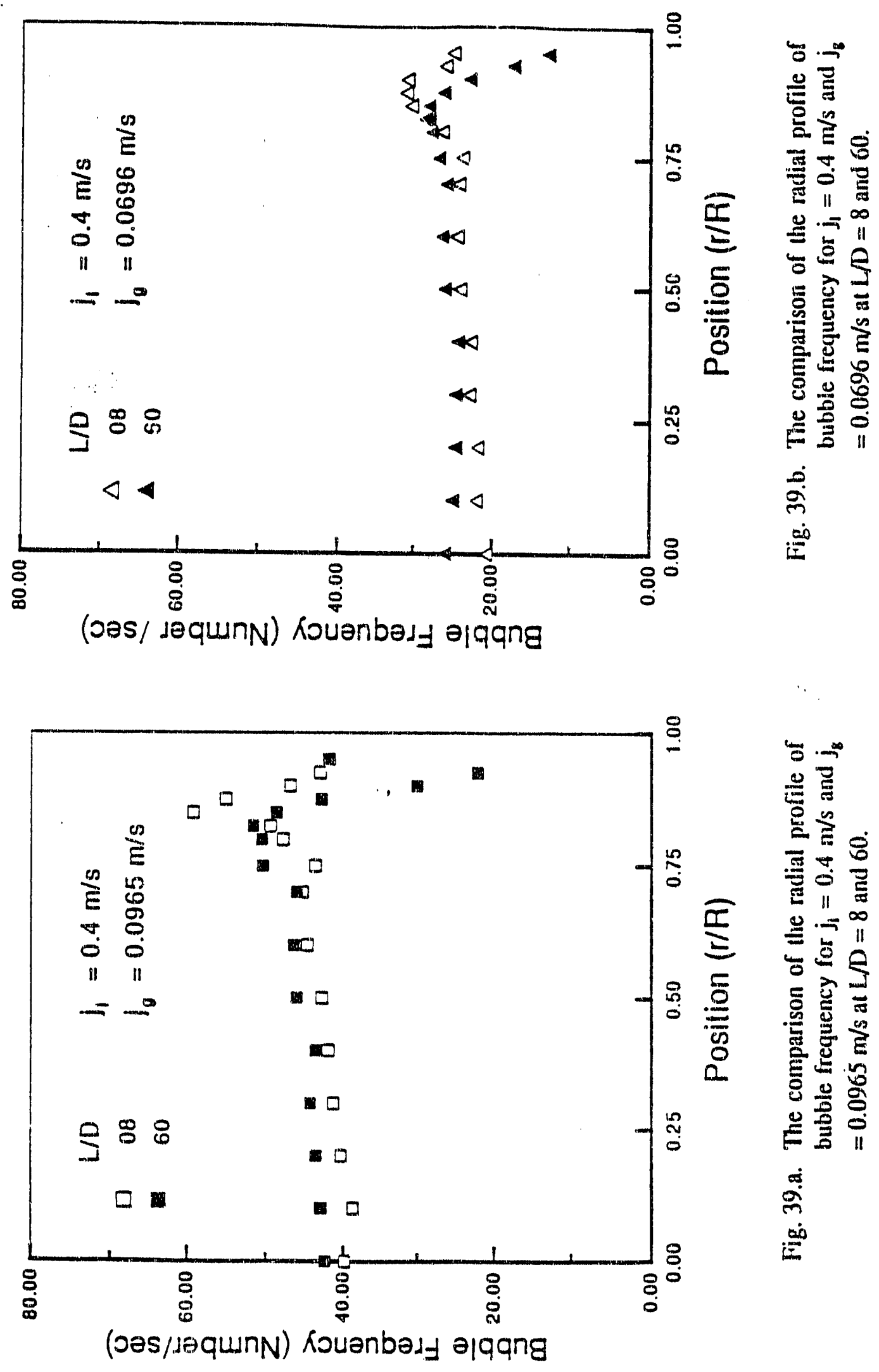

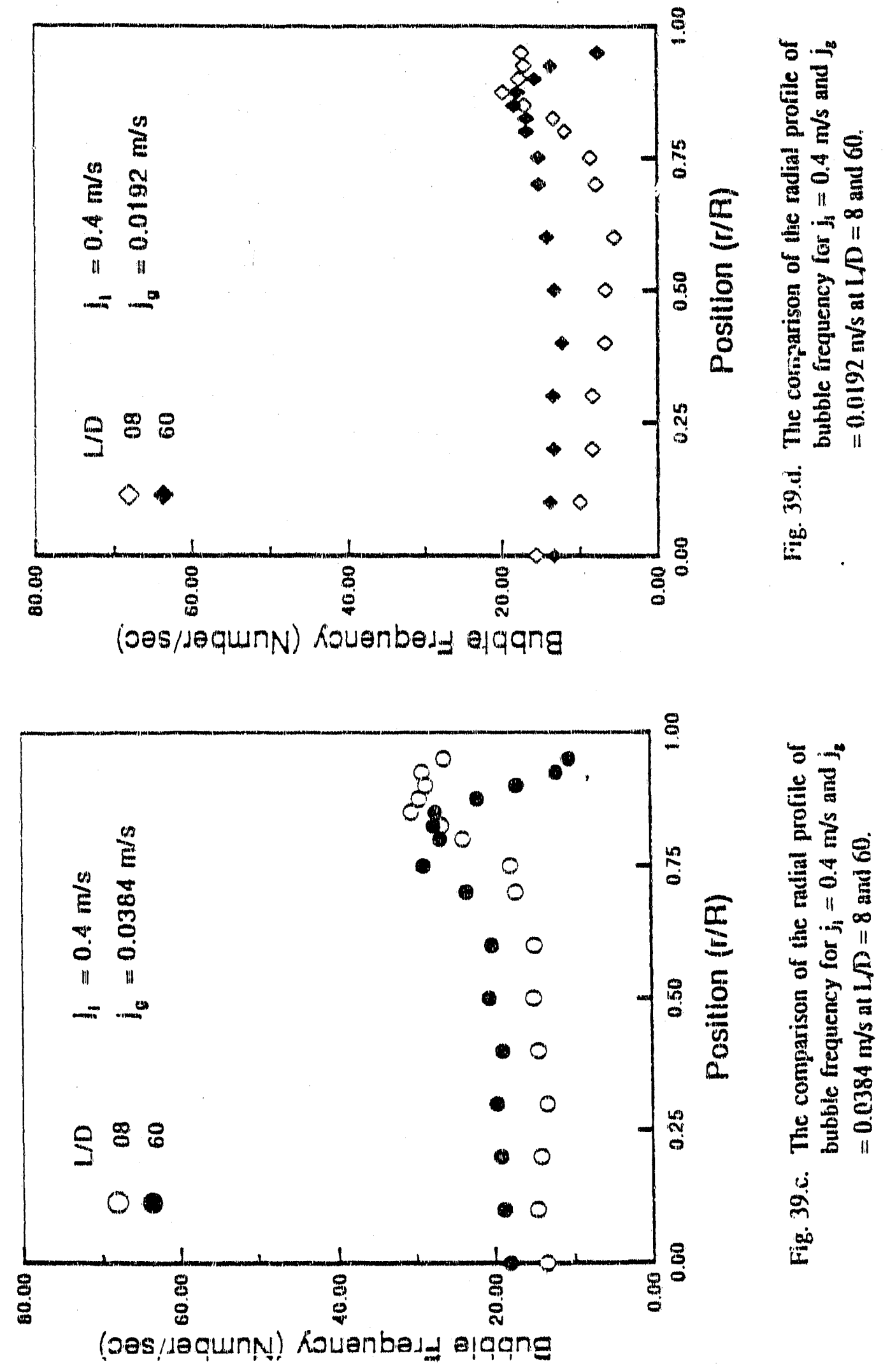

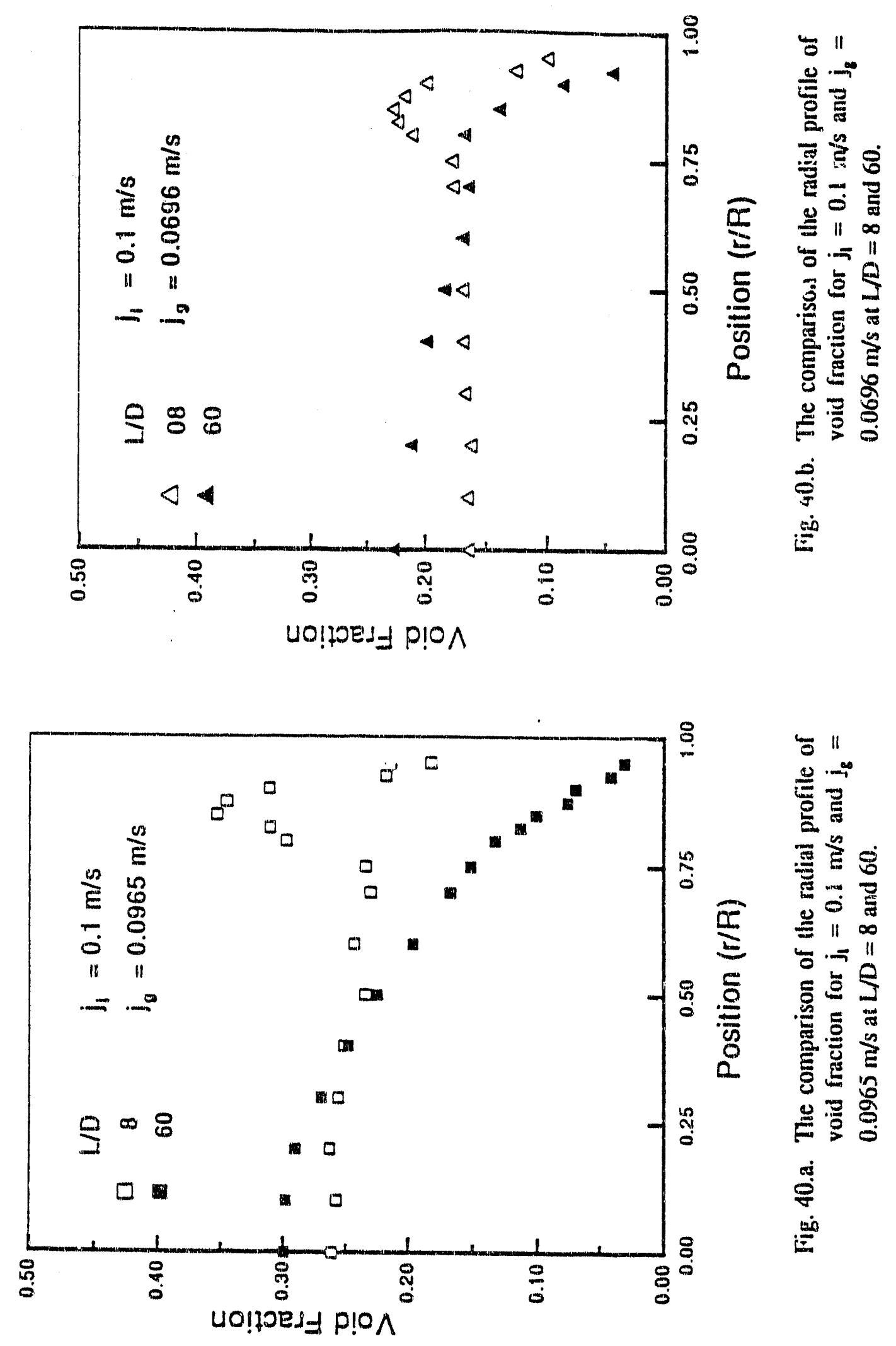

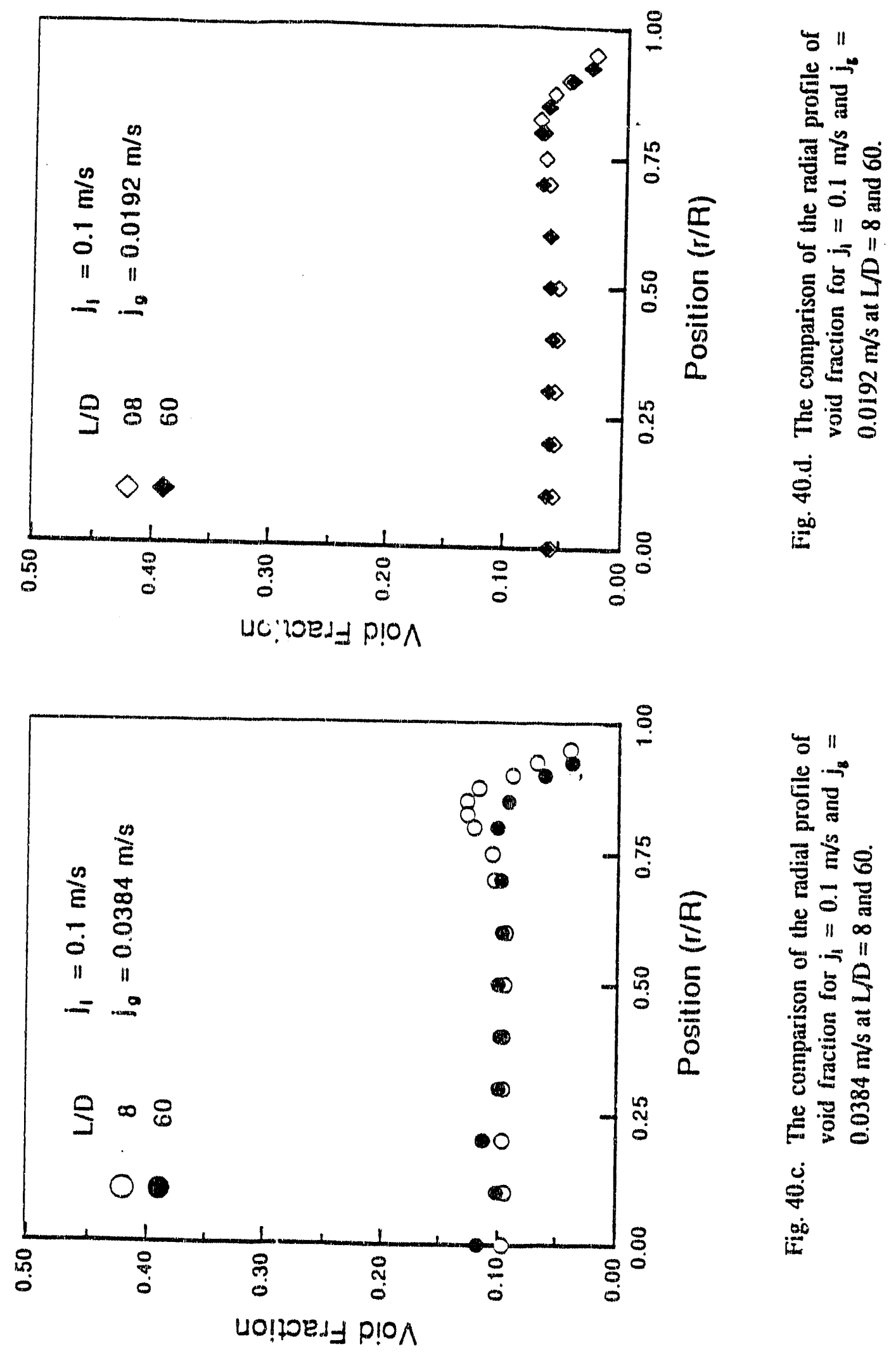

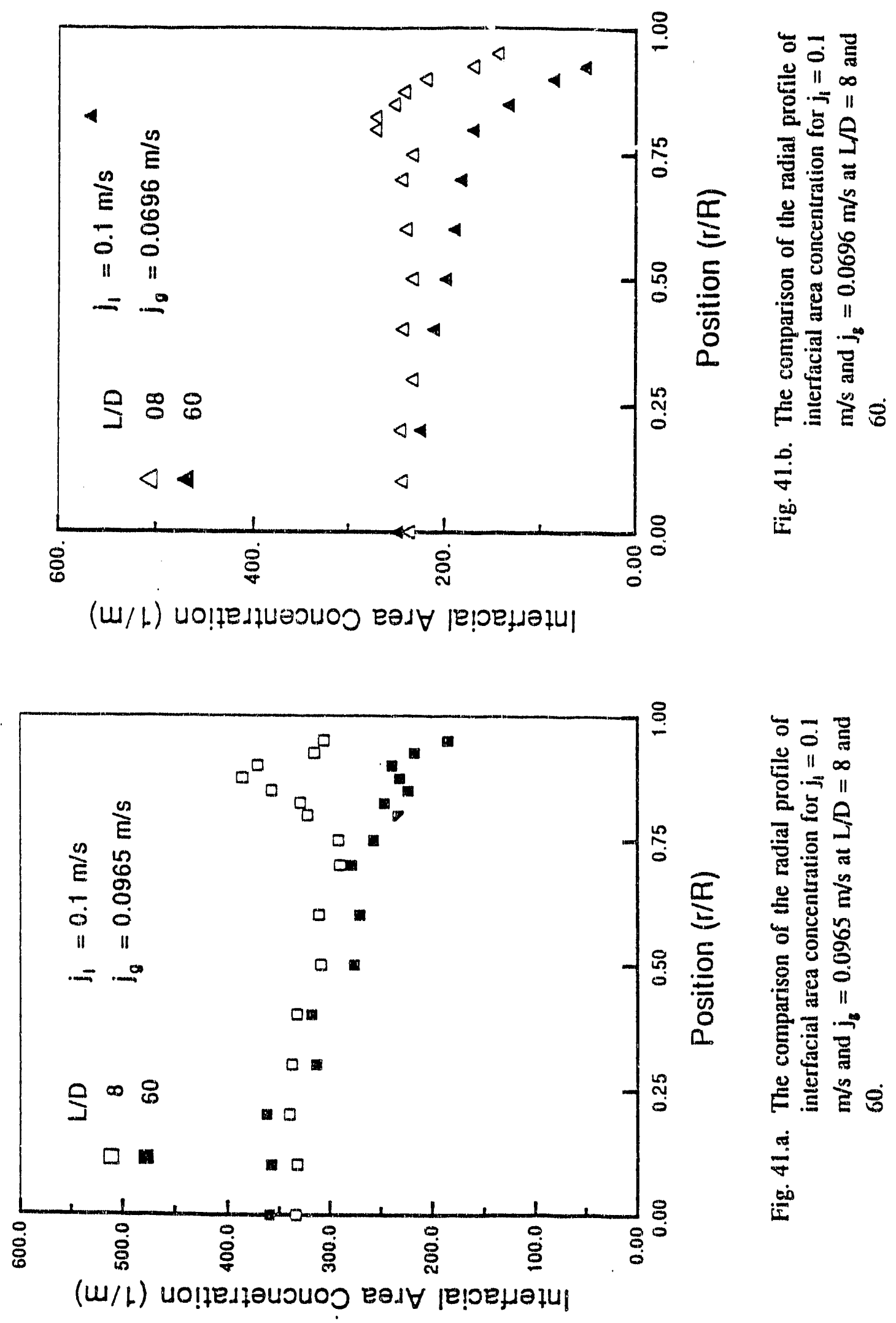

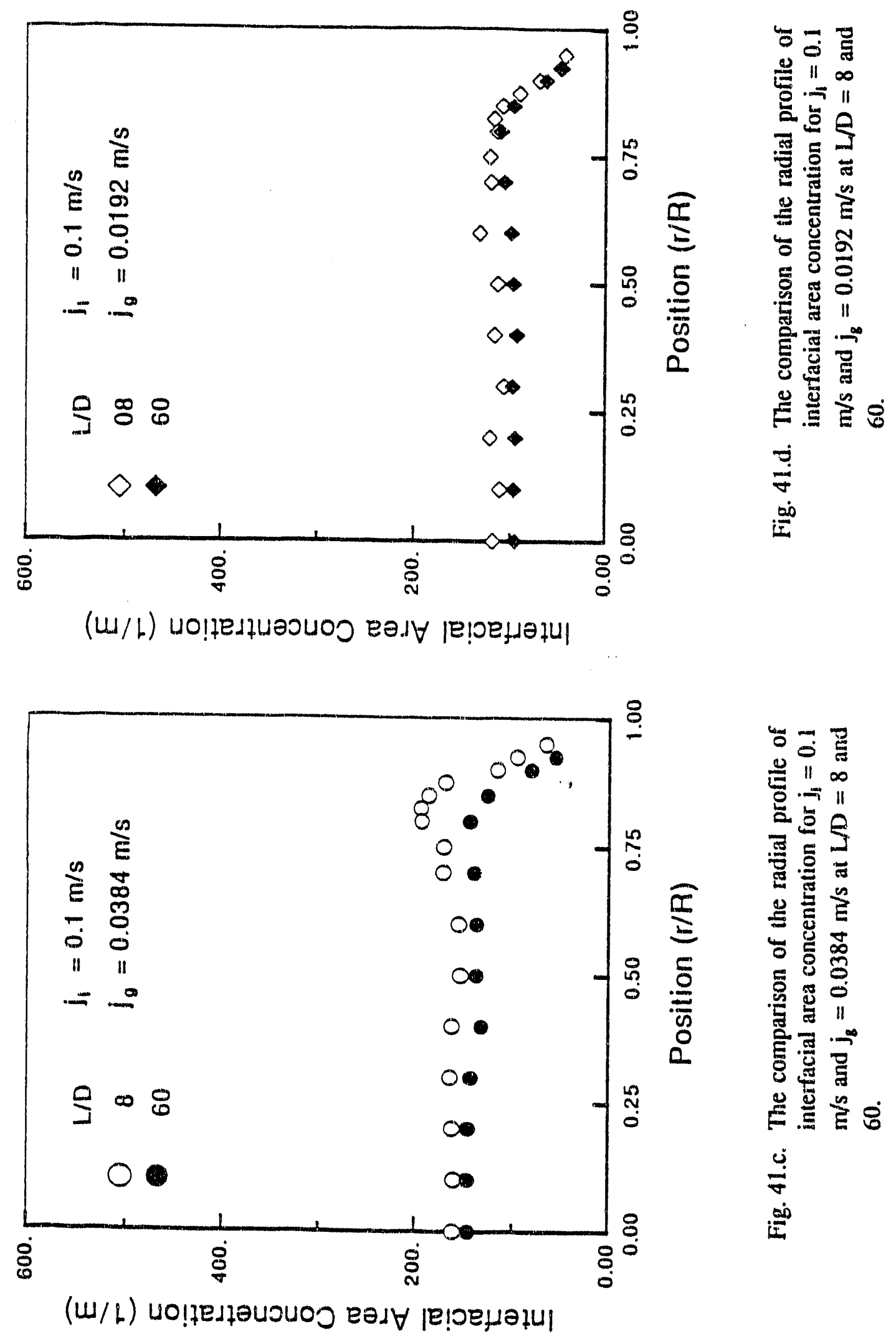

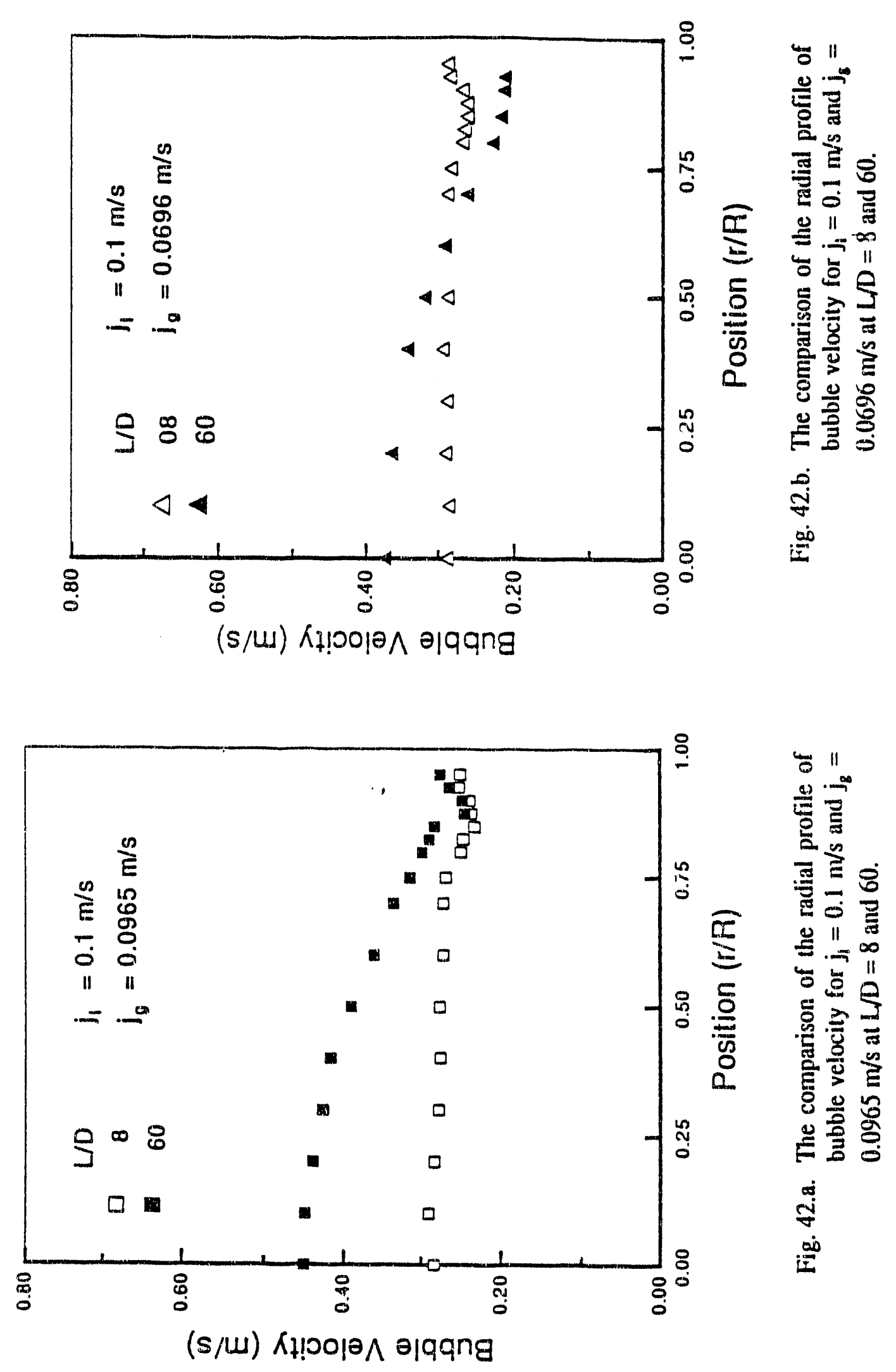

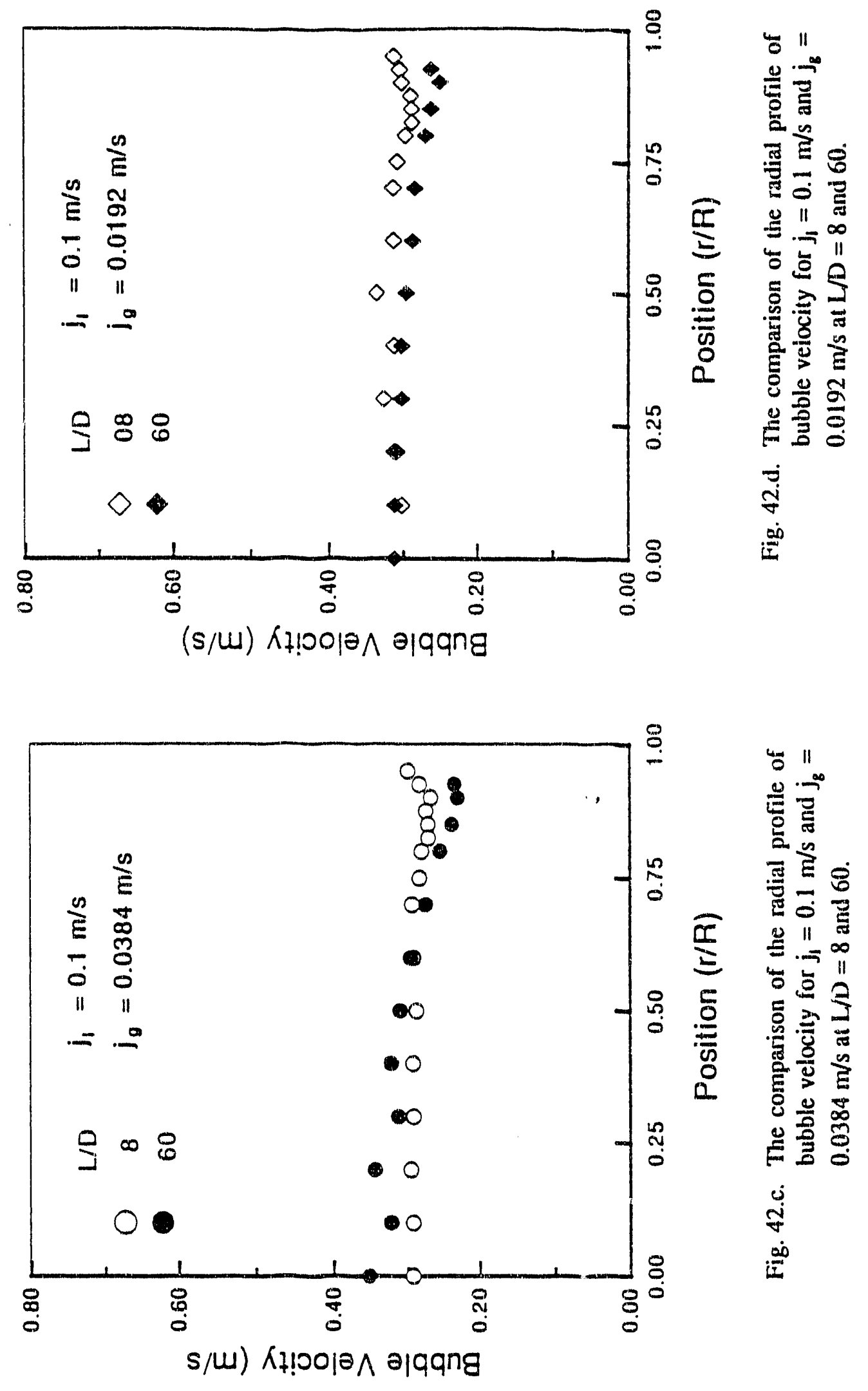

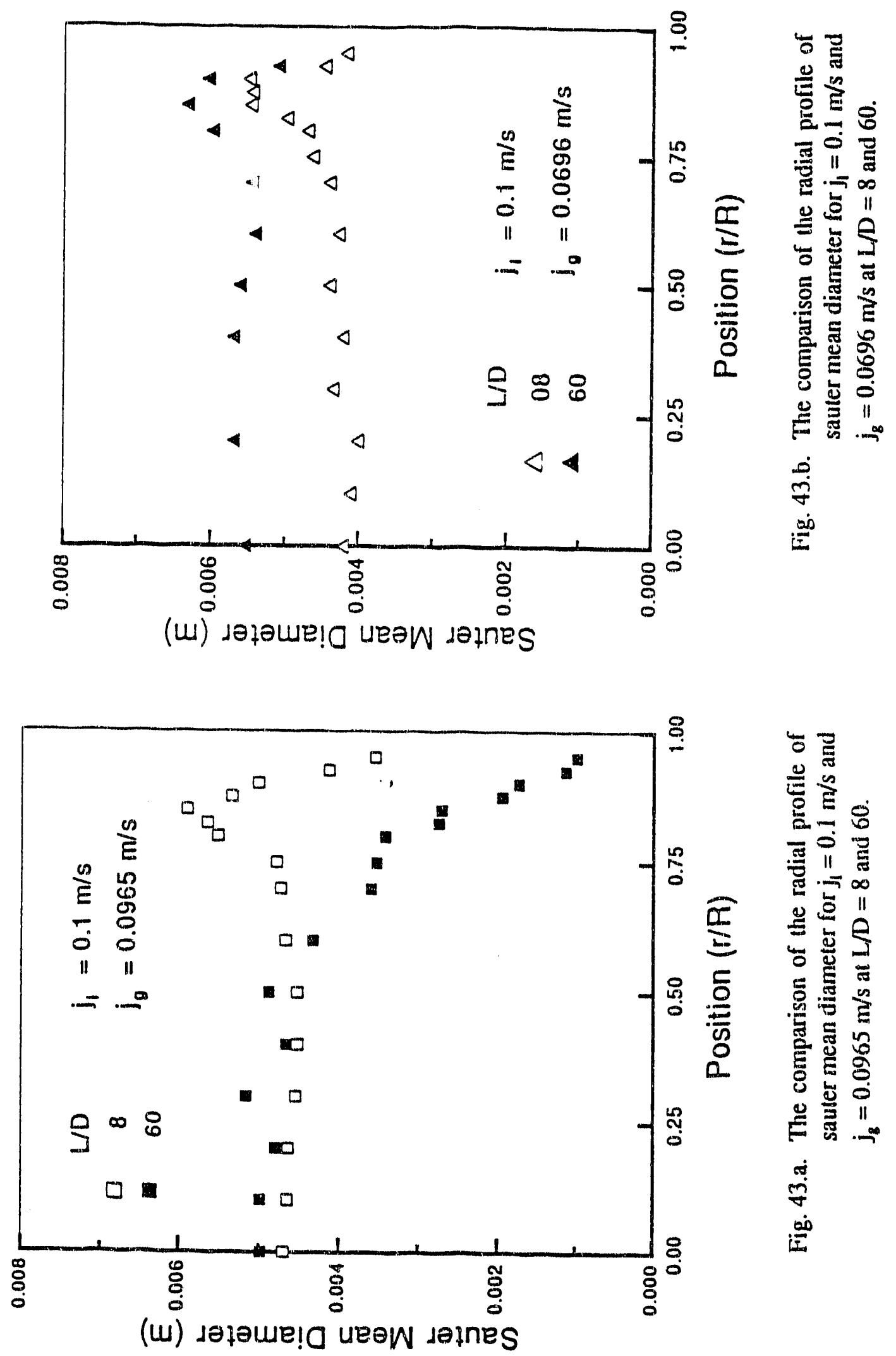

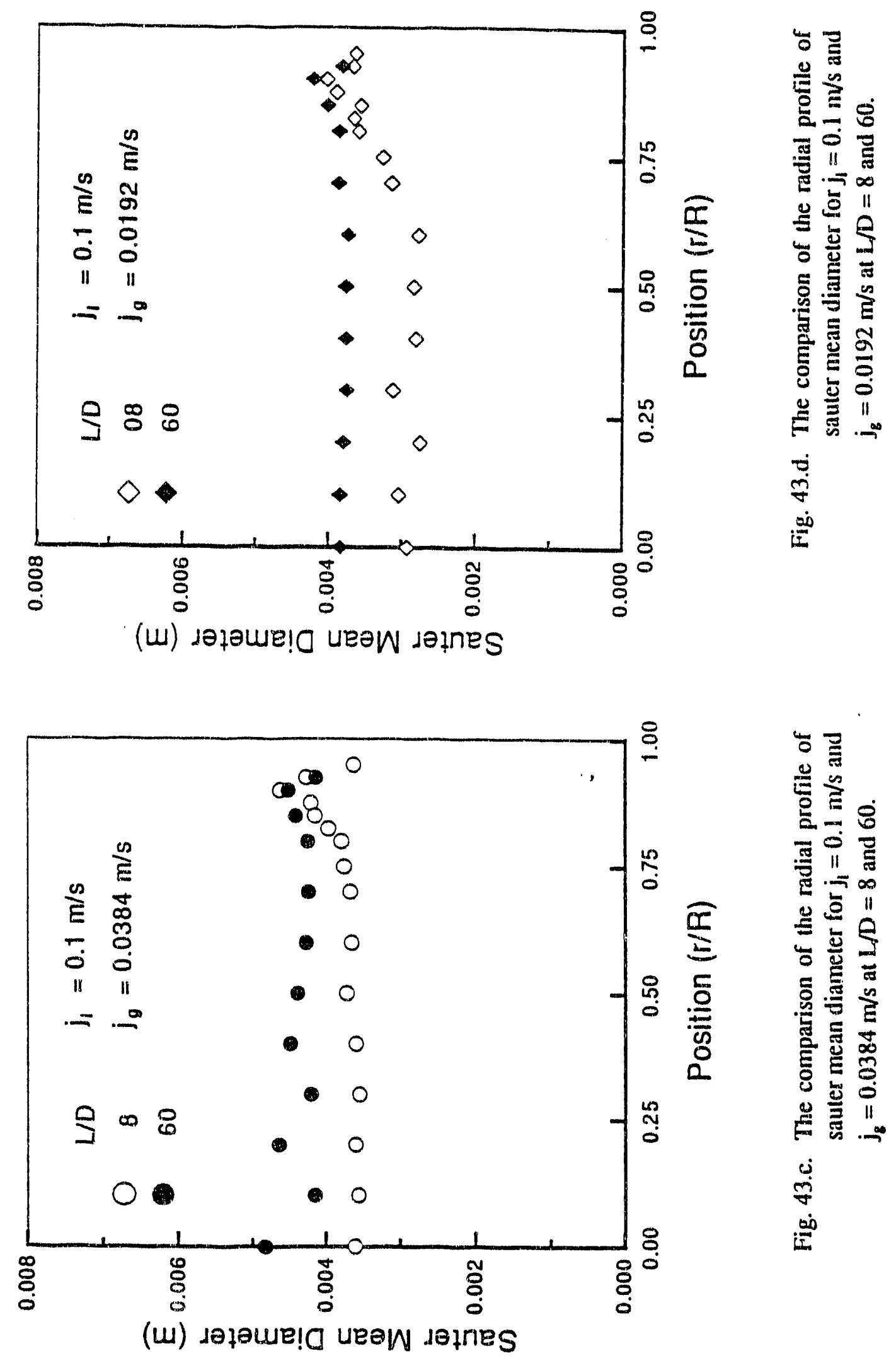

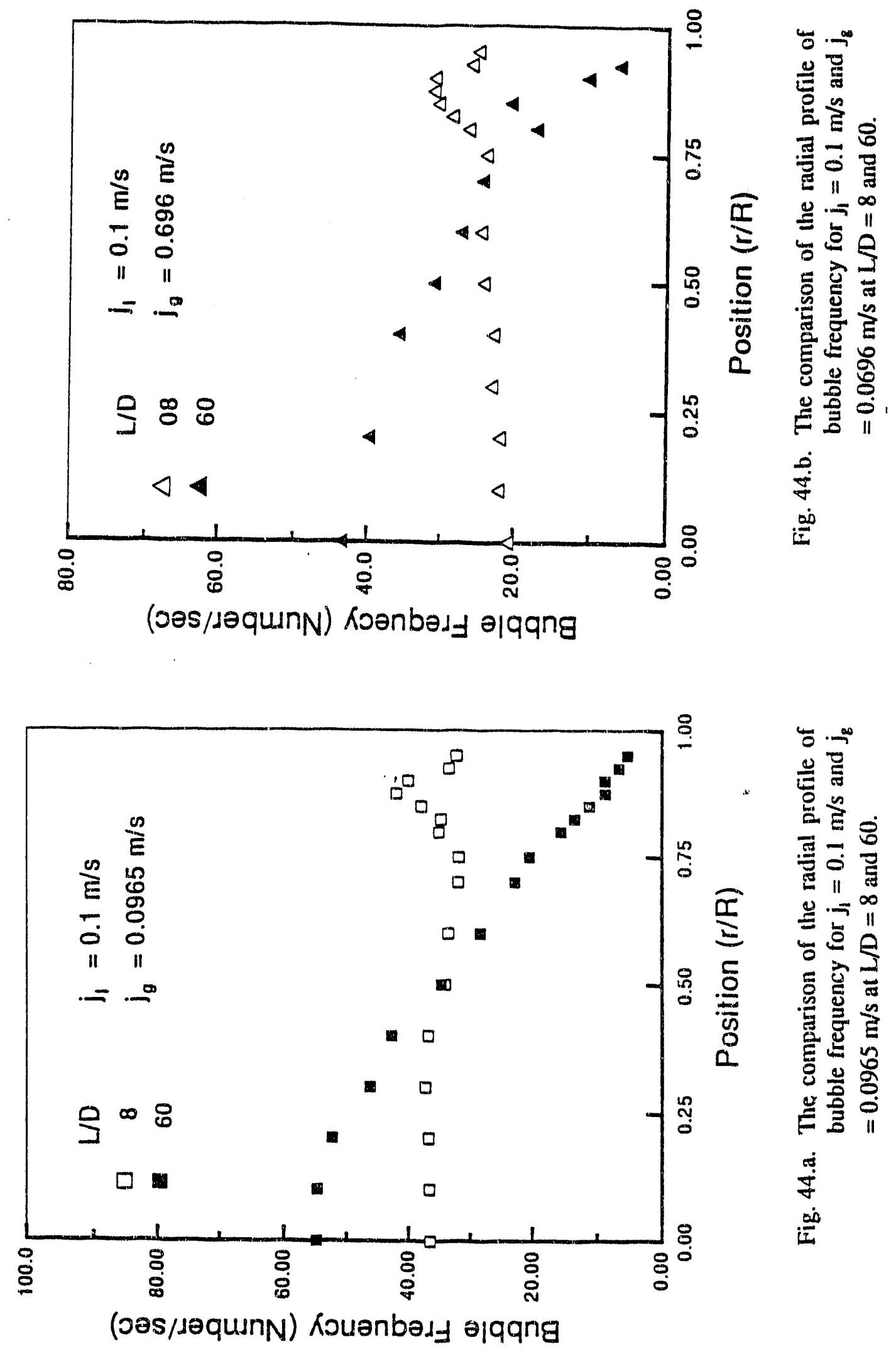

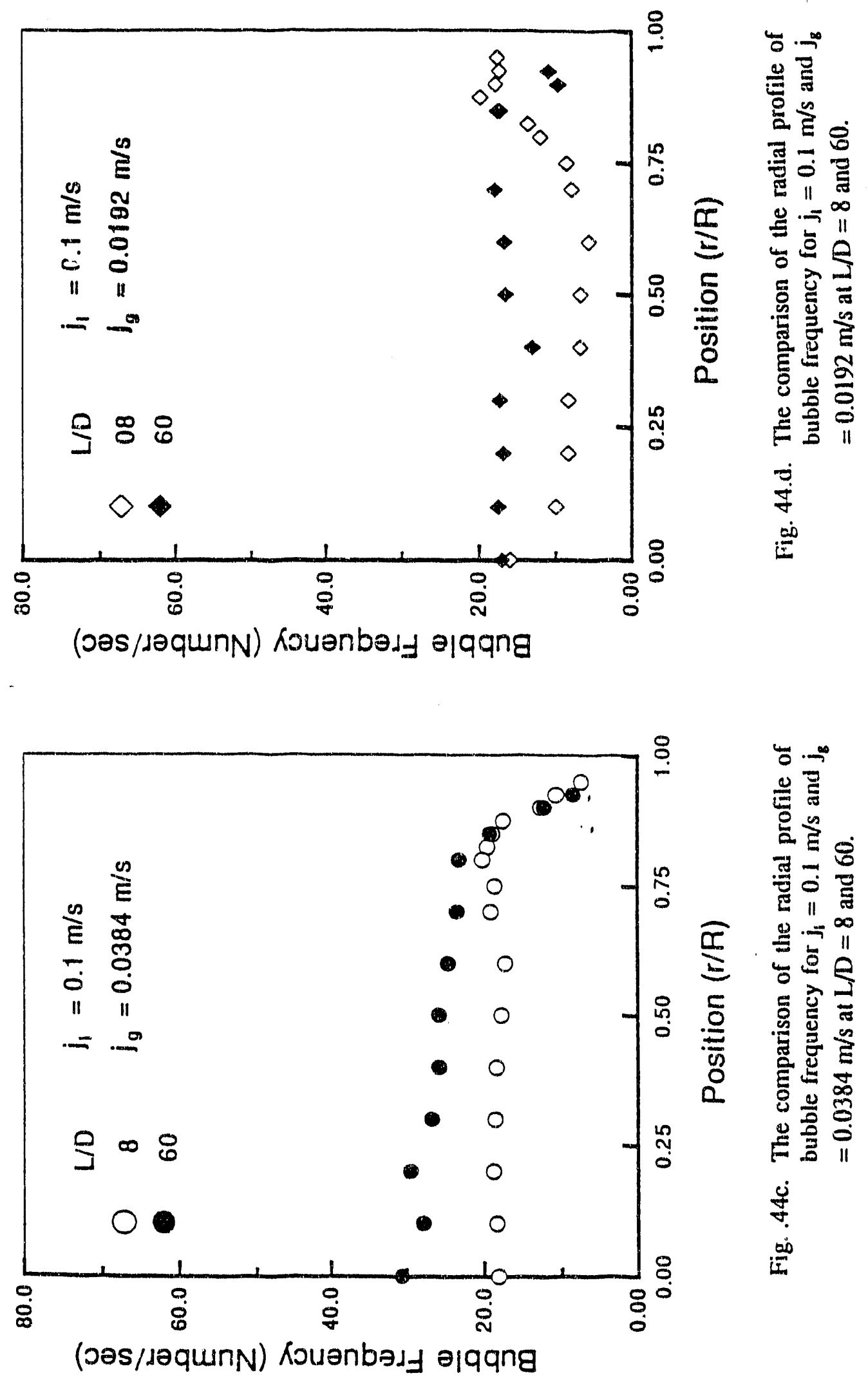


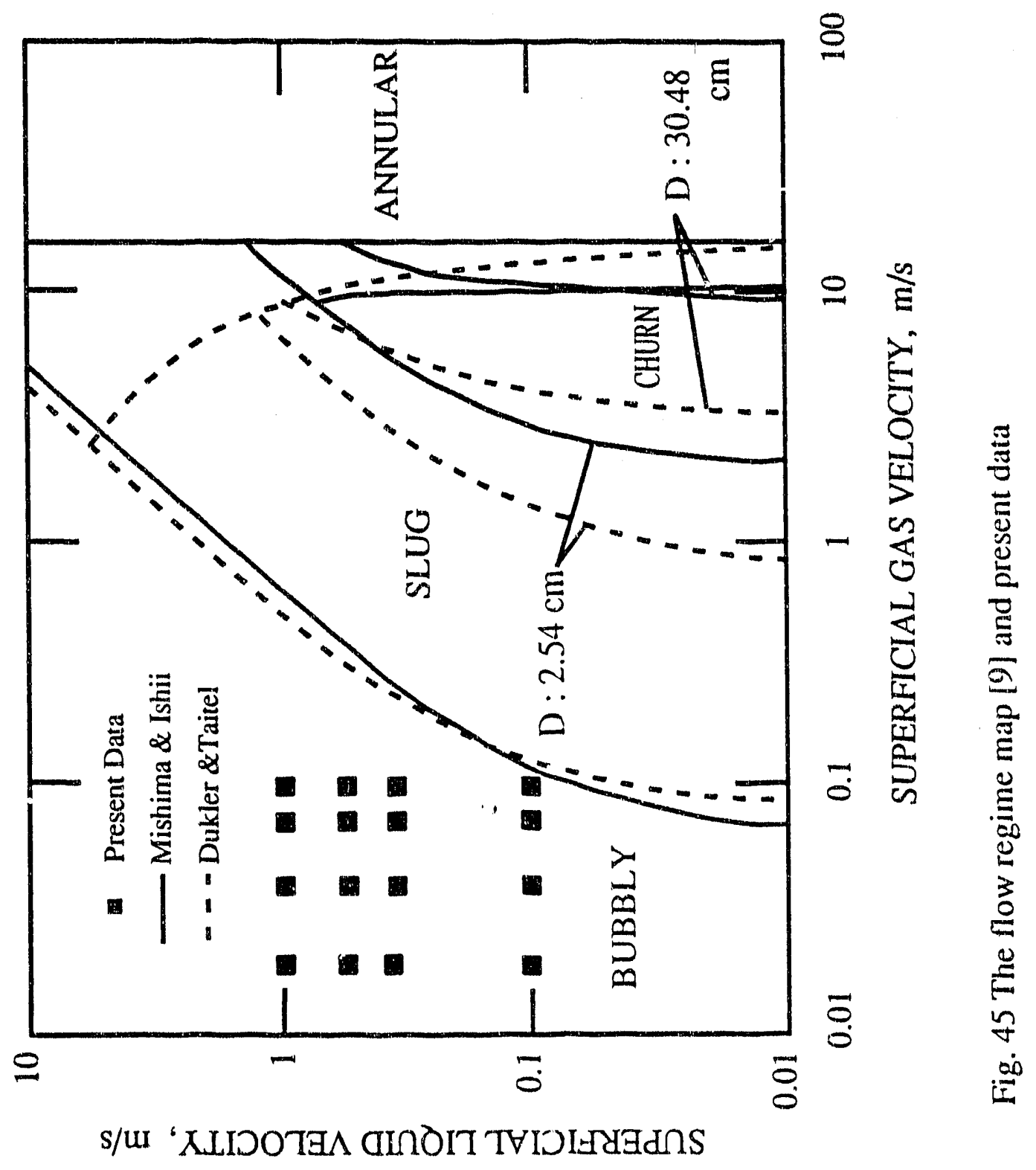




\subsection{The average characters}

\subsubsection{The Drift Flux Model}

Since the local liquid flow character is not measured and there is a lack of interfacial transport modelling, we do not have enough information to analyze the local phasic distribution phenomenor. The quasi one-dimensional model requires no interfacial information and smears out the detailed local phasic parameters by the average volumetric flux representation of the two-phase flow. The basic average one dimensional analysis is given by Zuber [28] anr' Ishii [1]. The drift flux relation:

$$
\frac{\left\langle j_{g}\right\rangle}{\langle\alpha\rangle}=\mathrm{C}_{o}\langle j\rangle+\left\langle\mathrm{V}_{g j}\right\rangle
$$

where the $\mathrm{j}_{g}, \mathrm{j}, \mathrm{C}_{o}$, and $\mathrm{V}_{\mathrm{g} j}$ are the superficial gas velocity, total volumetric flux, correlation coefficient, and drift velocity respectively. The angular blanket represents area average value on the transverse direction. The area average values can be calculated from the local radial profile by a numerical integration method. A simple rectangle rule was used to obtain the average value and the data points were the height of the rectangle stripes. The correlation coefficient is defined as:

$$
\mathrm{C}_{o}=\frac{\langle\alpha j\rangle}{\langle\alpha \lambda\langle j\rangle}=\frac{\int_{A} \alpha(\mathrm{r}) j(\mathrm{r}) d s}{\int_{A} \alpha(\mathrm{r}) d s \int_{A} j(\mathbf{r}) d s}
$$

where $\mathrm{C}_{o}$ accounts for the void fraction profile effect on the total volumetric flux. The drift flux plot is shown in Fig. 46. The average gas velocity is plotted against the total volumetric flux. The slope gives the value of $C_{o}$ and $y$-interception gives the gas phase drift velocity. The recommended value of $\mathrm{C}_{o}$ is 1.2 for bubbly flow. The best fit of the experimental data gives the slope $\mathrm{C}_{o}=0.7$ and the gas drift velocity is equal to $20 \mathrm{~cm} / \mathrm{s}$. It is expected because the correlation of the void distribution and the velocity profile in bubbly flow case should be smaller than one. At the entrance, the data points fit quite well with those values since all the void profiles show the wall peak. However, in the fully developed region, the lower volumetric flux cases, the data points converge toward $\mathrm{C}_{o}=1.2$ which is the value for the center core void peaking correlation. 

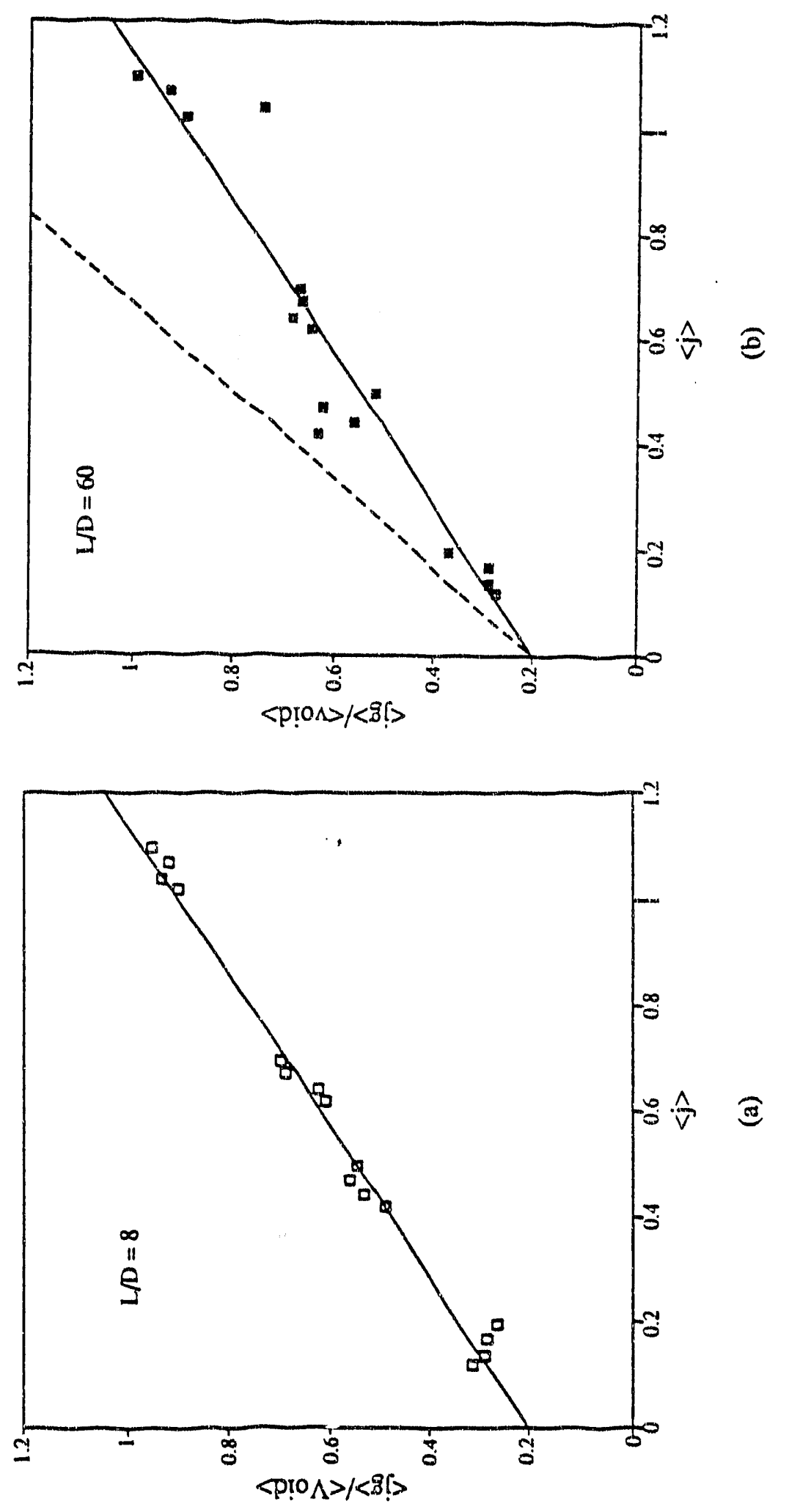

点 อ $\stackrel{\sim}{0} \stackrel{11}{i \mid g}$ 文 忥 仓ั 00 II II e 를 잉 త. 흠 픙 $\stackrel{x}{\Xi}$ 를 Е $\%$ 要 


\subsubsection{Comparison of Average Values}

The following two plots (Fig. 47.a and b) show the comparison of average void fraction and interfacial area concentration between the entrance and the fully developed zone. The average void fraction remains the same as they go downstream. For the two cases of highest void fraction, the average void fractions gets smaller than at the entrance region. For $j_{l}=$ $0.1 \mathrm{~m} / \mathrm{s}$ with $j_{8}=0.0956$ and $0.0696 \mathrm{~m} / \mathrm{s}$ the highest average void fraction exist and bubbly slugs are found in the fully develcped region. From the local velocity plots (see Fig. 42.a and $42 . b$ ), the local bubble velocities increase in the fully developed region, and these constitute a higher local slip ratio. Thus the void fraction is getting smaller in the fully developed region. Comparing the Interfacial area concentrations between the two regions, they are very much the same. But at the high value of interfacial area concentration, the data points scanter around the $45^{\circ}$ line. For those cases, the error arises from both the velocity and void fraction measurements in high bubble concentration.

The next six figures (see Fig. 48 to 50) are the average interfacial area concentration versus the average void fraction. The near wall region is for $1 \geq r / R \geq 0.775$ and the core region is for $0.775 \geq r / R \geq 0$. Most of the data points scatter around a straight line. In the cases of high void fraction, the interfacial area concentration takes on a smaller value which indicates a larger average bubble ize with less interfacial area per unit volume. The reciprocal of the slope is proportionai to the sauter mean diameter. Using the slope of the best fitted line, the average sauter mean diameter which is calculated from the slopes and they are:
i). L/D $=8$, near wall region
$D_{\text {itm }}=4.00 \mathrm{~mm}$
ii). $L / D=8$, core region
$D_{\mathrm{sm}}=3.2 .5 \mathrm{~mm}$
iii). $L / D=8$, area average
$D_{\mathrm{sm}}=3.50 \mathrm{~mm}$
iv). I/ $D=60$, near wall average
$D_{\text {sm }}=4.00 \mathrm{~mm}$
v). $L / D=60$, core region
$D_{\mathrm{smm}}=3.50 \mathrm{~mm}$
vi). $L / D=60$, area average
$D_{\mathrm{sm}}=3.65 \mathrm{~mm}$

The average sauter mean diameter at the near wall region is larger than the core region. From the entrance to the developed region, the overall average diameter is also getting larger which indicates that there is the same coalescence as the two-phase mixture flows downstream. 

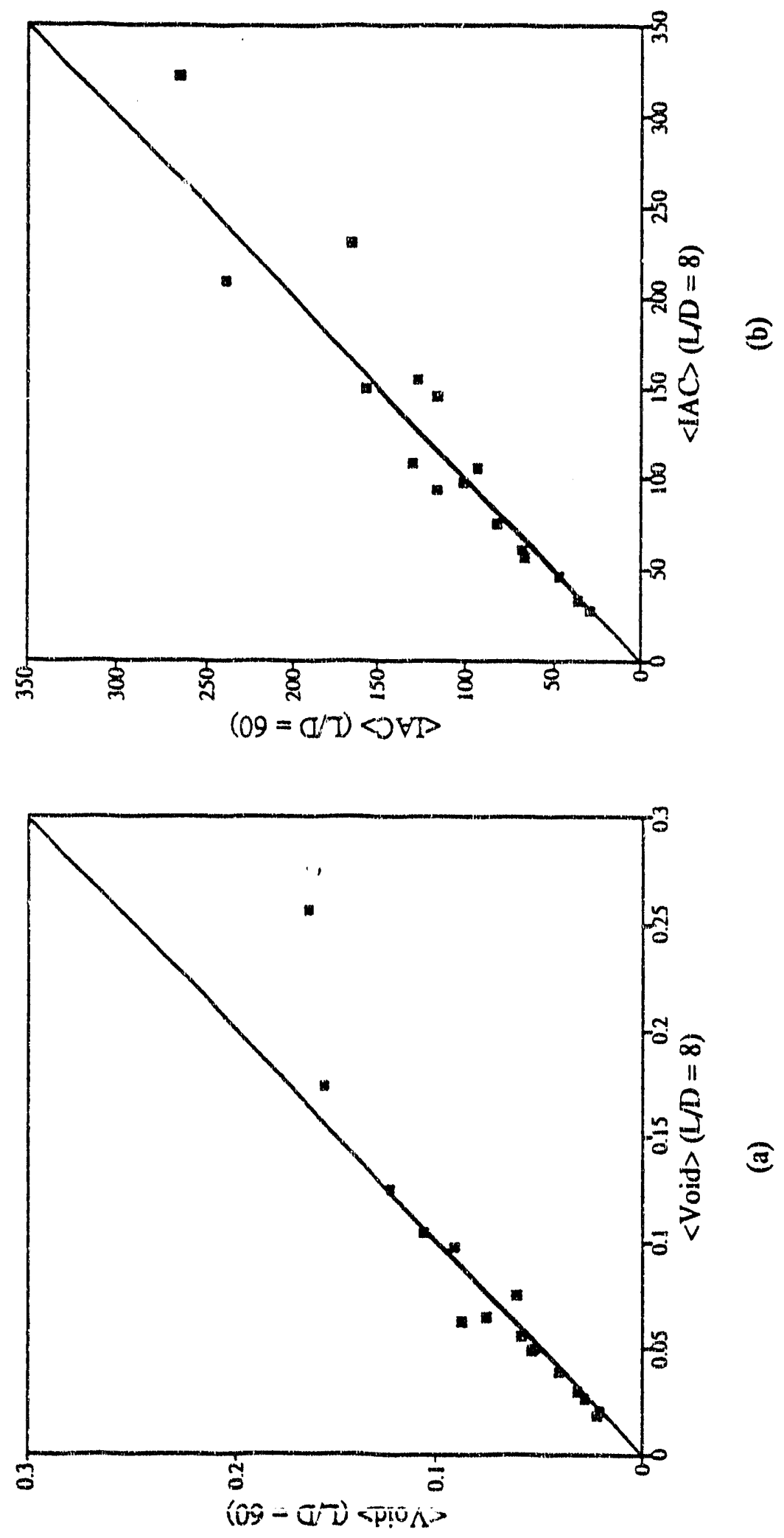

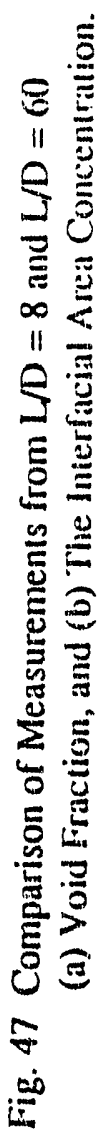




\subsubsection{Comparison of Average Values}

The following two plots (Fig. 47.a and o) show the comparison of average void fraction and interfacial area concentration between the entrance and the fully developed zone. The average void fraction remains the same as they go downstream. For the two cases of highest void fraction, the average void fractions gets smaller than at the entrance region. For $j_{l}=$ $0.1 \mathrm{~m} / \mathrm{s}$ with $j_{g}=0.0956$ and $0.0696 \mathrm{~m} / \mathrm{s}$ the highest average void fraction exist and bubbly slugs are found in the fully developed region. From the local velocity plots (see Fig. 42.a and $42 . b$ ), the local bubble velocities increase in the fully developed region, and these constitute a higher local slip ratio. Thus the void fraction is getting smaller in the fully developed region. Comparing the Interfacial area concentrations between the two regions, they are very much the same. But at the high value of interfacial area concentration, the data points scatter around the $45^{\circ}$ line. For those cases, the error arises from both the velocity and void fraction measurements in high bubble concentration.

The next six figures (see Fig. 48 to 50 ) are the average interfacial area concentration versus the average void fraction. The near wall region is for $1 \geq r / R \geq 0.775$ and the core region is for $0.775 \geq r / R \geq 0$. Most of the data points scatter around a straight line. In the cases of high void fraction, the interfacial area concentration takes on a smaller value which indicates a larger average bubble size with less interfacial area per unit volume. The reciprocal of the slope is proportional to the sauter mean diameter. Using the slope of the best fitted line, the average sauter mean diameter which is calculated from the slopes and they are:

i). $\quad \mathrm{L} / \mathrm{D}=8$, near wall region

$\mathrm{D}_{\mathrm{sm}}=4.00 \mathrm{~mm}$

ii). $\quad L / D=8$, core region

$D_{s m}=3.25 \mathrm{~mm}$

iii). $\quad L / D=8$, area average

$\mathrm{D}_{\mathrm{sm}}=3.50 \mathrm{~mm}$

iv). $\quad L / D=60$, near wall average

$\mathrm{D}_{\mathrm{sm}}=4.00 \mathrm{~mm}$

v). $L / D=60$, core region

$\mathrm{D}_{\mathrm{sm}}=3.50 \mathrm{~mm}$

vi).

$L / D=60$, area average

$\mathrm{D}_{\mathrm{sm}}=3.65 \mathrm{~mm}$

The average sauter mean diameter at the near wall region is larger than the core region. From the entrance to the developed region, the overall average diameter is also getting larger which indicates that there is the same coalescence as the two-phase mixture flows downstream. 

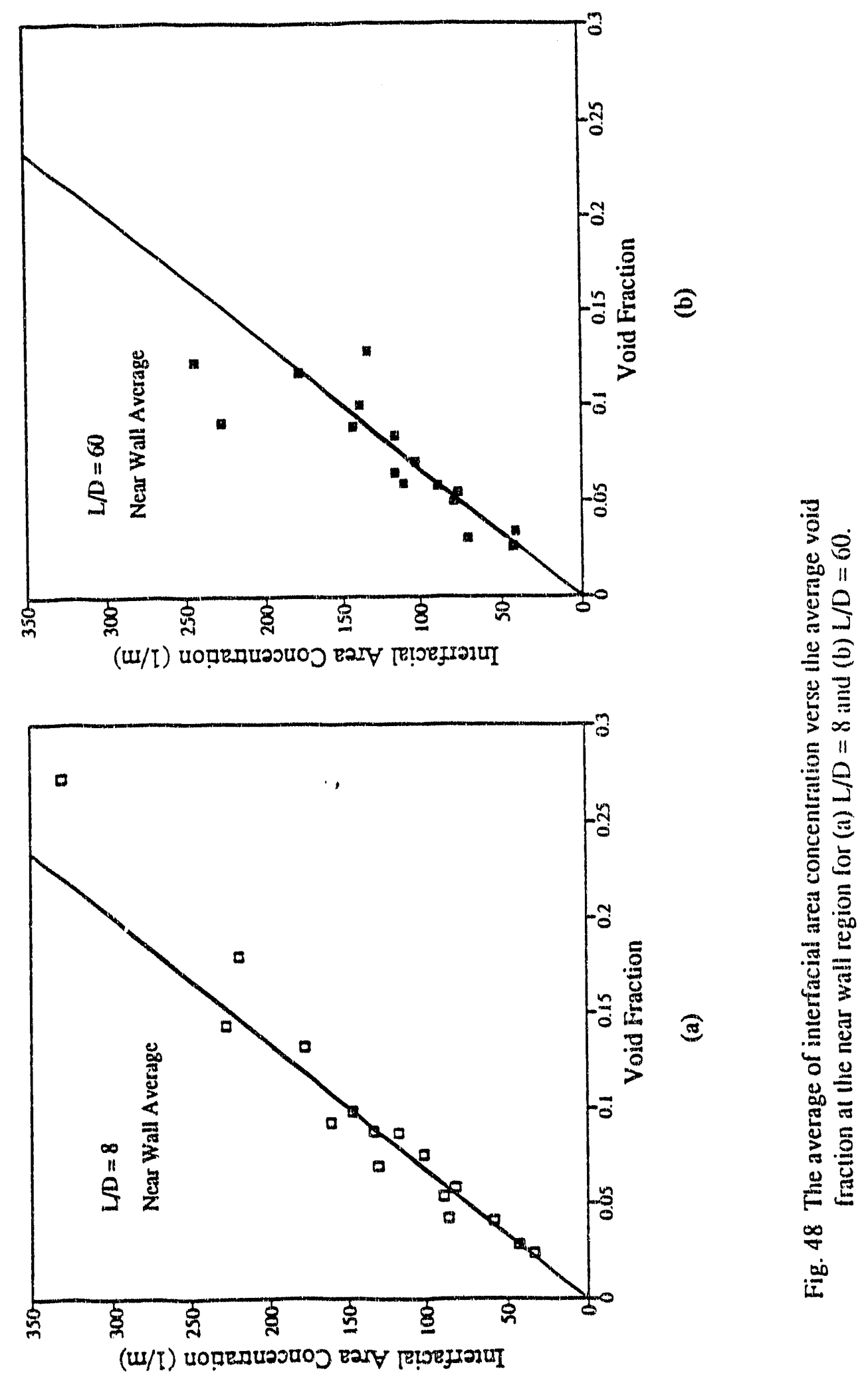

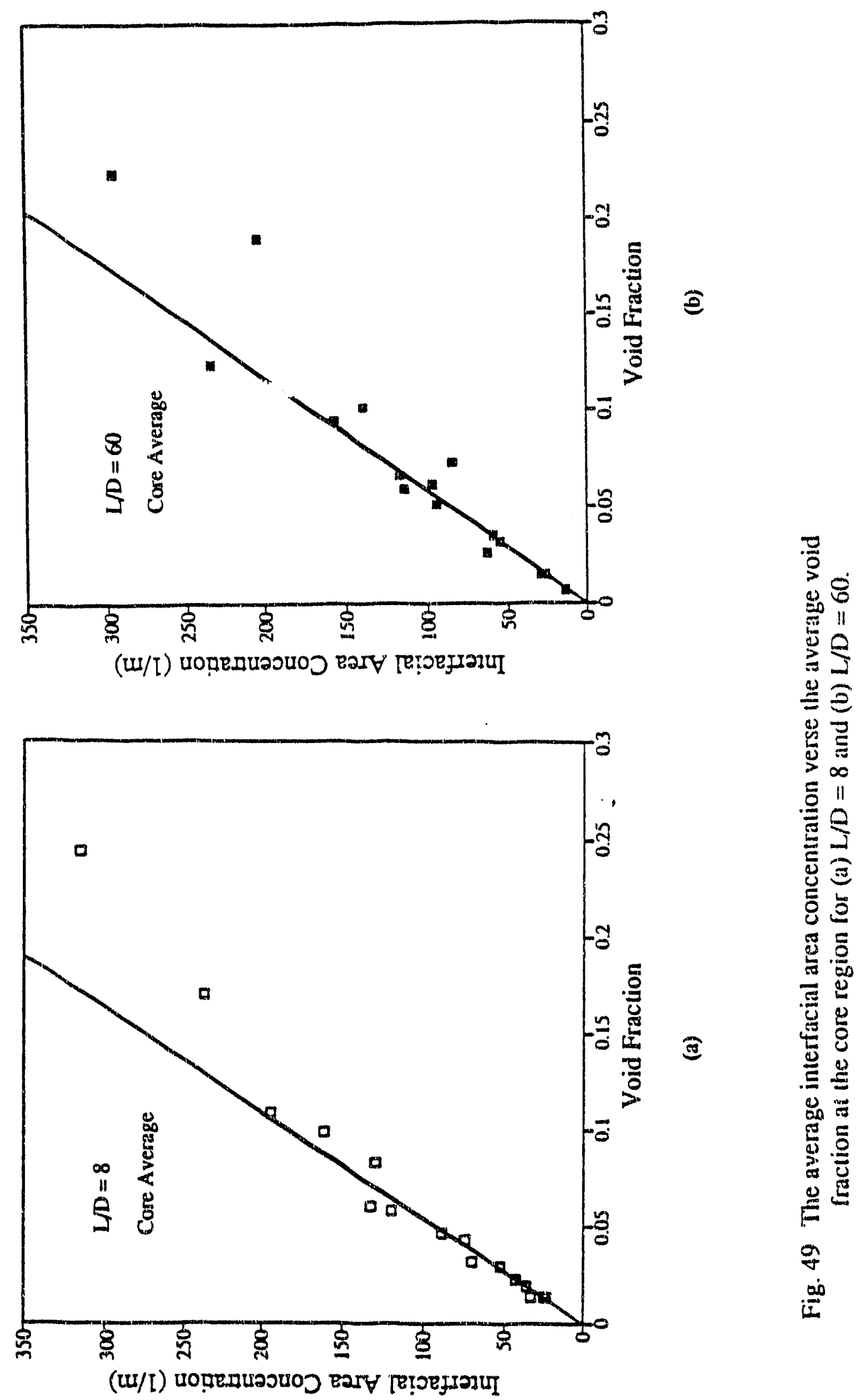

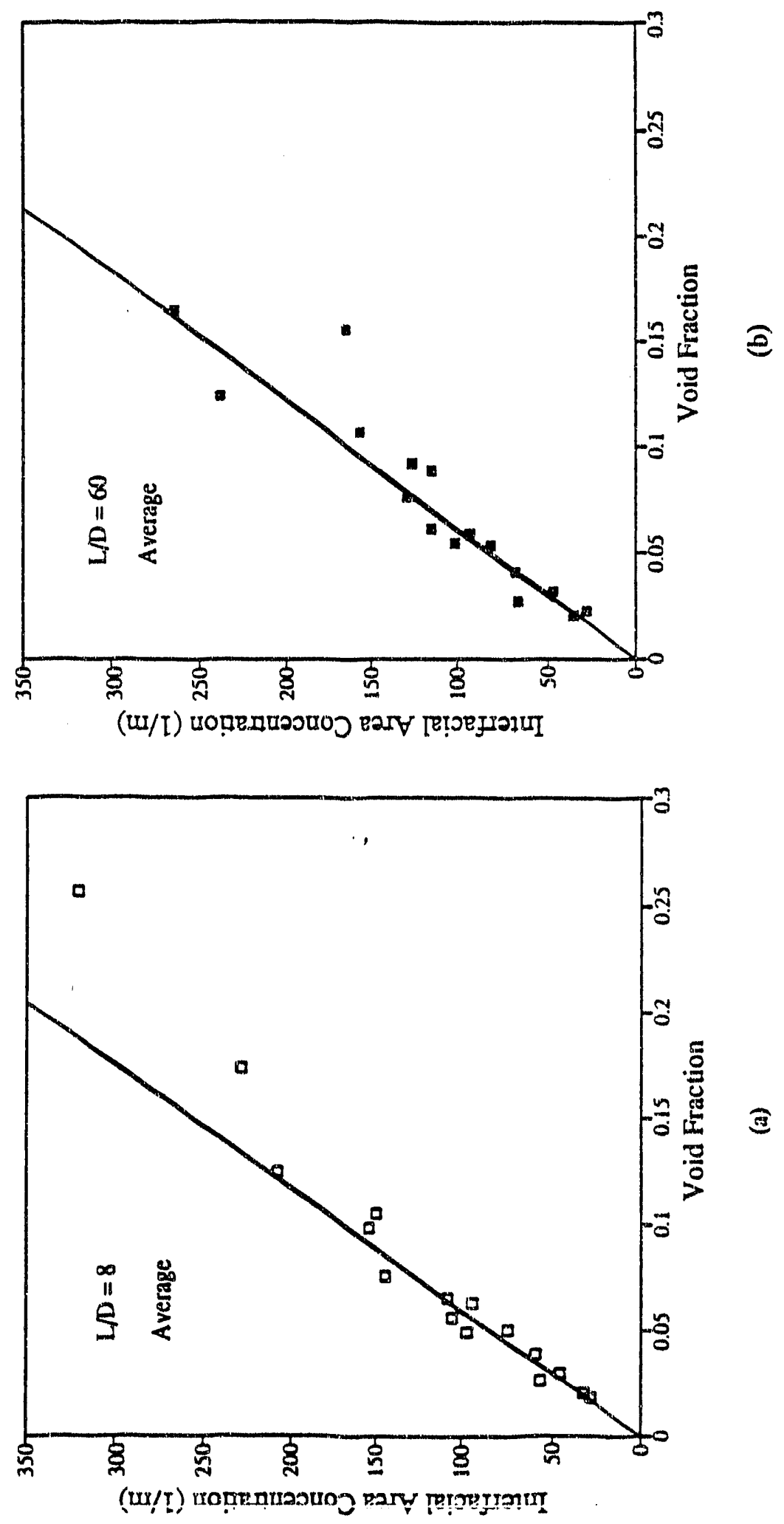

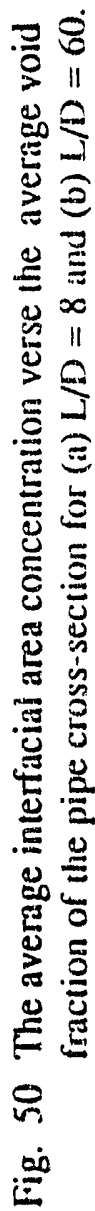



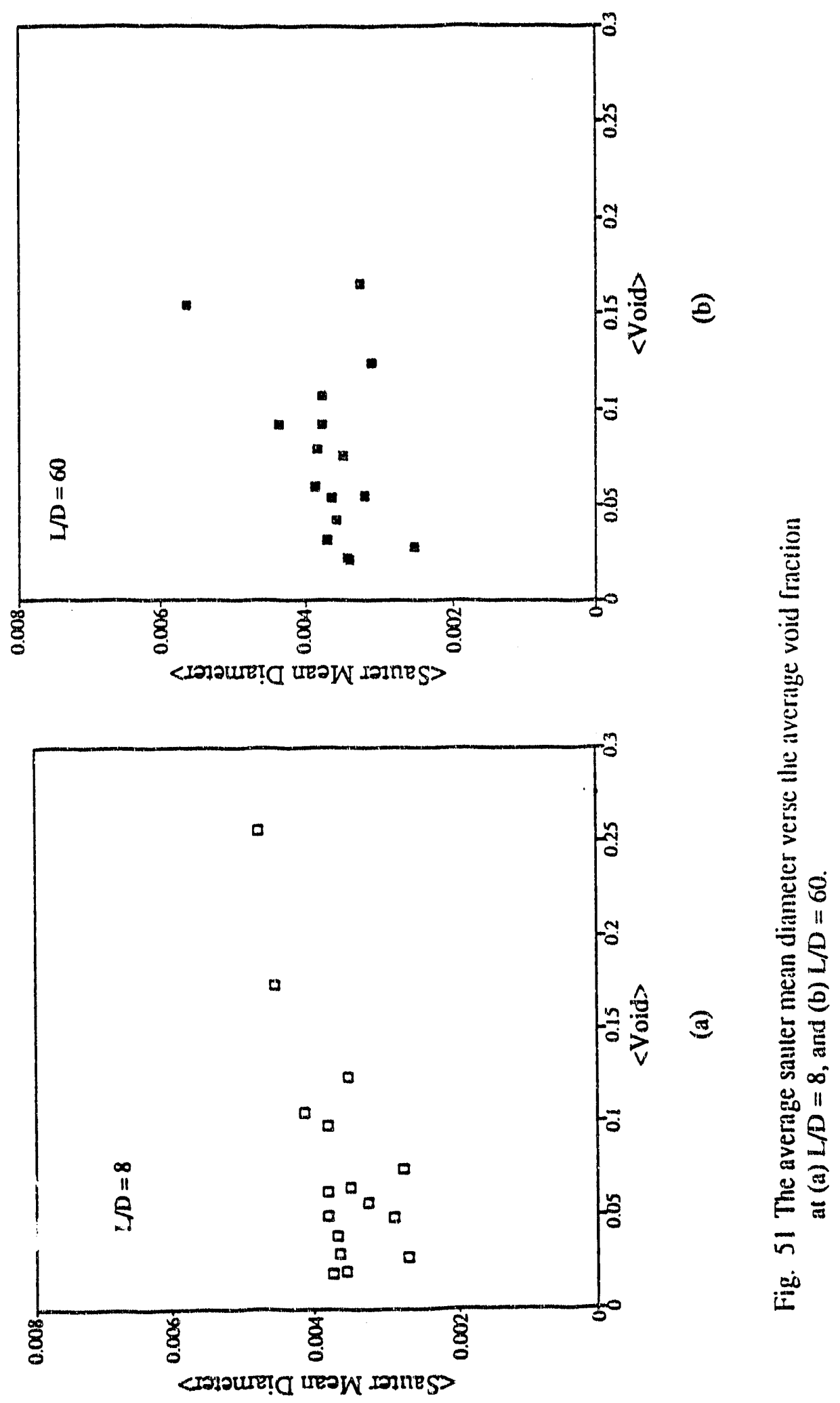

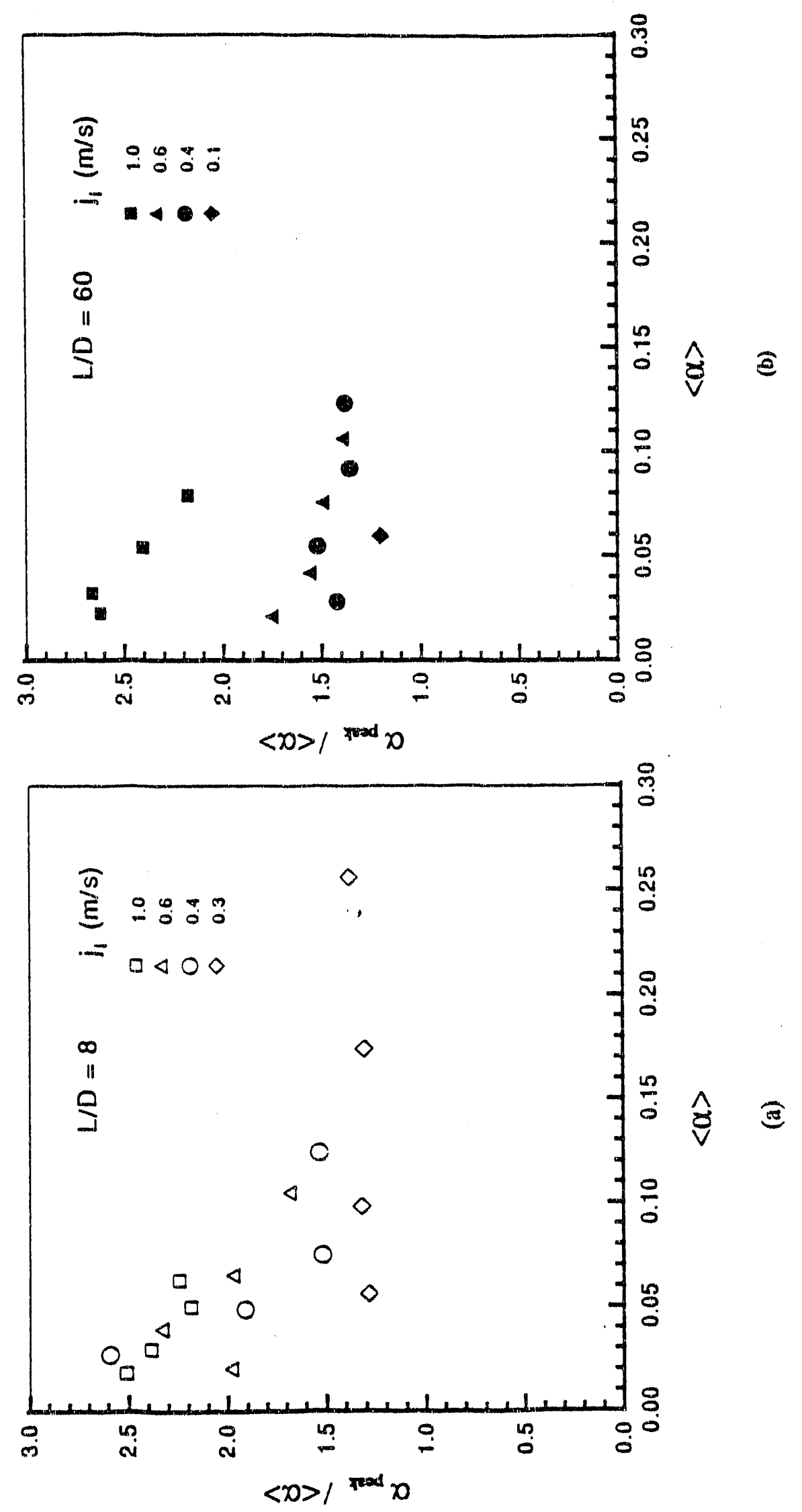
Fig. 51 contains the plots of the area averaged local sauter mean diameter versus the average void fraction. The average diameter is roughly $3.8 \mathrm{~mm}$ and increases a little with void fraction, but it does not depend on the liquid velocity. The lowest mean diameters occur at $\mathrm{j}_{l}=0.4 \mathrm{~m} / \mathrm{s}$, there may be measurement error of the velocity measurements since there is no apparent reason for the smaller bubble size. In the case of $j_{l}=0.1 \mathrm{~m} / \mathrm{s}$, the sauter mean diameter increases quite drastically to $6 \mathrm{~mm}$, and the reason for this was mentioned in the previous discussion.

The last two figures, Fig 52a and 52b, the void peak ratio versus the average void fraction are plotted and data points are grouped under the same $\mathrm{j}_{l}$. At the entrance region, the peak strongly depends on the average void fraction regardless of the volumetric flow rate. The peak decreases as the void fraction increases. The ratio runs from 2.5 to 1.5 in all cases. The higher of the average void fraction, the lesser the percentage of the bubbles concentrate at the wall region. For the superficial liquid velocity $j_{l}=0.1 \mathrm{~m} / \mathrm{s}$, the average to peak ratio is roughly 1.5 regardless the void fraction. In the fully developed region, the void peak diminishes in the cases of $j_{l}=0.4$ and $0.6 \mathrm{~m} / \mathrm{s}$ and it becomes less dependent on void fraction. Only the $j_{l}=1.0 \mathrm{~m} / \mathrm{s}$ case shows that the void peak at the developed region is pushed to a higher value then at the entrance region. The phasic distribution mechanisms are probably different at $L / D=3$ and $L / D=60$. At the entrance region, bubbles may be accumulated near the wall due to the flow is converging from a larger area section. For the developed region, the liquid flow character may be responsible for either keeping or dispersing the bubbles at the wall. The higher superficial liquid velocity implies the higher turbulent intensity, but the local turbulent structure is hard to predict because it is modified by the dispersed phase. Further study on the two-phase flow turbulent structure is needed in order to understand the phase distribution mechanism in dispersed flow regime. 


\section{Conclusion}

We have improved the design and miniaturization of the double sensor probe. The signal processing program is also retined with a new algorithm of interface detection. A trace of the probe signal shows there is a rather long rise time due to the dry out process and the long time constant. This kind of delay poses a limitation on the fast detection for a direct current biasing probe. More studies are required to improve the probe biasing circuit for measurements in high volumetric flow rate. On the other hand, in the low liquid velocity cases, we find the an accurate measurement is harder to get and the probe signal is quite fuzzy. If the interface is slow and distorted, the sensor cannot pinch cleanly through interface instantaneously. Since we haven't studied the interface sensor piercing mechanism, it remains a speculation only. Further study on the velocity effect on the interface piercing can help to improve the accuracy of the probe measurements. The overall performance of the double-sensor probe is satisfactory and it gives a fairly accurate local measurement.

The double-sensor probe interfacial area concentration measurement method is based on the algorithm developed by Kataoka et al. It has not been verified by any other independent method at this point. Nevertheless, from the measurements of the bubble frequency, chord length and the photographic studies done by previous and present experiments, it is shown that the interfacial area concentration assumption is consistent with the results. For instance, as a higher number of the bubbles concentrating near the wall give a higher local void fraction. The available surface area in the local region also increases due to the higher bubble concentration. A good interfacial area measurement should reflect such phenomenon. As a matter of fact, all of the radial interfacial area concentration profiles show agreement with the local void fraction measurement. The sauter mean diameter is cornputed by Eq. (43) and it is reciprocally proportional to the interfacial area concentration. The value of the sauter mean diameter reflects the accuracy of the interfacial area concentration. The measured sauter mean diameter is roughly $3.8 \mathrm{~mm}$, which also agrees with previous experiments. It shows that the algorithm gives a satisfactory result on the interface geometry measurement. However, a direct proof is always desirable but it requires a miniaturized three double-sensor probe to measure all three components of the interface velocity vector.

The average sauter mean diameter increases with the void fraction, and the liquid velocity has no or little effect on bubble sizes. Locally, the sauter mean diameter seems to be getting larger at the pipe wall region. This may be due to the bubble orientation rather 
than the actual increase of the bubble size. For $\mathrm{j}_{l}=0.1 \mathrm{~m} / \mathrm{s}$, the bubble mean diameter does increase quite drastically for higher gas injection rates. The high void diameter increases the probability of bubble coalescence and agglomeration to form a bubbly slug.

At the entrance, the near wall void peak is found in all cases of flows. The void peak is well established at the entrance region and it diminishes in the fully developed region if the liquid flows at a moderate rate. The void profile may be formed by bubbles accumulating at the wall while they are passing through the convergent section. Since our bubble generation is not intended to get an even bubble distribution at the entrance, we cannot give a good estimation on what exactly happened. However, as the flow develops in the test section, the liquid flow is more responsible for keeping the bubble close to the wall. In the case of $j_{l}=1 \mathrm{~m} / \mathrm{s}$, the void peaks become higher in fully developed region. Since higher liquid velocity means higher turbulent intensity, the liquid phase eddies may be responsible for the high bubble concentration near the pipe wall. We should study more in the high velocity regimes and measuring the liquid flow characters in order to clarify this point. However, the maximum liquid velocity that the purnp can handle is $1 \mathrm{~m} / \mathrm{s}$ in a 2 " id section. The higher flow rate can be obtained if an 1" id section is used. Then we can see more clearly whether the liquid eddy is part of the cause of the bubbles concentrating near the wall in bubbly flow.

On the other hand, in the case of $j_{l}=0.1 \mathrm{~m} / \mathrm{s}$, the local void fraction profile changes fro: a typical bubbly flow to slug flow character as the flow is developing. The bigger sauter mean diameter implies that the bubbles do coalesce in the developing region, but they don't merge into a single slug bubble. As a matter of fact, the bubbles agglomerate together to form a bubbly slug and the local bubble velocity increases because, in a closely packed slug, bubbles are entrained to the wake of the previous bubbles. The same phenomenon is observed in the churn turbulent flow.

Further studies on the average character from the local measurement have confirmed the local probe results are accurate. The success of the double probe measurement is relied on the statistically well behaved interface motions such as in bubbly flow. If the statistical character of the interface is arbitrary, we need to measure all three components of interfacial velocity. In more general local measurements, a three-double-sensor probe is needed for studying the more complicated phasic behavior. 


\section{Acknowledgments}

This work was performed under the auspices of the U.S. Department of Energy, Office of Basic Energy Science. The authors would like to express their sincere appreciation for the encouragement, support and technical comments on this program from Dr. O.P. Manly of DOE/BES. Special thanks to Ms. T. Wilmarth and Mr. C. Eberle for reading through the manuscript and giving the valuable comments. At last, but not the least, we would like to express the gratitude to our colleagues in the Thermal Hydraulic and Reactor Safety Laboratory. 


\section{References}

1. Ishii, M., "One-Dimensional Drift-Flux Model and Constitutive Equations for Relative Motion Between Phases in Various Two-Phase Flow Regimes," Argonne National Laboratory Report, ANL-77-47 (1977).

2. Ishii, M., "Thermo-Fluid Dynamic Theory of Two-Phase Flow", Eyrolles, Paris, or Scientific and Medical Publication of France, N.Y. (1975).

3. Ishii, M., T. C. Chawla, and N. Zuber, "Constitutive Equation for Vapor Drift Velocity in Two-Phase Annular Flow," AIChE J., 22,283 (1976).

4. Ishii, M., and Mishima K., "Study of Two-Fluid Model and Interfacial Area," Argonne National Laboratory Report, ANL-80-111, NUREG.CR-1873 (1980).

5. Kataoka I., Ishii M. and A. Serizawa," Local Formulation of Interfacial Area Concentration in Two-Phase Flow." Argonne National Laboratory Report,ANL-8468 NUREG/CR -4029 (1984).

6. Kataoka I., Ishii M. and A. Serizawa," Local Formulation of Interfacial Area Concentration in Two-Phase Flow." Int. J. Multiphase Flow', 12, 505-529 (1989)

7. Ishii M., Mishima K., Kataoka I., and Kocamustafaogullari G., "Two-fluid Model and Importance of the Interfacial Area in Two-Phase Flow Analysis," Proc. of the 9 th $U$, . National Congress of Applied Mechanics, Itheca, New York, pp. 73-80, June 21-25.

8. Ishii M., and Revankar S., "Local Interfacial Area Concentration Measurement in Bubbly Flow," Argonne National Laboratory Report,ANL-90/51 (1991)

9. Mishima K., and Ishii M., "Flow Regime Transition Criteria for upward Two-Phase Flow in Vertical Tubes." Int. J. Heat Mass Transfer, 27, 723-737 (1983)

10. Neal G.L., and Bankoif S.G., "A High Resolution Resistivity Probe for Determination of Local Void Properties in Gas-Liquid Flow." A.I.Ch.E.J., 9, 490494 (1963)

11. Park W.H., Kang W.K., Capes C.E., and Osberg, "The Properties of Bubbles in Fluidized Beds of Conducting Particles as Measured by an Electroresistivity Probe," Chem. Eng. Sci,24,856-865 (1969) 
12. Rigby G.R., van Blocakland G.P., Park W.H., and Capes C.E., " Properties of Bubbles in Three Phase Fluidized Beds as Measured by an Electroresistivity Probe," Chem. Eng. Sci., 25,1729-1714 (1970)

13. Hoffer M.S., and Resnick. W., " A Modified Electroresistivity Probe Techniques for Steady and Unsteady state Measurement in fine Dispersions. I. Hardware and Practical Aspects," Chem. Eng. Sci.,30, 473-480 (1975)

14. Burgess J.M., and Calderbank P.H., " Measurement of Bubble Parameters in TwoPhase Dispersion. - I. The Development of an Improvement Probe Technique," Chem. Eng. Sci.,30, 743-750 (1975)

15. Serizawa A., 1974 "Fluid Dynamic Characteristics of Two-Phase Flow." Ph.D. Thesis, Kyoto University, Japan

16. Veteau J.M.,"Contribution a'l'Etudes des Techniques de Mesure de l'Aire Interfaciale dans les Ecoulement d Bulles," ScD. Thesis, National Grenoble Polytechnique Institute, France, (1981)

17. Sekoguchi.. H. Fukui., Tsutsui M., and Nishikawa K., "Investigation into the Statistical Characteristics of Bubbles in Two-Phase Flow," Bull JSME 18, 379-404 (1975)

18. Buchholz R., J., Tsepetonides J., Steinemann J., and Onken U., "Influence of Gas Distribution on Interfacial Area and Mass Transfer in Bubble Column," Ger. Chem. Eng.,6,105-113 (1983)

19. Herringe R.A., and Davis, "Structure Development of Gas-Liquid Mixture Flows," Int. J. Fluid Mechanics,73, 97-123 (1976)

20. Lopez de Bertodano M., Lee, S.J., Lahey, R. T.Jr ., and Drew, D. A.,"The Predictions of Two-Phase Turbulence and Phase Distribution Phenomena Using a Reynolds Stress Model," J. Fluids Engng Des. 112, 107 (1990).

21. Sato, Y. and Sekoguchi, K. "Liquid velocity distribution in two-phase bubbly flow," Int. J. Multiphase Flow,2,79 (1975).

22. Serizawa, A., and Kataoka, I., "Turbulence suppression in bubbly two-phase flow," Nucl. Eng, and Design, 122, 1 (1990).

23. Serizawa, A., Kataoka,I., and Michiyoshi, I., "Turbulence structure of air-water bubbly flow -I. Measuring techniques," Int. J. Multiphase Flow, 2,221 (1974).

24. Wallis, G. B., " One-Dimensional Two-Phase Flow," McGraw-Hill, N.Y. 
25. Wallis, G. B., "The terminal speed of single drops or bubbles in an infinite medium," Int. J. Multiphase Flow, 1, 491, (1974)

26. Wang, S. K., Lee, S. J., Jones, O. C., Jr., and lahey, R. T., Jr., "Local Void Fraction Measuring Techniques in Two-Phase Flow Using Hot-Film Anemometry," ASMW Symposium Volume, G-000247.

27. Zuber, N., "On Dispersed Two-Phase Flow in Laminar Flow Regime," Chem. Eng. Sci.,19,897 (1, 4$)$.

23. Zuber, N., and Findley, J. A., "Average Volumetric Concentration in Two-Phase Flow systems," J. Heat Trans.. 87,453, (1965).

29 Drew D.R., and Lahey R.T., "Phase-Distribution Mechanisms in Turbulent Low Quality Twomphase Flow in a Circular Pipe"J. of Multiphuse Flow,117,91$106(1982)$

30. Lahey R.T.Jr.,"Turbulence and Phase Distribution Phenornena in Two-Phase Flow," Proc. 1987 ICHMT Seminar on Transient Multiphase Flow, Dubrobnik, Yugoslavia, 1987

31. Kataoka I., and Serizawa A., "Basic Equations of Turbulence in Gas-Liquid TwoPase Flow," J. of Multiphase Flow. Voi. 5, No.5, pp.843-855, 1989

32. Drew, D.A., and Lahey R.T.Jr., "The Virtual Mass and Lir Force on a Sphere Rotating and Straining Flow," J.Multiphase Flow, Vol.-13,No.1(1987) 


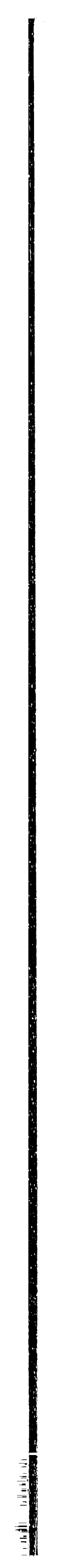

